\title{
APTAMERS THAT BIND FILOVIRUS GP: A NEW FRONTIER FOR DETECTION AND NEUTRALIZATION OF EBOLA AND MARBURG VIRUS
}

\author{
A Dissertation \\ presented to \\ the Faculty of the Graduate School \\ at the University of Missouri-Columbia \\ In Partial Fulfillment \\ of the Requirements for the Degree \\ Doctor of Philosophy \\ by \\ KWAKU DWUMAH TAWIAH \\ Dr. Donald H. Burke, Dissertation Supervisor \\ JULY 2020
}


The undersigned, appointed by the dean of the Graduate School, have examined the dissertation entitled

\title{
APTAMERS THAT BIND FILOVIRUS GP: A NEW FRONTIER FOR DETECTION AND NEUTRALIZATION OF EBOLA AND MARBURG VIRUS.
}

\author{
presented by Kwaku Dwumah Tawiah,
}

a candidate for the degree of Doctor of Philosophy,

and hereby certify that, in their opinion, it is worthy of acceptance.

Professor Donald H. Burke

Professor Thomas P. Quinn

Professor Antje Heese

Professor Mark Daniels

Professor Michael J. Petris 


\section{DEDICATION}

I dedicate this work to my parents, Mr. Jones S. Tawiah and Mrs. Alice Wiredu, my amazing siblings, and my beautiful wife.

Thank you all for the tremendous support and sacrifices. 


\section{Acknowledgement}

This work would not have been possible without immense support from the University of Missouri Biochemistry Department. They believed in me and offered me admission to their graduate school program. I am especially indebted to my advisor, Dr. Burke, who not only took me in as a graduate student but treated me like his son. Dr. Burke instilled in me many values and allowed me to grow as a scientist and as a person. I'd also like to thank my committee members for their guidance throughout my graduate school career.

My sincere gratitude to all the scientists in the Life Sciences building, especially the Virology Works in Progress group. Their constant feedback and relentless support, whenever the need arose, kept me going. A special thank you to past and present members of the Burke Lab. They provided a wonderful working environment that encouraged me to work hard and become the best version of myself. A very special thank you to Dr. Porciani, a research scientist in the Burke Lab, and Dr. Lange of Lange Lab. It has been an honor and joy working alongside and learning from such incredible and motivated scientists.

Finally, I will not be writing this dissertation if it weren't for my wonderful parents, Mr. J.S Tawiah and Ms. Alice Wiredu. They made no excuses in giving me and my siblings the best they had to offer even in the toughest moments. They made it their lives' goal to see us achieve above and beyond what they could ever dream of. I am grateful to God for blessing me with them. 


\section{TABLE OF CONTENTS}

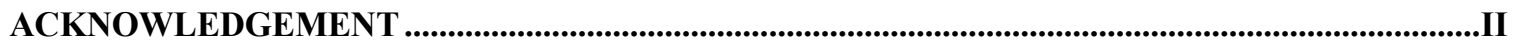

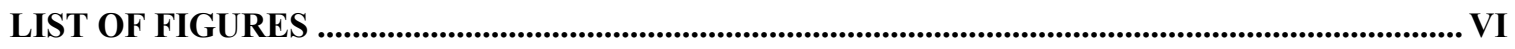

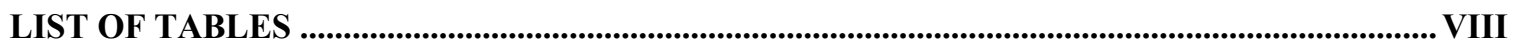

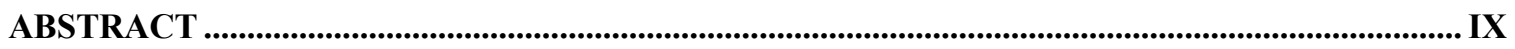

CHAPTER 1: BACKGROUND ON FILOVIRUSES AND FILOVIRUS DISEASE OUTBREAKS ...1

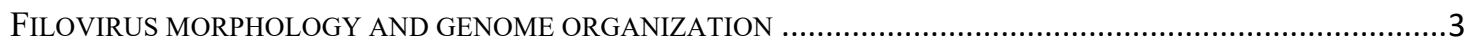

A CLOSER LOOK AT GP STRUCTURE AND GP-MEDIATED CELL ENTRY …..................................................

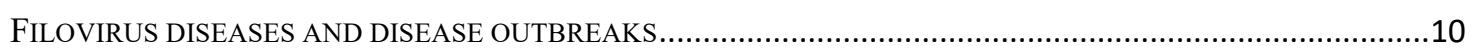

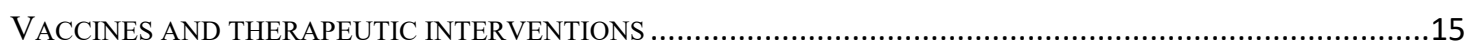

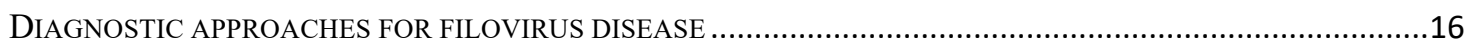

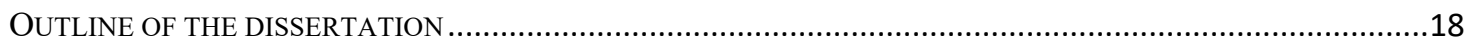

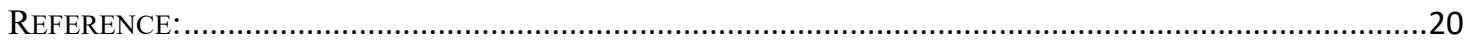

CHAPTER 2: TOWARD THE SELECTION OF CELL TARGETING APTAMERS WITH EXTENDED BIOLOGICAL FUNCTIONALITIES TO FACILITATE ENDOSOMAL ESCAPE OF

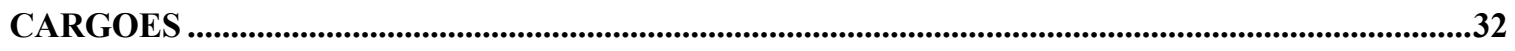

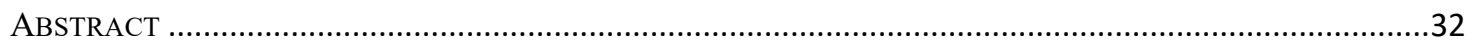

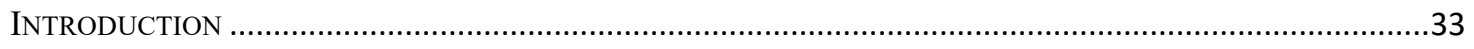

In Vitro SELECTION OF Cell TARgETING APTAMERS...........................................................................

RECENT ADVANCES IN CELL-INTERNALIZATION SELEX........................................................................

Strategies to Enhance AND Monitor Endosomal EscaPe OF CARGOeS .........................................53

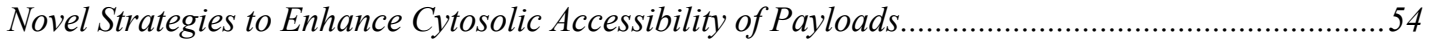

Traditional and Innovative Methods to Monitor Endosomal Escape of Cargoes .................................60

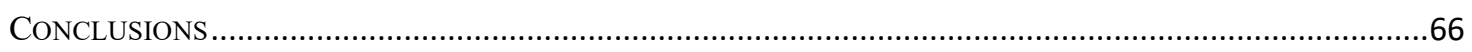

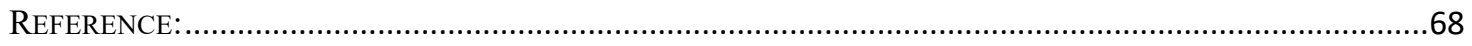

CHAPTER 3: IMPROVED VIRUS PURIFICATION AND SURFACE BIOTINYLATION OF

HIGHLY LYTIC ENVELOPED VIRUSES.................................................................................................81

ABSTRACT … 


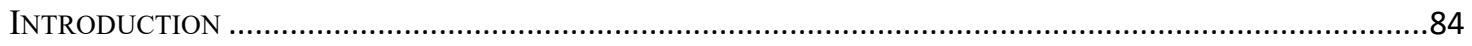

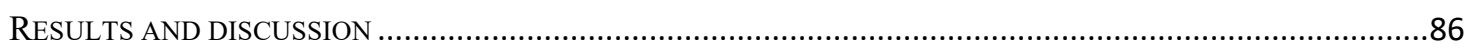

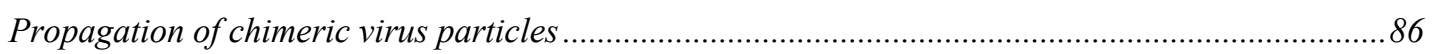

Three-step purification generates cleaner prep than two-step purification ........................................8

EXAMINING POTENTIAL CAUSE OF FRAGMENTED VIRUS PARTICLES IN THE VIRUS PREPS .........................93

END-POINT PCR RULES OUT CROSS-CONTAMINATION BETWEEN RVSV/EBOV_GP AND

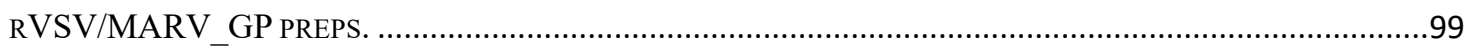

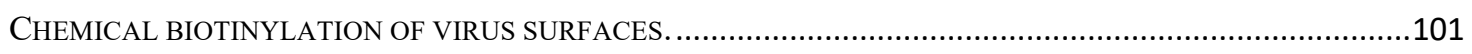

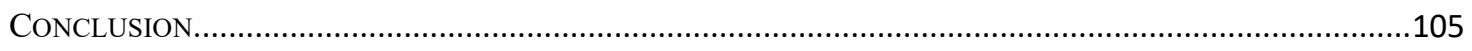

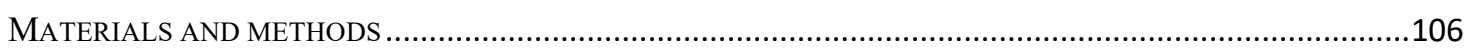

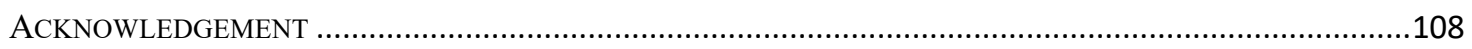

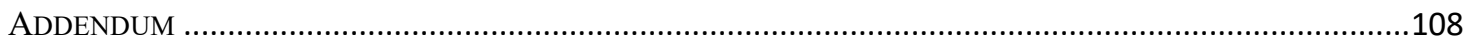

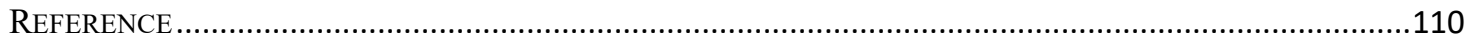

CHAPTER 4: DIFFERENTIAL RECOGNITION OF MARV GP AND EBOV GP BY AN SSDNA

APTAMER ..................................................................................................................................................................112

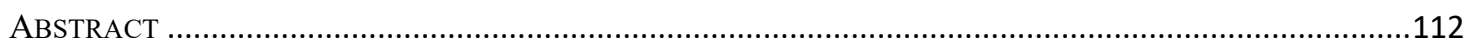

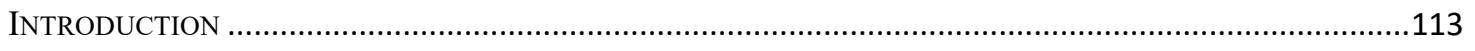

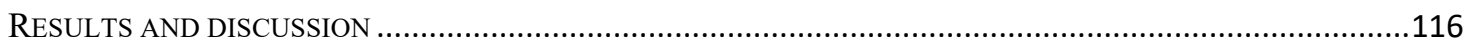

Selection of ssDNA aptamer against rVSV/MARV_GP viral particles. .......................................... 116

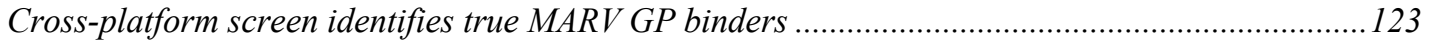

Binding and specificity of full-length and truncated aptamer variants............................................ 127

MH34 and MH36 do not compete with each other for binding to virus surface............................... 130

MH34 reliably detects MARV GP displayed on viruses when used in an ELONA. ......................... 132

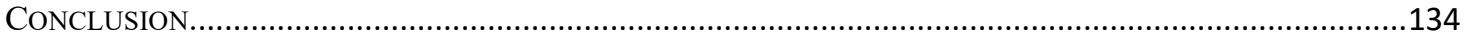

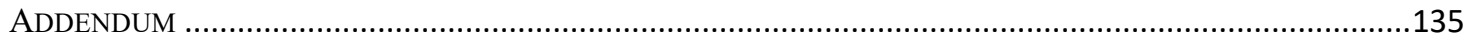

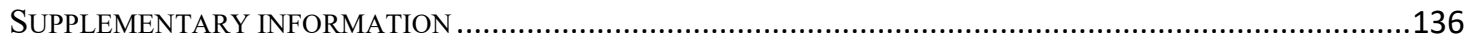

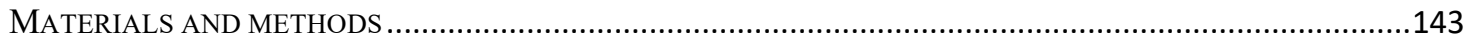

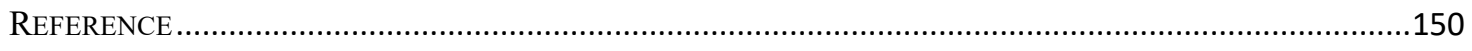




\section{CHAPTER 5: HYBRID SELEX GENERATES EBOLA VIRUS GLYCOPROTEIN TARGETING}

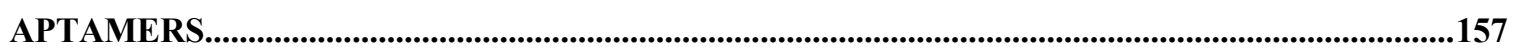

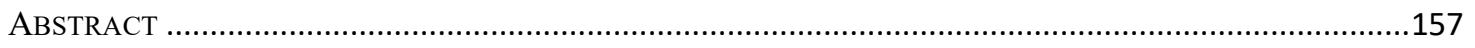

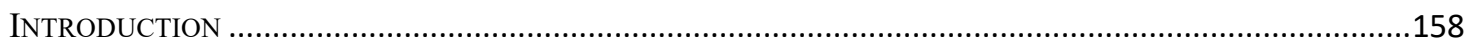

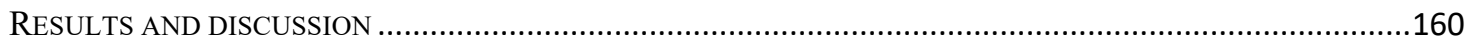

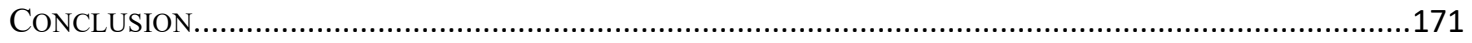

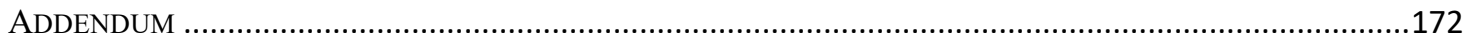

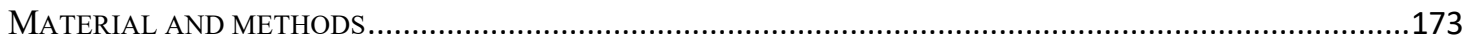

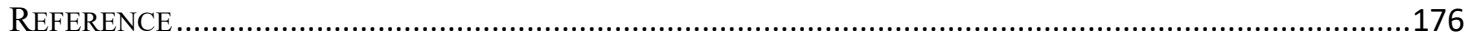

CHAPTER 6: PERSPECTIVE ON APTAMERS TARGETING VIRUS SURFACES: RAPID

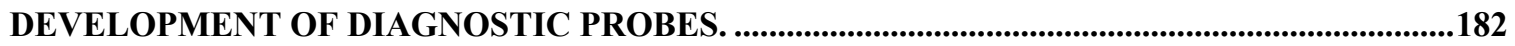

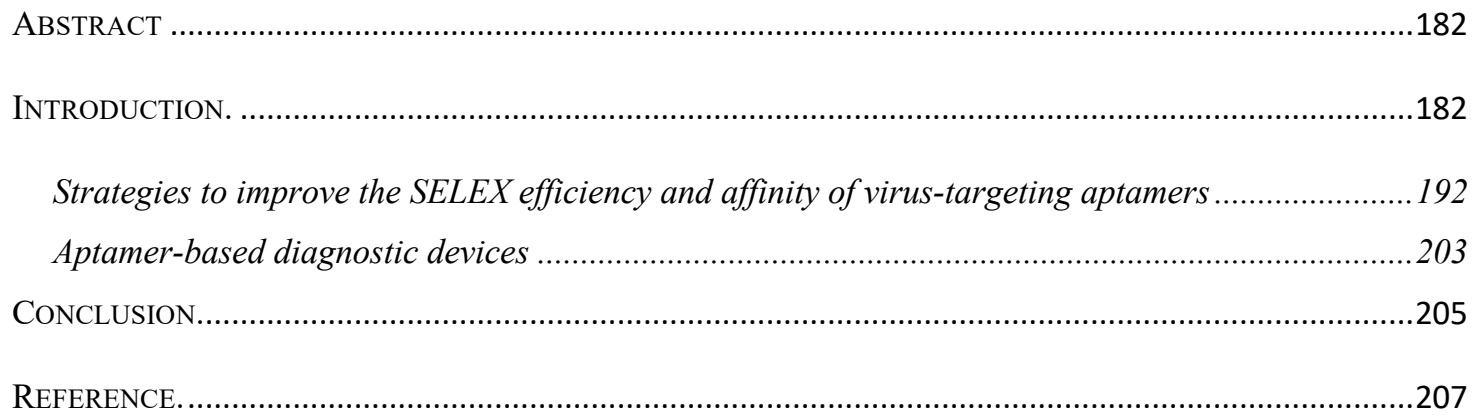

APPENDIX 1: THE EFFECTS OF NON-SPECIFIC NUCLEIC ACID BINDING ON THE EFFICIENCY OF COMPLEX TARGET SELEX..................................................................................216

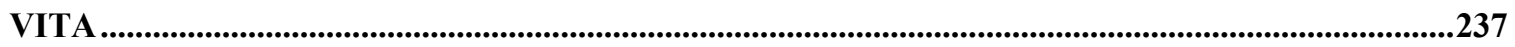




\section{LIST OF FIGURES}

FIGURE 1.1. OVERVIEW OF FILOVIRUS DIVERSITY AND CLASSIFICATION ................................... 2

FIGURE 2.1. RELATIONSHIPS AMONG THREE KEY FACTORS THAT GOVERN CYTOSOLIC DELIVERY: RECEPTOR EXPRESSION ON THE CELL SURFACE, RATE OF ENDOCYTOSIS AND ENDOSOMAL ESCAPE EFFICIENCY OF CARGOES.

FIGURE 2.2. SCHEMATIC ILLUSTRATION OF THE PROTEIN-BASED SELEX.................................. 43

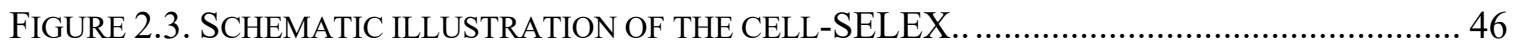

FIGURE 2.4. SCHEMATIC ILLUSTRATION OF THE CELL-INTERNALIZATION SELEX...................... 49

FIGURE 2.5. SCHEMATIC ILLUSTRATION OF THE EXPECTED VESICULAR TRAFFICKING OF INTERNALIZED APTAMERS AND POTENTIAL STRATEGY TO ENHANCE ENDOSOMAL ESCAPE. 58

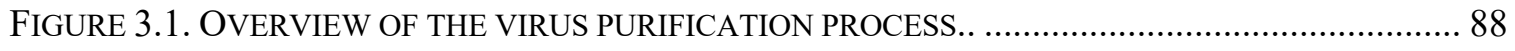

FIGURE 3.2. OVERVIEW AND EVALUATION OF THE TWO-STEP VIRUS PURIFICATION PROCESS. .... 90

FIGURE 3.3. IMAGE OF CLOUDY BAND AND EM IMAGES FROM 2-STEP AND 3-STEP PREPS ......... 92

FIGURE 3.4. NEGATIVE STAIN EM ANALYSIS OF VIRUS PREP FROM DIFFERENT ULTRACENTRIFUGATION TIMES 94

FIGURE 3.5. EM EVALUATION OF FRACTIONATED VIRUS BANDS FOLLOWING ULTRACENTRIFUGATION OF THE VIRUS SAMPLES THROUGH IODIXANOL GRADIENT . 95

FIGURE 3.6. NEGATIVE STAIN EM ANALYSIS OF VIRUS PREP FOLLOWING OVERNIGHT DIALYSIS 97

FIGURE 3.7. RNASE TREATMENT OF SAMPLE PREPS FAILED TO ELIMINATE RIBONUCLEOPROTEIN COMPLEX FROM THE VIRUS SAMPLES. 98

FIGURE 3.8. AGAROSE GEL EVALUATION OF ENDPOINT PCR THAT UTILIZED PRIMERS WITH SPECIFICITY FOR THE GENES FOR MARV GP AND EBOV GP.. 100

FIGURE 3.9. BIOTINYLATING SURFACE LYS ON VIRAL GP TO ENABLE CAPTURE BY STREPTAVIDINCOATED BEADS 102 
FIGURE 3.10. PLAQUE ASSAYS WERE USED TO EVALUATE WHETHER VIRUS SURFACE-DISPLAYED BIOTIN WAS SUFFICIENT FOR FACILITATING IMMOBILIZATION ON THE SURFACE OF SACOATED BEADS 104

FIGURE 4.1. OVERVIEW OF THE VIRUS PROPAGATION, PURIFICATION AND EVALUATION PROCESS.. 118

FIGURE 4.2. OVERVIEW OF THE SELECTION STRATEGY. 120

FIGURE 4.3. BINDING SPECIFICITY OF SSDNA APTAMER LIBRARY SELECTED FOR BINDING TO MARV GP 122

FIGURE 4.4. POST-SELEX, CROSS-PLATFORM SCREENING APPROACH . 126

FIGURE 4.5. MH34 BINDING AND BINDING SPECIFICITY WERE ASSESSED USING MULTIPLE BINDING ASSAYS

FIGURE 4.6. COMPETITION ASSAY SHOWS THAT MH34 AND MH36 BIND TO DIFFERENT EPITOPES OF MARV GP. 131

FIGURE 4.7. SCHEME AND EXPERIMENTAL DETECTION OF RVSV MARV GP USING ANTIBODYAPTAMER BASED ELONA. 133

FigURE 5.1. OVERVIEW OF THE CELL-SELEX PHASE OF THE HYBRID SELEX PROCESS.. 162

FIGURE 5.2. FLOW CYTOMETRY WAS USED TO ASSESS BINDING OF THE ROUNDS 5 AND 6 POPULATIONS (SELECTED TO BIND HELA CELLS TRANSFECTED WITH EBOV GP 164

FIGURE 5.3. OVERVIEW OF THE BEAD-BASED VIRUS CAPTURE AND SELEX PROCESS.. 166

FIGURE 5.4. FLOW CYTOMETRY WAS USED TO ASSESS BINDING OF THE ROUNDS (6 ROUNDS ON EBOV GP-EXPRESSING HELA CELLS, 1 ROUND ON BEAD-CAPTURED RVSV/EBOV_GP).

FIGURE 5.5. COMPREHENSIVE ENRICHMENT ANALYSIS OF THE EVOLVED LIBRARY SELECTED FOR BINDING TO EBOV GP. 170

FIGURE 6.1. OVERVIEW OF THE CONVENTIONAL APTAMER SELECTION PROCESS. 194

FIGURE 6.2. OVERVIEW OF A RAPID VIRUS-SELEX PLATFORM. 206 


\section{LIST OF TABLES}

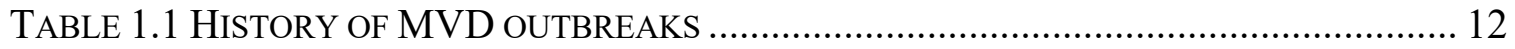

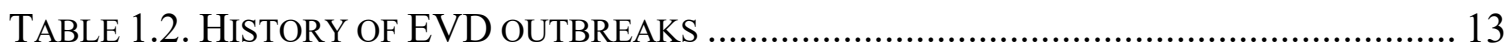

TABLE 2.1. APTAMERS THAT BIND CELL-SURFACE MARKERS SELECTED BY PROTEIN-

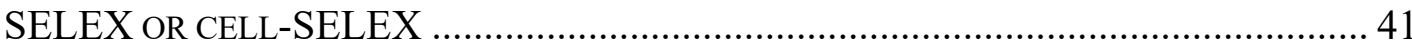

TABLE S4.1. SELECTION CONDITIONS FOR MARV GP SELECTION ................................. 141

TABLE S4.2. SANGER SEQUENCING RESULTS FOR MH CLONES. ................................... 142

TABLE A7.1. SUMMARY OF SELECTION CONDITIONS FOR FILOVIRUS SELEX ATTEMPT 1220

TABLE A1 7.2. SUMMARY OF SELECTION CONDITIONS F FILOVIRUS SELEX ATTEMPT 1 . 225

TABLE A 7.3. SANGER SEQUENCING DATA FROM RND 8.1 POPULATION. .......................... 229

TABLE A 7.4. SANGER SEQUENCING DATA FROM RND 12.1 POPULATION. ........................ 230 


\begin{abstract}
Aptamers are single chained, nucleic acid-based affinity probes that bind to their targets with strong affinity and specificity. They are made through an in vitro combinatorial selection method, wherein large libraries of nucleic acids with randomized sequences are subjected to an iterative process of affinity enrichment, partitioning, and amplification. Evolved libraries are sequenced, and the individual sequences are screened and characterized for their structure and function. Aptamers have been developed to target many molecules, including small molecules, purified proteins, whole cells, bacteria, and viruses. They have been developed for therapeutics and as research and diagnostic probes. Aptamers that have an affinity for virus surfaces are excellent probes for developing low-cost biosensors and potentially antiviral therapeutics.
\end{abstract}

In this work, I present the development of aptamers that have an affinity for filovirus surfaces. I first describe the development of an improved method for purifying highly lytic vesicular stomatitis virus-based filovirus GP displayed surrogate viruses. Filoviruses are highly pathogenic and thus require highly secured containment facilities for their studies. The use of attenuated surrogates facilitates filovirus research at biosafety level 2 facilities. This work outlines the steps required to propagate and generate pure virus particles to be used as selection targets. I then describe the development of aptamer probes that differentially recognize GPs from MARV and EBOV. This work represents the first step in the development of aptamer based-low-cost point of care devices for filovirus disease diagnostics. Finally, this work describes the use of a hybrid selection 
approach that combines two different selection platforms to generate aptamers that bind to EBOV surfaces 


\section{Chapter 1: Background on filoviruses and filovirus}

\section{disease outbreaks}

Filoviruses are pathogenic negative-sense enveloped RNA viruses that infect humans, non-human primates, bats, and other mammals. Some filoviruses cause severe hemorrhagic fevers in humans and non-human primates ${ }^{1}$. Previous outbreaks of Ebola virus disease (EVD) and Marburg virus disease (MVD) have had high case mortality rates $(40 \%-90 \%)^{2,3}$. Filoviruses belong to the Filoviridae family, which has three different genera: Ebolavirus, Marburgvirus, and Cuevavirus (Fig 1.1). The Ebolavirus genus has classified species: Ebola virus (EBOV), Sudan virus (SUDV), Bundibugyo virus (BDBV), Reston virus (RESTV), and Tai Forest virus (TAFV). All the species in the Ebolavirus genus but RESTV, cause EVD. Marburgvirus has two known species, Marburg virus (MARV) and Ravn virus (RAVV), both of which are linked to lethal MVD in humans and non-human primates. Finally, the Llovio virus (LLOV) is the only known species under Cuevavirus. There is no evidence of LLOV pathogenicity in primates but it is linked to a lethal disease in bats ${ }^{4,5}$. 


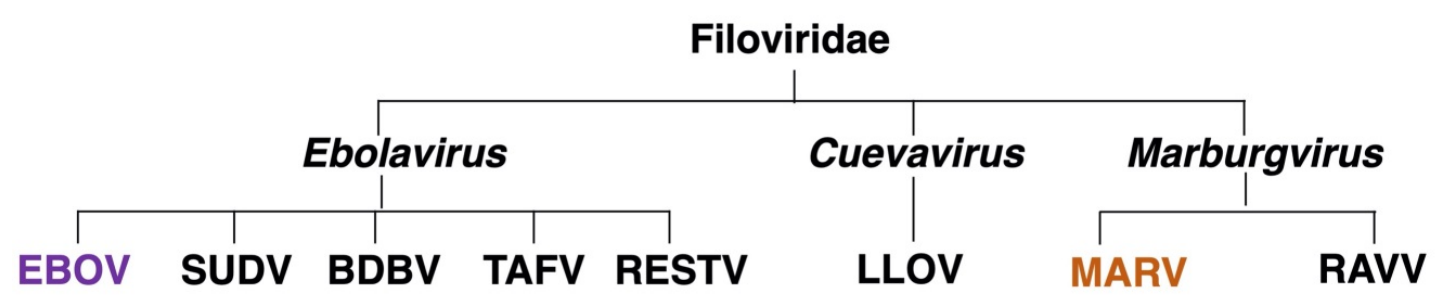

Figure 1.1. Overview of filovirus diversity and classification. The work presented in this dissertation focuses on the glycoproteins (GP) from EBOV (purple) and MARV (orange). GP amino acid sequences differ by approximately $30 \%$ between EBOV and MARV 


\section{Filovirus morphology and genome organization}

Filovirus particles differ in their length and shape. At peak infection, virion particle length ranges from $790 \mathrm{~nm}$ for MARV to $970 \mathrm{~nm}$ for EBOV. Both MARV and EBOV species have the same diameter and density $(80 \mathrm{~nm}$ and $1.14 \mathrm{~g} / \mathrm{ml})$. Electron micrographs show elongated filamentous (occasionally branched) virion forms $(\text { Fig 1.2 })^{6,7}$. Filoviruses maintain a 19 kilobase genome that encodes 7 distinct structural proteins. They are nucleoprotein (NP), viral protein (VP) 24, VP30, VP35, and VP40, an RNA-dependent RNA polymerase (RdRP) (L), and a glycoprotein (GP). Unlike other non-segmented negative-stranded RNA viruses, the genes encoded in the filovirus genome have their respective open reading frames (ORF). The genes overlap or remain separated by short non-conserved nucleotides except the region between GP and VP30, which is relatively larger. Non-coding cis-acting elements flank the genome ends and are suspected to impact replication, transcription initiation, and viral genome encapsulation and packaging 8,9 .

While several studies have brought insights into the functions of filoviral proteins, there are still a lot learn about them. Current literature suggests that in filoviruses, VP40 likely functions as a matrix protein (M) due to its hydrophobic profile and high abundance on the inner part of the virion membrane. It has also been shown to be sufficient for virus budding. Expression of VP40 in mammalian cells without the other filovirus proteins results in a robust formation and egress of filovirus-like particles ${ }^{10}$. Crystallographic studies have revealed that VP40 lacks a transmembrane domain but strongly binds to the interior surface of the plasma membrane through the $\mathrm{C}$-terminal domain. The $\mathrm{N}$-terminus of VP40 interact to form dimers in the cytoplasm. When VP40 is localized at the plasma 
membrane, it reorganizes to form hexamers that are essential for virus budding ${ }^{11}$. There are several biochemical and biophysical evidence that suggest that VP40 is capable of penetrating into the lipid bilayer and initiate membrane curvature leading to virus budding ${ }^{12}$.VP24 is membrane-associated and sometimes described as a secondary matrix protein, although its function is unclear. In EVD, VP24 is suspected to contribute to the ability of EBOV to evade antiviral effects of interferons (IFN). Mammalian cell assays in-vitro show VP24 binds the nuclear localization signal of STAT1 and blocks its nuclear accumulation, which is a key step in the IFN signaling. VP35 has also been linked to host immune evasion. In vitro work done in mammalian cells show that both EBOV and MARV VP35 antagonize IFN by inhibiting IFN regulatory factor 3 (IRF3) and IRF7 ${ }^{13}$. Cocrystal structures of the RNA binding domain of VP35 from MARV and dsRNA suggests that VP35 fully coats the dsRNA during virus replication, thus interfering with pattern recognition mechanisms utilized by dsRNA sensing receptors such as RIG-I ${ }^{14}$. VP30 is considered as a minor NP. Both NP and VP30 interact strongly with RNA and form the ribonucleoprotein complex, together with the L, and VP35 proteins. In a phosphorylated state, VP30 supports viral transcription (mRNA synthesis) in EBOV. The L protein is the enzymatic component of the RdRP complex with VP35 as a cofactor. NP is the principal structural protein of filoviruses and is involved in virus replication in EBOV. Biochemical and electron microscopy studies revealed that the N-terminus of NP of EBOV is important for NP-NP interactions that could serve as scaffold for RNP assembly ${ }^{15}$. 
Finally, GP is the only protein displayed on the surface of the virus and is responsible for receptor binding, fusion, virus entry, and tropism ${ }^{6,16,17}$. There is a distinction between EBOV and MARV GP gene organization and transcription. The MARV GP gene encodes for a single GP product. In contrast, the EBOV GP gene codes for additional proteins due to polymerase slippage during transcription by the virus. Slippage of the $\mathrm{L}$ polymerase results in the incorporation of non-template encoded adenosines into the growing mRNA chain resulting in novel ORFs. The unperturbed transcript generates a non-structural secreted glycoprotein (sGP) that is secreted from infected cells $(80 \%$ on the time). Approximately $20 \%$ of the time, the insertion of Adenosine occurs and cause a frameshift that results in a membrane displayed GP (GP). Sometimes the slippage causes a deletion of an adenosine or addition of two adenosines, which results in the production of a second secreted protein (ssGP). It is not entirely clear how much of ssGP is produced. Although the function of sGP is not clear, it is linked to host immune modulation since it acts as a decoy molecule and anti-inflammatory factor ${ }^{9,18}$. Filovirussurface displayed GP has emerged as a major target for therapeutic and diagnostic developments. GP remains the only known target for both neutralizing and nonneutralizing antibodies during filovirus infection. The work presented in this dissertation describes the development of nucleic acid-based probes that have affinity for GPs from EBOV and MARV. These probes may block filoviral infections or serve as excellent affinity probes for developing simple, low-cost biosensors for viruses. 


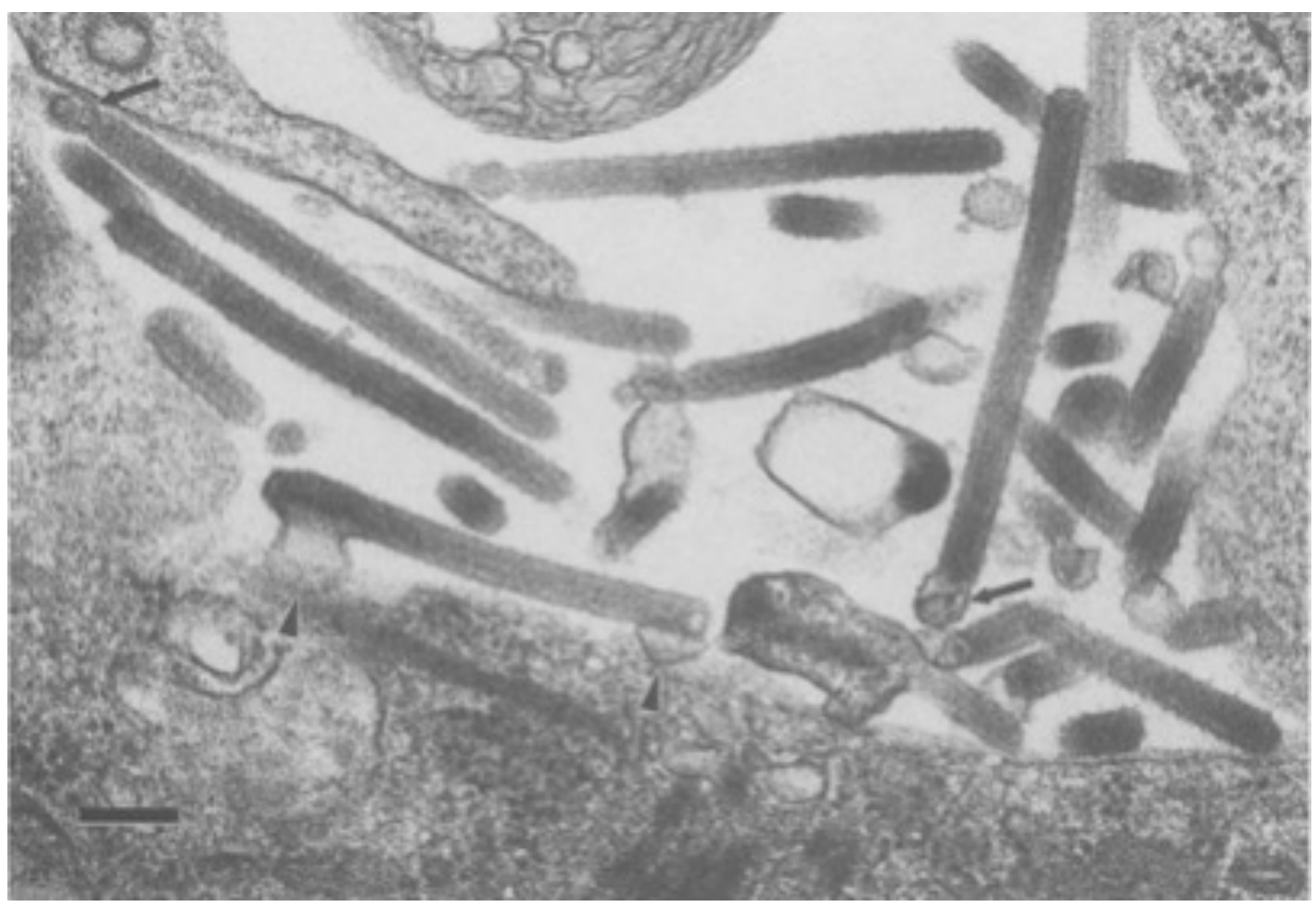

Figure 1.2. Thin-section transmission electron micrographs of EBOV particles showing long filamentous particles. (Permission to reuse from Geisbert, T. W7 license number 4840840841338) 


\section{A closer look at GP structure and GP-mediated cell entry}

During viral assembly, a host furin protease in the ER cleaves the full-length GP (676 residues, also known as GP0) to generate two subunits (GP1 \& GP2) connected through disulfide bonds ${ }^{19}$. GP is a trimer of these heterodimers. Three GP1-GP2 heterodimers form the chalice-shaped spike (450kDa) on the virus surface. The C-terminus of GP1 has a heavily glycosylated mucin-like domain and a glycan cap beneath it (Fig 1.3). The receptor-binding site (RBS) is buried underneath the glycan cap. The furin cleavage exposes an N-terminal fusion peptide on the GP2 unit. GP2 is a trimeric membrane unit of GP that hosts all the critical architecture for membrane fusion. The fusion loop is held in a compact conformation by two heptad repeats (HR1 \& HR2), a membrane-proximal external region (MPER), and the transmembrane domain located at the C-terminus.

We developed platforms that utilized HeLa cells that display filovirus GP as targets for a combinatorial selection process and as screening platform for filovirus GP binding aptamers. To the best of our knowledge, this the first report of selections to generate aptamers that have affinity for virus proteins displayed on mammalian cell surfaces. Filoviruses acquire GPs from membranes of infected cells during the assembly of the nascent virions. The platform developed in this work, exploits the phenomena that filovirus GPs are acquired from host cell displayed virus GP during the infection process. Both selection and screening approaches are inspired by extensive research and development on aptamers selected to bind cell surfaces. Chapter two reviews some of the approaches used to develop cell surface aptamers and molecular applications of such aptamers. 
The detailed mechanism of the filovirus infection and the life cycle are under investigation. Filoviruses use infection mechanisms that maximize infection while reducing immune detection. The virus GP mediates host cell recognition, attachment, and uptake by binding to host attachment factors. Several cellular attachment factors have been reported. For example, the carbohydrate recognition domains (CRD) in c-type lectins and other cell-specific lectins interact with the glycans on the GP surface. The Tcell immunoglobulin mucin domain (TIM), the Tyro3 protein kinase, and folate receptora (FR-a) are all linked to the initial attachment of virions to host cells ${ }^{20-23}$. Virions enter cellular endosomes through macropinocytosis and are eventually cleaved by endosomal cathepsins to expose the RBS. RBS binds to the domain $\mathrm{C}$ on the endosomal cholesterol transporter, NPC1-C and triggers membrane fusion that ejects the virus genome into the cytoplasm of the infected host cell ${ }^{8,24}$. 
a
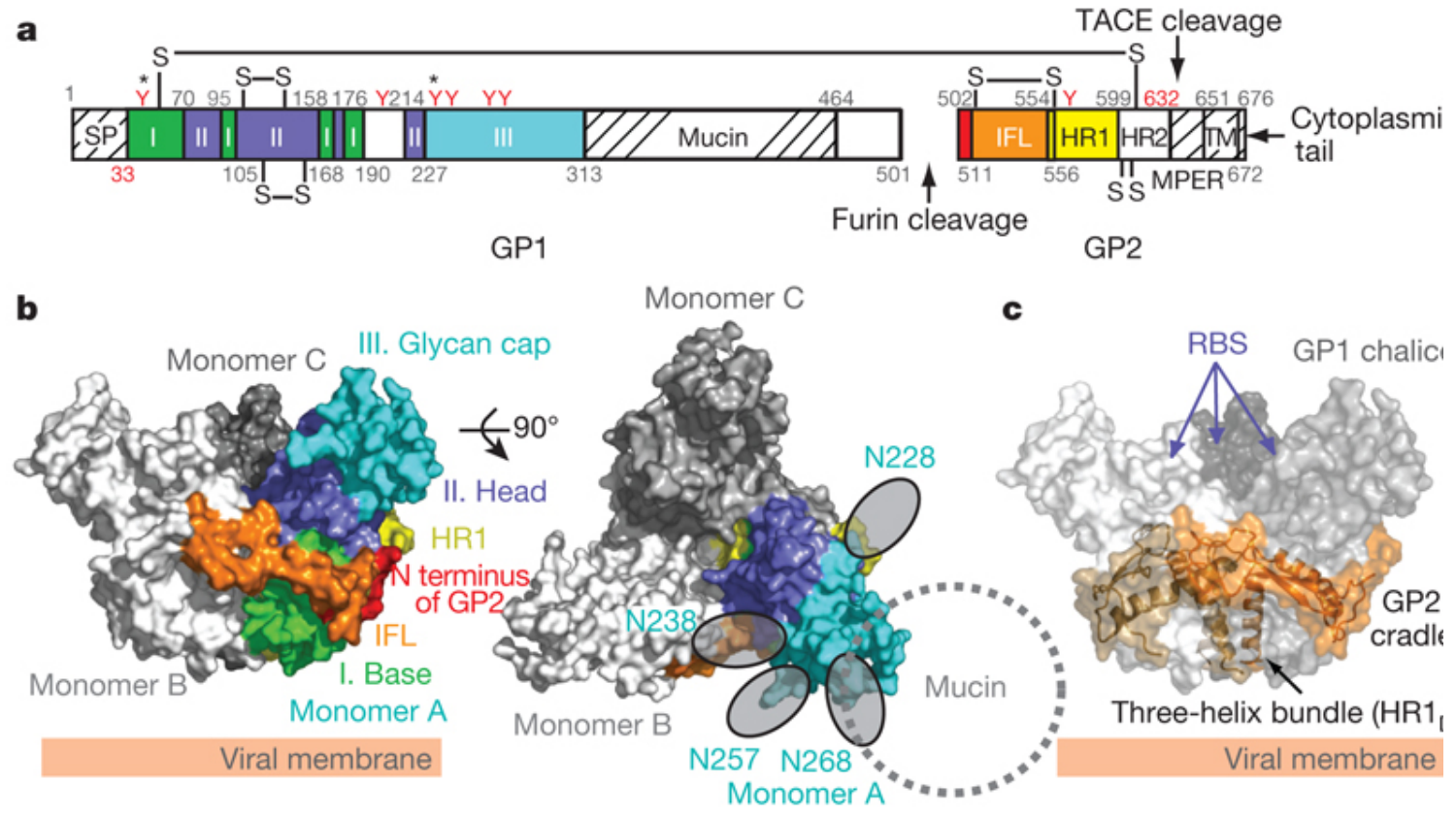

Figure 1.3. Structure of EBOV GP. A. Schematic of the domains of GP as observed in crystal structure. B. A view from the side (left) and from the top (right) of the molecular surface of the GP trimer. The monomer is colored according to the scheme in A. C. Molecular surface of the EBOV GP chalice and cradle. The three GP1 subunits that form the chalice are shown in various shades of grey and GP2 subunits forming the cradle are shown as ribbons, in various shades of orange, underneath the transparent molecular surface. The putative receptor-binding sites (RBS) are recessed in the inner bowl of the GP trimer. (permission to reuse from Lee, J. E et $\left.a l^{25}\right)$. 


\section{Filovirus diseases and disease outbreaks}

Immunohistochemistry analysis of tissues from filovirus disease patients suggests that filoviruses have broad cellular tropism that includes monocytes, hepatocytes, adrenal gland tissue, epithelial, and endothelial cells $\mathrm{s}^{6,26,27}$. However, for initial infections, filoviruses preferentially infect and replicate in macrophages and dendritic cells ${ }^{22}$. Human transmissions occur through direct contact with infected bodily fluids. Also, cases of sexual transmission by survivors have been reported in the past, suggesting virion presence in immune-privileged sites ${ }^{28-30}$. The virus incubation period is about 4-16 days post-infection. The onset of diseases is associated with fever, headache, malaise, myalgia, and conjunctivitis. Severe infections cause swelling and bleeding in several organs and tissues. Additionally, virus replication in vivo triggers the release of pro-inflammatory cytokines, chemokines, reactive oxygen species, and nitric oxide. The massive release of these molecules disrupts many host immune modulation pathways. Virus replicationmediated inhibition of host antiviral responses of host's innate immune response is partly responsible for the severe lethality of filoviruses ${ }^{1,31-35}$. Without therapeutic intervention, death typically occurs 5-6 days after the onset of symptoms ${ }^{6,36}$.

The first recorded filovirus disease outbreaks was an MVD outbreak in 1967 that happened in Marburg and Frankfurt, Germany and in Belgrade, Serbia ${ }^{37}$. There have been several filovirus diseases outbreaks since then, including thirteen outbreaks of MVD and thirty-seven outbreaks of EVD (Table 1.1 and Table 1.2). Distinctly, the 2013-2016 outbreak of EVD in West Africa was the largest outbreak of EVD or filoviruses ever recorded. This outbreak had more cases and deaths than all the previous outbreaks 
combined (28,616 cases, 11,310 case fatalities). The most recent outbreak of EVD happened in 2018 in the Democratic Republic of Congo (3,805 cases, 2,268 case

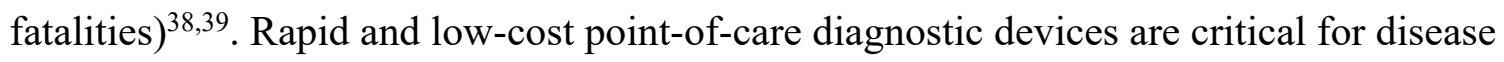
management and surveillance of filoviruses during outbreaks, especially in resource-poor communities. A major focus of this dissertation is the development of nucleic acid-based probes that have an affinity for MARV or EBOV surfaces. Part of the work presented in this dissertation is the initial step towards the development of low-cost point of care devices and assays that do not require complex instruments such as thermocyclers and reagents that require a cold chain. 


\section{Table 1.1. History of MVD outbreaks}

\begin{tabular}{|c|c|c|c|c|}
\hline Year & Location & Subtype & Total cases & Case mortality \\
\hline 1967 & Germany \& Serbia & MARV & 31 & 7 \\
\hline 1975 & South Africa & MARV & 3 & 1 \\
\hline 1980 & Kenya & MARV & 2 & 1 \\
\hline 1987 & Kenya & RAVV & 1 & 1 \\
\hline 1990 & Russia & MARV & 1 & 1 \\
\hline $1998-2000$ & D.R. Congo & MARV \& RAVV & 153 & 128 \\
\hline $2004-2005$ & Angola & MARV & 252 & 227 \\
\hline 2007 & Uganda & MARV \& RAVV & 3 & 1 \\
\hline 2008 & United States & MARV & 1 & 0 \\
\hline 2008 & Netherlands & MARV & 1 & 1 \\
\hline 2012 & Uganda & MARV & 15 & 4 \\
\hline 2014 & Uganda & MARV & 1 & 1 \\
\hline 2017 & Uganda & MARV & 3 & 3 \\
\hline Total & & & 467 & 376 \\
\hline
\end{tabular}


Table 1.2. History of EVD outbreaks

\begin{tabular}{|c|c|c|c|c|}
\hline Year & Location & Subtype & Total cases & Case mortality \\
\hline 1976 & Sudan & SUDV & 284 & 151 \\
\hline 1976 & D.R. Congo & EBOV & 318 & 280 \\
\hline 1976 & United Kingdom & EBOV & 1 & 0 \\
\hline 1977 & D.R. Congo & SUDV & 1 & 1 \\
\hline 1979 & Sudan & EBOV & 34 & 22 \\
\hline \multirow[t]{2}{*}{ 1989-1990 } & Philippines \& & RESTV & 4 & 0 \\
\hline & United States & & & \\
\hline 1994 & Gabon & EBOV & 52 & 31 \\
\hline 1994 & Ivory Coast & TAFV & 1 & 0 \\
\hline 1995 & D.R. Congo & EBOV & 315 & 250 \\
\hline 1996 & Gabon & EBOV & 37 & 21 \\
\hline 996-1997 & Gabon & EBOV & 60 & 45 \\
\hline 1996 & South Africa & EBOV & 2 & 1 \\
\hline 1996 & Russia & EBOV & 1 & 1 \\
\hline $2000-2001$ & Uganda & SUDV & 425 & 225 \\
\hline \multirow[t]{2}{*}{ 2001-2002 } & Gabon \& & EBOV & 123 & 97 \\
\hline & Congo-Brazzaville & & & \\
\hline $2002-2003$ & Congo-Brazzaville & EBOV & 143 & 128 \\
\hline 2003 & Congo-Brazzaville & EBOV & 35 & 29 \\
\hline 2004 & Sudan & SUDV & 17 & 7 \\
\hline 2004 & Russia & EBOV & 1 & 1 \\
\hline 2005 & Congo-Brazzaville & EBOV & 12 & 10 \\
\hline 2007 & D.R. Congo & EBOV & 264 & 186 \\
\hline 2007-2008 & Uganda & BDBV & 131 & 42 \\
\hline
\end{tabular}




$\begin{array}{lllll}2008 & \text { Philippines } & \text { RESTV } & 6 & 0 \\ 2008-2009 & \text { D.R Congo } & \text { EBOV } & 32 & 15 \\ 2011 & \text { Uganda } & \text { SUDV } & 1 & 1 \\ 2012 & \text { Uganda } & \text { SUDV } & 11 & 4 \\ 2012 & \text { D.R. Congo } & \text { BDBV } & 36 & 13 \\ 2012-2013 & \text { Uganda } & \text { SUDV } & 6 & 3 \\ 2013-2016 & \text { Multiple countries } & \text { EBOV } & 28,616 & 11,310 \\ 2014 & \text { D.R. Congo } & \text { EBOV } & 69 & 49 \\ 2017 & \text { D.R. Congo } & \text { EBOV } & 8 & 4 \\ 2018 & \text { D.R. Congo } & \text { EBOV } & 54 & 33 \\ 2018-2019 & \text { D.R. Congo } & \text { EBOV } & 1,604 & 1,074 \\ \text { Total } & & & 32,703 * & 4,034 * \\ \text { Total } & & & & 2,724\end{array}$

Include 2013-2016 outbreak * 


\section{Vaccines and therapeutic interventions}

The EVD outbreak in 2013 sounded the alarm to the world about the need for effective therapeutic intervention. Initial results on the efficacy of an rVSV-vectored vaccine on preventing EVD showed great promise back in $2017^{40-42}$. Recently, the United States Food and Drug Administration (FDA) approved this vaccine. It prevents Zaire Ebolavirus-caused EVD but is not effective in preventing disease caused by other species of Ebolavirus or by MARV $\mathrm{M}^{43-45}$.

Monoclonal antibody (mAb)-based drugs, including REGN-EB3, MAb114, and ZMAPP, are in various developmental phases. The FDA approved REGN-EB3 and MAb114 for emergency use during the recent outbreak because they were efficacious in a pilot study. REGN-EB constitutes a three-antibody cocktail (REGN3471, 3470, and 3479) that targets non-overlapping epitopes on the EBOV GP. REGN3471 binds within chalice structure, and is also suspected to make contact with the glycan cap, REGN3470 was shown to bind parallel to the viral surface on the outside of the glycan cap. Finally, REGN3479 binds to the base of GP between the interface of GP1 and GP2 ${ }^{46}$. Conformational-epitope mapping assays show that Mab114 interacts with the glycan cap and binds close to the receptor-binding domain of the GP. Notably, mAb100 remains bound to EBOV GP following proteolytic cleavage. Binding was shown to prevent NPC1-C binding. Both drugs are delivered in a single dose via intravenous infusions ${ }^{47-49}$. The exact mode of action utilized by these antibodies to reduce virus loads is not entirely clear. However, like most Abs, they could directly neutralize EBOV or trigger the host innate immune cells through the Fc receptors ${ }^{50}$. Both the glycan cap and MLD are 
removed by cathepsin cleavage, therefore it is less likely for Abs that bind to the glycan cap or MLD to block virus entry. EBOV GP has a metastable pre-fusion conformation that changes spontaneously to into a post-fusion conformation. Abs that bind to the base of GP are suspected to stabilize the pre fusion conformation, which prevents the conformational changes required for entry. Finally, Gilead developed a promising broadspectrum small molecule nucleotide analogue prodrug called (Remdesivir). Both in vitro and in vivo work from many groups have validated the antiviral effects of Remdesivir on several enveloped RNA viruses, including filoviruses and coronaviruses. Remdesivir facilitated a delayed chain termination for the nascent RNA during virus transcription. Remdesevir gets incorporated into the nascent in place of the natural adenosine triphosphate (ATP). Incorporation of the metabolized form of Remdesivir causes premature termination after three to four more nucleotides are added to the RNA chain. In the pilot study, Remdesivir reduced case mortality by half. While promising, it was comparatively less effective than the two antibody drugs ${ }^{51}$. Currently, there are no FDA approved drugs or vaccines for MVD and the rVSV-EBOV vaccine, and the antibodybased drugs (REGN-EB3 and mAb114) do not work for MVD. Supportive care such as rehydration and the treatment of patient-specific symptoms remain the main interventions ${ }^{3,39}$.

\section{Diagnostic approaches for filovirus disease}

Differentiating between EVD and MVD based on clinical symptoms can be challenging due to the similarities in the clinical signs and symptoms presented by patients early in the course of infection. Also, it is difficult to distinguish filoviral diseases from other 
infectious diseases that are common in sub-Saharan Africa, including malaria, typhoid fever, and yellow fever. As such, accurate and timely diagnoses are essential for the efficient management of filovirus diseases. Moreover, Filovirus diseases have a better prognosis when detected early. For example, the approved antibody drugs (REGN-EB3 and mAb114) had better therapeutic outcomes on patients at the early stage, compared to those at the later stage of the disease ${ }^{51}$. Also, ongoing research suggests that the current vaccine can be used as post-exposure prophylaxis. EBOV exposed patients (45 patients), who received the rVSV-EBOV vaccine produced neutralizing antibodies and none of them developed infection ${ }^{26,52,53}$. However, there are several limitations to this study including the lack of randomized control and an inability to ascertain a definite exposure to the EBOV. EVD and MVD diagnosis during outbreaks relies heavily on polymerase chain reaction (PCR)-based tests, although there are also other classical viral detection methods such as Enzyme-Linked Immunosorbent Assay (ELISA), antigen detection tests, serum neutralization tests, and viral cultures ${ }^{54,55}$.

While PCR-based tests are sensitive, they are logistically challenging when outbreaks occur in rural and inaccessible areas. Serological assays such as ELISA take time to develop during novel virus outbreaks, are less sensitive, and likewise face logistical challenges when outbreaks occur in resource-limited settings ${ }^{2,56-60}$. There is a need for new diagnostic technologies that are simple and easy to operate, low-cost, and readily deployable to resource-poor settings. In chapters 4 and 5, I developed aptamer probes that have affinity for MARV and EBOV surfaces. The chemical properties of these aptamer 
probes make them excellent reagents for developing low-cost EBOV and MARV biosensors.

\section{Outline of the dissertation}

Chapter 2 introduces nucleic-acid-based probes known as aptamers as excellent affinity probes for targeting cell surfaces. Aptamers that target cell surfaces can be utilized for targeted delivery in diseased cells and biomarker discovery probes for diagnostic purposes. Also, the interaction between aptamer and cell surface receptors can be exploited to influence the biology of the receptors. Aptamer binding can antagonize or enhance these surface receptors ${ }^{61,62}$. This review focuses on cancer cell surfaces. However, the principles underlining cell SELEX applies to cell displayed viral proteins and whole viruses as well. This review has been published in Biomedicine and was coauthored with Dr. David Porciani under the supervision of Dr. Donald Burke.

Chapter 3 covers a method development for the purification and surface biotinylation of highly lytic enveloped viruses. I detailed a three-step process used to purify chimeric vesicular stomatitis virus particles that display filovirus glycoprotein. The purification processes described here were utilized to generate virus particles that served as targets for the works described in chapters 4 and 5. This work was done with the assistance of Amanda Paz and Dr. David Porciani under the supervision of Dr. Donald Burke. This work will be adapted for submission as a method development paper. 
Chapter 4 describes the generation of ssDNA aptamers that bind to MARV surface GP. I developed a selection technology platform that utilizes virus particles as selection targets. Using this platform, I identified two aptamers that bind to MARV GP displayed on the surface of viruses and on cells. The two aptamers did not compete with each other to access the virus surface. Finally, I demonstrate that the aptamer can be used to detect the target virus particles. This work was done with the assistance of Amanda Paz, Dr. David Porciani, Dr. Carolina Camargo, and Dr. Margaret J. Lange under the supervision of Dr. Donald Burke. The work will be submitted as a manuscript for publication.

Chapter 5 reports a hybrid combinatorial selection approach to generate aptamers probes that have an affinity for EBOV GP. The hybrid SELEX combines two selection approaches to evolve an ssDNA library to bind to the native form of EBOV GP. Initial rounds of the selections are performed on cells with HeLa cells that were transfected with EBOV GP as a selection target. Later rounds of selection switched from cells to beadsimmobilized virus particles that display EBOV GP. I outline my experimental contributions to this ongoing work. The work was done with the help of Dr. David Porciani under the supervision of Dr. Donald Burke.

Chapters 6 is a perspective on utilizing aptamers that bind to virus-cell surfaces as affinity probes for developing low-cost virus biosensors. This perspective was put together with the help of Dr. David Porciani under the supervision of Dr. Burke. This work will be adapted for publication as a review manuscript after further developments. 


\section{Reference:}

(1) Feldmann, H.; Geisbert, T. W. Ebola Haemorrhagic Fever. The Lancet. Lancet Publishing Group 2011, pp 849-862. https://doi.org/10.1016/S01406736(10)60667-8.

(2) Maganga, G. D.; Kapetshi, J.; Berthet, N.; Ilunga, B. K.; Kabange, F.; Kingebeni, P. M.; Mondonge, V.; Muyembe, J. J. T.; Bertherat, E.; Briand, S.; Cabore, J.; Epelboin, A.; Formenty, P.; Kobinger, G.; González-Angulo, L.; Labouba, I.; Manuguerra, J. C.; Okwo-Bele, J. M.; Dye, C.; Leroy, E. M. Ebola Virus Disease in the Democratic Republic of Congo. N. Engl. J. Med. 2014, 371 (22), 20832091. https://doi.org/10.1056/NEJMoa1411099.

(3) Okware, S. I.; Omaswa, F. G.; Zaramba, S.; Opio, A.; Lutwama, J. J.; Kamugisha, J.; Rwaguma, E. B.; Kagwa, P.; Lamunu, M. An Outbreak of Ebola in Uganda. Trop. Med. Int. Heal. 2002, 7 (12), 1068-1075. https://doi.org/10.1046/j.13653156.2002.00944.x.

(4) Ebolavirus and Marburgvirus Infections | Harrison's Principles of Internal Medicine, 19e | AccessMedicine | McGraw-Hill Medical https://accessmedicine.mhmedical.com/content.aspx?bookid=1130\&sectionid=797 39742 (accessed Apr 6, 2020).

(5) Negredo, A.; Palacios, G.; Vázquez-Morón, S.; González, F.; Dopazo, H.; Molero, F.; Juste, J.; Quetglas, J.; Savji, N.; de la Cruz Martínez, M.; Herrera, J. E.; Pizarro, M.; Hutchison, S. K.; Echevarría, J. E.; Lipkin, W. I.; Tenorio, A. Discovery of an Ebolavirus-like Filovirus in Europe. PLoS Pathog. 2011, 7 (10), e1002304. https://doi.org/10.1371/journal.ppat.1002304. 
(6) Beer, B.; Kurth, R.; Bukreyev, A. Characteristics of Filoviridae: Marburg and Ebola Viruses. Naturwissenschaften. January 15, 1999, pp 8-17. https://doi.org/10.1007/s001140050562.

(7) Geisbert, T. W.; Jahrling, P. B. Differentiation of Filoviruses by Electron Microscopy. Virus Res. 1995, 39 (2-3), 129-150. https://doi.org/10.1016/01681702(95)00080-1.

(8) Mühlberger, E. Filovirus Replication and Transcription. Future Virology. NIH Public Access March 2007, pp 205-215. https://doi.org/10.2217/17460794.2.2.205.

(9) Lee, J. E.; Saphire, E. O. Ebolavirus Glycoprotein Structure and Mechanism of Entry. Future Virology. NIH Public Access 2009, pp 621-635. https://doi.org/10.2217/fvl.09.56.

(10) Jasenosky, L. D.; Neumann, G.; Lukashevich, I.; Kawaoka, Y. Ebola Virus VP40Induced Particle Formation and Association with the Lipid Bilayer. J. Virol. 2001, 75 (11), 5205-5214. https://doi.org/10.1128/jvi.75.11.5205-5214.2001.

(11) Adu-Gyamfi, E.; Digman, M. A.; Gratton, E.; Stahelin, R. V. Investigation of Ebola VP40 Assembly and Oligomerization in Live Cells Using Number and Brightness Analysis. Biophys. J. 2012, 102 (11), 2517-2525. https://doi.org/10.1016/j.bpj.2012.04.022.

(12) Soni, S. P.; Adu-Gyamfi, E.; Yong, S. S.; Jee, C. S.; Stahelin, R. V. The Ebola Virus Matrix Protein Deeply Penetrates the Plasma Membrane: An Important Step in Viral Egress. Biophys. J. 2013, 104 (9), 1940-1949.

https://doi.org/10.1016/j.bpj.2013.03.021. 
(13) Basler, C. F.; Mikulasova, A.; Martinez-Sobrido, L.; Paragas, J.; Mühlberger, E.; Bray, M.; Klenk, H.-D.; Palese, P.; García-Sastre, A. The Ebola Virus VP35 Protein Inhibits Activation of Interferon Regulatory Factor 3. J. Virol. 2003, 77 (14), 7945-7956. https://doi.org/10.1128/jvi.77.14.7945-7956.2003.

(14) Bale, S.; Julien, J. P.; Bornholdt, Z. A.; Kimberlin, C. R.; Halfmann, P.; Zandonatti, M. A.; Kunert, J.; Kroon, G. J. A.; Kawaoka, Y.; MacRae, I. J.; Wilson, I. A.; Saphire, E. O. Marburg Virus VP35 Can Both Fully Coat the Backbone and Cap the Ends of DsRNA for Interferon Antagonism. PLoS Pathog. 2012, 8 (9). https://doi.org/10.1371/journal.ppat.1002916.

(15) Watanabe, S.; Noda, T.; Kawaoka, Y. Functional Mapping of the Nucleoprotein of Ebola Virus. J. Virol. 2006, 80 (8), 3743-3751. https://doi.org/10.1128/jvi.80.8.3743-3751.2006.

(16) Kolesnikova, L.; Muhlberger, E.; Ryabchikova, E.; Becker, S. Ultrastructural Organization of Recombinant Marburg Virus Nucleoprotein: Comparison with Marburg Virus Inclusions. J. Virol. 2000, 74 (8), 3899-3904. https://doi.org/10.1128/jvi.74.8.3899-3904.2000.

(17) Pappalardo, M.; Juliá, M.; Howard, M. J.; Rossman, J. S.; Michaelis, M.; Wass, M. N. Conserved Differences in Protein Sequence Determine the Human Pathogenicity of Ebolaviruses. Nat. Publ. Gr. 2016. https://doi.org/10.1038/srep23743.

(18) Sanchez, A.; Trappier, S. G.; Mahy, B. W. J.; Peters, C. J.; Nichol, S. T. The Virion Glycoproteins of Ebola Viruses Are Encoded in Two Reading Frames and Are Expressed through Transcriptional Editing. Proc. Natl. Acad. Sci. U. S. A. 
1996, 93 (8), 3602-3607. https://doi.org/10.1073/pnas.93.8.3602.

(19) Volchkov, V. E.; Feldmann, H.; Volchkova, V. A.; Klenk, H. D. Processing of the Ebola Virus Glycoprotein by the Proprotein Convertase Furin. Proc. Natl. Acad. Sci. U. S. A. 1998, 95 (10), 5762-5767. https://doi.org/10.1073/pnas.95.10.5762.

(20) Shimojima, M.; Takada, A.; Ebihara, H.; Neumann, G.; Fujioka, K.; Irimura, T.; Jones, S.; Feldmann, H.; Kawaoka, Y. Tyro3 Family-Mediated Cell Entry of Ebola and Marburg Viruses. J. Virol. 2006, 80 (20), 10109-10116. https://doi.org/10.1128/JVI.01157-06.

(21) Gramberg, T.; Soilleux, E.; Fisch, T.; Lalor, P. F.; Hofmann, H.; Wheeldon, S.; Cotterill, A.; Wegele, A.; Winkler, T.; Adams, D. H.; Pöhlmann, S. Interactions of LSECtin and DC-SIGN/DC-SIGNR with Viral Ligands: Differential PH Dependence, Internalization and Virion Binding. Virology 2008, 373 (1), 189-201. https://doi.org/10.1016/j.virol.2007.11.001.

(22) Simmons, G.; Reeves, J. D.; Grogan, C. C.; Vandenberghe, L. H.; Baribaud, F.; Whitbeck, J. C.; Burke, E.; Buchmeier, M. J.; Soilleux, E. J.; Riley, J. L.; Doms, R. W.; Bates, P.; Pöhlmann, S. DC-SIGN and DC-SIGNR Bind Ebola Glycoproteins and Enhance Infection of Macrophages and Endothelial Cells. Virology 2003, 305 (1), 115-123. https://doi.org/10.1006/viro.2002.1730.

(23) Alvarez, C. P.; Lasala, F.; Carrillo, J.; Muñiz, O.; Corbí, A. L.; Delgado, R. CType Lectins DC-SIGN and L-SIGN Mediate Cellular Entry by Ebola Virus in Cis and in Trans . J. Virol. 2002, 76 (13), 6841-6844. https://doi.org/10.1128/jvi.76.13.6841-6844.2002.

(24) Kuhn, J. H.; Radoshitzky, S. R.; Guth, A. C.; Warfield, K. L.; Li, W.; Vincent, M. 
J.; Towner, J. S.; Nichol, S. T.; Bavari, S.; Choe, H.; Aman, M. J.; Farzan, M. Conserved Receptor-Binding Domains of Lake Victoria Marburgvirus and Zaire Ebolavirus Bind a Common Receptor. J. Biol. Chem. 2006, 281 (23), 1595115958. https://doi.org/10.1074/jbc.M601796200.

(25) Lee, J. E.; Fusco, M. L.; Hessell, A. J.; Oswald, W. B.; Burton, D. R.; Saphire, E. O. Structure of the Ebola Virus Glycoprotein Bound to an Antibody from a Human Survivor. Nature 2008, 454 (7201), 177-182. https://doi.org/10.1038/nature07082.

(26) Geisbert, T. W.; Hensley, L. E.; Larsen, T.; Young, H. A.; Reed, D. S.; Geisbert, J. B.; Scott, D. P.; Kagan, E.; Jahrling, P. B.; Davis, K. J. Pathogenesis of Ebola Hemorrhagic Fever in Cynomolgus Macaques: Evidence That Dendritic Cells Are Early and Sustained Targets of Infection. Am. J. Pathol. 2003, 163 (6), 2347-2370. https://doi.org/10.1016/S0002-9440(10)63591-2.

(27) Geisbert, T. W.; Young, H. A.; Jahrling, P. B.; Davis, K. J.; Larsen, T.; Kagan, E.; Hensley, L. E. Pathogenesis of Ebola Hemorrhagic Fever in Primate Models: Evidence That Hemorrhage Is Not a Direct Effect of Virus-Induced Cytolysis of Endothelial Cells. Am. J. Pathol. 2003, 163 (6), 2371-2382. https://doi.org/10.1016/S0002-9440(10)63592-4.

(28) Surani, A.; Marfatia, Y.; Pal, A.; Shah, R. Ebola Virus: An Emerging Sexually Transmissible Infection Pathogen. Indian J. Sex. Transm. Dis. AIDS 2018, 39 (1), 65. https://doi.org/10.4103/ijstd.ijstd_21_18.

(29) $\mathrm{WHO} \mid$ Interim Advice on the Sexual Transmission of the Ebola Virus Disease. WHO 2016.

(30) Guidance on Personal Protective Equipment (PPE) | Personal Protective 
Equipment (PPE) | Public Health Planners | Ebola (Ebola Virus Disease) | CDC https://www.cdc.gov/vhf/ebola/healthcare-us/ppe/guidance.html (accessed Apr 7, 2020).

(31) Baize, S.; Leroy, E. M.; Georges, A. J.; Georges-Courbot, M. C.; Capron, M.; Bedjabaga, I.; Lansoud-Soukate, J.; Mavoungou, E. Inflammatory Responses in Ebola Virus-Infected Patients. Clin. Exp. Immunol. 2002, 128 (1), 163-168. https://doi.org/10.1046/j.1365-2249.2002.01800.x.

(32) Wauquier, N.; Becquart, P.; Padilla, C.; Baize, S.; Leroy, E. M. Human Fatal Zaire Ebola Virus Infection Is Associated with an Aberrant Innate Immunity and with Massive Lymphocyte Apoptosis. PLoS Negl. Trop. Dis. 2010, 4 (10). https://doi.org/10.1371/journal.pntd.0000837.

(33) Ebihara, H.; Rockx, B.; Marzi, A.; Feldmann, F.; Haddock, E.; Brining, D.; LaCasse, R. A.; Gardner, D.; Feldmann, H. Host Response Dynamics Following Lethal Infection of Rhesus Macaques with Zaire Ebolavirus. J. Infect. Dis. 2011, 204 Suppl 3, S991-9. https://doi.org/10.1093/infdis/jir336.

(34) Hensley, L. E.; Young, H. A.; Jahrling, P. B.; Geisbert, T. W. Proinflammatory Response during Ebola Virus Infection of Primate Models: Possible Involvement of the Tumor Necrosis Factor Receptor Superfamily. Immunol. Lett. 2002, 80 (3), 169-179. https://doi.org/10.1016/S0165-2478(01)00327-3.

(35) Sanchez, A.; Lukwiya, M.; Bausch, D.; Mahanty, S.; Sanchez, A. J.; Wagoner, K. D.; Rollin, P. E. Analysis of Human Peripheral Blood Samples from Fatal and Nonfatal Cases of Ebola (Sudan) Hemorrhagic Fever: Cellular Responses, Virus Load, and Nitric Oxide Levels. J. Virol. 2004, 78 (19), 10370-10377. 
https://doi.org/10.1128/JVI.78.19.10370-10377.2004.

(36) Gear, J. H. Clinical Aspects of African Viral Hemorrhagic Fevers. Rev. Infect. Dis. 11 Suppl 4, S777-82. https://doi.org/10.1093/clinids/11.supplement_4.s777.

(37) Martini, G. A.; Knauff, H. G.; Schmidt, H. A.; Mayer, G.; Baltzer, G. A Hitherto Unknown Infectious Disease Contracted from Monkeys. "Marburg-Virus" Disease. Ger. Med. Mon. 1968, 13 (10), 457-470.

(38) Languon, S.; Quaye, O. Filovirus Disease Outbreaks: A Chronological Overview. Virology: Research and Treatment. SAGE Publications Ltd June 1, 2019. https://doi.org/10.1177/1178122X19849927.

(39) $\mathrm{WHO} \mid$ Case of Marburg Haemorrhagic Fever Imported into the Netherlands from Uganda. WHO 2015.

(40) Regules, J. A.; Beigel, J. H.; Paolino, K. M.; Voell, J.; Castellano, A. R.; Hu, Z.; Muñoz, P.; Moon, J. E.; Ruck, R. C.; Bennett, J. W.; Twomey, P. S.; Gutiérrez, R. L.; Remich, S. A.; Hack, H. R.; Wisniewski, M. L.; Josleyn, M. D.; Kwilas, S. A.; Van Deusen, N.; Mbaya, O. T.; Zhou, Y.; Stanley, D. A.; Jing, W.; Smith, K. S.; Shi, M.; Ledgerwood, J. E.; Graham, B. S.; Sullivan, N. J.; Jagodzinski, L. L.; Peel, S. A.; Alimonti, J. B.; Hooper, J. W.; Silvera, P. M.; Martin, B. K.; Monath, T. P.; Ramsey, W. J.; Link, C. J.; Lane, H. C.; Michael, N. L.; Davey, R. T.; Thomas, S. J. A Recombinant Vesicular Stomatitis Virus Ebola Vaccine. N. Engl. J. Med. 2017, 376 (4), 330-341. https://doi.org/10.1056/NEJMoa1414216.

(41) Geisbert, T. W. First Ebola Virus Vaccine to Protect Human Beings? The Lancet. Lancet Publishing Group February 4, 2017, pp 479-480. https://doi.org/10.1016/S0140-6736(16)32618-6. 
(42) Geisbert, T.; Daddario-Dicaprio, K.; Lewis, M.; al., et. Vesicular Stomatitis VirusBased Ebola Vaccine Is Well-Tolerated and Protects Immunocompromised NonHuman Primates. PLoS Pathog 2008, 4, e1000225.

(43) First FDA-approved vaccine for the prevention of Ebola virus disease, marking a critical milestone in public health preparedness and response | FDA https://www.fda.gov/news-events/press-announcements/first-fda-approvedvaccine-prevention-ebola-virus-disease-marking-critical-milestone-public-health (accessed Apr 7, 2020).

(44) Sheridan, C. Merck Vaccine Heads Ebola Countermeasures. Nat. Biotechnol. 2018, 36 (7), 563-565. https://doi.org/10.1038/nbt0718-563a.

(45) First Ebola Vaccine Approved. Nature biotechnology. NLM (Medline) January 1, 2020, p 6. https://doi.org/10.1038/s41587-019-0385-7.

(46) Pascal, K. E.; Dudgeon, D.; Trefry, J. C.; Anantpadma, M.; Sakurai, Y.; Murin, C. D.; Turner, H. L.; Fairhurst, J.; Torres, M.; Rafique, A.; Yan, Y.; Badithe, A.; Yu, K.; Potocky, T.; Bixler, S. L.; Chance, T. B.; Pratt, W. D.; Rossi, F. D.; Shamblin, J. D.; Wollen, S. E.; Zelko, J. M.; Carrion, R.; Worwa, G.; Staples, H. M.; Burakov, D.; Babb, R.; Chen, G.; Martin, J.; Huang, T. T.; Erlandson, K.; Willis, M. S.; Armstrong, K.; Dreier, T. M.; Ward, A. B.; Davey, R. A.; Pitt, M. L. M.; Lipsich, L.; Mason, P.; Olson, W.; Stahl, N.; Kyratsous, C. A. Development of Clinical-Stage Human Monoclonal Antibodies That Treat Advanced Ebola Virus Disease in Nonhuman Primates. J. Infect. Dis. 612 (5), 218. https://doi.org/10.1093/infdis/jiy285.

(47) Independent Monitoring Board Recommends Early Termination of Ebola 
Therapeutics Trial in DRC Because of Favorable Results with Two of Four Candidates | NIH: National Institute of Allergy and Infectious Diseases https://www.niaid.nih.gov/news-events/independent-monitoring-boardrecommends-early-termination-ebola-therapeutics-trial-drc (accessed Apr 7, 2020).

(48) Lucey, D. R. New Treatments for Ebola Virus Disease. The BMJ. BMJ Publishing Group September 6, 2019. https://doi.org/10.1136/bmj.15371. Dyer, O. Two Ebola Treatments Halve Deaths in Trial in DRC Outbreak. BMJ 2019, 366, 15140. https://doi.org/10.1136/bmj.15140.

(50) Gilchuk, P.; Murin, C. D.; Milligan, J. C.; Cross, R. W.; Mire, C. E.; Ilinykh, P. A.; Huang, K.; Kuzmina, N.; Altman, P. X.; Hui, S.; Gunn, B. M.; Bryan, A. L.; Davidson, E.; Doranz, B. J.; Turner, H. L.; Alkutkar, T.; Flinko, R.; Orlandi, C.; Carnahan, R.; Nargi, R.; Bombardi, R. G.; Vodzak, M. E.; Li, S.; Okoli, A.; Ibeawuchi, M.; Ohiaeri, B.; Lewis, G. K.; Alter, G.; Bukreyev, A.; Saphire, E. O.; Geisbert, T. W.; Ward, A. B.; Crowe, J. E. Analysis of a Therapeutic Antibody Cocktail Reveals Determinants for Cooperative and Broad Ebolavirus Neutralization. Immunity 2020, 52 (2), 388-403.e12. https://doi.org/10.1016/j.immuni.2020.01.001.

(51) Mulangu, S.; Dodd, L. E.; Davey, R. T.; Tshiani Mbaya, O.; Proschan, M.; Mukadi, D.; Lusakibanza Manzo, M.; Nzolo, D.; Tshomba Oloma, A.; Ibanda, A.; Ali, R.; Coulibaly, S.; Levine, A. C.; Grais, R.; Diaz, J.; Lane, H. C.; MuyembeTamfum, J.-J.; the PALM Writing Group. A Randomized, Controlled Trial of Ebola Virus Disease Therapeutics. N. Engl. J. Med. 2019, 381 (24), 2293-2303. https://doi.org/10.1056/NEJMoa1910993. 
(52) Fischer, W. A.; Vetter, P.; Bausch, D. G.; Burgess, T.; Davey, R. T.; Fowler, R.; Hayden, F. G.; Jahrling, P. B.; Kalil, A. C.; Mayers, D. L.; Mehta, A. K.; Uyeki, T. M.; Jacobs, M. Ebola Virus Disease: An Update on Post-Exposure Prophylaxis. The Lancet Infectious Diseases. Lancet Publishing Group June 1, 2018, pp e183e192. https://doi.org/10.1016/S1473-3099(17)30677-1.

(53) Thomson, E. C.; Davis, C.; Tipton, T.; Sabir, S.; Aitken, C.; Bennett, S.; Becker, S.; Evans, T.; Fehling, S. K.; Gunson, R.; Hall, Y.; Jackson, C.; Johanssen, I.; Kieny, M. P.; Mcmenamin, J.; Spence, E.; Strecker, T.; Sykes, C.; Templeton, K.; Thorburn, F.; Peters, E.; Maria, A.; Restrepo, H.; White, B.; Zambon, M.; Carroll, M. W.; Thomson, E. C. Clinical Infectious Diseases Clinical Infectious Diseases ${ }^{\circledR}$ 2019;XX(XX):1-8 Postexposure Prophylaxis With RVSV-ZEBOV Following Exposure to a Patient With Ebola Virus Disease Relapse in the United Kingdom: An Operational, Safety, and Immunogenicity Report. 2019. https://doi.org/10.1093/cid/ciz1165.

(54) Tembo, J.; Simulundu, E.; Changula, K.; Handley, D.; Gilbert, M.; Chilufya, M.; Asogun, D.; Ansumana, R.; Kapata, N.; Ntoumi, F.; Ippolito, G.; Zumla, A.; Bates, M. Recent Advances in the Development and Evaluation of Molecular Diagnostics for Ebola Virus Disease. Expert Review of Molecular Diagnostics. Taylor and Francis Ltd April 3, 2019, pp 325-340. https://doi.org/10.1080/14737159.2019.1595592.

(55) Wonderly, B.; Jones, S.; Gatton, M. L.; Barber, J.; Killip, M.; Hudson, C.; Carter, L.; Brooks, T.; Simpson, A. J. H.; Semper, A.; Urassa, W.; Chua, A.; Perkins, M.; Boehme, C. Comparative Performance of Four Rapid Ebola Antigen-Detection 
Lateral Flow Immunoassays during the 2014-2016 Ebola Epidemic in West Africa. PLoS One 2019, 14 (3), e0212113. https://doi.org/10.1371/journal.pone.0212113.

(56) Ebola virus disease https://www.who.int/news-room/fact-sheets/detail/ebola-virusdisease (accessed Apr 7, 2020).

(57) Marburg virus disease https://www.who.int/news-room/fact-sheets/detail/marburgvirus-disease (accessed Apr 7, 2020).

(58) Phillips, C. Letter of Authorization for BioFire FilmArray Biothreat-E Test; 2014.

(59) Shurtleff, A. C.; Whitehouse, C. A.; Ward, M. D.; Cazares, L. H.; Bavari, S. PreSymptomatic Diagnosis and Treatment of Filovirus Diseases. Frontiers in Microbiology. Frontiers Media S.A. 2015. https://doi.org/10.3389/fmicb.2015.00108.

(60) Towner, J. S.; Rollin, P. E.; Bausch, D. G.; Sanchez, A.; Crary, S. M.; Vincent, M.; Lee, W. F.; Spiropoulou, C. F.; Ksiazek, T. G.; Lukwiya, M.; Kaducu, F.; Downing, R.; Nichol, S. T. Rapid Diagnosis of Ebola Hemorrhagic Fever by Reverse Transcription-PCR in an Outbreak Setting and Assessment of Patient Viral Load as a Predictor of Outcome. J. Virol. 2004, 78 (8), 4330-4341. https://doi.org/10.1128/jvi.78.8.4330-4341.2004.

(61) Zhou, J.; Rossi, J. Aptamers as Targeted Therapeutics: Current Potential and Challenges. Nat Rev Drug Discov 2016, advance on . https://doi.org/10.1038/nrd.2016.199.

(62) Tawiah, K. D.; Porciani, D.; Burke, D. H. Toward the Selection of Cell Targeting Aptamers with Extended Biological Functionalities to Facilitate Endosomal Escape of Cargoes. Biomedicines. MDPI AG September 1, 2017, p 51. 
https://doi.org/10.3390/biomedicines5030051. 


\title{
Chapter 2: Toward the Selection of Cell Targeting Aptamers with Extended Biological Functionalities to Facilitate Endosomal Escape of Cargoes
}

As found in: Tawiah, K. D.; Porciani, D.; Burke, D. H. Towards the Selection of Cell Targeting Aptamers with Extended Biological Functionalities to Facilitate Endosomal Escape of Cargoes. Biomedicines 2017, 5 (3). doi.org/10.3390/biomedicines5030051

\begin{abstract}
Over the past decades there have been exciting and rapid developments of highly specific molecules to bind cancer antigens that are overexpressed on the surfaces of malignant cells. Nanomedicine aims to exploit these ligands to generate nanoscale platforms for targeted cancer therapy, and to do so with negligible off-target effects. Aptamers are structured nucleic acids that bind to defined molecular targets ranging from small molecules and proteins to whole cells or viruses. They are selected through an iterative process of amplification and enrichment called SELEX, (systematic evolution of ligands by exponential enrichment) in which a combinatorial oligonucleotide library is exposed to the target of interest for several repetitive rounds. Nucleic acid ligands able to bind and internalize into malignant cells have been extensively used as tools for targeted delivery of therapeutic payloads both in vitro and in vivo. However, current cell targeting aptamer platforms suffer from limitations that have slowed their translation to the clinic. This is especially true for applications in which the cargo must reach the cytosol to exert its biological activity, as only a small percentage of the endocytosed cargo is typically able
\end{abstract}


to translocate into the cytosol. Innovative technologies and selection strategies are required to enhance cytoplasmic delivery. In this review, we describe current selection methods used to generate aptamers that target cancer cells, and we highlight some of the factors that affect productive endosomal escape of cargoes. We also give an overview of the most promising strategies utilized to improve and monitor endosomal escape of therapeutic cargoes. The methods we highlight exploit tools and technologies that can potentially be incorporated in the SELEX process. Innovative selection protocols may identify aptamers with extended biological functionalities that allow effective cytosolic translocation of therapeutics. This in turn may facilitate successful translation of these platforms into clinical applications.

\section{Introduction}

Cancer remains one of the leading causes of death worldwide, with high morbidity and mortality. Though significant advances have been made in the fundamental understanding of cancer biology, the treatment of some cancer forms remains elusive, and many cancer treatments suffer from severe side effects ${ }^{1,2}$. Over the past decades, novel active biomolecules such as regulatory peptides or short interfering RNAs are emerging as promising agents for cancer therapy and for studying tissue-specific gene function ${ }^{3-5}$. However, they possess some limitations. In particular, siRNAs and most regulatory peptides are relatively large, highly polar and charged molecules that require delivery vehicles to cross the plasma membrane. Promising delivery strategies have been developed in the form of lipid and polymeric nanoparticles ${ }^{6,7}$. However, these strategies usually lack selectivity and are unable to discriminate between tumor and healthy cells, 
causing off-target effects ${ }^{8}$. In light of these considerations, significant efforts have been devoted to developing effective targeted delivery nanostructures able to direct these therapeutics specifically toward cancer cells ${ }^{8,9}$. A common strategy to enhance selectivity relies on developing ligands that bind biomolecules that are overexpressed on the surfaces of malignant cells. To date, the most used recognition tools are proteins (antibodies and their fragments) ${ }^{10,11}$, peptides ${ }^{11}$, oligonucleotides (nucleic acid aptamers) ${ }^{12}$, and small molecules (vitamins, carbohydrates) ${ }^{13}$. Several prior reviews have highlighted the structural requirements (size and charge) and pharmacokinetic features that a ligand-drug platform should possess to minimize side effects and increase its therapeutic window ${ }^{2,8}$. However, only a few studies have performed a side-by-side analysis in vitro and in vivo to compare selectivity of these classes of ligands ${ }^{14}$. Aptamers are short, single-chained DNA or RNA molecules that recognize molecular targets with high affinity (in $\mathrm{nM}$ to $\mathrm{pM}$ range) and specificity owing to their 3D conformation. Aptamers are emerging as a class of biocompatible ligands with huge potential in diagnostics and therapeutics ${ }^{15-17}$. Compared with antibodies, aptamer production exploits a chemical technology with high batch fidelity that is not intrinsically prone to viral or bacterial contamination ${ }^{15}$. In addition, the process of chemical synthesis allows the site-specific introduction of non-nucleotide linkers (e.g., hexaethylene glycol), or chemical functionalities useful for conjugation (such as primary amines, thiols, and terminal alkynes), and the addition of fluorescent or other reporter moieties. Moreover, due to their small sizes (aptamers: $25-70 \mathrm{nt}$ equals 8-20 kDa; common antibodies $\sim 150$ $160 \mathrm{kDa}$; Fab fragments $\sim 50 \mathrm{kDa}$; and single chain variable fragments $\sim 25 \mathrm{kDa})^{1}$, aptamers can penetrate into biological compartments more efficiently than common 
antibodies and most of the engineered antibody fragments, and they can potentially access protein structural domains that might be sterically inaccessible to bulkier ligands 15 .

Despite the rapid development of diverse platforms that exploit aptamers to cell-surface receptors as vehicles to deliver peptides and siRNAs, none has made it into clinical trials yet ${ }^{15}$. Some of the translational bottlenecks are related to current technological limitations. For instance, even when individual aptamers can be readily synthesized, elaborating them into functionalized nanoparticles (such as polymeric nanoparticles, micelles, and liposomes) tends to be tedious and expensive with low yields and difficult scale-up ${ }^{18}$. Recently, Urmann et al. highlighted some of these limitations and offered an insight on potential strategies to tackle these barriers ${ }^{19}$. Although advances in nanotechnology might overcome these constraints, additional limitations need to be addressed to allow for an efficient transition to clinical application. Key among these is the productive engagement of biological mechanisms that govern the processing of these aptamer-based delivery platforms upon internalization into target cells. These nanostructures internalize through an endocytosis mechanism (generally clathrinmediated) and subsequently are concentrated in endocytic vesicles such as endosomes. At this stage, without any aid to escape from the endosomes, siRNA and peptide cargoes with polar and charged character and large size cannot translocate efficiently into the cytosol to exhibit their biological activity, and they instead remain entrapped in this compartment ${ }^{6,20}$. The amount of therapeutic cargo that can successfully access the cytosol appears to depend mainly on three factors: the number of targeted receptor molecules on the cell surface, the rates of their endocytic uptake, and the endosomal 
escape efficiency over the course of a cycle of internalization. Notably, some receptors should also possess a fast recycling to restore their high levels on the cell-surface and ensure continuous uptake of cargoes. As shown in Fig 2.1, improving any one of these levels can lead to improved net endosomal escape, greater cytosolic accessibility, and consequently to more effective biological activity. 
a

Low receptor expression $\left(<10^{4}\right)$

Cinum

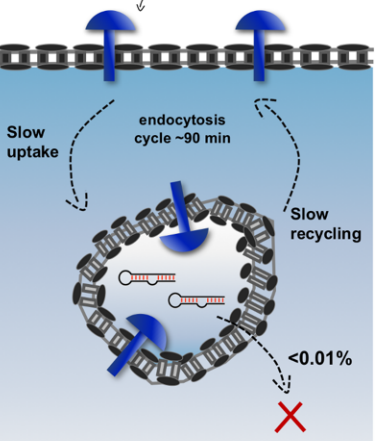

Low efficiency of escape b

High receptor expression $\left(>10^{5}\right)$

Cincm

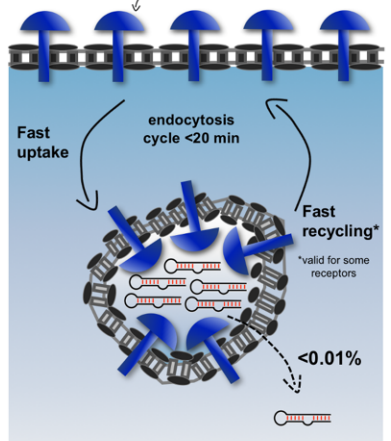

Low efficiency of escape
C High receptor expression $\left(>10^{5}\right)$

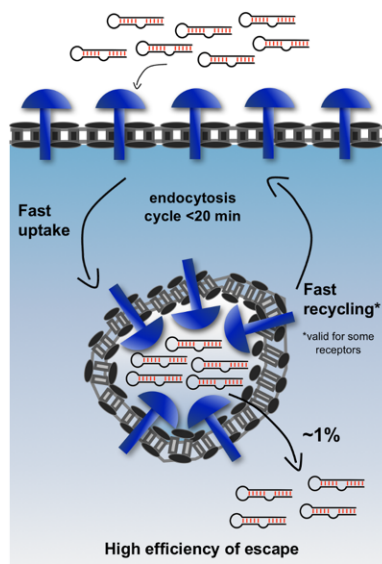

Figure 2.1. Relationships among three key factors that govern cytosolic delivery: receptor expression on the cell surface, rate of endocytosis and endosomal escape efficiency of cargoes. In (A, B) the estimated efficiency of escape is $<0.01 \%$ per cycle of endocytosis; A. Upon binding to low-expressed receptors $\left(<10^{4}\right.$ copies/cell) that internalize with slow rate of uptake ( $99 \mathrm{~min})$, a negligible cytoplasmic translocation of internalized aptamers occurs; B. Increased translocation can be observed when cell targeting aptamers bind highly expressed receptors $\left(>10^{5}\right.$ copies/cell $)$ that show faster internalization $(\sim 20 \mathrm{~min})$. This situation can, in some extent, resemble some successful cancer therapy strategies aim to target asialoglycoprotein receptor (ASGPR), which is expressed at high levels on the surface of liver cells C. An ideal delivery system should bind highly expressed receptors $\left(>10^{5}\right)$ with high rate of uptake ( 20 $\mathrm{min})$ and should possess an enhanced endosomal escape efficiency (i.e., high efficiency of cargoes $\sim 1 \%$ /cycle).

2. Figure 2.2 
The quantitative impacts of these factors can be illustrated by doing an admittedly oversimplified calculation. Recent studies suggest that significant siRNA-mediated gene knock-down requires approximately $\sim 2000-5000$ siRNA molecules in the cytosol ${ }^{20,21}$. Current estimates are that $<0.01 \%$ of the internalized molecules successfully translocate into the cytosol during each cycle of internalization ${ }^{20}$. The majority of targetable cellsurface markers are expressed at roughly $10^{4}-10^{5}$ copies/cell ${ }^{6}$. If targeting ligands carrying siRNA cargo were able to bind all of those receptors and were then all internalized, $10^{4}-10^{5}$ of siRNAs would be delivered into endosomes during the course of one cycle of endocytosis, but only one to ten of those molecules $(0.01 \%)$ would escape into the cytosol in that cycle. Given that plasma receptors typically recycle every 90 minutes for the major routes of internalization (caveolin and clathrin-mediated endocytosis), 500-5000 cycles of endocytosis (750-7500 h) would be required to deliver $\sim 5000$ siRNAs into the cytosol! In principle, the required time scale could be dramatically shortened by targeting receptors that have an expression $>10^{5}$ (but they are very rare) with fast uptake ( $<20 \mathrm{~min})$, increasing the escape efficiency and delivering multiple cargo molecules for each receptor binding event (e.g., micelles or lipid nanoparticles). Using this simplified framework, an improved platform carrying 10 cargo molecules that binds $10^{5}$ cell-surface receptors, internalizes rapidly and escapes from endosomes with an efficiency of $1 \%$ per cycle could quickly deliver 5000 siRNAs to the cytosol. In fact, many of the successful demonstrations of targeted delivery in vivo have exploited one or more of these strategies. For example, the asialoglycoprotein receptor (ASGPR) on liver cells and prostate-specific membrane antigen (PSMA) on prostate cancer cell lines are both expressed at very high levels $\left(\sim 10^{6}\right.$ copies/cell $)$, and internalize 
rapidly (15min) ${ }^{20,22,23}$. However, these examples are, to some extent, the low-hanging fruit of the field. Extending targeted delivery to cells that display surface proteins at more typical levels will require technological improvements. Notably, the majority of targeted receptors are expressed also on the surface of healthy cells, albeit at lower levels $\left(\sim 10^{3}\right.$ copies/cell). Even considering an efficiency of endosomal escape of $1 \%$ per cycle, the translocation of 5000 siRNAs in the cytosol of noncancerous cells would take a considerable amount of time ( $\sim 1$ week), thus reducing the risk of off target effects. Here, in this review, we provide a comprehensive overview of the creative approaches being adopted to generate cell targeting aptamers. We describe recent strategies that lead to the selection of nucleic acid ligands with improved targeting properties and internalization abilities. In addition, we discuss the advantages and limitations of the most promising methods utilized to improve and monitor endosomal escape of therapeutic cargoes. An exciting frontier could be to incorporate these strategies into aptamer selection protocols to identify cell targeting aptamers that ensure improved cytoplasmic accessibility of their cargoes.

\section{In Vitro Selection of Cell Targeting Aptamers}

Aptamers are generated using a selection strategy called SELEX (Systematic Evolution of Ligands by Exponential enrichment), an iterative process of exponential enrichment and amplification of desired sequences from a large combinatorial library $\left(\sim 10^{14}\right.$ species $)$ 24,25 . In each cycle, the evolving library is exposed to the target of interest and subjected to selection pressures that enrich nucleic acid molecules that bind the desired target. Since the invention of SELEX, a plethora of high-affinity RNA and DNA aptamers have 
been selected toward a wide range of different targets ranging from small molecules (i.e. metal ions, organic dyes, amino acids, and peptides $)^{26}$, to proteins ${ }^{27}$, to complex targets (whole cells, viruses or bacteria) ${ }^{15}$. To date, only one aptamer (Pegaptanib, Macugen ${ }^{\circledR}$ ) has been approved by the Food and Drug Administration in 2004 for the treatment of age-related macular degeneration (AMD) ${ }^{28}$. Pegaptanib is an RNA aptamer that specifically recognizes with high affinity (Kd $\sim 50 \mathrm{pM})$ and antagonizes the human vascular endothelial growth factor( $\left.\mathrm{VEGF}_{165}\right)^{29}$. Other therapeutic aptamers are currently being evaluated in various clinical trials, ranging from phase one to three. Recent reviews detail the advances of these aptamer nanomedicines in clinical trials ${ }^{30,31}$. Many different SELEX protocols have been employed to generate aptamers with effective cell-targeting and internalizing properties, most of which reflect two main approaches: traditional protein-based SELEX for binding to purified membrane proteins and live cell-based SELEX ${ }^{32,33}$. Some of the most used aptamers that target cancer antigens are listed in Table 1. 
Table 2.1. Aptamers that bind cell-surface markers selected by protein-SELEX or cell-SELEX

\begin{tabular}{ccccc}
\hline Aptamer Library & Name of the & Biomarker & Type of SELEX & References \\
& Aptamer & & & \\
\hline 2'F-RNA & E07 & EGFR & Protein-SELEX & 34 \\
DNA & Sgc8 & PTK7 & Cell SELEX & 35 \\
Thio-DNA & TA1-TA6 & CD44 & Protein-SELEX & 36,37 \\
2'F-RNA & c2, Waz & CD71 (hTfR) & Hybrid SELEX ${ }^{1}$ & 38,39 \\
2'F-RNA & A9, A10 & PSMA & Protein-SELEX & 40 \\
DNA & AS1411 & Nucleolin & screening of G-rich & \\
& & & oligos in cell lines & \\
DNA & C10, C10.36 & CD19 (+) Burkitt & Cell SELEX & 44,45 \\
2'F-RNA & B1, C1, E1 & lymphoma & & 46 \\
RNA, DNA & FB4, GS24 (DW4) & HER2 & Cell SELEX & 46 \\
2'F-RNA & GL56 & Insulin receptor & Cell SELEX & 49 \\
\hline
\end{tabular}


As shown in Fig 2.2, in a typical protein-based SELEX, the nucleic acid library is mixed with recombinant purified target protein, such as the soluble domain of a cell surface antigen (depicted in blue) that is overexpressed and/or mutated in diseased cells.

Traditional bead-, resin-, membrane-, or chip-based partition approaches are employed to separate target-bound sequences from unbound species ${ }^{50}$. These strategies allow for low non-specific binding and easy control of the conditions. For example, Lupold et al.

successfully selected two 2'-fluoro-modified RNA aptamers (termed A9 and A10) using a purified fusion protein containing the extracellular portion of the PSMA immobilized on magnetic beads ${ }^{40}$. 


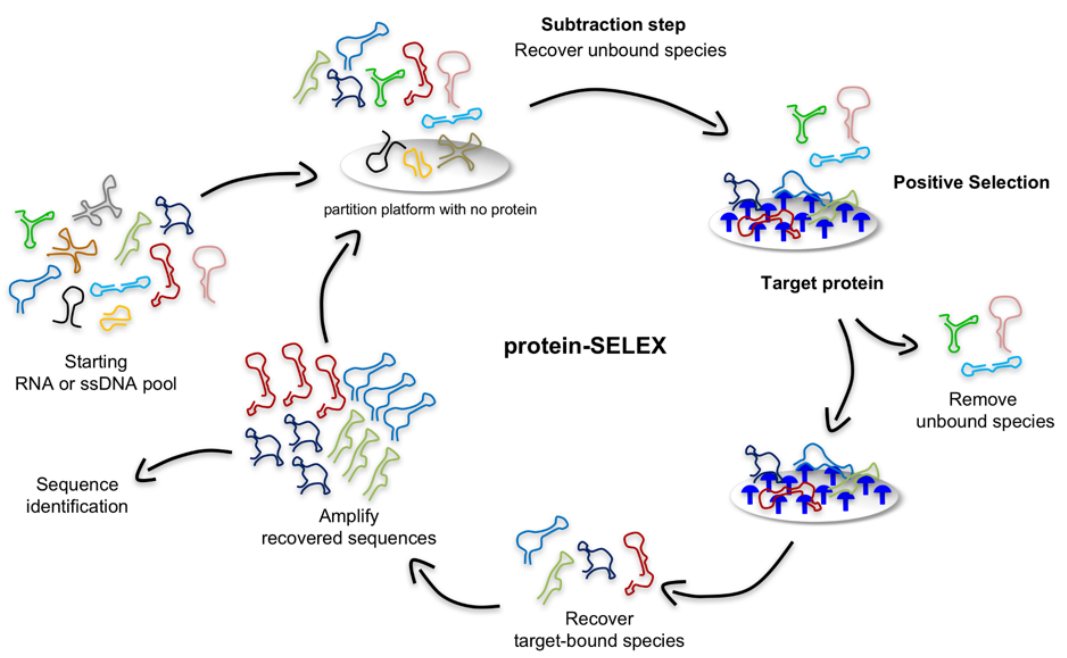

Figure 2.3. Schematic illustration of the protein-based SELEX. In a typical protocol, a subtractive step is first performed to remove nucleic acid sequences with significant affinity toward the partition platform used during the SELEX (e.g., beads, nitrocellulose filter, or chip-based approaches). The recovered unbound sequences are then incubated with the target protein (depicted in blue), and target-bound ligands are separated from the unbound population, recovered and amplified for the next SELEX round. 
Although, most cell targeting aptamers have been generated using protein-based selections, recombinant purified proteins may not fold into the correct $3 \mathrm{D}$ structure that is formed under physiologic conditions ${ }^{51}$. In most cases, only the extracellular domain of a cell-surface receptor is used as target during the selection and the absence of a transmembrane domain may alter the overall 3D conformation. Moreover, recombinant proteins are generated through in vitro expression systems, which often do not include the presence of post-translational modifications. Additionally, in some cases cell-surface proteins are insoluble in their recombinant forms, require interactions with other cell components (e.g., G protein-coupled receptor), or form multimeric and/or multivalent structures ${ }^{52}$. Thus, RNA and DNA aptamers selected through a protein-based SELEX approach might not be able to recognize the same target when embedded in a physiological milieu. For instance, the Sullenger group selected RNA aptamers against the histidine-tagged epidermal growth factor receptor (EGFR)vIII ectodomain ${ }^{53}$. This recombinant protein was expressed and purified using an Escherichia coli system, which lacks post-translational modifications. Selected aptamers were not able to bind the same protein when it was expressed in eukaryotic cells, which the authors concluded is due to the lack of one specific post-translation modification (i.e., glycosylation), significantly altering the structure of the target protein ${ }^{53}$. Live cell-based SELEX (or cell SELEX) overcomes these limitations by using whole living cells as the selection target so that surface antigens are displayed in a more native environment (Fig 2.3). In contrast to the protein-based SELEX, cell SELEX does not need information regarding native conformation or biological function of target proteins, and aptamers can even be generated against unknown cell-surface antigens. This method relies on the difference 
between the expression pattern of cell-surface receptors in target cell population (e.g., cancer cells) and the receptor pattern in control cell line (e.g., healthy cells) ${ }^{35}$. Thereby, a counter selection (or subtractive selection) is implemented in early rounds of the cell SELEX to exclude sequences that have an affinity for components present on the surface of both target and non-target cells. As shown in Fig 2.3, during this subtractive step, the library is incubated with a control cell line and the unbound sequences are recovered and subsequently incubated with target cells. 


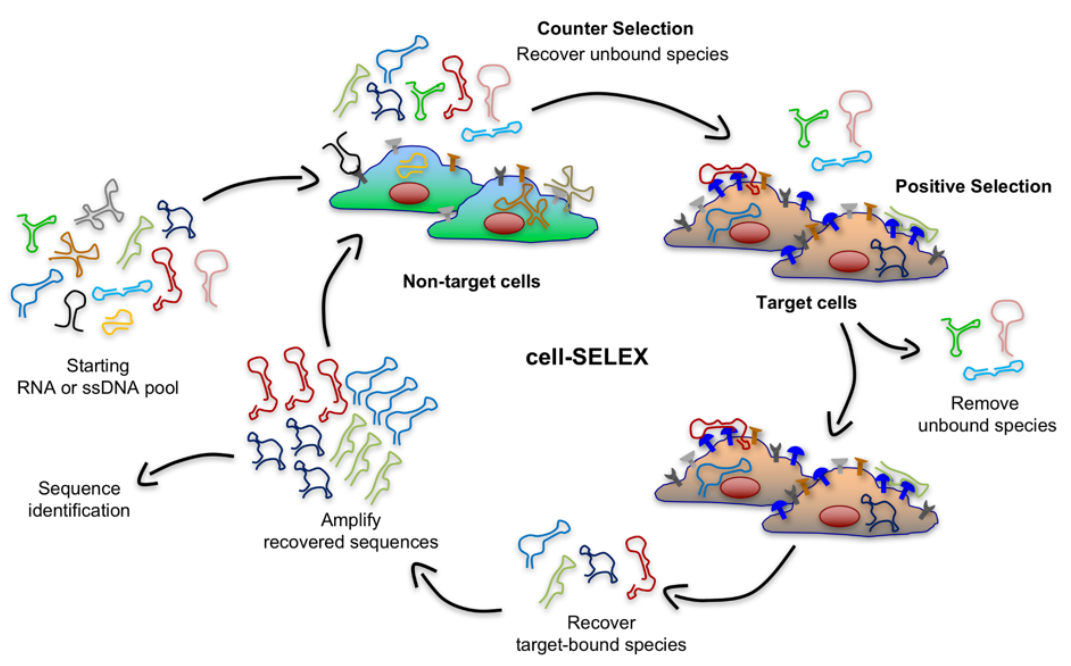

Figure 2.4. Schematic illustration of the cell-SELEX. This procedure consists of four main steps for each round of selection: (i) counter-selection by incubating the nucleic acid library with negative cells (green cells) that do not express target antigens, (ii) a positive selection by incubating recovered unbound sequences with positive cells (tan cells) expressing cell-surface antigens (depicted in blue), (iii) recovery of targetbound sequences, and finally (iv) re-amplification of recovered species. 
Aptamers from cell SELEX can be used to identify novel tumor-associated biomarkers.

For example, aptamers that recognize specific tumor cells can be used as affinity capture reagents to isolate their biomolecular targets ${ }^{33,54}$, followed by liquid chromatographymass spectroscopy (LC-MS) for target identification ${ }^{33}$. Confirmation of the target identity can be further assessed by testing the cell binding properties of aptamers toward a cell line in which the expression of the target gene is silenced either via an RNAi or CRISPR-Cas9 system ${ }^{55}$. Cell SELEX therefore offers tremendous advantages to generate aptamers potentially usable in clinical applications. However, it is also relatively complex and often requires more rounds of positive selection compared to protein-SELEX. In fact, the lack of knowledge about identity and expression level of the biomarker of interest might result in the enrichment of many unrelated/unwanted aptamers binding off-target surface molecules co-expressed on target cells. Therefore, more rounds of counter selection are required to improve the selectivity of aptamers.

\section{Recent Advances in Cell-internalization SELEX}

An ideal platform for targeted delivery should possess a high rate of endocytosis upon the binding of a highly expressed receptor. Recent years have seen the emergence of improved and creative SELEX approaches to generate targeting aptamers that rapidly internalize into target cells. These strategies go beyond simply finding aptamers that bind a given cell by depleting sequences that lack internalization ability or that are endocytosed slowly while enriching sequences that rapidly internalize upon binding with a biomarker on the surface of target cells. To accomplish this, the Giangrande group pioneered a modified cell SELEX methodology that they termed cell-internalization SELEX, in which 
they introduced a stringent, high-salt wash of target cells after incubation with the RNA library ${ }^{46}$. As shown in Fig 2.4, this approach enables the recovery of nucleic acid sequences that rapidly internalize while discarding all non-internalizing cell surface binders and those that internalize with a slower rate because of the properties of the cellsurface receptor they recognize or the mode of interaction. Notably, Thiel et al. generated aptamers that were effectively endocytosed into target cells upon binding with human epidermal growth factor receptor 2 (EGFR2 or HER2), a transmembrane protein overexpressed in breast cancer cells. The fast uptake and targeting properties of these nucleic acid ligands were then exploited to deliver siRNAs targeting Bcl2 gene in breast cancer cells ${ }^{46}$. 


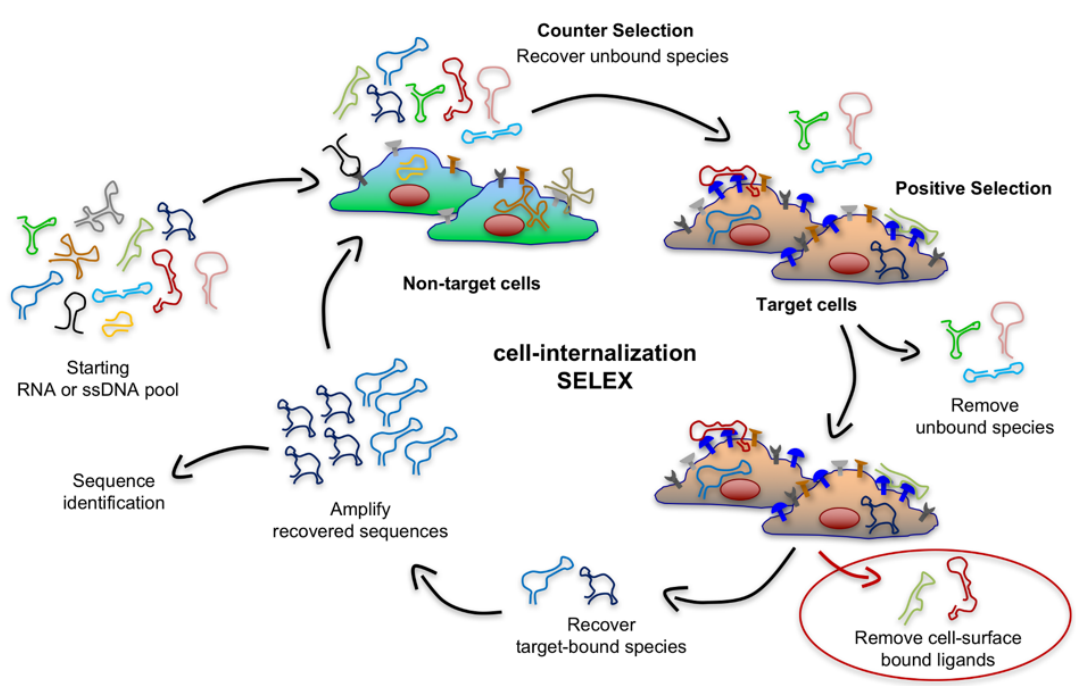

Figure 2.5. Schematic illustration of the cell-internalization SELEX.This modified selection protocol aims at selecting nucleic acid ligands that are able to both bind cell-surface antigens (depicted in blue) and their ability to rapidly internalize into the cell under physiological conditions, while effectively eliminating aptamers that lack internalizing ability and aptamers that only slowly internalize into the cells because of the target they bind. 
Later reports highlighted that the stringent, high-salt wash alone was not sufficient to remove the totality of non-internalizing sequences bound to the cell surface. A second strategy, introduced by Levy group, exploits a cocktail of RNases to digest cell surface bound sequences. This group used aggressive nuclease treatment in several selections to generate 2'-fluoropyrimidine-containing RNA aptamers that readily internalize into various target cells ${ }^{38,56}$. In particular, the $\mathrm{C} 1$ and Otter aptamers were able to penetrate into a diverse collection of mouse and human cells, including primary cells ${ }^{56}$. These aptamers are generalists with limited specificity, so they are not ideal candidates for targeted delivery applications, but they could be exploited as tools to deliver siRNAs or regulatory peptides for basic research ${ }^{57}$. Using the same nuclease strategy to remove non-internalizing, cell-surface bound aptamers, the Levy group selected an anti-human transferrin receptor (hTfR) aptamer called $\mathrm{c} 2$ that rapidly internalizes in various $\mathrm{hTfR}-$ expressing tumor cell lines ${ }^{38}$. This cell-internalizing aptamer effectively delivered various molecular cargoes to living cells, such as a small chemotherapeutic drug (e.g., doxorubicin) ${ }^{58}$, therapeutic oligonucleotides (e.g., NF- $\mathrm{kB}$ decoy, and microRNA-126) 58,59 and siRNA-loaded lipid nanoparticles ${ }^{38}$. Unfortunately, c2 competes for the binding of the receptor with its natural ligand, i.e. transferrin (Tf). Although this does not represent a limitation for in vitro applications, the high concentration of circulating $\mathrm{Tf}$ (ranging from 1 to $2.4 \mathrm{mg} / \mathrm{ml}$ ) ${ }^{60}$ is expected to interfere with binding of $\mathrm{c} 2$ to hTfR in vivo. Therefore, Maier et al. recently selected a novel aptamer, named Waz, which recognizes hTfR via a different site than $\mathrm{Tf}$, thus avoiding competition for the receptor binding and potentially signaling a strong potential for the Waz aptamer in in vivo applications ${ }^{39}$. 
A third method to remove cell-surface bound aptamers is to treat the surface of cells with proteinase $\mathrm{K}$ after incubation with aptamers. This treatment leads to the digestion of protein domains exposed on the cell surface, including those targeted by the aptamers. Thus, the RNA ligands that are no longer bound to their cognate epitopes are simply washed off. In a recent paper, the de Franciscis group exploited this approach to select cell-internalizing aptamers that bind insulin receptors to human glioma cells (U87MG cells) ${ }^{49}$.

An important consideration of cell-based selection protocols is that cells having a reduced membrane integrity, such as late apoptotic and necrotic cells, can indiscriminately take up nucleic acids, leading to enrichment of aptamers that non-specifically bind these cells. Partitioning methods that fail to eliminate these populations of cells drastically reduce the success rate of a selection process. To address this, the Mayer group developed what they termed fluorescent-activated cell sorting (FACS)-SELEX ${ }^{44}$. In this strategy, Raddatz et al. first performed a calcein staining of live cells and then used FACS to discriminate between dead and live cells within a CD19+ Burkitt's lymphoma cell sample. Singlechain DNA sequences that were internalized only into the viable cells were recovered and then amplified for more rounds of selection. The authors demonstrated that a DNA aptamer named $\mathrm{C} 10^{44}$ and a truncated form designated $\mathrm{C} 10.36^{45}$ were able to distinguish between Burkitt's lymphoma B cells and primary, non-malignant B cells. Both of these nucleic acid ligands were taken up into Burkitt's lymphoma cells with fast kinetics via clathrin-mediated endocytosis. A combined strategy that utilizes key features of FACSand cell-internalization-SELEX could represent an intriguing strategy of selection because it would simultaneously reduce limitations associated to cell-based SELEX and 
allow for direct monitoring of aptamer enrichment at each round of the selection via a dye-labeled RNA pool.

Certain receptors, such as GPCRs, are internalized only upon binding of their ligands in a process called ligand-induced uptake. Interestingly, targeting different epitopes on the same receptor can affect the route of endocytosis of a defined ligand. For example, Austin et al. demonstrated that an anti-EGFR monoclonal antibody and this receptor's natural ligand, EGF, are taken up by two different mechanisms of endocytosis after binding to distinct epitopes on EGFR ${ }^{61}$. Aptamers that target different sites on a certain receptor could similarly experience different fates upon internalization. With this in mind, Zumrut et al. adopted a variant of cell-internalization SELEX termed LigandGuided Selection (LIGS) to direct aptamers to or away from a known receptor epitope ${ }^{62}$. LIGS exploits natural ligands or other high-affinity reagents such as monoclonal antibodies that recognize the same target receptor to displace and elute specific aptamers. Specifically, when this displacing ligand binds its cognate antigen on a certain cellsurface marker, it outcompetes and displaces aptamer sequences that interact at that site. Zumrut et al. utilized LIGS to select aptamers that bind to CD3 $\varepsilon$-an ectodomain of T-cell Receptor (TCR)-expressed on Jurkat cells ${ }^{62}$. At each round of the selection, target cells were pre-treated with a partially enriched DNA pool that recognized Jurkat cells. An excess of high affinity binding anti-CD3 antibody specific for the CD3 $\varepsilon$ domain was then co-incubated with the same target cells previously loaded with the DNA pool. This allowed displacement of aptamer sequences that bound to the same site of the antibody. Eluted sequences were then recovered and used in the following rounds of selection. In principle, the LIGS strategy could be very useful in directing aptamers to alternative 
epitopes of the same receptor, thereby steering the binding event into different downstream cellular outcomes.

\section{Strategies to Enhance and Monitor Endosomal Escape of Cargoes}

The therapeutic success of a given cell-internalizing platform can be strongly dependent on the ability of that platform to mediate efficient cytosolic translocation of cargoes.

Thus, the efficiency of endosomal escape during a cycle of uptake needs to be improved significantly relative to that estimated for traditional delivery systems (i.e., $<0.01 \%)^{20}$.

Over the past years, several strategies to increase cytoplasmic accessibility of cargoes have been developed that either exploit endosomal destabilizing agents that alter permeability of endosomal membranes, such as chloroquine and other small endosomolytic molecules, or that utilize engineered viral and antimicrobial peptides with membrane-penetration properties ${ }^{63}$. Unfortunately, both approaches suffer from some limitations. When used at the effective concentrations, endosomolytic agents lyse not only endosomes that contain the therapeutic payload, but also a significant fraction of other vesicles including lysosomes ${ }^{20}$. This can lead to the release in the cytoplasm of lytic enzymes, resulting in unacceptable cell toxicity. Engineered variations of cell penetrating peptides (CPPs) (such as truncated HIV-1 TAT peptide or Aurein1,2) containing a few hydrophobic, cationic domains or a combination of them represent promising alternatives that are reported to be non-toxic in several studies ${ }^{64-66}$. However, once therapeutic cargoes are internalized into the endosomes, CPP's can enhance their escape of only five- to eight-fold, which is still not enough to generate effective delivery platforms for clinical applications ${ }^{20}$. The following section highlights strategies and 
technologies that could be adopted into current cell-internalization SELEX protocol to enhance cytosolic translocation properties of aptamers and, consequently, of their cargoes.

\section{Novel Strategies to Enhance Cytosolic Accessibility of Payloads}

A complex system of intracellular vesicles and trafficking is responsible for the fate of an internalized cargo. As shown in Fig 2.5, upon endocytosis, any cargo contained in endocytic vesicles is trafficked into endosomes (early endosomes), from which it can be sorted either back to the surface of the cell (recycling endosomes) or into other compartments (late endosomes) to finally reach the lysosomes where it is at risk of degradation by hydrolytic enzymes. Therefore, a major limitation for an internalized delivery platform occurs when it is sorted in vesicles that are headed to the lysosomal compartment. In fact, when this platform is located in non-recycling endosomes, there is only a narrow window of time that allows escape of cargoes into the cytosol before their degradation.

To increase the efficiency of endosomal escape, emerging strategies aim to delay endosome maturation of non-recycling vesicles, thus increasing retention of cargoes in these vesicles and avoiding fast degradation in lysosomes ${ }^{67}$. For example, the endosomal sorting complex required for transport-I (ESCRT-I) plays a critical role in the maturation and trafficking of endosomal cargoes, and, when ESCRT-I is associated with other accessory proteins, it is involved in the generation of particular late endosomes, i.e. multivesicular bodies (MVBs). By knocking down two components of ESCRT-I (i.e., TSG101 and VPS28), Wagenaar et al. observed that delivered antagomiR able to 
downregulate the oncogenic miRNA-21 displayed increased cytotoxic effect and tumor regression in vivo ${ }^{68}$.

Similarly, Wang et al. used an inhibitor of the Niemann-Pick type C1 (NPC1) protein, termed NP3.47, to delay maturation of non-recycling endosomes such as late endosomes and lysosomes ${ }^{69}$. NPC1 is a late endosomal, membrane-associated protein that regulates cellular cholesterol trafficking pathways ${ }^{70}$. Interestingly, co-treatment of cancer cells with NP3.47 and an siRNA delivered via lipid nanoparticles (LNPs) resulted in an enhancement of both siRNA-mediated gene silencing and retention time in late endosomes/lysosomes as compared with controls in a variety of cell lines ${ }^{69}$, potentially indicating that increasing the residence time of a cargo in non-recycling endosomes can extend the window of time for a more efficient escape into the cytosol of RNA sequences.

The cellular endosomal trafficking machinery is complex. Understanding this complexity and elucidating the roles of its major components could rationally accelerate the development of more efficient strategies for targeted delivery. For instance, as detailed above, altering the function of ESCRT-I or using NPC1 inhibitors demonstrated that effective and careful manipulation of factors involved in the cellular endosomal trafficking machinery led to more efficient and apparently non-toxic endosomal escape of therapeutic RNA cargoes. The possible implications of these results are significant. In theory, delaying maturation of non-recycling vesicles is a strategy that could be adopted in a selection process. In the current cell-internalization SELEX, upon binding to a cellsurface marker, a fraction of the internalized nucleic acid ligands are recycled back to the plasma membrane, and then endocytosed again for a new cycle of 
internalization/recycling. On the other hand, other aptamer sequences are expected to be sorted first into late endosomes and finally to lysosomes, where they are efficiently degraded by lysosomal nucleases, showing an overall poor endosomal escape efficiency. However, the use of a modified selection in which one or more endosomal trafficking/sorting regulators (such as ESCRT-I or NPC1) are targeted to delay endosome maturation could enhance the retention of aptamers in non-recycling vesicles. Extending the window of time before their lysosomal degradation could offer the chance of a more favorable endosomal escape of a significant amount of nucleic acid sequences. An alternative to these strategies could be represented by the conjugation of aptamers to $\mathrm{pH}$-dependent polymeric or peptide molecules that become protonated in acidic compartments. Interestingly, when located in mature endosomes $(\mathrm{pH} \leq 6)$, these molecules can trigger an osmotic swelling of the vesicles (i.e., proton sponge effect), which enhances cargo release into the cytosol. An example of this approach was reported by Liu et al. ${ }^{71}$. These authors conjugated an anti-PSMA aptamer-siRNA chimera via a dsRNA binding domain (dsRBD) to a pH-dependent polyhistidine. The presence of this short histidine oligomer enhanced cytosolic delivery of the aptamer chimera, leading to improved siRNA-mediated silencing of its target gene ${ }^{71}$. Further in vitro and in vivo studies are required to fully assess safety and efficacy of this strategy. An example of an aptamer that can, in some extent, gain cytosolic accessibility is the DNA aptamer (AS1411). This molecule is a G-quadruplex forming oligonucleotide that specifically binds nucleolin, a multifunctional nuclear ribonucleoprotein overexpressed in cancer cells where it shuttles among the nucleus, the cytoplasm, and the cell-surface. AS1411 was originally identified during a screen of antisense oligonucleotides for 
antiproliferative activity toward several human tumor cells ${ }^{72}$. This DNA aptamer is currently undergoing clinical trials as anticancer agent ${ }^{73}$. Interestingly, AS1411 can internalize via macropinocytosis, but a recent work demonstrated that its initial cell uptake is independent of binding to nucleolin ${ }^{74}$. Interaction with this ribonucleoprotein, however, is still essential for the antiproliferative activity of AS1411 and to stimulate further uptake of AS1411 via macropinocytosis ${ }^{75}$. The mechanism by which AS1411 escapes from the macropinosomes/endosomes and accesses the cytoplasm remains unclear, but it is likely that nucleolin's ability to shuttle between different compartments seems to play an essential role. 


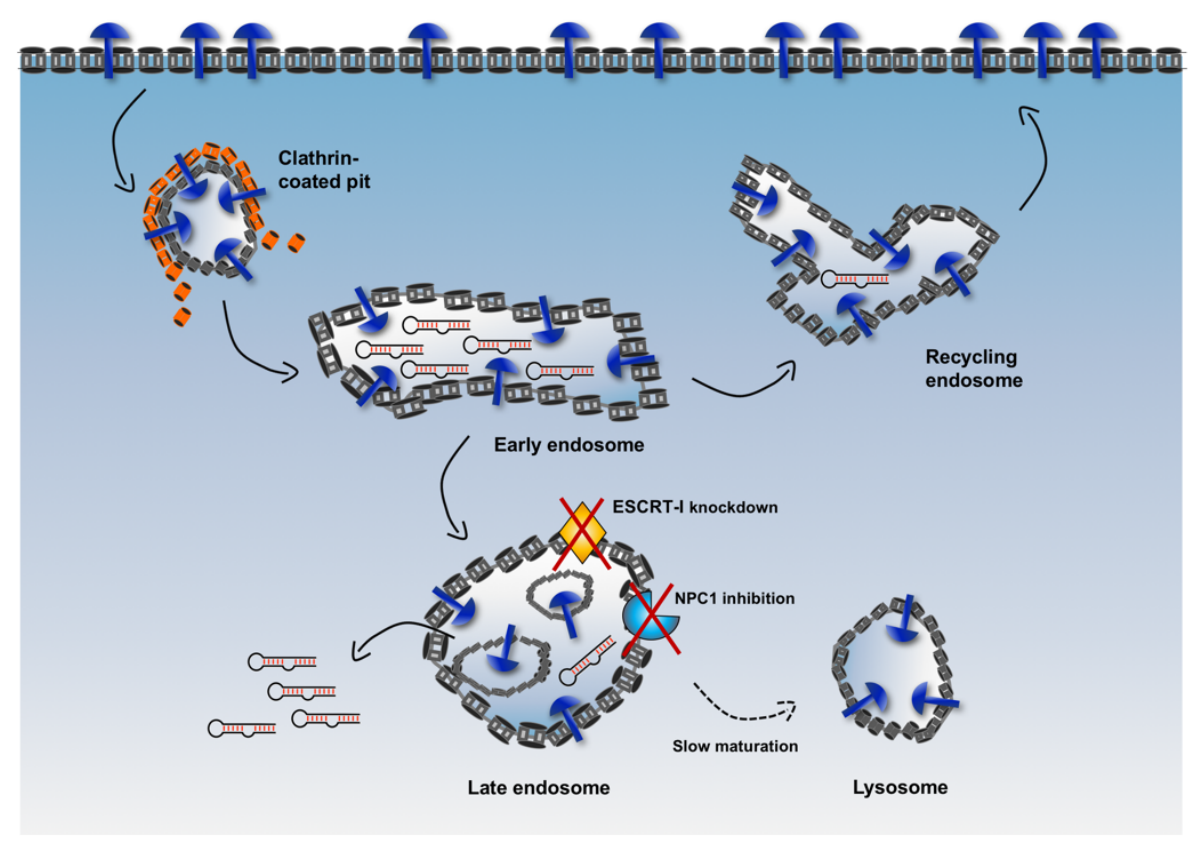

Figure 2.6. Schematic illustration of the expected vesicular trafficking of internalized aptamers and potential strategy to enhance endosomal escape. Upon binding to the target receptor, aptamers enter cells via clathrin-mediated endocytosis (or alternatively via caveolin-dependent uptake) and then are trafficked into early endosomes. In this compartment aptamers can also dissociate from the target receptor. Some receptors (such as transferrin receptor) can be recycled back to the surface, while other (like receptor tyrosine kinases, RTKs) are sorted to the endolysosomal compartment, which results in their downregulation by proteolysis. Internalized aptamers can follow a similar fate. They can be sorted back to the surface via recycling endosomes or into late endosomes. Some innovative strategies aim to delay endosomal maturation and extend the residence time of internalized molecules in non-recycling vesicles (for instance, targeting endosomal-associated proteins, such 
as ESCRT-I and NPC1). This can enhance the overall escape rate during each cycle of endocytosis. 


\section{Traditional and Innovative Methods to Monitor Endosomal Escape of Cargoes}

Generating efficient cytoplasmic delivery platforms requires highly sensitive and robust methods to measure cytosolic penetration and discriminate between internalization and cytosolic accessibility. Improvements upon current methodologies are needed to achieve this distinction and to evaluate aptamer uptake during the SELEX process. Fluorescence microscopy is one of the most used strategies to assess internalization of dye-labeled aptamers in target cells. Standard microscopy techniques enable qualitative analysis of the aptamer-mediated intracellular delivery of molecular payloads, especially when duallabeled nanostructures are used, bearing one fluorophore on the targeting ligand and a different dye on the cargo ${ }^{58,76}$. Upon receptor-mediated or ligand-induced endocytosis, the internalized aptamers and cargoes are located in endocytic vesicles, where their increased local concentration leads to a higher signal-to-noise ratio and punctate appearance. Unfortunately, it is more challenging to discern aptamer fluorescence in the cytoplasm using conventional microscopy settings and instrumentation. Dispersion of nucleic acid molecules into the cytosol decreases overall aptamer concentration and drastically reduces the signal-to-noise ratio. Therefore, novel strategies are needed to address this limitation. This section analyzes current methods used for assessing internalization and endosomal escape of different molecular cargoes. Our goal is to highlight advantages and drawbacks of these methods and identify those that could be incorporated into a SELEX process and be part of the aptamer technology. Intriguing results were recently provided by the Giangrande group. To visualize internalized aptamers, Hernandez et al. developed a method called antibody amplification microscopy ${ }^{77}$. In brief, an RNA aptamer (C4-3) that internalizes into TrkB-expressing 
cells was first conjugated to fluorescein (FAM), but the conjugate gave only a minimal fluorescence upon cell incubation with FAM-C4-3. The authors then performed immunostaining using a primary antibody that recognizes FAM and a dye-labeled secondary antibody labeled with multiple AF488 fluorophores per molecule, thus increasing the overall brightness of this reagent. Antibody-mediated amplification of the fluorescence signal allowed effective detection of FAM-C4-3 inside TrkB-expressing cells. Although this strategy did not discriminate between cytoplasmic and endosomal signal of the aptamers, it suggests that an amplification-based approach could potentially be used to detect aptamers in the cytosol. Variations of this method able to specifically target cytoplasmic RNA molecules might represent a valid alternative for aptamer detection.

Exploiting a novel and highly sensitive fluorescence microscopy technique, a recent study showed release of siRNA delivered via lipoplexes (i.e. lipofectamine-siRNA complexes) or LNPs from individual endosomes ${ }^{21}$. Fluorescence of siRNA molecules was monitored using an imaging system based on multiple focal planes with different exposure times. Long exposure times were used for one of these focal planes to intentionally overexpose bright areas (i.e., endocytic vesicles containing lipoplex-siRNA complexes) and detect the weak fluorescence of free siRNAs translocated in the cytosol. This strategy could potentially have a great impact on understanding and monitoring endosomal escape of cargoes. However, the unconventional microscopy instrumentation is not accessible to the majority of research laboratories, and its success is dependent on the use of sophisticated approaches. Therefore, while it is starting to become possible to discern translocation of cargoes from endosomes to the cytoplasm using fluorescence 
microscopy, additional developments are needed. Moreover, many microscopy strategies are often time consuming especially if several probes or incubation steps are required, as in the case of the antibody amplification microscopy method above. In general, the lowthroughput nature of microscopy does not make this technology the ideal strategy to monitor acquisition of a certain phenotype (such as internalization or cytoplasmic translocation) of a large RNA pool during the course of a SELEX process, where highthroughput systems are preferred.

An attractive high-throughput approach used to assess both binding and internalization properties of fluorescently labeled aptamers is flow cytometry. In this case, to discriminate between surface bound aptamers and internalized species, a stringent, highsalt washing or an enzymatic treatment is required (such as using a cocktail of RNase or Proteinase $\mathrm{K}$ as described above). Therefore, the efficient removal of these surface-bound species plays a critical role in assessing aptamer endocytosis. Although flow cytometry is a high-throughput approach, it suffers from some of the same limitations as fluorescence microscopy, in that this method is not able to discern between cytoplasmic and endosomal localization of aptamers.

The Dehua Pei group offered a potential solution that might overcome the difficulty in monitoring molecules that have escaped from endosomes. In a recent paper, Qian et al. exploited a pH-sensitive fluorophore called naphthofluorescein (NF) coupled to peptide sequences to assess their cytoplasmic translocation ${ }^{78}$. Interestingly, only the deprotonated form of NF is fluorescent ( $\mathrm{pKa}$ of $\mathrm{NF}$ is $\sim 7.8$ ). Therefore, NF is expected to be nearly completely protonated and non-fluorescent inside the acidic endosomes $(\mathrm{pH} \leq$ 6.0), but a fluorescence enhancement could be detected by flow cytometry or 
fluorescence confocal microscopy, indicating cytosolic accessibility (pH 7.4). The Pei lab took advantage of these NF properties to screen and compare endosomal escape efficiencies of several CPPs. First, the authors assessed and compared the internalization properties of every peptide using a $\mathrm{pH}$-insensitive dye (i.e., rhodamine). Then, the endosomal escape of NF-CPP conjugates was monitored by flow cytometry. Notably, only a minimal fluorescence was observed when NF was conjugated to control peptide sequences that are not able to translocate into the cytosol. In contrast, the authors detected significant fluorescence enhancement upon internalization of NF-CPP conjugates. The extent of fluorescence activation was directly correlated with the endosomal escape properties of each peptide sequence. Using this strategy, the authors identified a cyclic peptide (called $\mathrm{cF} \Phi \mathrm{R} 4$ ) that escapes from the endocytic pathway $\sim 5$-fold more efficiently relative to the well-characterized HIV-1 TAT peptide.

Conjugating $\mathrm{pH}$-sensitive fluorophores to known cell-internalizing aptamers could represent a promising and accessible strategy to compare both internalization and cytosolic translocation properties of sequences identified in previous selections. Moreover, a modified cell SELEX that uses a nucleic acid library linked to a $\mathrm{pH}$-sensitive dye could be used in each round of the protocol to monitor cytosolic entry via flow cytometry. This selection approach could be further modified by adopting subcellular fractionation as a partition method to separate aptamer sequences located in different subcellular compartments (such as cytosol and endocytic vesicles). Arguably, highly efficient methods to evaluate productive cytosolic translocation of aptamers and/or their associated cargoes should directly correlate the escape efficiency with a functional readout, such as a biological output that is only activated by internalized 
payloads that effectively access the cytosol. However, the biological activities for most therapeutic cargoes can only be monitored over time using a loss-of-function approach, such as target down-regulation by RNAi, and they often require a time scale (usually $>12 \mathrm{~h}$ ) that is not compatible with a SELEX protocol due to accumulated digestion of internalized aptamers by lysosomal or cytosolic nucleases. Typically, validation of the biological activity of therapeutic cargoes delivered via aptamers can only be evaluated after some rounds or at the end of the selection. In contrast, a different functional readout that exploits rapid gain-of-function assays, such as monitoring increase of fluorescence or luminescence as a consequence of a biological effect, could be more easily incorporated in a SELEX protocol.

Recently, Lonn et al. have developed a fluorescence-based quantitative cytosolic translocation assay to monitor peptide-mediated delivery of cargoes into the cytosol ${ }^{65}$. They reported a split-GFP complementation assay in which a large non-fluorescent fragment of GFP, composed of 10 out to the $11 \beta$-strands of GFP, was expressed in cells. The complementary component (GFP $\beta-11)$ required for the function (i.e. fluorescence) was delivered using cell-penetrating peptides, such as HIV-1 TAT peptide. In this approach, fluorescence can only be observed when the TAT-GFP $\beta-11$ complex is able to escape from endosomes and interact with the non-fluorescent GFP in the cytosol. Those authors employed a set of CPPs reported to have cytosolic translocation properties and successfully restored GFP fluorescence. Importantly, the strength of the GFP fluorescence signal was used to quantitatively assess the endosomal escape abilities of each CPP, thus allowing distinction of these peptides into different classes based on their cytosolic translocation efficiency ${ }^{65}$. Overall, the Split-GFP complementary assay offers a 
sensitive assay monitoring a biological activity (protein-protein interaction) with low to zero false positives when utilized to evaluate endosomal escape properties. However, to implement this strategy in a SELEX method or to assess escape of known cellinternalizing aptamers, conjugation to the GFP $\beta-11$ motif should ensure retaining of aptamer folding and restoring of GFP fluorescence. Therefore, to minimize inter- and intra-molecular interactions, different coupling strategies should be evaluated before starting a selection.

An intriguing alternative to this approach might exploit a split-aptamer system that generates a specific readout only upon formation of a fully functional aptamer structure. One component of this split system could be directly connected to a combinatorial library, and the other component could be expressed in the target cells, such that signal is only observed upon successful delivery of the complementary strand. In this regard, our lab recently introduced a tool to monitor RNA-RNA interaction in bacteria. In brief, taking advantages of the light-up properties of fluorogenic RNA aptamers, we engineered a split version of the broccoli RNA aptamer ${ }^{79}$. Hybridization of two non-fluorescent RNA strands restores Broccoli fluorescence in living cells. Current efforts in our lab to monitor endosomal escape of aptamers are directed to express in mammalian cells one of the two strands of the Split-Broccoli system while delivering its complementary strand using known cell-internalizing aptamers. Compared to the Split-GFP complimentary assay, our approach exploits an "all-RNA system" thus offering significant advantages in terms of ease of design, synthesis, and folding assessment. 


\section{Conclusions}

Over the last decade, aptamer-mediated targeted delivery of therapeutics in malignant cells has emerged as a promising cancer therapy approach with limited off-target effects. Current efforts are directed towards increasing the performance of these therapeutic aptamer nanostructures to facilitate their clinical translation. Particularly, several strategies aim to enhance the cytosolic accessibility of cargoes upon their aptamermediated internalization. Since its discovery, the SELEX process has undergone a continuous evolution. Existing methods have been modified and consequently evolved to meet new biological and technological requirements and ultimately to generate more versatile and potent nucleic acid ligands. In the case of cell targeting aptamers, early SELEX protocols aimed to identify nucleic acid ligands that bind recombinant purified cell-surface proteins. The evolution of this approach led to more complex selection strategies. In the early days, aptamers able to recognize whole tumor cells with high selectivity were generated through an innovative cell SELEX method. Later, cellinternalization SELEX was introduced to select aptamers that not only bind cell-surface markers in their physiological environment but that also internalize into malignant cells. In each of these SELEX variations, additional properties, such as recognition of whole cells instead of recombinant proteins and rapid endocytosis in cancer cells, were included in the selected ligands. However, clinical translation is still limited by escape into the cytosol. Thereby, to overcome current limitations of the aptamer-mediated targeted delivery of therapeutics, a further evolution of SELEX is needed. Innovative selection protocols that incorporate technologies and tools developed for other biomolecules (such as peptides and proteins) could result in the effective identification of nucleic acid ligands 
with extended biological functionalities. New generations of aptamers will go beyond binding and internalization and will be able to perform productive cytosolic translocation of therapeutics, thus accelerating clinical translation of cell targeting aptamer nanostructures. 


\section{Reference:}

(1) Sun, T.; Zhang, Y. S.; Pang, B.; Hyun, D. C.; Yang, M.; Xia, Y. Engineered Nanoparticles for Drug Delivery in Cancer Therapy. Angew. Chem. Int. Ed. Engl. 2014, 53 (46), 12320-12364. https://doi.org/10.1002/anie.201403036.

(2) Srinivasarao, M.; Galliford, C. V.; Low, P. S. Principles in the Design of LigandTargeted Cancer Therapeutics and Imaging Agents. Nat. Rev. Drug Discov. 2015, No. February. https://doi.org/10.1038/nrd4519.

(3) Guidotti, G.; Brambilla, L.; Rossi, D. Cell-Penetrating Peptides: From Basic Research to Clinics. Trends Pharmacol. Sci. 2017, 38 (4), 406-424. https://doi.org/10.1016/j.tips.2017.01.003.

(4) Marqus, S.; Pirogova, E.; Piva, T. J. Evaluation of the Use of Therapeutic Peptides for Cancer Treatment. J. Biomed. Sci. 2017, 24 (1), 21. https://doi.org/10.1186/s12929-017-0328-x.

(5) Khvorova, A.; Watts, J. K. The Chemical Evolution of Oligonucleotide Therapies of Clinical Utility. Nat. Biotechnol. 2017, No. February. https://doi.org/10.1038/nbt.3765.

(6) Juliano, R. L. The Delivery of Therapeutic Oligonucleotides. Nucleic Acids Res. 2016, 44 (14), 6518-6548. https://doi.org/10.1093/nar/gkw236.

(7) Peer, D.; Karp, J. M.; Hong, S.; Farokhzad, O. C.; Margalit, R.; Langer, R. Nanocarriers as an Emerging Platform for Cancer Therapy. Nat. Nanotechnol. 2007, 2 (12), 751-760. https://doi.org/10.1038/nnano.2007.387.

(8) Liu, D.; Auguste, D. T. Cancer Targeted Therapeutics: From Molecules to Drug Delivery Vehicles. J. Control. Release 2015, 219, 632-643. 
https://doi.org/10.1016/j.jconrel.2015.08.041.

(9) Danhier, F.; Feron, O.; Préat, V. To Exploit the Tumor Microenvironment: Passive and Active Tumor Targeting of Nanocarriers for Anti-Cancer Drug Delivery. $J$. Control. Release 2010, 148 (2), 135-146.

https://doi.org/10.1016/j.jconrel.2010.08.027.

(10) Allen, T. M. Ligand-Targeted Therapeutics in Anticancer Therapy. Nat. Rev. Cancer 2002, 2 (10), 750-763. https://doi.org/10.1038/nrc903.

(11) Steichen, S. D.; Caldorera-Moore, M.; Peppas, N. a. A Review of Current Nanoparticle and Targeting Moieties for the Delivery of Cancer Therapeutics. Eur. J. Pharm. Sci. 2012, 48 (3), 416-427. https://doi.org/10.1016/j.ejps.2012.12.006.

(12) Zhou, J.; Rossi, J. J. Cell-Type-Specific, Aptamer-Functionalized Agents for Targeted Disease Therapy. Mol. Ther. Nucleic Acids 2014, 3 (May), e169. https://doi.org/10.1038/mtna.2014.21.

(13) Sanna, V.; Pala, N.; Sechi, M. Targeted Therapy Using Nanotechnology: Focus on Cancer. Int. J. Nanomedicine 2014, 467-483.

(14) Gomes de Castro, M. A.; Höbartner, C.; Opazo, F. Aptamers Provide Superior Stainings of Cellular Receptors Studied under Super-Resolution Microscopy. PLoS One 2017, 12 (2), e0173050. https://doi.org/10.1371/journal.pone.0173050.

(15) Zhou, J.; Rossi, J. Aptamers as Targeted Therapeutics: Current Potential and Challenges. Nat Rev Drug Discov 2016, advance on. https://doi.org/10.1038/nrd.2016.199.

(16) Sun, H.; Zu, Y. Aptamers and Their Applications in Nanomedicine. Small 2015, 1-13. https://doi.org/10.1002/smll.201403073. 
(17) Prakash, J.; Rajamanickam, K. Aptamers and Their Significant Role in Cancer Therapy and Diagnosis. Biomedicines 2015, 3 (3), 248-269.

https://doi.org/10.3390/biomedicines3030248.

(18) Bruno, J. G. A Review of Therapeutic Aptamer Conjugates with Emphasis on New Approaches. Pharmaceuticals. March 19, 2013, pp 340-357.

https://doi.org/10.3390/ph6030340.

(19) Urmann, K.; Modrejewski, J.; Scheper, T.; Walter, J. G. Aptamer-Modified Nanomaterials: Principles and Applications. BioNanoMaterials 2017, 18 (1-2), 117. https://doi.org/10.1515/bnm-2016-0012.

(20) Dowdy, S. F. Overcoming Cellular Barriers for RNA Therapeutics. Nat. Biotechnol. 2017, 35 (3), 222-229. https://doi.org/10.1038/nbt.3802.

(21) Wittrup, A.; Ai, A.; Liu, X.; Hamar, P.; Trifonova, R.; Charisse, K.; Manoharan, M.; Kirchhausen, T.; Lieberman, J. Visualizing Lipid-Formulated SiRNA Release from Endosomes and Target Gene Knockdown. Nat. Biotechnol. 2015, 33 (November 2014), 1-9. https://doi.org/10.1038/nbt.3298.

(22) Dassie, J. P.; Liu, X.-Y.; Thomas, G. S.; Whitaker, R. M.; Thiel, K. W.; Stockdale, K. R.; Meyerholz, D. K.; McCaffrey, A. P.; McNamara, J. O.; Giangrande, P. H. Systemic Administration of Optimized Aptamer-SiRNA Chimeras Promotes Regression of PSMA-Expressing Tumors. Nat. Biotechnol. 2009, 27 (9), 839-849. https://doi.org/10.1038/nbt.1560.

(23) Prakash, T. P.; Graham, M. J.; Yu, J.; Carty, R.; Low, A.; Chappell, A.; Schmidt, K.; Zhao, C.; Aghajan, M.; Murray, H. F.; Riney, S.; Booten, S. L.; Murray, S. F.; Gaus, H.; Crosby, J.; Lima, W. F.; Guo, S.; Monia, B. P.; Swayze, E. E.; Seth, P. 
P. Targeted Delivery of Antisense Oligonucleotides to Hepatocytes Using

Triantennary N-Acetyl Galactosamine Improves Potency 10-Fold in Mice. Nucleic

Acids Res. 2014, 42 (13), 8796-8807. https://doi.org/10.1093/nar/gku531.

(24) Ellington, A. D.; Szostak, J. W. In Vitro Selection of RNA Molecules That Bind Specific Ligands. Nature 1990, 346 (6287), 818-822.

https://doi.org/10.1038/346818a0.

(25) Tuerk, C.; Gold, L. Systematic Evolution of Ligands by Exponential Enrichment: RNA Ligands to Bacteriophage T4 DNA Polymerase. Science 1990, 249 (4968), $505-510$.

(26) McKeague, M.; Derosa, M. C. Challenges and Opportunities for Small Molecule Aptamer Development. J. Nucleic Acids 2012, 2012, 748913. https://doi.org/10.1155/2012/748913.

(27) Dausse, E.; Da Rocha Gomes, S.; Toulmé, J. J. Aptamers: A New Class of Oligonucleotides in the Drug Discovery Pipeline? Current Opinion in Pharmacology. Elsevier October 1, 2009, pp 602-607. https://doi.org/10.1016/j.coph.2009.07.006.

(28) Ng, E. W. M.; Shima, D. T.; Calias, P.; Cunningham, E. T.; Guyer, D. R.; Adamis, A. P. Pegaptanib, a Targeted Anti-VEGF Aptamer for Ocular Vascular Disease. Nat. Rev. Drug Discov. 2006, 5 (2), 123-132. https://doi.org/10.1038/nrd1955.

(29) Vinores, S. a. Pegaptanib in the Treatment of Wet, Age-Related Macular Degeneration. Int. J. Nanomedicine 2006, 1 (3), 263-268.

(30) Lao, Y.-H.; Phua, K. K. L.; Leong, K. W. Aptamer Nanomedicine for Cancer Therapeutics: Barriers and Potential for Translation. ACS Nano 2015, 9 (3), 2235- 
2254. https://doi.org/10.1021/nn507494p.

(31) Maier, K. E.; Levy, M. From Selection Hits to Clinical Leads: Progress in Aptamer Discovery. Mol. Ther. Methods Clin. Dev. 2016, 5 (November 2015), 16014. https://doi.org/10.1038/mtm.2016.14.

(32) Mallikaratchy, P. Evolution of Complex Target SELEX to Identify Aptamers against Mammalian Cell-Surface Antigens. Molecules. MDPI AG February 1, 2017, p 215. https://doi.org/10.3390/molecules22020215.

(33) Marie-Cécile Mercier, Monique Dontenwill, L. C. Selection of Nucleic Acid Aptamers Targeting Tumor Cell-Surface Protein Biomarkers. Cancers (Basel). 2017, 9 (6), 69. https://doi.org/10.3390/cancers9060069.

(34) Li, N.; Nguyen, H. H.; Byrom, M.; Ellington, A. D. Inhibition of Cell Proliferation by an Anti-EGFR Aptamer. PLoS One 2011, 6 (6), e20299. https://doi.org/10.1371/journal.pone.0020299.

(35) Shangguan, D.; Li, Y.; Tang, Z.; Cao, Z. C.; Chen, H. W.; Mallikaratchy, P.; Sefah, K.; Yang, C. J.; Tan, W. Aptamers Evolved from Live Cells as Effective Molecular Probes for Cancer Study. Proc. Natl. Acad. Sci. U. S. A. 2006, 103 (32), 11838-11843. https://doi.org/10.1073/pnas.0602615103.

(36) Somasunderam, A.; Thiviyanathan, V.; Tanaka, T.; Li, X.; Neerathilingam, M.; Lokesh, G. L. R.; Mann, A.; Peng, Y.; Ferrari, M.; Klostergaard, J.; Gorenstein, D. G. Combinatorial Selection of DNA Thioaptamers Targeted to the HA Binding Domain of Human CD44. Biochemistry 2010, 49 (42), 9106-9112. https://doi.org/10.1021/bi1009503.

(37) Volk, D. E.; Lokesh, G. L. R. Development of Phosphorothioate DNA and DNA 
Thioaptamers. Biomedicines 2017, 5 (3), 41.

https://doi.org/10.3390/biomedicines5030041.

(38) Wilner, S. E.; Wengerter, B.; Maier, K.; de Lourdes Borba Magalhães, M.; Del

Amo, D. S.; Pai, S.; Opazo, F.; Rizzoli, S. O.; Yan, A.; Levy, M. An RNA

Alternative to Human Transferrin: A New Tool for Targeting Human Cells. Mol.

Ther. Nucleic Acids 2012, 1 (5), e21. https://doi.org/10.1038/mtna.2012.14.

(39) Maier, K. E.; Jangra, R. K.; Shieh, K. R.; Cureton, D. K.; Xiao, H.; Snapp, E. L.;

Whelan, S. P.; Chandran, K.; Levy, M. A New Transferrin Receptor Aptamer

Inhibits New World Hemorrhagic Fever Mammarenavirus Entry. Mol. Ther.

Nucleic Acids 2016, 5 (5), e321. https://doi.org/10.1038/mtna.2016.32.

(40) Lupold, S. E.; Hicke, B. J.; Lin, Y.; Coffey, D. S. Identification and

Characterization of Nuclease-Stabilized RNA Molecules That Bind Human

Prostate Cancer Cells via the Prostate-Specific Membrane Antigen 1. Cancer Res. 2002, 62, 4029-4033.

(41) Bates, P. J.; Laber, D. A.; Miller, D. M.; Thomas, S. D.; Trent, J. O. Discovery and Development of the G-Rich Oligonucleotide AS1411 as a Novel Treatment for Cancer. Exp. Mol. Pathol. 2009, 86 (3), 151-164.

https://doi.org/10.1016/j.yexmp.2009.01.004.

(42) Soundararajan, S.; Chen, W.; Spicer, E. K.; Courtenay-Luck, N.; Fernandes, D. J. The Nucleolin Targeting Aptamer AS1411 Destabilizes Bcl-2 Messenger RNA in Human Breast Cancer Cells. Cancer Res. 2008, 68 (7), 2358-2365.

https://doi.org/10.1158/0008-5472.CAN-07-5723.

(43) Soundararajan, S.; Wang, L.; Sridharan, V.; Chen, W.; Courtenay-Luck, N.; Jones, 
D.; Spicer, E. K.; Fernandes, D. J. Plasma Membrane Nucleolin Is a Receptor for the Anticancer Aptamer AS1411 in MV4-11 Leukemia Cells. Mol. Pharmacol. 2009, 76 (5), 984-991. https://doi.org/10.1124/mol.109.055947.

(44) Raddatz, M. S. L.; Dolf, A.; Endl, E.; Knolle, P.; Famulok, M.; Mayer, G. Enrichment of Cell-Targeting and Population-Specific Aptamers by FluorescenceActivated Cell Sorting. Angew. Chemie - Int. Ed. 2008, 47 (28), 5190-5193. https://doi.org/10.1002/anie.200800216.

(45) Opazo, F.; Eiden, L.; Hansen, L.; Rohrbach, F.; Wengel, J.; Kjems, J.; Mayer, G. Modular Assembly of Cell-Targeting Devices Based on an Uncommon GQuadruplex Aptamer. Mol. Ther. - Nucleic Acids 2015, 4, e251. https://doi.org/10.1038/mtna.2015.25.

(46) Thiel, K. W.; Hernandez, L. I.; Dassie, J. P.; Thiel, W. H.; Liu, X.; Stockdale, K. R.; Rothman, A. M.; Hernandez, F. J.; McNamara, J. O.; Giangrande, P. H.; Giangrande, P. H. Delivery of Chemo-Sensitizing SiRNAs to HER2+-Breast Cancer Cells Using RNA Aptamers. Nucleic Acids Res. 2012, 40 (13), 6319-6337. https://doi.org/10.1093/nar/gks294.

(47) Chen, C. B.; Dellamaggiore, K. R.; Ouellette, C. P.; Sedano, C. D.; Lizadjohry, M.; Chernis, G. A.; Gonzales, M.; Baltasar, F. E.; Fan, A. L.; Myerowitz, R.; Neufeld, E. F. Aptamer-Based Endocytosis of a Lysosomal Enzyme. Proc. Natl. Acad. Sci. U. S. A. 2008, 105 (41), 15908-15913. https://doi.org/10.1073/pnas.0808360105.

(48) Porciani, D.; Signore, G.; Marchetti, L.; Mereghetti, P.; Nifosì, R.; Beltram, F. Two Interconvertible Folds Modulate the Activity of a DNA Aptamer Against 
Transferrin Receptor. Mol. Ther. Acids 2014, 3 (1), e144.

https://doi.org/10.1038/mtna.2013.71.

(49) Iaboni, M.; Fontanella, R.; Rienzo, A.; Capuozzo, M.; Nuzzo, S.; Santamaria, G.; Catuogno, S.; Condorelli, G.; de Franciscis, V.; Esposito, C. L. Targeting Insulin Receptor with a Novel Internalizing Aptamer. Mol. Ther. Acids 2016, 5 (9), e365. https://doi.org/10.1038/mtna.2016.73.

(50) Ozer, A.; Pagano, J. M.; Lis, J. T. New Technologies Provide Quantum Changes in the Scale, Speed, and Success of SELEX Methods and Aptamer Characterization. Molecular Therapy - Nucleic Acids. Nature Publishing Group August 5, 2014, p e183. https://doi.org/10.1038/mtna.2014.34.

(51) Baneyx, F.; Mujacic, M. Recombinant Protein Folding and Misfolding in Escherichia Coli. Nat.Biotechnol. 2004, 22 (11), 1399-1408. https://doi.org/10.1038/nbt1029.

(52) Sørensen, H. P.; Mortensen, K. K. Soluble Expression of Recombinant Proteins in the Cytoplasm of Escherichia Coli. Microb. Cell Fact. 2005, 4 (1), 1. https://doi.org/10.1186/1475-2859-4-1.

(53) Liu, Y.; Kuan, C. T.; Mi, J.; Zhang, X.; Clary, B. M.; Bigner, D. D.; Sullenger, B. A. Aptamers Selected against the Unglycosylated EGFRvIII Ectodomain and Delivered Intracellularly Reduce Membrane-Bound EGFRvIII and Induce Apoptosis. Biol. Chem. 2009, 390 (2), 137-144. https://doi.org/10.1515/BC.2009.022.

(54) Vinkenborg, J. L.; Mayer, G.; Famulok, M. Aptamer-Based Affinity Labeling of Proteins. Angew. Chem. Int. Ed. Engl. 2012, 51 (36), 9176-9180. 
https://doi.org/10.1002/anie.201204174.

(55) Kruspe, S.; Giangrande, P. Aptamer-SiRNA Chimeras: Discovery, Progress, and Future Prospects. Biomedicines 2017, 5 (3), 45.

https://doi.org/10.3390/biomedicines5030045.

(56) Magalhães, M. L.; Byrom, M.; Yan, A.; Kelly, L.; Li, N.; Furtado, R.; Palliser, D.; Ellington, A. D.; Levy, M. A General RNA Motif for Cellular Transfection. Mol. Ther. 2012, 20 (3), 616-624. https://doi.org/10.1038/mt.2011.277.

(57) Burke, D. H. Cell-Penetrating RNAs: New Keys to the Castle. Mol. Ther. 2012, 20 (2), 251-253. https://doi.org/10.1038/mt.2011.306.

(58) Porciani, D.; Tedeschi, L.; Marchetti, L.; Citti, L.; Piazza, V.; Beltram, F.; Signore, G. Aptamer-Mediated Codelivery of Doxorubicin and NF-?B Decoy Enhances Chemosensitivity of Pancreatic Tumor Cells. Mol. Ther. - Nucleic Acids 2015, 4, e235. https://doi.org/10.1038/mtna.2015.9.

(59) Rohde, J.-H.; Weigand, J. E.; Suess, B.; Dimmeler, S. A Universal Aptamer Chimera for the Delivery of Functional MicroRNA-126. Nucleic Acid Ther. 2015, 25 (3), 141-151. https://doi.org/10.1089/nat.2014.0501.

(60) Mcknight, G. S.; Lee, O. D. C.; Hemmaplardh, D.; Finch, C. A.; Richard, D. Transferrin Gene Expression. 1980, 255 (1), 144-147.

(61) Austin, C. D., De Mazière AM, Pisacane PI, van Dijk SM, Eigenbrot C, Sliwkowski MX, Klumperman J, S. R. Endocytosis and Sorting of ErbB2 and the Site of Action of Cancer Therapeutics Trastuzumab and Geldanamycin. Mol. Biol. Cell 2004, 15 (12), 5268-5282. https://doi.org/10.1091/mbc.E04-07-0591.

(62) Zumrut, H. E.; Ara, M. N.; Maio, G. E.; Van, N. A.; Batool, S.; Mallikaratchy, P. 
R. Ligand-Guided Selection of Aptamers against T-Cell Receptor-Cluster of Differentiation 3 (TCR-CD3) Expressed on Jurkat.E6 Cells. Anal. Biochem. 2016, 512, 1-7. https://doi.org/10.1016/j.ab.2016.08.007.

(63) Erazo-Oliveras, A.; Muthukrishnan, N.; Baker, R.; Wang, T. Y.; Pellois, J. P. Improving the Endosomal Escape of Cell-Penetrating Peptides and Their Cargos: Strategies and Challenges. Pharmaceuticals 2012, 5 (11), 1177-1209. https://doi.org/10.3390/ph5111177.

(64) Li, M.; Tao, Y.; Shu, Y.; LaRochelle, J. R.; Steinauer, A.; Thompson, D.; Schepartz, A.; Chen, Z. Y.; Liu, D. R. Discovery and Characterization of a Peptide That Enhances Endosomal Escape of Delivered Proteins in Vitro and in Vivo. $J$. Am. Chem. Soc. 2015, 137 (44), 14084-14093. https://doi.org/10.1021/jacs.5b05694.

(65) Lönn, P.; Kacsinta, A. D.; Cui, X.-S.; Hamil, A. S.; Kaulich, M.; Gogoi, K.; Dowdy, S. F. Enhancing Endosomal Escape for Intracellular Delivery of Macromolecular Biologic Therapeutics. Sci. Rep. 2016, 6 (1), 32301. https://doi.org/10.1038/srep32301.

(66) Salomone, F.; Cardarelli, F.; Di Luca, M.; Boccardi, C.; Nifosì, R.; Bardi, G.; Di Bari, L.; Serresi, M.; Beltram, F. A Novel Chimeric Cell-Penetrating Peptide with Membrane-Disruptive Properties for Efficient Endosomal Escape. J. Control. Release 2012, 163 (3), 293-303. https://doi.org/10.1016/j.jconrel.2012.09.019.

(67) Sahay, G.; Querbes, W.; Alabi, C.; Eltoukhy, A.; Sarkar, S.; Zurenko, C.; Karagiannis, E.; Love, K.; Chen, D.; Zoncu, R.; Buganim, Y.; Schroeder, A.; Langer, R.; Anderson, D. G. Efficiency of SiRNA Delivery by Lipid Nanoparticles 
Is Limited by Endocytic Recycling. Nat. Biotechnol. 2013, 31 (7), 653-658. https://doi.org/10.1038/nbt.2614.

(68) Wagenaar, T. R.; Tolstykh, T.; Shi, C.; Jiang, L.; Zhang, J.; Li, Z.; Yu, Q.; Qu, H.; Sun, F.; Cao, H.; Pollard, J.; Dai, S.; Gao, Q.; Zhang, B.; Arlt, H.; Cindhuchao, M.; Hoffmann, D.; Light, M.; Jensen, K.; Hopke, J.; Newcombe, R.; GarciaEcheverria, C.; Winter, C.; Zabludoff, S.; Wiederschain, D. Identification of the Endosomal Sorting Complex Required for Transport-I (ESCRT-I) as an Important Modulator of Anti-MiR Uptake by Cancer Cells. Nucleic Acids Res. 2015, 43 (2), 1204-1215. https://doi.org/10.1093/nar/gku1367.

(69) Wang, H.; Tam, Y. Y. C.; Chen, S.; Zaifman, J.; van der Meel, R.; Ciufolini, M. A.; Cullis, P. R. The Niemann-Pick C1 Inhibitor NP3.47 Enhances Gene Silencing Potency of Lipid Nanoparticles Containing SiRNA. Mol. Ther. 2016, 24 (12), 2100-2108. https://doi.org/10.1038/mt.2016.179.

(70) Cruz, J. C.; Sugii, S.; Yu, C.; Chang, T. Y. Role of Niemann-Pick Type C1 Protein in Intracellular Trafficking of Low Density Lipoprotein-Derived Cholesterol. $J$. Biol. Chem. 2000, 275 (6), 4013-4021. https://doi.org/10.1074/jbc.275.6.4013.

(71) Liu, H. Y.; Gao, X. A Universal Protein Tag for Delivery of SiRNA-Aptamer Chimeras. Sci. Rep. 2013, 3, 3129. https://doi.org/10.1038/srep03129.

(72) Bates, P. J.; Kahlon, J. B.; Thomas, S. D.; Trent, J. O.; Miller, D. M. Antiproliferative Activity of G-Rich Oligonucleotides Correlates with Protein Binding. J. Biol. Chem. 1999, 274 (37), 26369-26377. https://doi.org/10.1074/jbc.274.37.26369.

(73) Rosenberg, J. E.; Bambury, R. M.; Van Allen, E. M.; Drabkin, H. a; Lara, P. N.; 
Harzstark, A. L.; Wagle, N.; Figlin, R. a; Smith, G. W.; Garraway, L. a; Choueiri, T.; Erlandsson, F.; Laber, D. a. A Phase II Trial of AS1411 (a Novel NucleolinTargeted DNA Aptamer) in Metastatic Renal Cell Carcinoma. Invest. New Drugs 2014, 32 (1), 178-187. https://doi.org/10.1007/s10637-013-0045-6.

(74) Reyes-Reyes, E. M.; Teng, Y.; Bates, P. J. A New Paradigm for Aptamer Therapeutic AS1411 Action: Uptake by Macropinocytosis and Its Stimulation by a Nucleolin-Dependent Mechanism. Cancer Res. 2010, 70 (21), 8617-8629. https://doi.org/10.1158/0008-5472.CAN-10-0920.

(75) Reyes-Reyes, E. M.; Šalipur, F. R.; Shams, M.; Forsthoefel, M. K.; Bates, P. J. Mechanistic Studies of Anticancer Aptamer AS1411 Reveal a Novel Role for Nucleolin in Regulating Rac1 Activation. Mol. Oncol. 2015, 9 (7), 1392-1405. https://doi.org/10.1016/j.molonc.2015.03.012.

(76) Boyacioglu, O.; Stuart, C. H.; Kulik, G.; Gmeiner, W. H. Dimeric DNA Aptamer Complexes for High-Capacity-Targeted Drug Delivery Using PH-Sensitive Covalent Linkages. Mol. Ther. - Nucleic Acids 2013, 2 (7), e107. https://doi.org/10.1038/mtna.2013.37.

(77) Hernandez, L. I.; Flenker, K. S.; Hernandez, F. J.; Klingelhutz, A. J.; McNamara, J. O.; Giangrande, P. H. Methods for Evaluating Cell-Specific, Cell-Internalizing RNA Aptamers. Pharmaceuticals (Basel). 2013, 6 (3), 295-319. https://doi.org/10.3390/ph6030295.

(78) Qian, Z.; Dougherty, P. G.; Pei, D. Monitoring the Cytosolic Entry of CellPenetrating Peptides Using a PH-Sensitive Fluorophore. Chem. Commun. 2015, 51 (11), 2162-2165. https://doi.org/10.1039/C4CC09441G. 
(79) Alam, K. K.; Tawiah, K. D.; Lichte, M. F.; Porciani, D.; Burke, D. H. A Fluorescent Split Aptamer for Visualizing RNA-RNA Assembly In Vivo. ACS Synth. Biol. 2017, acssynbio.7b00059. https://doi.org/10.1021/acssynbio.7b00059. 


\title{
Chapter 3: Improved virus purification and surface biotinylation of highly lytic enveloped viruses
}

This work will constitute most of the material that will be submitted for publication after further developments outlined at the end of the chapter. Anticipated co-authors include Kwaku D. Tawiah, Amanda Paz, David Porciani, Tommi A. White and Donald H. Burke

\begin{abstract}
The use of virus particles as aptamer selection targets offers tremendous advantages because they can display target proteins in their native contexts. However, highly lytic viruses such as vesicular stomatitis virus (VSV), shred cells during virus propagation, resulting in high levels of cellular debris (non-target contaminants) in crude virus preps that can decrease the performance of SELEX. Prior work outlined a two-step purification process for VSV that involved a pelleting step and buoyant density ultracentrifugation. The pelleting step in this approach resulted in high levels of damaged virus particles. In this work, we developed improved methods for purifying VSV particles and chimeric VSV particles that display filoviral GP in place of VSV-G. We modified the reported method by pelleting the virus particles through a cushion and extending the buoyant density ultracentrifugation times. The effectiveness of these methods in removing cellular debris and other contaminants from the crude preps was evaluated by assessing virus prep quality through electron microscopy, and virus integrity was evaluated through plaque assays. The resulting chimeric virus particles were shown to be suitable for chemical
\end{abstract}


conjugation to biotin and immobilization onto streptavidin-coated (SA-coated) bead surfaces. 


\section{Graphical abstract}

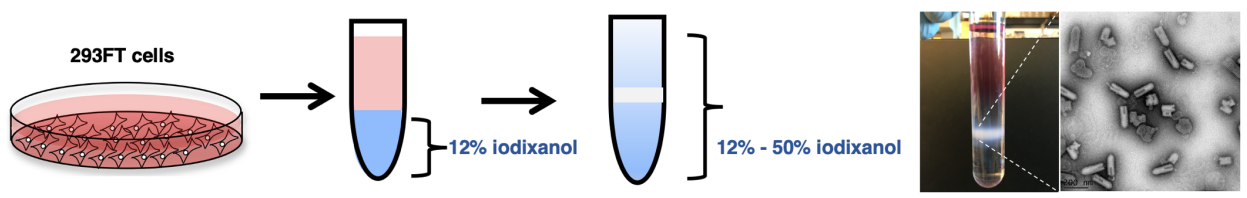




\section{Introduction}

'Virus SELEX' uses whole viruses as selection targets and can generate aptamers with affinity for unique proteins displayed on complex virus surfaces. Virus SELEX is an iterative process that involves three connected steps: The first step involves the incubation of a library with the virus target. This is followed by a partitioning step, where target binders are separated from non-binders. The final step involves the amplification of the target binders utilizing PCR. Binding specificity of the evolving population can be further manipulated through a subtractive step during the selection process that deplete non-specific binders by isolating sequences that bind non-targets molecule such related molecules or the partition platform.

Successful selection of aptamers depends on the consistent presentation of targeted epitopes from one round of selection to the next. When whole viruses are used as the selection target, the quality and integrity of the virus preparation (prep) play significant roles in the success of aptamer discovery. Sequence enrichment during aptamer selections depends on several factors, including low background binding, low off-target binding, and optimal target concentration and purity. Crude virus preps increase off-target binding that decrease the specificity of the resulting aptamer populations or that evolve the library to bind to non-target contaminants in the prep. In contrast, pure virus preps ensure a high level of consistency in epitope (target) presentation in every round of the selection, which can improve the efficiency of the SELEX process. 
Our various aptamer selection platforms make extensive use of chimeric vesicular stomatitis virus that displays filovirus glycoproteins in place of VSV-G $\left(\mathrm{rVSV} / M A R V \_G P \& \mathrm{rVSV} / \mathrm{MARV} \text { GP }\right)^{2-4}$. The virus particles are replicationcompetent and highly lytic, resulting in a huge amount of cellular debris during virus propagation. Filoviruses are highly virulent viruses that, thus research done on filoviruses require high level containment facilities (Biosafety level 4, BSL4). Such facilities are limited therefore, chimeric virus particles serve as excellent BSL2 surrogates. Having replication competent surrogates reduces the batch-to-batch variability that is associated with other surrogates such pseudotyped. Virus pseudotyping requires transfection if many components, which can be a source of variation from batchto-batch due to transfection efficiency. Crude preps of these lytic virus particles have significant levels of contaminants and can be expected to lead to extensive off-target binding by the evolving aptamer population. Such high levels of contaminants can limit the enrichment of target specific sequences when the crude virus preps are used as a selection target ${ }^{1}$. Therefore, pure virus preps are essential for more efficient virus SELEX. Here, we describe the propagation, purification, and surface biotinylation of highly lytic chimeric particles (rVSV/EBOV_GP and rVSV/MARV_GP). The methods outlined here were adapted for preparing virus particles that were used as selection targets in a "virus SELEX" chapters 4 \& 5. 


\section{Results and discussion}

Propagation of chimeric virus particles

Filoviruses are highly pathogenic to humans, and research work performed on them requires a biosafety level 4 (BSL4) laboratory. BSL4 labs are expensive to operate and there are limited numbers of them worldwide. As a solution to these limitations, many researchers utilize surrogate pathogen models such as pseudotyped viruses, chimeric viruses, and virus-like particles that can be safely handled in BSL2 labs. As the first step towards developing aptamers that bind to filovirus surfaces, the project utilized chimeric vesicular stomatitis particles that display filovirus glycoproteins (GP) (rVSV/EBOV_GP and rVSV/MARV_GP, both of which can be handled under BSL2 conditions) as surrogate targets in place BSL4 filoviruses for the selection.

Both rVSV/EBOV_GP and rVSV/MARV_GP viruses were propagated in HEK293FT cells because HEK293FT-cells are highly susceptible to VSV and can produce high titers. To generate high titers with minimal amounts of cellular debris and defective particles, confluent HEK293FT cells were infected with chimeric viral particles at a low multiplicity of infection (MOI 0.01). Initial infections performed at a higher MOI $(>0.1)$ resulted in low virus titers due to severe cell-lytic events occurring in a short time frame. The virus supernatant was harvested between $12-16 \mathrm{hr}$ post-infection ( $\sim 50 \%$ cytopathic effects) to minimize the higher cell lysis events associated with later time points. High cell lysis at later times increases cellular debris in the supernatant with minimal return in virus titer. 


\section{Three-step purification generates cleaner prep than two-step purification}

\section{Overview of the purification process}

The virus supernatant was concentrated and purified using a density gradient ultracentrifugation process adapted from Diallo et $a l^{5}$. with some modifications. First, the pelleting step was performed through an iodixanol cushion and the pellet was slowly resuspended over a longer period of time to minimize the number of broken virus particles. Also, the density gradient ultracentrifugation time was extended from $1 \mathrm{hr}$ to 12 hrs. Overall, the modified purification process involves three steps. Step one involves filtration to eliminate larger cellular debris. Step two involves the concentration and partial purification through an iodixanol cushion. Step 3 involves a more extensive purification using a density gradient ultracentrifugation (Fig 3.1). Steps two and three utilized iodixanol (Optiprep) as a separation media because previous work reported that it is clean, inert, and less harsh on envelope viruses. These observations were consistent with a previous report that compared the distribution of intact virus particles and the bioactivity of adeno associated virus (AAV) particles purified with iodixanol and other density gradient media such as cesium chloride and sucrose $\mathrm{e}^{6,7}$. In our hands, a combination of these three purification steps consistently generated relatively clean preps of chimeric particles. The methods developed in this chapter were used in the purification of the virus particles that served as selection targets for the new aptamers (chapters 4 and 5) and for other applications. 


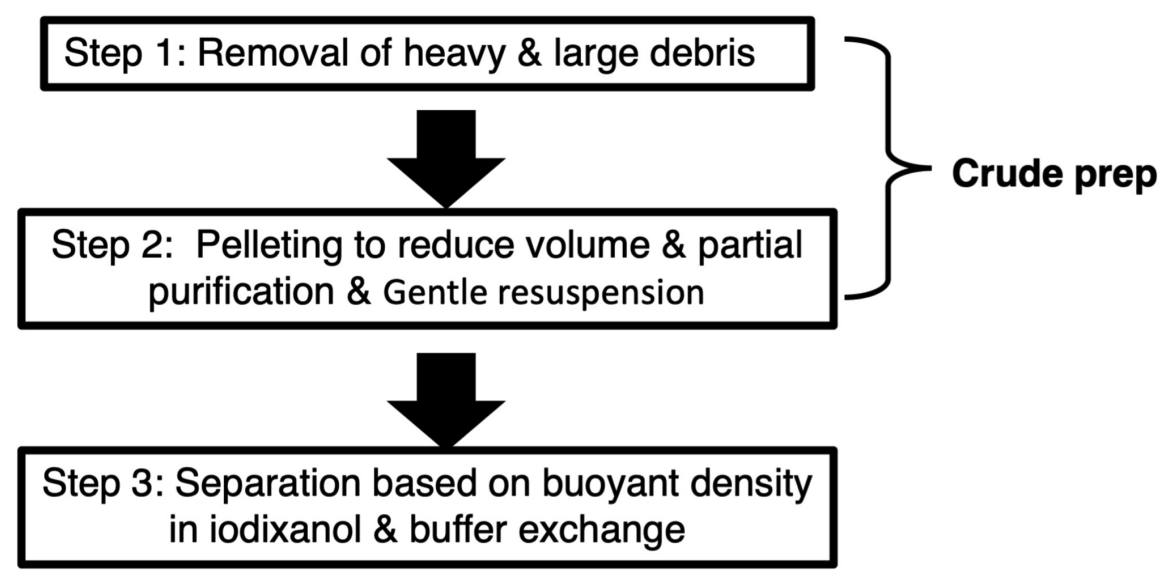

Figure 3.1. Overview of the virus purification process. First two steps remove heavier and larger debris and reduce virus sample volume to manageable volumes. The last step separates virus particles from other contaminants based on the differences in their buoyant densities in iodixanol. 


\section{Step 1}

The first step in the purification was designed to eliminate larger cell fragments in the harvested supernatant. The virus supernatant was spun to eliminate heavy components, such as whole cells. To further exclude larger particulates, the resulting supernatant after the centrifugation was filtered through a $0.2 \mu \mathrm{m}$ polyethersulfone (PES) membrane filter. The crude filtrate can be used for infection of fresh cells, further purified as below, or stored at $-80^{\circ} \mathrm{C}$ for future use.

Step 2

The second step in the purification partially purifies the filtrate from step 1 and reduces it to manageable volumes. In this step, the filtrate was layered onto a $12 \%$ iodixanol cushion and spun using a fixed angle rotor. Following centrifugation, the virus pellet was resuspended in $2 \mathrm{ml}$ phosphate-buffered saline by gently rocking the pellet in buffer overnight at $4^{\circ} \mathrm{C}$ (about 50- fold volume reduction). Plaque assays confirmed the presence of infectious virus particles. The steps described thus far were used in the purification of virus particles that served as targets for several unsuccessful SELEX attempts (appendix 1). Subsequent negative stain electron microscopy (EM) of this virus prep revealed the presence of virus particles but also high levels of contaminants (Fig 3.2). With so much opportunity for off-target binding, the subtractive steps of the selection rounds were not sufficient to remove nucleic acid species with affinity for unwanted targets to generate target-specific populations. Therefore, a third step was included to further purify virus particles. 


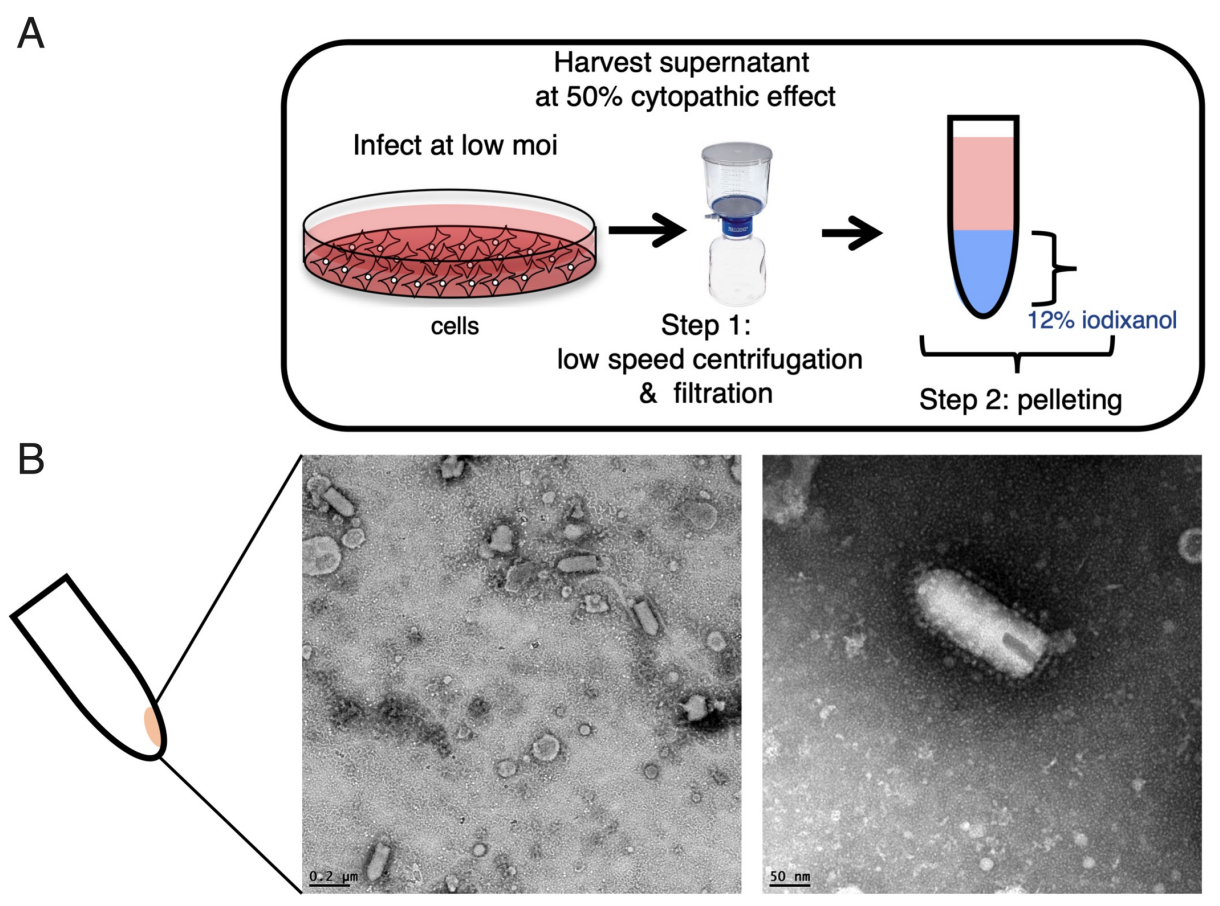

Figure 3.2. Overview and evaluation of the two-step virus purification process. A. To generate virus particles to be used as target for in vitro selection, confluent HEK293 FT cell were infected at a low MOI. The virus supernatant was harvested $16 \mathrm{hr}$. post infection, filtered, and pelleted through iodixanol cushion. B. Virus particles prep quality up to this point of the purification was analyzed using electron microscopy 


\section{Step 3}

The final step separates virus particles from contaminants based on differences in buoyant density in an iodixanol gradient. In this step, the virus suspension from the previous step was layered onto a $12 \%-35 \%$ continuous iodixanol gradient and subjected to ultracentrifugation. We isolated a cloudy band (virus particles) observed at about halfway in the gradient (Fig 3.3A) and performed a buffer exchange by filtration through a molecular weight cutoff membrane. The buffer exchange included three wash steps with $2 \mathrm{~mL}$ PBS. Virus samples were aliquoted and stored at $-80^{\circ} \mathrm{C}$. Virus prep quality and particle integrity were analyzed through EM. The prep from three-step process was cleaner than the prep from the two-step process (Fig 3.3B), suggesting that the density gradient step removes most of the contaminants. However, we consistently observed broken particles in our virus preps and structures that resemble the virus genome coated in nucleocapsid protein (Fig 3.3B). 


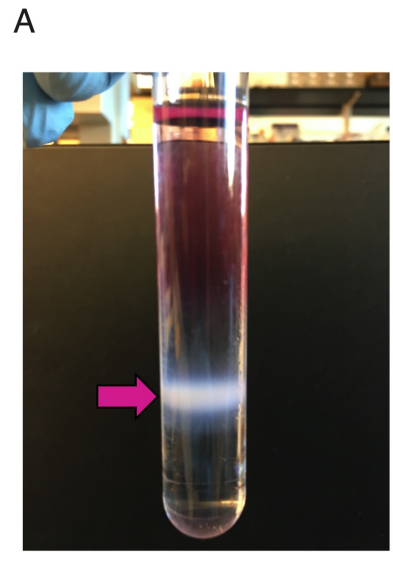

B

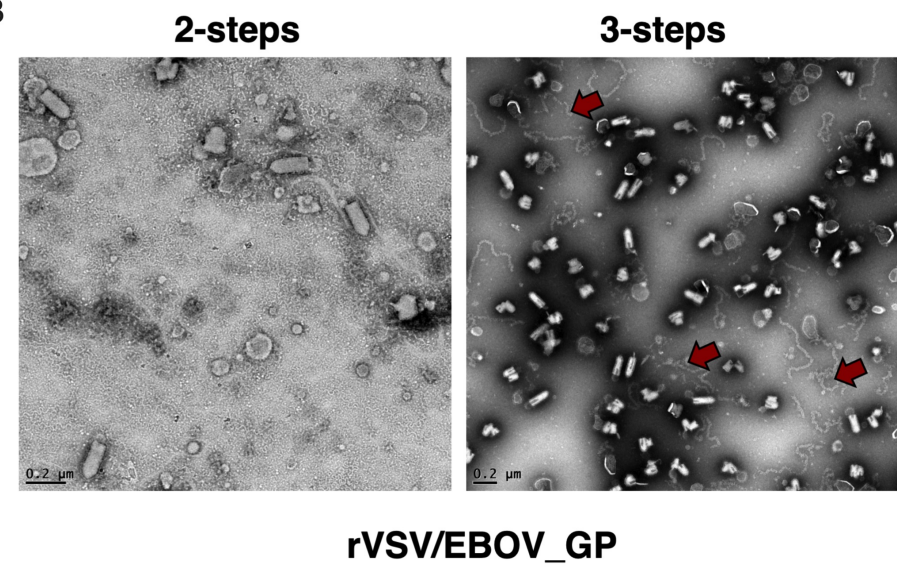

Figure 3.3. Image of cloudy band and EM images from 2-step and 3-step preps A. Image of virus cloudy band (pink arrow) following ultracentrifugation through an iodixanol gradient. B. EM analysis and comparison of virus prep quality resulting from the two-step purification process and the three-step process. The three-step process generated a relatively clean virus prep compared to the 2-step process that has high level of contaminants. There is still debris that appeared to be virus genome (red arrows) in the cleaner prep from the three-step process. 


\section{Examining potential cause of fragmented virus particles in the virus}

\section{preps}

Enveloped viruses are fragile and can easily be ruptured by several factors before or during the purification process described in the previous sections. We speculated that the broken virus particles we observed in the electron micrographs are either a result of incomplete virus maturation and budding or result from disruption during buffer exchange at the end of the purification. These two possibilities were examined to determine the source of the damaged virus particles observed in the electron micrographs. First, if the fragmented particles are a consequence of incomplete virus maturation and budding, then longer centrifugation times should separate the intact particles from fragmented ones, based on the difference in densities. Several ultracentrifugation times were evaluated $(2,6 \& 12 \mathrm{hr}$.) to isolate intact particles. EM analysis of virus particles shows that more prolonged centrifugation times do not decrease the proportions of broken virus particles (Fig 3.4). Next, we recovered the virus band in three fractions (top, middle, and bottom) after $12 \mathrm{hr}$. of ultracentrifugation in the iodixanol gradient and evaluated the level of fragmented particles in all the fractions evaluated by EM. The micrographs showed a relatively high proportion of short particles in the top fraction compared to the middle and bottom fractions. However, all the fractions show some broken particles and what appeared to be nucleocapsid-coated virus genome in their corresponding micrographs (Fig 3.5). These results suggest that some steps after the density gradient ultracentrifugation step might be fragmenting the particles. Another explanation could be that the gradient (12\%-35\%) is not sufficient to separate intact particles from broken ones. 


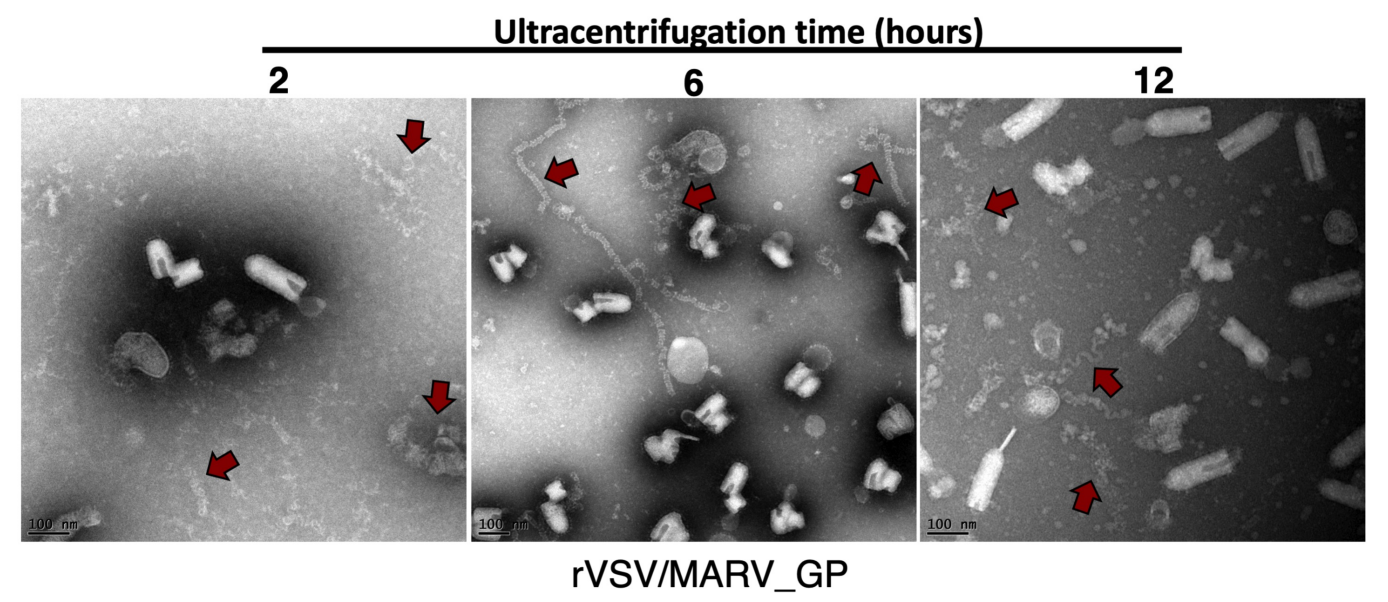

Figure 3.4. Negative stain EM analysis of virus prep from different ultracentrifugation times. Ribonucleoproteins (red arrows) are present in all samples regardless of the centrifugation time. 

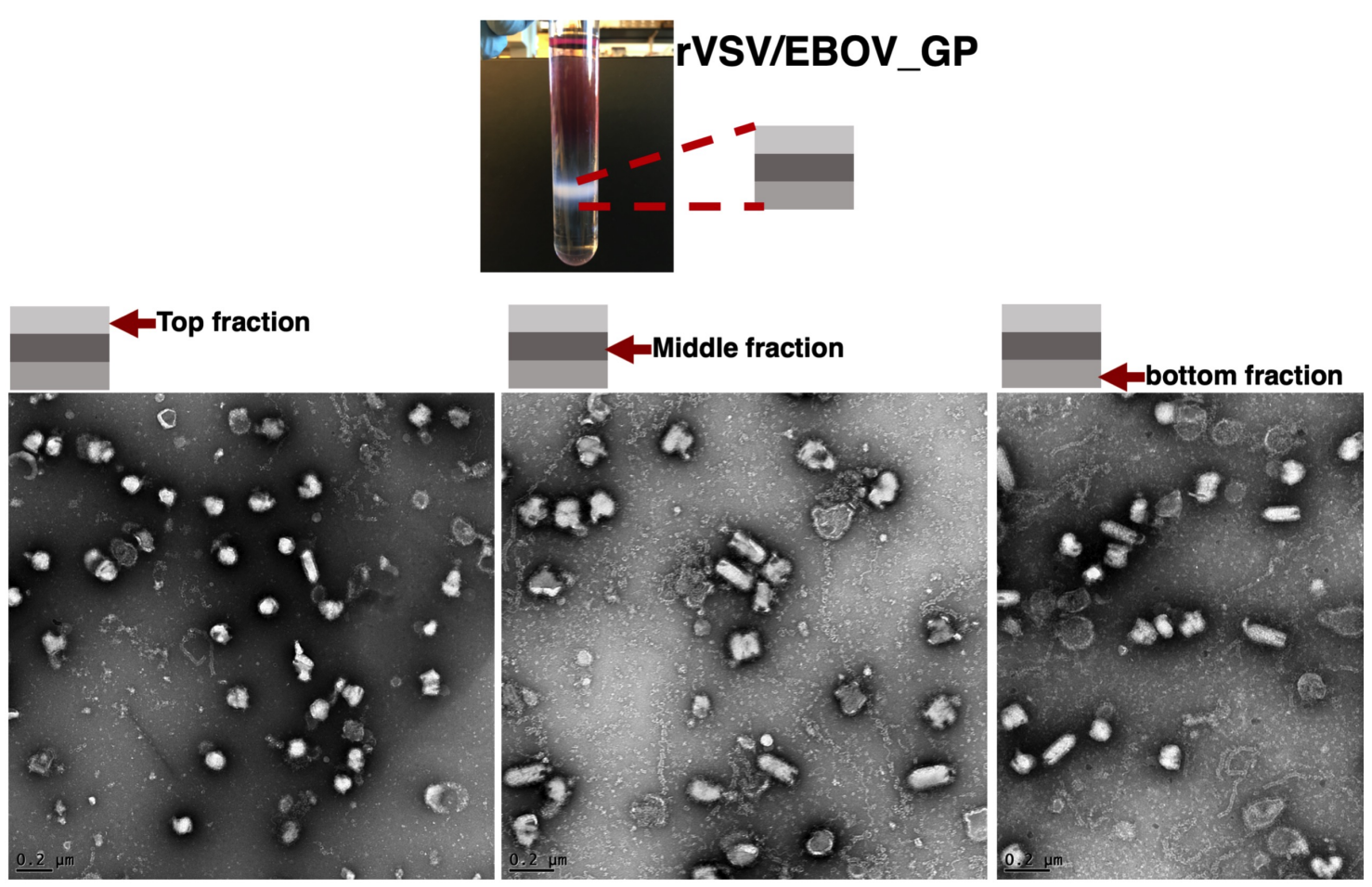

Figure 3.5. EM evaluation of fractionated virus bands following ultracentrifugation of the virus samples through iodixanol gradient. The virus band observed was collected in fractions (top, middle and bottom). Samples from these fractions were analyzed by negative stain electron microscopy. Ribonucleoproteins are present in all the fractions. 
The only step after the density-gradient ultracentrifugation is buffer exchange through a molecular weight cut-off membrane under low-speed centrifugation. To evaluate the potential impact of the buffer exchange step on the virus particle integrity, the filtration step described was substituted with overnight dialysis. Again, EM imaging of virus particles after dialysis showed no noticeable difference in the proportion of broken particles compared to particles that went through the filtration step (Fig 3.6). Lastly, the virus particles were treated with a cocktail of potent RNases in an attempt to degrade what appeared to be virus genomes. Again, there was no discernable depletion of the genome-like contaminants in the samples (Fig 3.7). 


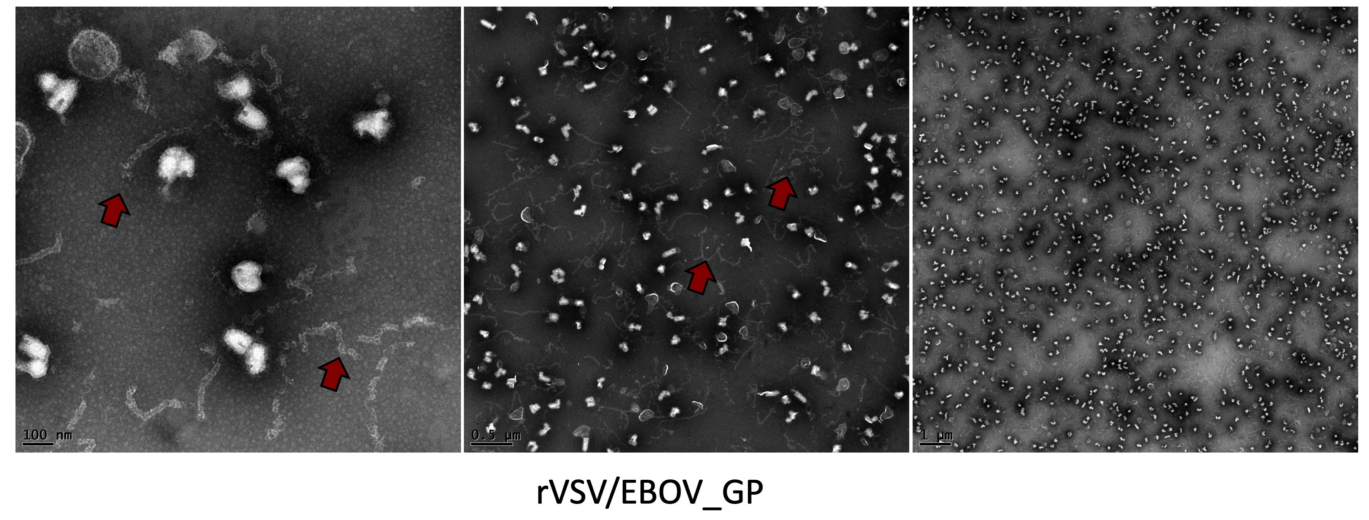

Figure 3.6. Negative stain EM analysis of virus prep following overnight dialysis show the presence of what structures that appears to be ribonucleoprotein complex (red arrow). Micrographs were taken at different magnifications. 


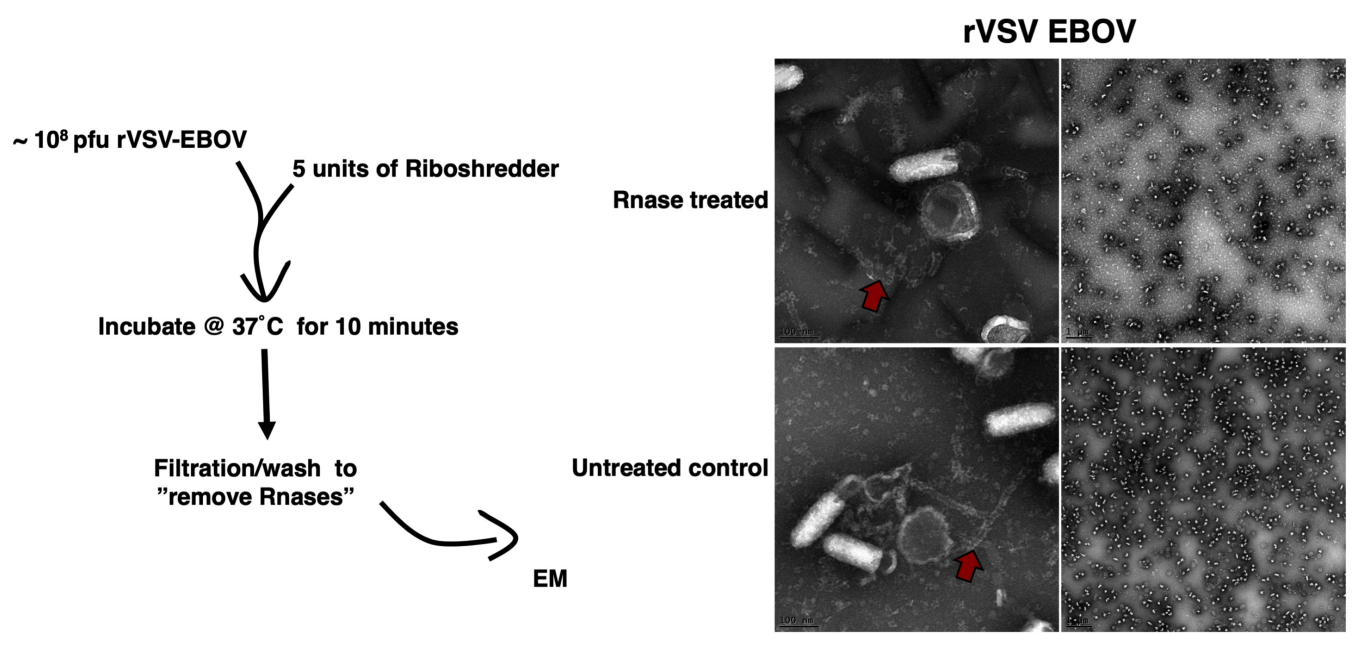

Figure 3.7. RNase treatment of sample preps failed to eliminate ribonucleoprotein complex from the virus samples. A. An overview on the RNase treatment process, where $10^{8}$ pfu of particles where incubated with 5 units of a cocktail of RNases (Riboshredder). B. Micrographs comparing treated sample preps to untreated samples. Structures that appear to be the ribonucleoprotein complex (red arrows) were found in treated (top panel) and non-treated (lower panel). 


\section{End-point PCR rules out cross-contamination between}

\section{rVSV/EBOV_GP and rVSV/MARV_GP preps.}

Target validation is a vital part of the target preparation that needs to be done before initiating SELEX. We sought to validate the presence of MARV and EBOV GP genes in their respective chimeric virus particles and rule out cross contamination between the two preps. Primers were designed to probe for the presence of marv gp and ebov gp genes in their respective chimeric particles through an end-point PCR assay (Fig 3.8A). A control primer set that probes the gene for the VSV L protein was included in all the PCR assays. The genomic RNAs from rVSV/EBOV_GP and rVSV/MARV_GP preps were extracted and used as templates for cDNA synthesis with random hexamers. The cDNAs were then used as templates for end-point PCR. Agarose gel electrophoresis of the PCR products showed correct amplicon sizes for marv gp-specific primers in rVSV/MARV_GP-derived cDNA (Fig 3.8B) and for ebov gp-specific primers generated amplicons with rVSV/EBOV_GP-derived cDNA (Fig 3.8C). Neither primer set amplified the corresponding fragment from the other genome. The $50 \mathrm{bp}$ amplicon generated by the marv $g p$ primer set $\mathrm{B}$ when used in combination with cDNAs from rVSV/EBOV_GP is not the right size for that primer set (174 bp), and thus represents an irrelevant, nonspecific amplification. cDNAs from both viruses generated amplicons for primers specific to the gene for the VSV L protein. These results suggest that the chimeric virus encodes their respective glycoproteins and rules out cross contamination between the two preps. These particles a therefore suitable for aptamer selection (Chapters $4 \& 5$ ), for chemical derivatization (next section), and for structural studies using electron microscopy (Paz et al, in progress). 


\begin{tabular}{|c|c|c|}
\hline Primer set & Target & Product length (bp) \\
\hline EBOV GP primer set A & EBOV GP & 268 \\
\hline EBOV GP primer set B & EBOV GP & 140 \\
\hline VSV L & VSV L & 321 \\
\hline MARV GP primer set A & MARV GP & 324 \\
\hline MARV GP primer set B & MARV GP & 174 \\
\hline
\end{tabular}
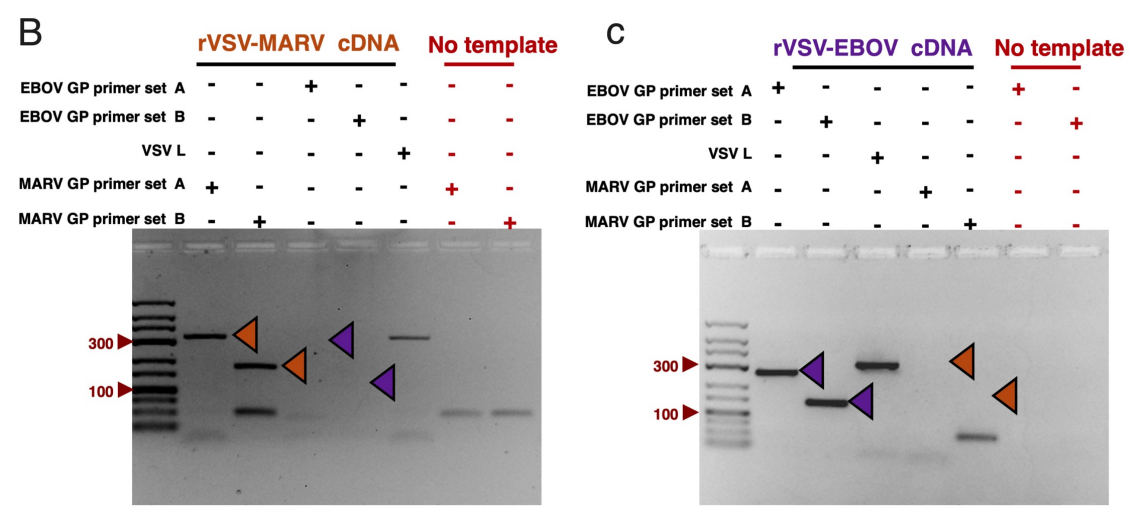

Figure 3.8. Agarose gel evaluation of endpoint PCR that utilized primers with specificity for the genes for marv $g p$ and ebov gp. A. Table outlines the primer sets and the expected amplicon sizes. There are two primer sets for ebov $g p$ colored in (purple), two for marv gp (orange), and a single set for vsv l gene. B Two marv gp specific primer sets (A and B) generated PCR products with cDNA templates from rVSV/MARV GP (amplicon indicated by orange triangle). Control set of primers specific for EBOV GP failed to generate PCR products (purple triangles). C. ebov gp specific primer sets generated products only with rVSV/MARV_GP derived cDNA (purple triangle). The $v s v l$-specific primers produced amplicons with cDNAs from both chimeric virus particles. 


\section{Chemical biotinylation of virus surfaces.}

Bead-immobilized virus particles could serve as excellent reagents for affinity capture of GP-binding aptamers and other ligands. Therefore, we developed two methods for immobilizing chimeric viral particles on paramagnetic beads. In the first method, rVSV/MARV_GP particles were chemically biotinylated and immobilized on streptavidin-coated paramagnetic Dyna beads. The chemical biotinylation approach requires the extent of the biotinylation reaction to be adequate for capture, but not too much to affect the virus infectivity. In the chemical biotinylation approach, primary amines on the virus surface proteins were labeled with biotin N-hydroxysulfosuccinamide ester (sulfo-NHS biotin) to generate a stable amide bond. Six concentrations of sulfoNHS biotin representing 2-fold serial dilutions were incubated with a fixed amount of virus particles $\left(10^{6} \mathrm{pfu}\right)$ and incubated overnight at $4^{\circ} \mathrm{C}$ in an alkaline buffer (Fig 3.9A). Excess biotin was removed through molecular weight cut-off filtration. Plaque assays were used to evaluate the impact of biotinylation on virus infectivity. The plaque assay shows a decrease in infectivity for the treatments with 31 and $63 \mu \mathrm{M}$ sulfo-NHS-biotin, but a significant decrease in virus infectivity in reactions with higher concentrations (Fig 3.9B), likely due to disruption of the virus surface proteins. 
A

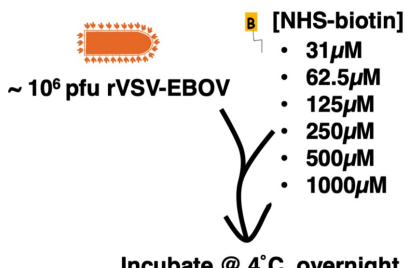

Incubate @ $4^{\circ} \mathrm{C}$ overnight PBS pH 7.5

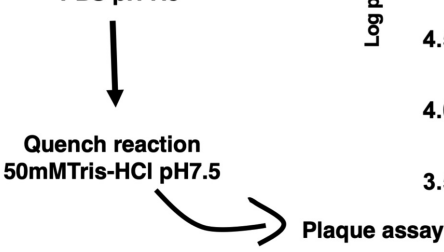

B

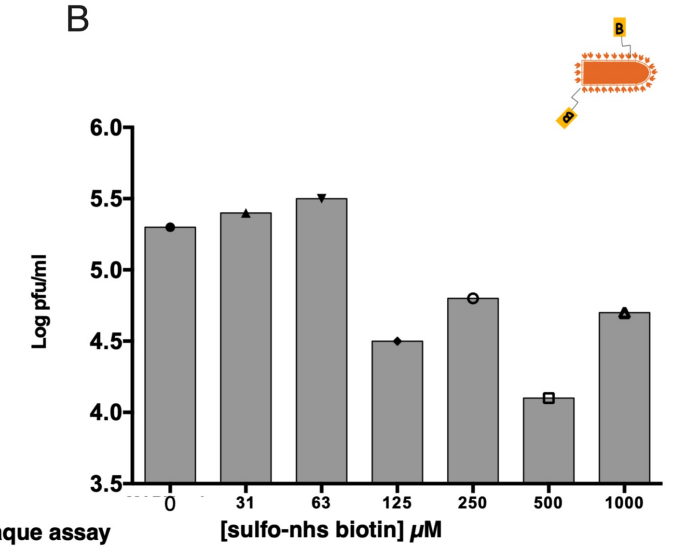

Figure 3.9. Biotinylating surface Lys on viral GP to enable capture by streptavidincoated beads. A. To optimize the chemical virus surface biotinylation process, different concentrations of sulfo-NHS-biotin were reacted with $10^{6}$ pfu of purified virus particles overnight at 4 degrees. B. The impact of surface modification on the virus infected was assessed through a plaque assay. Biotin concentrations at $125 \mu \mathrm{M}$ and above reduced virus infectivity. 
To determine whether the biotinylated virus particles display sufficient biotin for effective capture by streptavidin beads, increasing amounts of streptavidin-coated beads $(25,50$, and $500 \mathrm{mg})$ were incubated with $10^{5}$ pfu of biotinylated particles. The beads were removed with a magnetic bar after an hour incubation with the virus particles. To determine the capturing efficiency, plaque assays were performed on the left-over virus particles in the supernatant and compared with control non-biotinylated particles that were incubated with beads. The plaque assay shows a decrease in pfu in the biotinylated particles with increasing beads concentration. In contrast, increasing beads concentration did not have any noticeable impact on the pfu for non-biotinylated virus particles (Fig 3.10). Together, these results suggested that the virus particles displayed a significant amount of biotin for effective capture. 


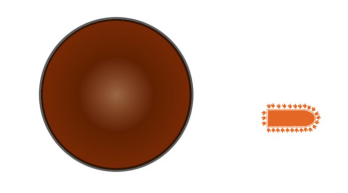

rVSV/MARV_GP [streptavidin coated dyna beads] $\mu \mathrm{g}$
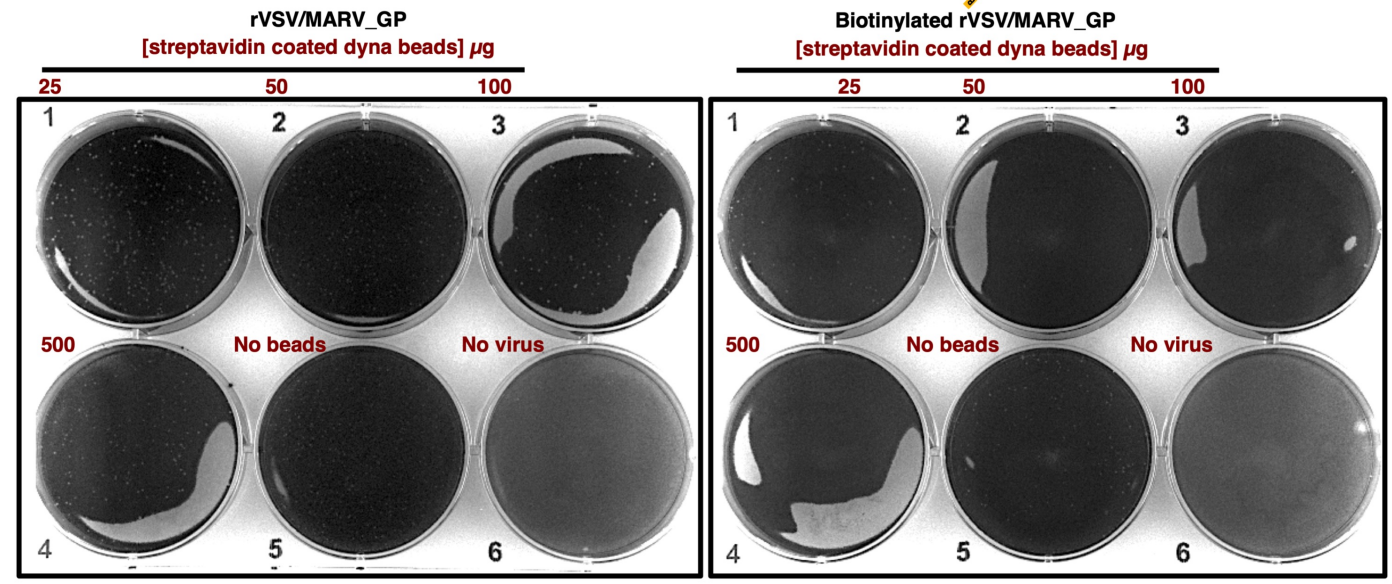

Figure 3.10. Plaque assays were used to evaluate whether virus surface-displayed biotin was sufficient for facilitating immobilization on the surface of SA-coated beads. Biotinylated virus particles (right) and non-biotinylated virus particles (left) were incubated with increasing amounts of SA-coated paramagnetic beads. Plaque assay was used to determine the number of virus particles left after beads were removed by a magnet. Increasing SA-coated beads concentrations decreases the number of plaques in biotinylated virus particles. In contrast, SAcoated beads did not affect the number of plaques in non-biotinylated virus particles. 
In the second method, rVSV/EBOV_GP virus particles were immobilized on protein Gcoupled Dyna beads coated with KZ52 (EBOV GP-specific monoclonal antibody). The immobilized particles were utilized as selection targets in beads-based SELEX. Details of the virus immobilization process and the selection process are presented in chapter 5 .

\section{Conclusion.}

The virus prep quality and the integrity of the virus particles are essential for whole-virus SELEX. The experimental work presented here shows that a three-step purification process is capable of removing contaminant proteins and cellular debris, resulting in a cleaner prep compared to a two-step purification process. However, preps from the threestep purification process also yielded broken particles and genome-like RNP structures. Longer centrifugation times, gentler buffer exchange approach, and RNase treatment failed to remove or separate the virus genome-like structures and the damaged particles from a full intact virus particle. Other factors not evaluated here, such as freeze-thaw, buffer composition, and the effects of the reagents used for the negative stain EM analysis, could also be contributing to the fragmentation of the virus particles. This work did not evaluate the impact of these factors on virus morphology, but ongoing structural studies on filovirus GP using electron microscopy will examine these factors (Paz, in progress). Finally, the work demonstrates that the surfaces of enveloped viruses can be modified with biotin through a reaction between primary amines on virus surfaces and NHS-biotin and that the biotinylated virus particles can be immobilized on SA-coated beads surfaces. 


\section{Materials and methods}

\section{Virus propagation/purification}

Virus was propagated in human embryonic kidney cell line (HEK293FT) (Invitrogen, Carlsbad, CA USA). Cells were maintained in standard growth media containing Dulbecco's Minimum Essential Medium (Sigma, St Louis, MO, USA) supplemented with 10\% fetal bovine serum (FBS) (Sigma), 2 mM L-glutamine, $1 \mathrm{mM}$ non-essential amino acids, and $1 \mathrm{mM}$ sodium pyruvate (all from Gibco, Life Technologies, Grand Island, NY, USA). Virus supernatant was propagated, purified, and concentrated as described elsewhere ${ }^{5}$. Briefly, $>90 \%$ confluent monolayer 293 FT cells were infected with the virus at a 0.01 multiplicity of infection (MOI) in a serum-free media for 1 hour. The supernatant was removed after incubation and replaced with cell growth media.

After we observed $\sim 50 \%$ cytopathic effects (cell rounding) on the cells, we collected the supernatants and centrifuged them at $3.5 \times 10^{3} \mathrm{rcf}$ for $10 \mathrm{~min}$ to pellet denser cellular debris. We filter the cleared supernatant through a $0.2 \mu \mathrm{m}$ membrane to further clear cellular debris. The partially purified crude virus was pelleted through $12 \%$ iodixanol cushion using a fixed angle rotor by spinning at $3 \times 10^{4} \mathrm{rcf}$ for $2 \mathrm{hr}$. This was followed by further purification by ultracentrifugation through $12 \%-35 \%$ continuous iodixanol gradient using a swinging bucket rotor at $1.6 \times 10^{5} \mathrm{x}$ g for $2 \mathrm{hr}$. A cloudy band observed $\sim$ mid-gradient, representing the virus was collected. To collect the cloudy band, the top layer is carefully pipetted without disturbing the cloudy band. Following that, a fresh pipette tip is used to carefully pipette the cloudy band. (For the fractionation experiment, the fractions were collected with a needle perforated on the side of the centrifugation tube 
right under the cloudy band. Samples were then allowed to drip out under gravity.) This iodixanol was removed by centrifugal filtration using the $100 \mathrm{kDa}$ Amicon ultracel PL membrane.

\section{Buffer exchange through centrifugal filtration}

Centrifugal filtration was executed using $100 \mathrm{kDa}$ molecular weight cut-off membrane as recommended by the manufacturer. Amicon ${ }^{\circledR}$ Ultra $100 \mathrm{kDa}$ centrifugation filters (Millipore Sigma) were pre-washed with PBS. The isolated virus band $(\sim 1 \mathrm{~mL})$ was diluted by adding $4 \mathrm{~mL}$ of PBS. The samples were then added to the pre-washed filters and spun for $30 \mathrm{~min}$ at $3000 \mathrm{rcf}$. An additional $2 \mathrm{~mL}$ of PBS was added to the samples in the filter column and spun for an additional $30 \mathrm{~min}$ (wash step). The samples were washed a total of three times by repeating this step. The resulting supernatant was collected by reversing the filter to a new vial and collected for further analysis.

\section{Plaque assay}

For plaque assays, Vero-E6 cells were plated in 6 well plates and allowed to grow to $\sim 90 \%$ confluency. The confluent cells were infected with various numbers of chimeric virus particles (10-fold serial dilution spanning $10^{7}$-fold range). For the infection, $500 \mu 1$ of viral dilutions in a serum-free media were incubated with confluent cells at regular cell growth conditions $\left(37^{\circ} \mathrm{C}\right.$ and $\left.5 \% \mathrm{CO}^{2}\right)$. After adsorption of the virus for 1 hour, the cells were overlaid with $1 \mathrm{ml}$ of $0.9 \%$ methylcellulose in standard cell growth media followed by incubation at $37^{\circ} \mathrm{C}$. Chimeric VSV plaques were fixed in $20 \%$ paraformaldehyde and visualized by staining monolayers with a $1 \%$ crystal violet solution on day 3 after infection. Plaques were counted and virus titer determined by the equation below.

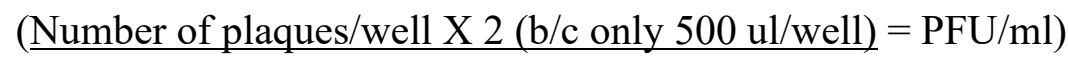




\section{Dilution}

\section{Negative Stain Electron Microscopy (performed by Mandy Paz)}

Purified chimeric VSV virus particles $(5 \mathrm{uL})$ were deposited on carbon-coated 200 mesh copper grids (Electron Microscopy Sciences, Hatfield, PA 19440), which had been freshly glow discharged for $45 \mathrm{sec}$ at 0.39 mBarr using a Pelco Easiglow Glow Discharge Cleaning System (Ted Pella) set to $15 \mu \mathrm{A}$. The virus samples were incubated on the grid for $2 \mathrm{~min}$, then wicked using filter paper (Millipore Sigma). Samples were immediately stained with 5 uL NanoW (Nanoprobes Yaphank, NY 11980-9710 USA) for another 2 min and wicked dry. The stained samples were visualized on a JEOL JEM-1400 Transmission Electron Microscope operated at $120 \mathrm{kV}$. Micrographs were acquired manually using different magnifications on a Gatan Ultrascan 1000 CCD camera (Gatan, Inc, Pleasanton, CA) .

\section{Acknowledgement}

A special thank you to Amanda Paz, who performed all the electron microscopy analysis.

\section{Addendum}

Future experiments for this work would continue with the efforts to identify and the source of damage particle and possible solutions. As described in above, factors such as the freeze thaw and the reagents used for negative could be damaging the particles. We will work with Dr. Tommy White's team to try other forms of staining if the current method is not ideal. Finally, we have discussed adding a size exclusion purification as a 
fourth step to help eliminate the ribonucleoprotein complex and damaged virus particles in the sample prep. 


\section{Reference}

(1) Wang, J.; Rudzinski, J. F.; Gong, Q.; Soh, H. T.; Atzberger, P. J. Influence of Target Concentration and Background Binding on In Vitro Selection of Affinity Reagents. PLoS One 2012, 7 (8). https://doi.org/10.1371/journal.pone.0043940.

(2) Garbutt, M.; Liebscher, R.; Wahl-Jensen, V.; Jones, S.; Möller, P.; Wagner, R.; Volchkov, V.; Klenk, H.-D.; Feldmann, H.; Ströher, U. Properties of ReplicationCompetent Vesicular Stomatitis Virus Vectors Expressing Glycoproteins of Filoviruses and Arenaviruses. J. Virol. 2004, 78 (10), 5458-5465. https://doi.org/10.1128/JVI.78.10.5458-5465.2004.

(3) Schnell, M. J.; Buonocore, L.; Kretzschmar, E.; Johnson, E.; Rose, J. K. Foreign Glycoproteins Expressed from Recombinant Vesicular Stomatitis Viruses Are Incorporated Efficiently into Virus Particles. Proc. Natl. Acad. Sci. U. S. A. 1996, 93 (21), 11359-11365. https://doi.org/10.1073/pnas.93.21.11359.

(4) Ilinykh, P. A.; Shen, X.; Flyak, A. I.; Kuzmina, N.; Ksiazek, T. G.; Crowe Jr., J. E.; Bukreyev, A. Chimeric Filoviruses for Identification and Characterization of Monoclonal Antibodies. J Virol 2016. https://doi.org/10.1128/JVI.00101-16.

(5) Diallo, J.-S.; Vähä-Koskela, M.; Le Boeuf, F.; Bell, J. Propagation, Purification, and In Vivo Testing of Oncolytic Vesicular Stomatitis Virus Strains. In Methods in molecular biology (Clifton, N.J.); 2012; Vol. 797, pp 127-140.

https://doi.org/10.1007/978-1-61779-340-0_10.

(6) Strobel, B.; Miller, F. D.; Rist, W.; Lamla, T. Comparative Analysis of Cesium Chloride- and Iodixanol-Based Purification of Recombinant Adeno-Associated Viral Vectors for Preclinical Applications. Hum. Gene Ther. Methods 2015, 26 (4), 
147-157. https://doi.org/10.1089/hgtb.2015.051.

(7) Janice E. Lawrence and Grieg F. Steward. Purification of Viruses by Centrifugation. MAVE Chapter 17, 2010, 166-181. 2010. https://doi.org/10.4319/mave.2010.978-0-9845591-0-7.166. 


\title{
Chapter 4: Differential recognition of MARV GP and EBOV GP by an ssDNA aptamer
}

This chapter will be modified for submission. Anticipated citation information: 'Differential recognition of MARV GP and EBOV GP by an ssDNA aptamer', by Kwaku

D. Tawiah, Amanda Paz, David Porciani, Carolina Camargo, Margeret J. Lange, Alexander Bukreyev and Donald H. Burke.

\begin{abstract}
Filoviruses such as Ebola virus (EBOV) and Marburg virus (MARV) cause fatal hemorrhagic fever in humans, non-human primates, bats, and other mammals. Like many infectious diseases, early and accurate detection is crucial for disease treatments and the management of outbreaks. Rapid, simple, and low-cost, point-of-care diagnostics can complement the existing RT-PCR and immunoassay MARV diagnostic approaches, especially in resource-limiting settings. Aptamers are small, structured nucleic acid affinity probes generated through in-vitro combinatorial chemistry technology. Their unique properties make them attractive affinity probes to develop affordable diagnostic devices. In this work, we developed ssDNA aptamers that bind to the glycoprotein (GP) from MARV in its native biological state when the GP is displayed on virus particles or HeLa cells. We employed a dual platform analysis to identify true positives and to eliminate potential false positives. The identified aptamers do not bind to the GP of a closely related EBOV. The aptamer can detect MARV GP in a simple ELONA virus detecting assay.
\end{abstract}




\section{Introduction}

Marburg virus (MARV) is a filamentous, single-stranded, negative-sense RNA virus that belongs to the family Filoviridae, along with the Ebola virus (EBOV) and other species. Both MARV and EBOV cause severe hemorrhagic fever with high case fatalities (40$90 \%$ ) in humans and non-human primates ${ }^{1,2}$. MARV transmission occurs through close contact with infected bodily fluids. Transmission can happen between humans or animals, and it can be zoonotic as well ${ }^{3,4}$. The first documented outbreak of Marburg Virus Disease (MVD) occurred in 1967 in Germany and Serbia ${ }^{5,6}$. Since then, there have been several sporadic outbreaks happening mainly in sub-Saharan Africa (detailed in Chapter 1). The largest outbreak occurred in Angola from 2004 to 2005, in which there were 252 reported cases and a case mortality rate of approximately $90 \%{ }^{7}$. There are currently no approved therapeutics for MVD. However, monoclonal antibodies such as MR191 isolated from circulating B cells in the blood of an MVD survivor were shown to protect non-human primates against MARV infection ${ }^{8,9}$. Patients are given symptomspecific treatments and supportive care such as rehydration, balancing electrolytes, and blood transfusion to manage MVD. The FDA recently approved an rVSV-vectored vaccine, which showed great promise against EBOV in 2017 ${ }^{10,11}$. While disease presentation for all filovirus infections is the same, the current promising drugs (REGNEB3 and mAb114) $)^{12-14}$ and the vaccine (VSV-ZEBOV GP) ${ }^{15-17}$ for EBOV do not work for MARV. Therefore, appropriate interventions would require ascertaining the filoviral species responsible for the disease. 
Early and accurate diagnoses are critical for the treatment and management of filoviral disease outbreaks ${ }^{18}$. Other infectious diseases such as malaria and typhoid fever share similar symptoms with MVD, which makes it more crucial to confirm MVD, especially in a place like sub-Saharan Africa, where these diseases are endemic ${ }^{19}$. Laboratory diagnoses of MVD utilize RT-PCR based assays and immunoassays (ELISA). RT-PCR and ELISA have been very useful diagnostic methods ${ }^{20,21}$. However, they require advanced instrumentation and a cold chain, which can limit their deployment to resourcepoor contexts. Moreover, developing antibodies can be challenging, and the batch-tobatch variability associated with their production can affect the reliability and accuracy of immunoassays $^{22,23}$. The recent outbreaks of Ebola virus disease in sub-Saharan Africa and current Coronavirus disease (COVID-19) pandemic highlight the need for rapid, lowcost, and simple point-of-care virus detection devices. Such devices can complement the current diagnostic assays by resolving some of the limitations mentioned above. The development of such viral-detection point-of-care devices would require affinity probes (molecular recognition elements) that address the deficiencies associated with current detection platforms.

Filoviruses, including MARV, display homotrimer chalice-shaped spikes of heavily glycosylated glycoprotein (GP) on their surface. The GP is the only virus protein expressed on the surface of MARV, making it a principal target for diagnostics ${ }^{24}$ and for vaccine development. Aptamers represent a promising class of affinity probes for virus diagnostics ${ }^{25,26}$. They are single-chained oligonucleotides that fold into unique 3Dstructures and bind to their targets with strong affinity and specificity. Aptamers are 
chemically synthesized and can be generated through an in vitro selection process known as SELEX (Systematic Evolution of Ligands by Exponential enrichment ${ }^{27,28}$. Chemical synthesis eliminates batch-to-batch variability, and the in vitro SELEX process makes aptamers well suited for high-throughput development. Also, aptamers are smaller in size than larger probes, such as antibodies, and could potentially access epitopes that are otherwise inaccessible ${ }^{22}$. Aptamers have been developed against a wide range of targets, including small molecules ${ }^{29}$, purified proteins ${ }^{30}$, mammalian cells ${ }^{31,32}$, bacteria $^{33}$, and viruses $^{34,35}$. The interactions between viruses and their hosts during infection has proven to be a major target for aptamers. Aptamers have been developed to target different components of a virus (e.g., polymerases, glycoproteins, viral proteins and virus genomic structures). Virus-targeting aptamers have been exploited as therapeutics and as probes for basic research and diagnostics. For example, a recent report described a 2'fluoropyrimidine-modified RNA aptamer that binds to the dimeric soluble glycoprotein (sGP) of EBOV as a potential probe for EVD diagnoses ${ }^{36}$. Unlike EBOV, transcription of marv $g p$ results in a single product displayed as trimer on MARV surfaces. Moreover, GP amino acid sequences differ by approximately $30 \%$ between EBOV and MARV. Therefore, EBOV sGP binding aptamers are less likely to bind to MARV GP due to the structural differences between the two proteins.

This work describes the generation of MARV surface binding aptamers as alternative affinity probes for MVD diagnosis. Conventional SELEX for developing aptamers that target viruses utilizes purified proteins as the selection targets ${ }^{36}$. Expression and purification of transmembrane proteins, including glycoproteins, can be challenging. 
Some purification approaches require modification such as truncation or deletion of domains, which can impact protein structure. Using a recombinant protein as a SELEX target is not a guarantee for binding to the native target because of possible conformational heterogeneity ${ }^{37}$. Adopting SELEX conditions and targets that mimic the application conditions of the intended aptamer increases the likelihood that the resulting aptamer would function as intended. Here, we selected ssDNA aptamers against MARV GP displayed on purified vesicular stomatitis virus (rVSV/MARV_GP) ${ }^{10,38}$. Subtractive steps against nitrocellulose filters and native VSV were performed to improve specificity. We employed a multi-assay screening platform to identify candidate aptamers while minimizing false positives, thereby improving the effectiveness of the post-selection screens for desired aptamers. The identified aptamers bind to MARV GP, but not a related GP from EBOV. Also, two of the identified aptamers do not compete with each other for access to the virus, thus indicating at least two non-overlapping epitopes. Finally, we show that a MARV GP targeting aptamer can be utilized as a molecular recognition element in a simple Enzyme-Linked Oligonucleotide Assay (ELONA) ${ }^{39}$. Our work represents the first demonstration of an aptamer-based MARV surface recognition probe.

\section{Results and discussion}

Selection of ssDNA aptamer against rVSV/MARV_GP viral particles.

Virus particles that displayed MARV GP were used as targets for the selections to preserve native GP structure during the selection. To minimize cellular debris and nonviral proteins in these preps, virus particles were purified by density gradient 
ultracentrifugation, and their integrity was assessed using electron microscopy (Fig 4.1). Electron micrographs showed high numbers of particles present per grid. Most of the particles maintain the classic bullet-shaped morphology of rhabdoviruses

(Fig 4.1, Fig S4.1). We performed 11 rounds of positive selections for aptamers with an affinity for purified rVSV/MARV_GP. The selection utilized a ssDNA library with a $40 \mathrm{~N}$ random region flanked by $24-\mathrm{nt}$ primer binding sites. Subtractive steps against VSV particles and nitrocellulose filters were performed to deplete non-MARV GP targeting sequences, thereby increasing specificity. The 3' primer binding site (3'pbs) of the library was annealed to a complementary oligodeoxynucleotide (ODN) (1.3-fold excess) during the thermal denaturation step to minimize the impact of the 3' pbs on the aptamer structure and to entrain the library to yield aptamers suitable for applications that require similar annealing, such as immobilization onto beads or nanoparticles for viral capture and detection. 


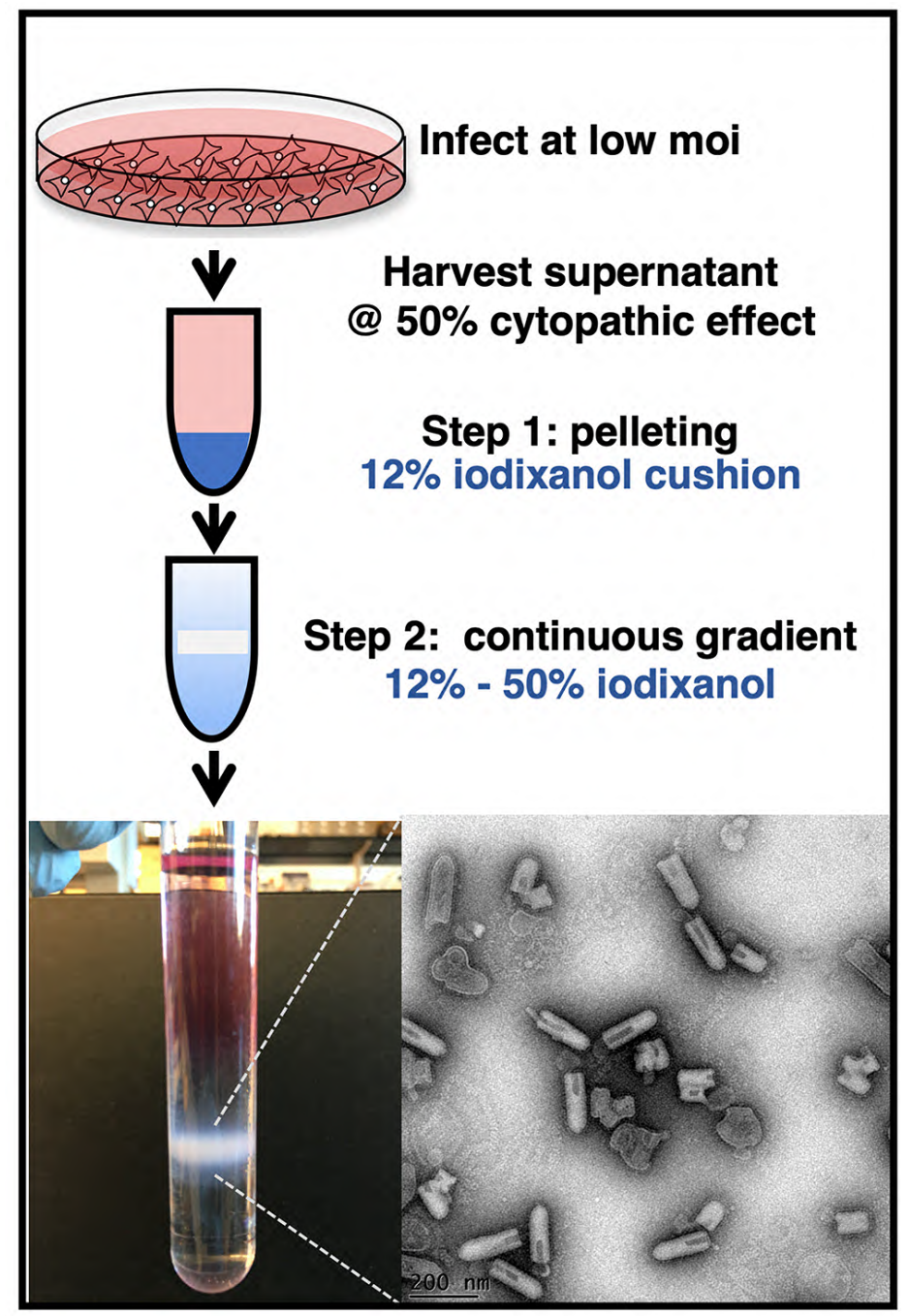

Figure 4.1. Overview of the virus propagation, purification and evaluation process. Virus particles were propagated in 293FT cells and subjected to a three-step purification process. Purification utilizes iodixanol density gradient. Virus Prep quality was assessed through electron microscopy. 
In each round, the thermally renatured ssDNA library was incubated with virus particles and passed through $\mathrm{KOH}$ treated nitrocellulose filters to isolate target-bound sequences. We observed that $\mathrm{KOH}$ treatment reduced the non-specific binding of ssDNA to the filters, consistent with previous reports ${ }^{41}$. The bound sequences were recovered and amplified for use in the subsequent rounds. Nitrocellulose subtractive step was performed before every positive round to deplete sequences with affinity for the nitrocellulose filters. Additional subtractive steps were performed during rounds 5, 7, 9, and 11 to deplete sequences with affinity for VSV. The number of virus particles used in each round was decreased throughout the selection to systematically increase the selection stringency. Details of the selection conditions are outlined in Table S4.1. A summary of the selection steps and the overall selection scheme is shown in Fig 4.2A and 4.2B. 


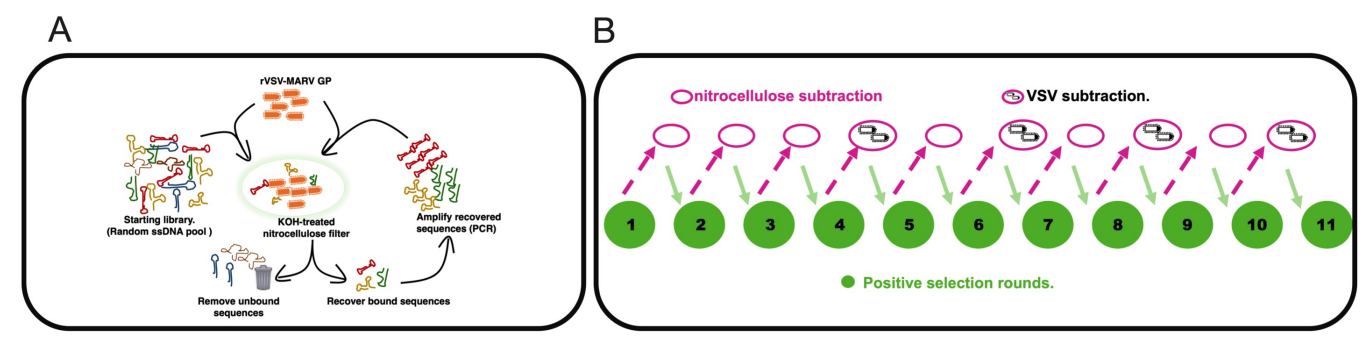

Figure 4.2. Overview of the selection strategy. A. In each selection cycle, the evolving ssDNA library (initially $\sim 10^{15}$ species) was incubated with rVSV/MARV viral particles; bound complexes were captured on $\mathrm{KOH}$-treated nitrocellulose. B. 11 rounds of positive selection and 10 subtractive steps were performed. Additional subtractive steps against VSV were included after positive Round 4 and repeated every other round. 
Following the 11th round, target binding and specificity were evaluated for the round 11 population through a radiolabeled filter retention assay. Round 11 population and some intermediate population (Rounds 5, 7 and 9) failed to show signal binding over the starting library when binding assessment were done using low viral particle number $\left(10^{6}\right.$ pfu) (data not shown). However, the round 11 shows a strong dose-dependent binding to rVSV/MARV_GP viral particles relative to the starting library when assessed with high number of virus particles ( $10^{8} \mathrm{pfu}$ ) (Fig 4.3 top panel). In contrast, both populations showed no difference in virus dose-dependent signal when assessed against non-targeted viral particles (rVSV/EBOV_GP and VSV) and protein (bovine serum albumin) (Fig 4.3 lower panels \& Fig S4.2). The round 11 population binds to MARV GP and does not bind to related glycoproteins such as EBOV GP nor VSV GP. Therefore, the round 11 population was cloned for sequencing. Among 46 sequences obtained, one sequence was sampled twice, and the remaining were all unique sequences (Table S4.2). The binding of the individual sequences to the target virus particles were evaluated by filter retention assays, with the binding signal from the starting library serving as a baseline for comparison. Three sequences (MH9, MH10, and MH34) showed strong binding signals ( $>2$-fold over starting library), relative to the signal from the starting library. Binding signals for ten additional sequences was 2-fold or less over starting library) (Fig 4.4A). 


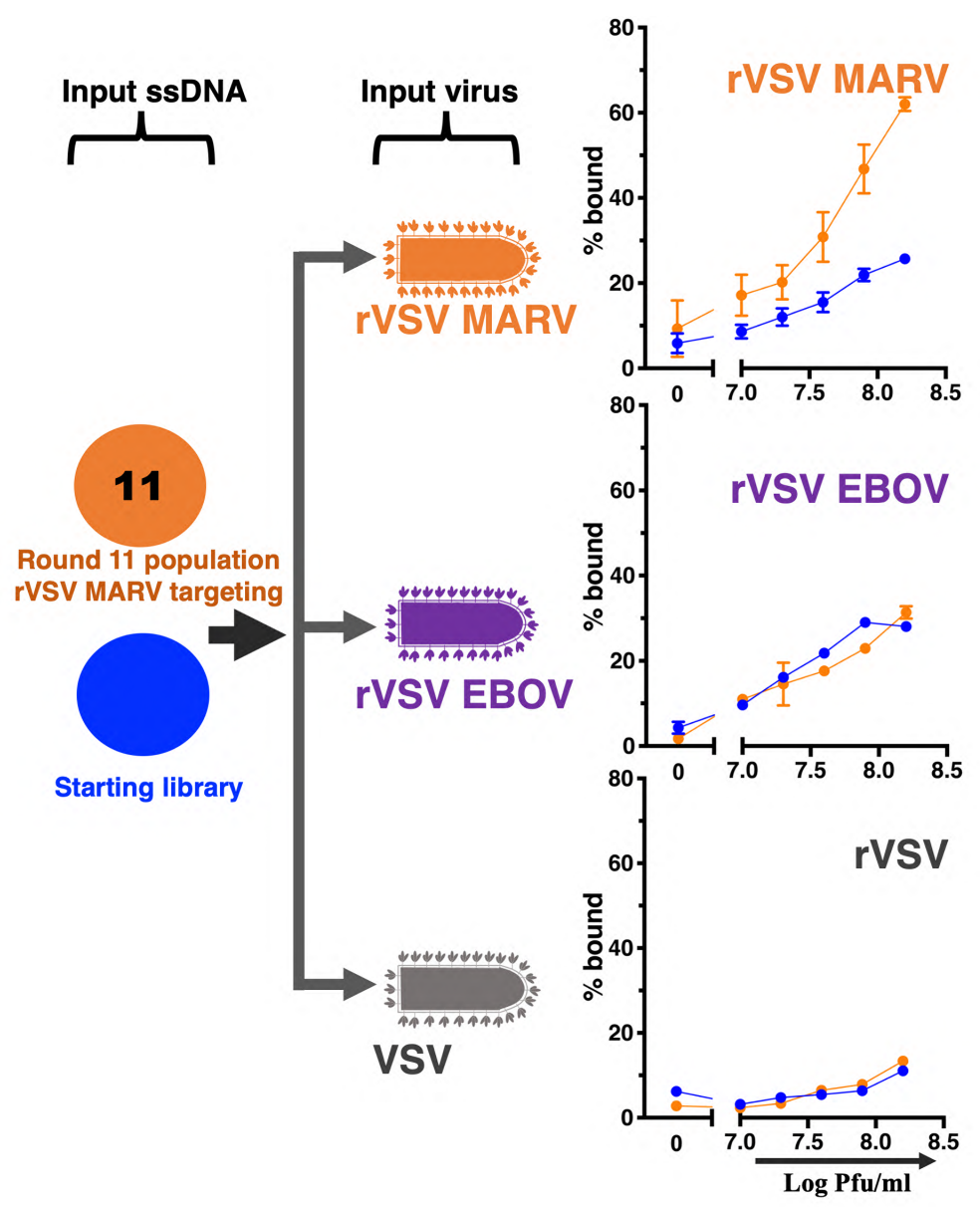

Figure 4.3. Binding specificity of ssDNA aptamer library selected for binding to MARV GP.Two radiolabeled ssDNA input libraries (round11, orange, and starting population, blue) were evaluated for dose-dependent binding to purified target (rVSV/MARV) and non-target (rVSV/EBOV and VSV) viral particles. Top panel, Round 11 population shows strong dose-dependent signal relative to starting library for binding to rVSV/MARV_GP. In contrast, both populations show the same low-level, dose-dependent binding signal when assessed for binding to rVSV/EBOV (middle panel) and VSV (bottom). Values are the mean \pm for three independent experiments for 
evaluations done on rVSV/MARV_GP and rVSV/EBOV_GP and one experiment on VSV.

Cross-platform screen identifies true MARV GP binders

When sequences are screened on the same platform on which they were generated, there is an inherent risk of obtaining false positives, such as binding to a non-target contaminant in the virus prep. To mitigate this risk, we developed an alternative screening platform that evaluated aptamer binding to HeLa cells that displayed filovirus GPs as targets. While some virus glycoproteins can be toxic to mammalian cells when overexpressed, HeLa cells have been reported to resist the cytopathic effects of filovirus GP expression ${ }^{42}$. As a first step towards developing this platform, GP expression levels 
that are tolerable to HeLa cells were established by transient transfection of GP plasmid at several concentrations $\left(0.1-5.0 \mu \mathrm{g}\right.$ per $10^{6}$ cells $)$, followed by evaluating GP expression by immunofluorescence flow cytometry at 24 and $48 \mathrm{hr}$ post-transfection. By this approach, transfection with $0.5 \mu \mathrm{g}$ of the plasmid gave the optimal GP expression with minimal cytopathic effects observed using a light microscope (Fig S4.3).

Next, flow cytometry was used to evaluate aptamer binding to MARV GP displayed on HeLa cells. Three sequences that showed strong binding in the filter retention assay promising (MH9, MH10, and MH34) and three additional sequences that were also somewhat promising (H13, H14, and H36) were annealed to a fluorescently labeled ODN and incubated with cells. Aptamer 'MH1' served as a non-binding control based on the filter retention screen above. Aptamers MH34 and MH36 retained binding to MARV GP when displayed on HeLa cells ( $\sim 3$-fold over non-binding control aptamer) (Fig 4.4C). In contrast, MH9 and MH10, which showed strong binding in the filter retention assay $(\sim 3-$ fold over starting library), bound only modestly to MARV GP when displayed on HeLa cells ( $<1.5$-fold over non-binding control) (Fig 4.4B and 4.4C).

This cross-platform screen identified a class of ssDNA aptamers that bind to MARV GP. Aptamer MH34 was chosen for further analysis, as it consistently showed a high binding signal in both the filter retention assay with virus particles and flow cytometry with MARV GP-expressing HeLa cells. This cross-platform screen both identified true positives and helped eliminate several potential false positives. Selective target recognition on MARV GP-expressing HeLa cells represent a more complex environment 
than purified proteins; therefore, these results are promising indicators that these aptamers may be able to detect their targets in a complex environment such as blood or serum. 

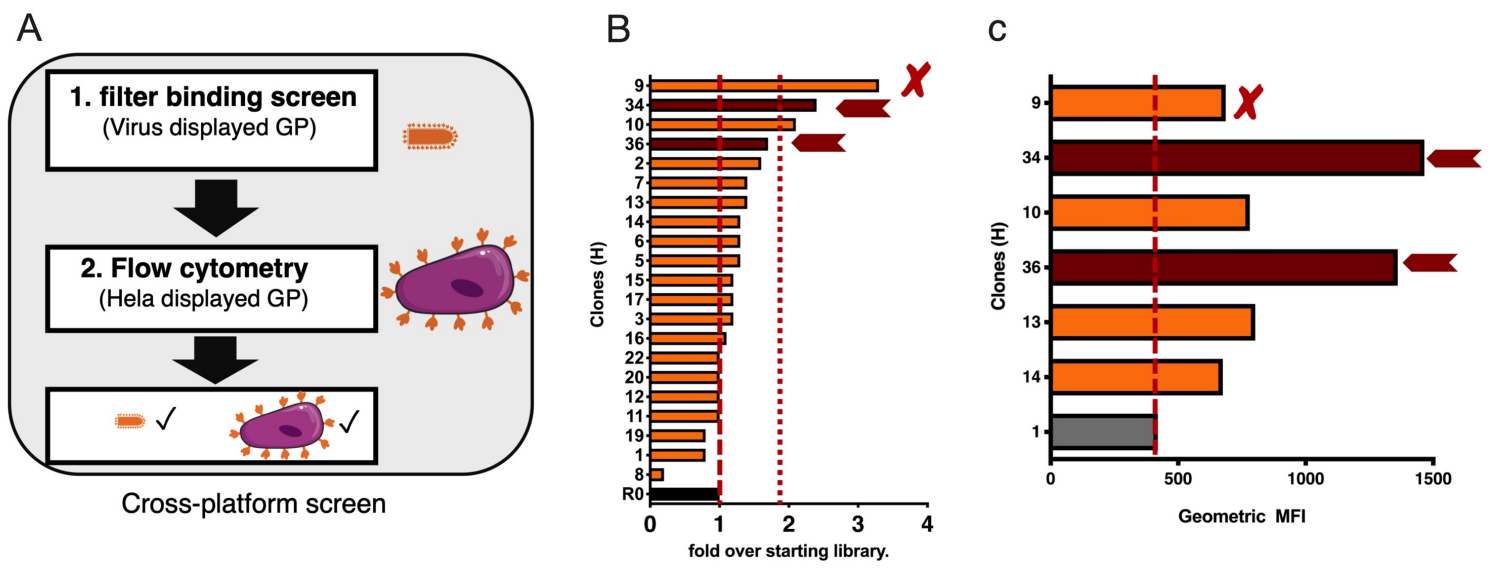

Figure 4.4. Post-SELEX, cross-platform screening approach A. identifies 'true positive' (arrows) aptamers that bind to MARV GP. B. Filter retention assay in which aptamers were screened for binding to chimeric VSV/MARV GP particles. Dotted line at 2x MFI. C. Flow cytometry assay in which fluorescently tagged aptamers were screened for their ability to label HeLa cells that express MARV GP. Arrows, 'true positives.' 'X,' false positive. 
Binding and specificity of full-length and truncated aptamer variants.

For potential use of MARV GP targeting aptamers in POC devices for MVD diagnoses, it is desirable that the aptamers can differentially recognize MARV GP from other GPs. Binding and specificity of MH34 to MARV GP relative to a non-binding scrambled control scMH34 were evaluated using multiple assays. First, radiolabeled MH34 was shown to bind to rVSV/MARV_GP particles with filter retention signal $\geq 2$-fold higher than scMH34 in a filter retention assay (Fig 4.5A). In contrast, there was no difference in the filter retention signal from MH34 or scMH34 against rVSV/EBOV_GP. Second, for the flow cytometry assay, MARV GP expressing HeLa cells were treated with MH34 or the scrambled variant. The cells treated with MH34 showed approximately 4-fold higher fluorescent intensities, relative to those treated with scMH34 (Fig 4B). In the flow cytometry assay, MH34 showed a slightly higher fluorescent intensity ( 2-fold) over the scramble variant when incubated with EBOV GP expressing-HeLa cells. We suspect the difference observed is due to the low-level binding of MH34 to HeLa cells since a similar binding phenotype was observed in cells that were transfected with an empty vector.

(Fig 4.5B). Third, MH34 binding to MARV GP was further confirmed through microscale thermophoresis (MST). Finally, the interaction between MH34 and MARV GP was validated through electrophoretic mobility gel shift assay (EMSA). Both assays utilized rVSV/MARV_GP particles as targets. (Fig S4.4). Next, the specificity of MH34 was evaluated by assessing binding to EBOV GP displayed on viruses (rVSV/EBO_GP) and on HeLa cells. Together, these results suggest that MH34 preferentially binds to MARV GP over EBOV GP. 
The aptamers in this study were selected in the presence of an anti-tail ODN annealed to their 3'pbs. To determine whether the functional structure of MH34 is independent of the 3'pbs, a 3'pbs-truncated variant (MH34 $\Delta 3$ 'pbs) was fluorescently tagged and evaluated for binding to MARV GP displayed on HeLa cells through flow cytometry. Fluorescence signal for this construct was $\sim 2$-fold higher than a non-binding control, similar to the parent structure. (Fig S4.4). Next, we truncated the 5'pbs of MH34 and MH36, annealed them to a fluorescently tagged ODN, and evaluated the binding of the truncated variants to MARV GP displayed on HeLa cells using flow cytometry. MH34 $\triangle 5$ 'pbs retained binding to MARV GP in this context ( 4-fold greater fluorescence signal than for scMH34 $\Delta 5$ 'pbs). In contrast, MH36 $\Delta 5$ 'pbs failed to bind to the MARV GP expressing HeLa cells (Fig 4.5C). These data establish that the functional core of MH34 is independent of both 5'pbs and 3'pbs, while MH36 requires at least some portion of the 5 'pbs to form a functional structure. 

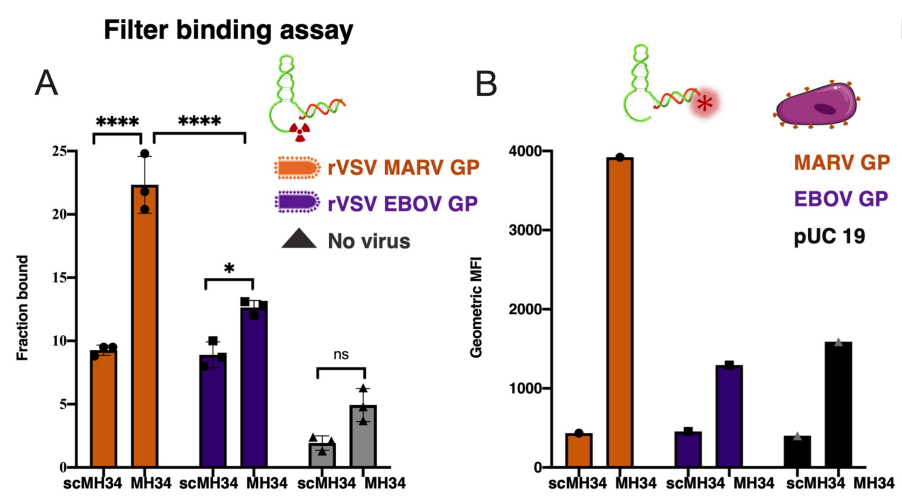

Flow cytometry

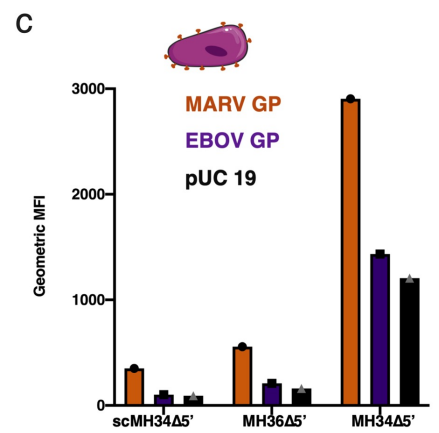

Figure 4.5. MH34 binding and binding specificity were assessed using multiple binding assays. Binding was validated on MARV GP (orange) and binding specificity were assessed on EBOV GP (purple) and filter/cells only (gray). A. filter retention assay was used to assess binding of MH34 and scMH34 against rVSV/MARV_GP (target), rVSV/EBOV_GP (non-target), and filter (background). B. flow cytometry was used to assess binding of MH34 and scMH34 to MARV GP-expressing HeLa cells (target), EBOV GP-expressing HeLa cells (non-target), and pUC19 transfected-HeLa cells (background). C. flow cytometry was used to assess binding of $5^{\prime}$ truncated sequences ( $\mathrm{MH} 34 \Delta 5$, MH36 $\Delta 5^{\prime}$ and scMH34 $\Delta 5^{\prime}$ ). For flow cytometry assays, representative geometric mean values are shown, out of a minimum of two independent experiments. For the filter retention assay, the values are the mean + for three independent experiments. Data comparison and analysis was done by two-way ANOVA with post hoc Tukey's test. Brackets with asterisks represent statistical differences: ns not significant; ${ }^{*} p<0.05 ; * * p<0.01 ; * * * p<0.001 ; * * * * p<0.0001$. 
MH34 and MH36 do not compete with each other for binding to virus surface.

MH 34 and 36 share no significant sequence identity (Table S4.2) or apparent secondary structures and are assumed to fold into distinct 3D structures. Competition experiments were performed to determine whether these two aptamers bind to the same epitope or different epitopes on the MARV GP surface.rVSV/MARV_GP viral particles were incubated with 3- to 100-fold excess unlabeled competitor prior to adding radiolabeled MH34. Radiolabeled MH34 failed to bind to rVSV/MARV_GP in the presence of 3-fold excess non-radiolabeled MH34, as expected for self-competition, but binding was unaffected by 100-fold excess scMH34. These data establish the utility of the assay. When excess MH36 was used as the competitor, binding of labeled MH34 was again unaffected (Fig 4.6), thereby establishing that MH34 and MH36 do not compete with each other for binding to MARV GP, likely indicating that they bind to distinct, nonoverlapping epitopes. 


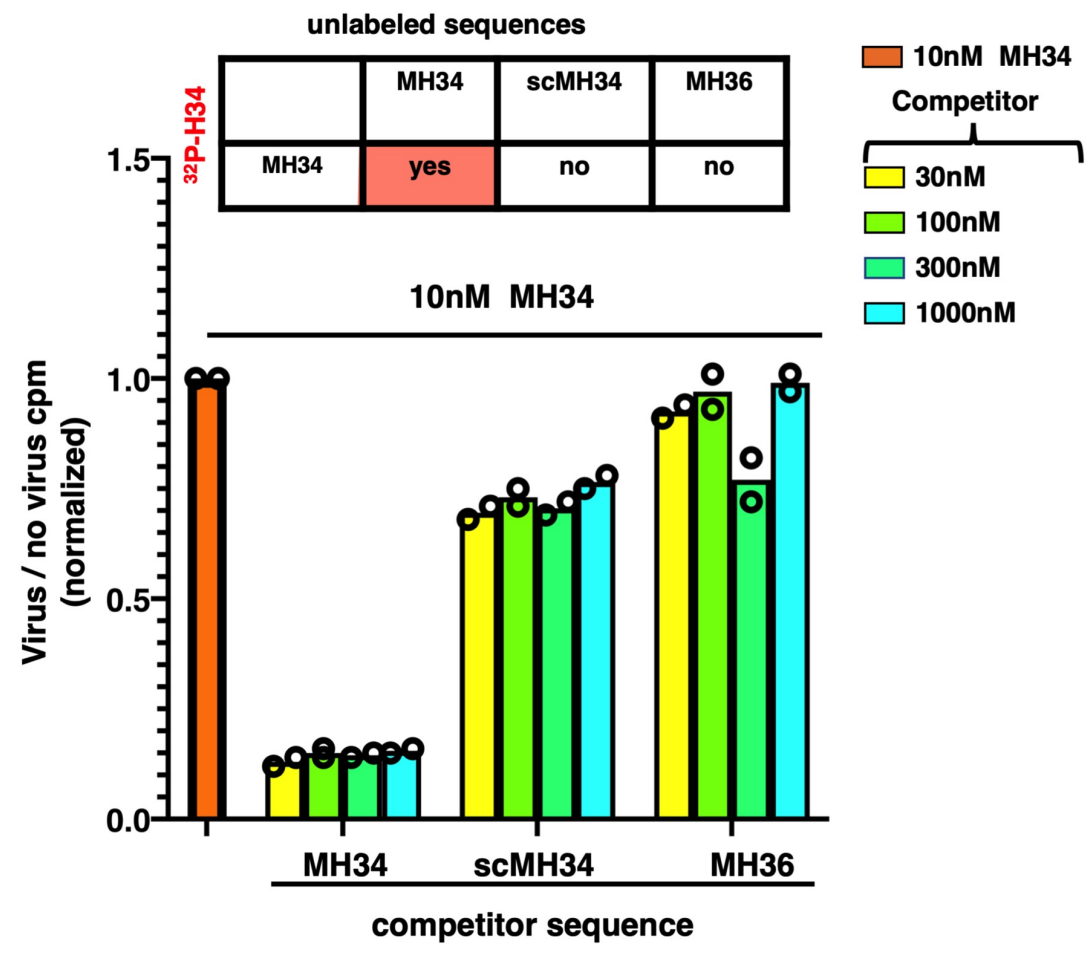

Figure 4.6. Competition assay shows that aptamers MH34 and MH36 bind to different epitopes of MARV GP. Plot shows the fraction of radiolabeled MH34 that binds the rVSV/MARV_GP viral particles in presence of the indicated, non-radiolabeled competitor at increasing concentration. Control scTAMP34, a non-binding scrambled version of MH34. The data represent 2 independent experiments $(n=2)$. 
MH34 reliably detects MARV GP displayed on viruses when used in an ELONA.

As an initial step towards determining whether aptamers that specifically interact with MARV GP could be used to develop low-cost sensors, we asked whether MH34 could be used as a molecular recognition probe in a simple Enzyme-Linked Oligonucleotide $\underline{\text { Assay }}$ (ELONA). Various concentrations of rVSV/MARV_GP viral particles (2-fold serial dilutions spanning a 1000-fold range) were captured onto MARV GP antibody-coated plate. The captured particles were probed with biotinylated MH34 or scMH34 annealed to an ODN complementary to the 5' pbs. Aptamer binding was detected through a streptavidin-tagged horseradish peroxidase activity. Conversion of 3,3',5,5'Tetramethylbenzidine (TBM) substrate into yellow product was visible to the naked eye and was quantified by measuring absorbance at $450 \mathrm{~nm}$ in every well as an indication of the concentration of aptamer present in that well. MH34 showed MARV GP specific signal over background signal from scMH34 in the presence of 2.0 to $7.5 \times 10^{6} \mathrm{pfu}$ of rVSV/MARV_GP, with a signal change that can be detected with the naked eye.

(Fig 4.7). MH34 showed a higher signal over the scMH34 when the virus particles were immobilized with a MARV GP neutralizing serum from guinea pigs (Fig S4.5). 

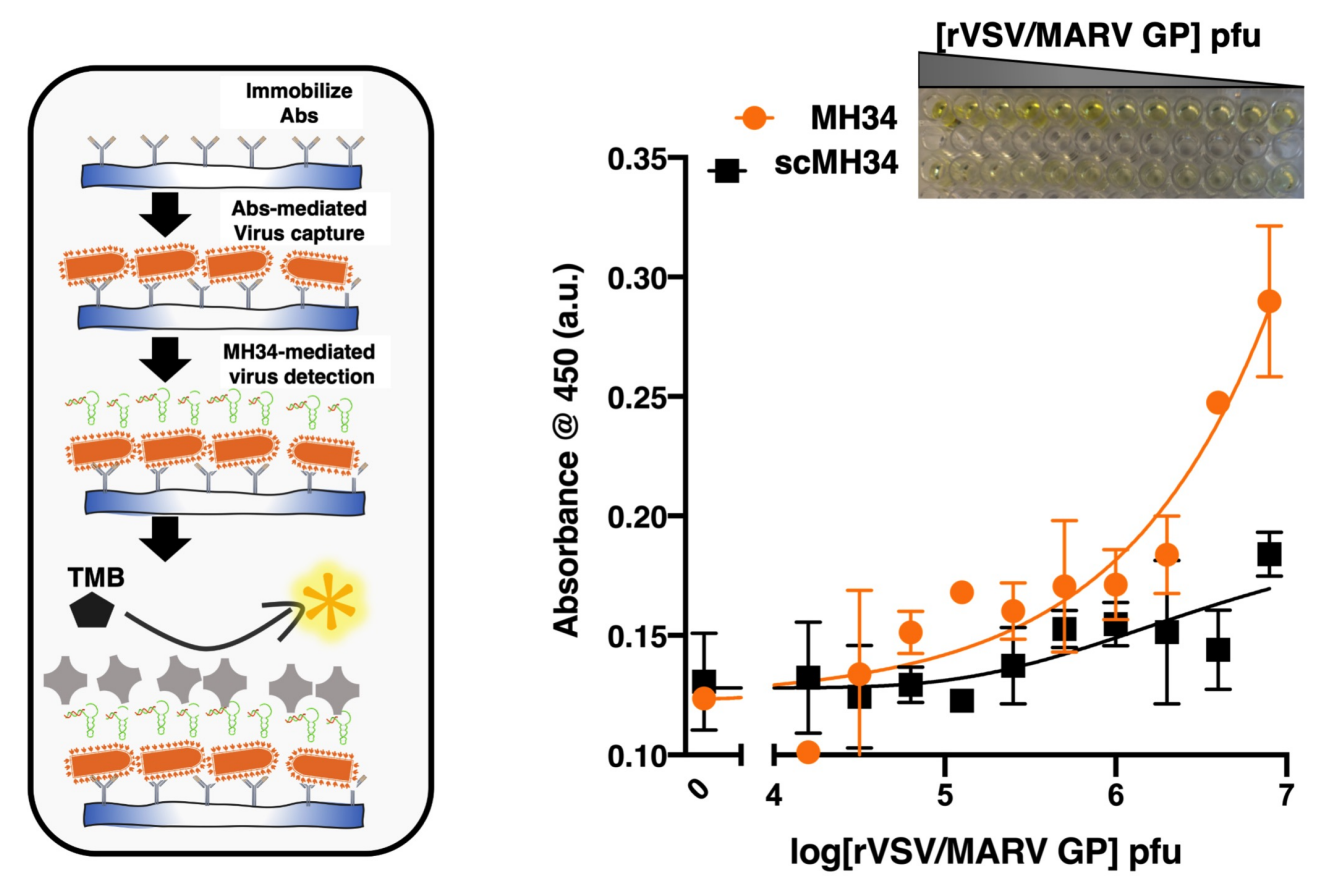

Figure 4.7. Scheme and experimental detection of rVSV MARV GP using antibodyaptamer based ELONA. A. scheme of the antibody-aptamer based MARV GP detecting ELONA platform. B. ELONA plots of MH34 and scMH34 used as molecular recognition elements. Virus particles were captured with MARV GP specific monoclonal antibody. The data represents three independent iterations of the experiment ( $\mathrm{n}=3$ ). (Fig S4.5) Virus particles were captured with anti-MARV GP serum. 


\section{Conclusion.}

Alternative affinity probes are needed that target virus surface proteins for developing low-cost POC devices and assays that can complement the existing PCR-based assays and immunoassays. Filovirus disease outbreaks have increased in recent years ${ }^{18}$. In addition, the current COVID19 pandemic and emerging viral diseases such as Nipah virus infection, Lassa fever, Zika fever, Hendra virus infection, and Rift Valley fever highlight the need for diagnostic assays that can be rapidly developed and easily deployed to remote and resource-limiting areas during outbreaks and pandemics.

This study describes a new class of aptamer-based affinity probes, that bind to MARV surface proteins. The aptamers were developed through 11 rounds of positive SELEX against rVSV/MARV_GP viral particles that also included depletion of species with affinity for VSV or nitrocellulose, A rigorous cross-platform post SELEX screening approach, which included nitrocellulose filter retention by viral particles, flow cytometry with GP-expressing cells, MST, EMSA, and ELONA identified and helped to prioritize true binders for further development.

The aptamers identified in this work (MHs), bind to GP from MARV and not to a closely related EBOV GP. The ability of MHs to differentiate between GPs from MARV and EBOV is particularly essential for developing MVD specific POC diagnostic devices. Binding analysis of two MHs and their minimized variants showed that Marv surface targeting aptamers retain selective binding to MARV GP in a relatively complex environment such as on cell surfaces. GP recognition by MH34 is independent of the $5^{\prime}$ 
and 3 'pbs sequences of the parent library, suggesting that it could potentially be truncated to 40 nt or less, while MH36 required at least a portion of the $5^{\prime}$ pbs. Finally, MH34 reliably detected rVSV/MARV_GP when used as a molecular recognition probe in an ELONA assay. These results establish a virus surface targeting-aptamer-discovery platform that could be extended to other emerging enveloped viruses.

\section{Addendum}

Aptamers that bind to MARV GP may block MARV infection. The current work only evaluates binding of the aptamers to MARV GP and potential utility as a detection probe. Our collaborators, Dr. Bukreyev's team at the University of Texas Medical Branch, Galveston have access to biosafety level 4 laboratories and have tremendous experience on screening for neutralizing antibodies for filoviruses. They will be evaluating MARV GP targeting aptamers for their ability to neutralize MARV infection. These evaluations would include other binding sequences from the evolved population. This work will be a follow-up would work or a potential addition to this work depending on the timeline for performing these experiments. 


\section{Supplementary information}

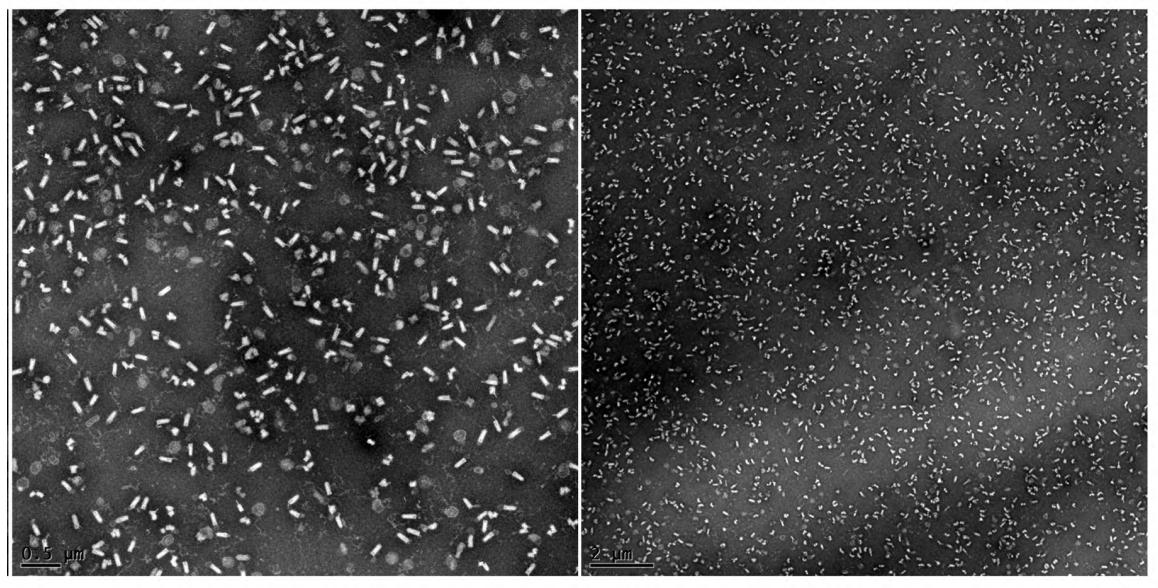

Figure S4.1. Electron micrographs of $\mathrm{rVSV} / \mathrm{MARV}$ GP in PBS following the virus purification process. 


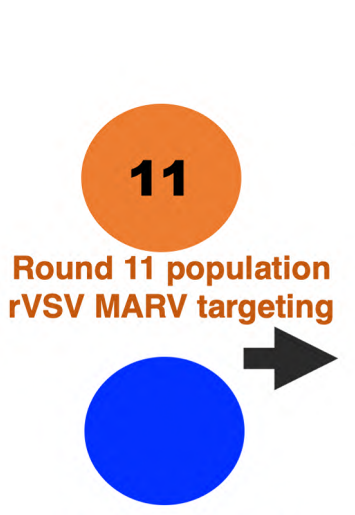

Starting library

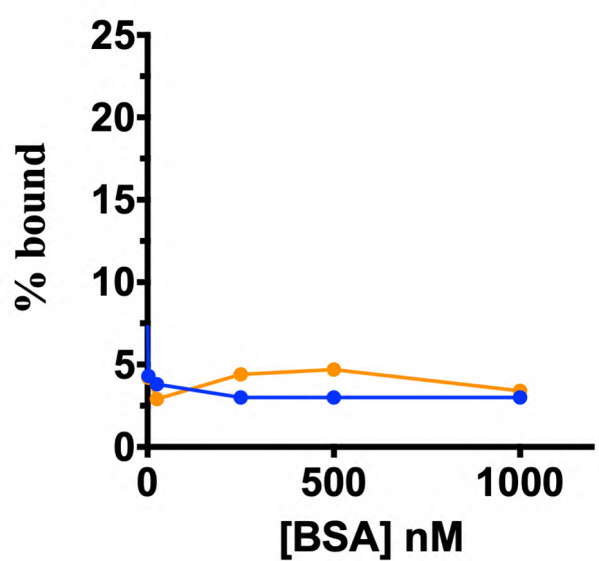

[BSA] nM

Figure S4.2. Analysis of the dose-dependent target and non-target binding of radiolabeled ssDNA from round 11 (orange) and starting population (blue). Round 11 population show (top panel) rVSV/MARV GP-dependent signal relative to starting library. Both populations show the same dose-dependent binding signal when assessed against (middle panel) rVSV/EBOV, (bottom) VSV, and BSA (Figure S2). 
a

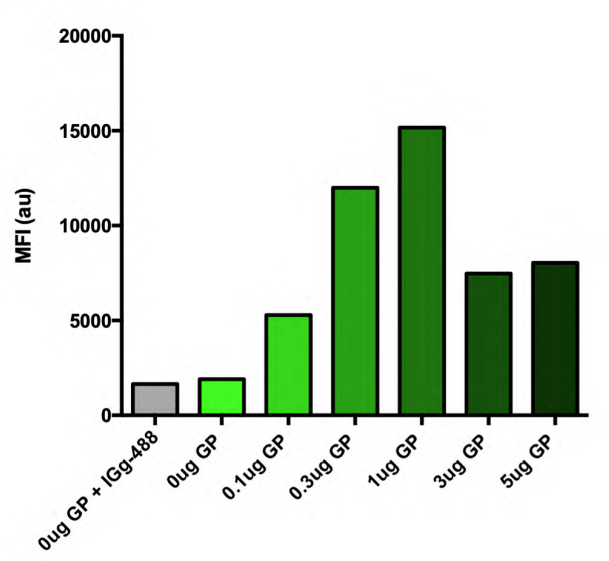

b

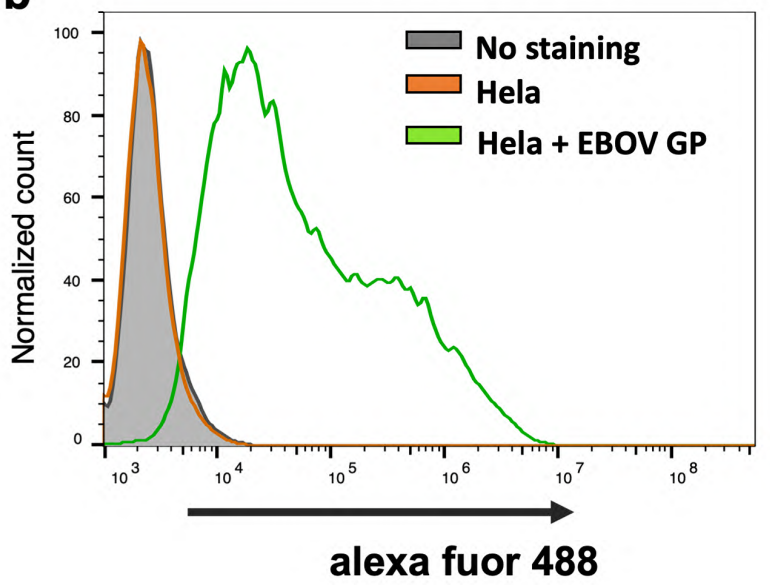

Figure S4.3. Transient transfection to establish filovirus GP-expressing HeLa cells and validation of staining through antibody-mediated cell staining. A. flow cytometry analysis of EBOV GP-expressing, 24 hours post transfection with varying concentrations EBOV GP. B. flow cytometry analysis HeLa cells, 24 hours post transfection with $0.5 \mu \mathrm{g}$ of EBOV GP. 

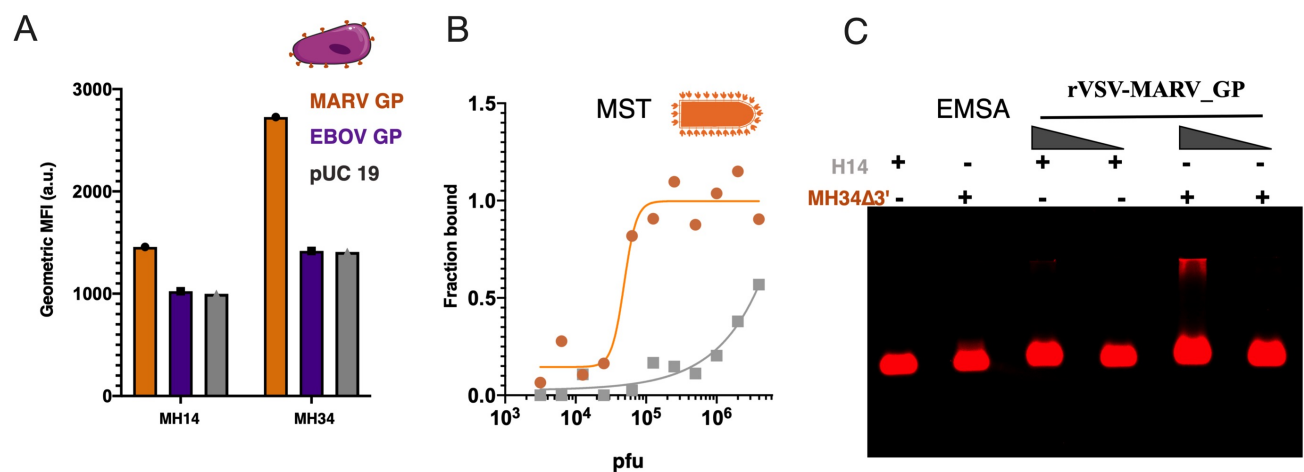

Figure S4.4. MH34 $\Delta 3^{\prime}$ binding and binding specificity were assessed using multiple binding assays. Binding was validated on MARV GP (orange) and binding specificity were assessed on EBOV GP (purple) and cells only (gray). A. Flow cytometry assay was used to assess binding of MH34 and MH14 (non-binding control). B. MST and C. EMSA assays were used to evaluate binding of MH34 33 to rVSV/MARV_GP particles. 


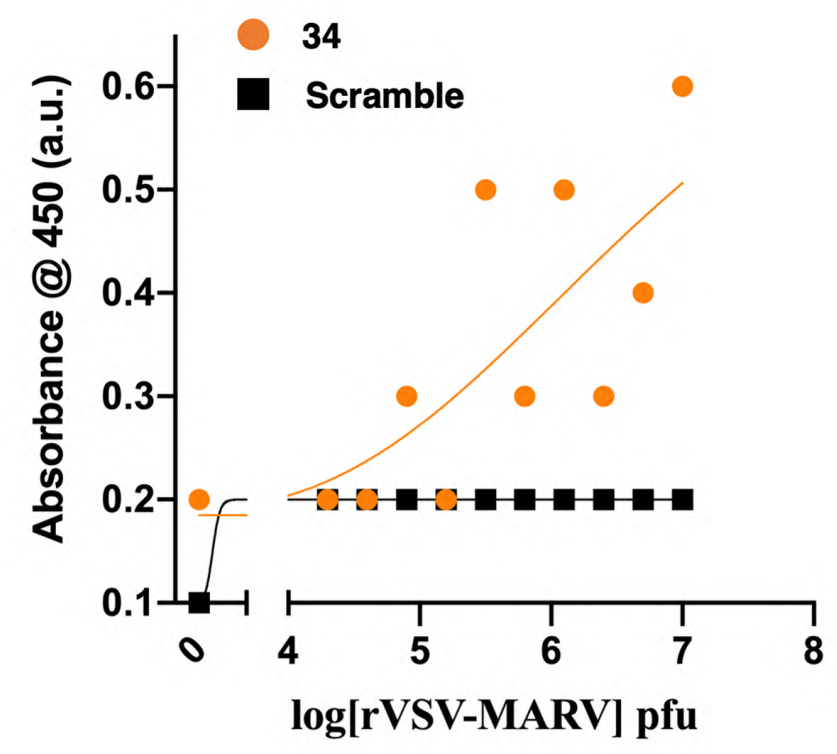

Figure S4.5. Scheme and experimental detection of rVSV MARV GP using antibodyMASTA based ELONA. A. scheme of the antibody-aptamer based MARV GP detecting ELONA platform. B. ELONA plots of MASTA34 and scMASTA34 used as molecular recognition elements. Virus particles were captured with MARV GP specific monoclonal antibody. (Figure S5) Virus particles were captured with antiMARV GP serum. 
Table S4.1. Selection conditions for MARV GP selection

$\begin{array}{lllll}\text { Round } & \text { Reaction volume }(\boldsymbol{\mu L}) & \text { [sSDA] } \boldsymbol{\mu M} & \text { [virus] pfu } & \text { Subtraction } \\ 1 & 100 & 10 & 6 \times 10^{6} & - \\ 2 & 100 & 5 & 2 \times 10^{6} & \text { nitrocellulose } \\ 3 & 100 & 3 & 5 \times 10^{5} & \text { nitrocellulose } \\ 4 & 100 & 3 & 5 \times 10^{5} & \text { rVSV } \\ 5 & 100 & 3 & 5 \times 10^{5} & \text { nitrocellulose } \\ 6 & 100 & 3 & 5 \times 10^{5} & \text { rVSV. } \\ 7 & 100 & 3 & 5 \times 10^{5} & \text { nitrocellulose } \\ 8 & 100 & 3 & 5 \times 10^{5} & \text { rVSV. } \\ 9 & 100 & 3 & 5 \times 10^{5} & \text { nitrocellulose } \\ 10 & 100 & 3 & 5 \times 10^{5} & \text { rVSV. } \\ 11 & 100 & 3 & 5 \times 10^{5} & \text { nitrocellulose }\end{array}$




\section{Table S4.2. Sanger sequencing results for MH clones. Sequences show represent the}

\section{random region of the library. See the material and methods section for the $5^{\prime}$ and 3}

\section{' primer binding sequences}

\begin{tabular}{|c|c|}
\hline Clone & $40 \mathrm{~N}$ part. $\left(5^{\prime}---->>3^{\prime}\right)$ \\
\hline$>\mathrm{M} 40 \mathrm{~N} 2{ }^{\prime} \mathrm{H} 1$ & CAGACAGTCAGGCAGGTACAAAATAGTACGACGGGACGCA \\
\hline$>\mathrm{M} 40 \mathrm{~N} 2{ }^{\prime} \mathrm{H} 2$ & GGGCAACCAGTGCTGAGGCTGTCGGTAGAGATCGTGATGT \\
\hline$>$ M4 ON2'H3 & TCAAAAGCACAGGTGAGGAGAAATAATTTGGGGGGTCTGG \\
\hline$>\mathrm{M} 4$ ON2'H5 & AAAGACAATGTGTGACAGCAAGCCGTACGATTGGGTGGTG \\
\hline$>$ M4 ON2'H6 & GGGGGTAGTACGGGAAGGGTTTCGTATACTGTTACTCAGA \\
\hline$>\mathrm{M} 4$ ON2'H7 & TGTAACAATATTGCAAGGCAGACGCCAAAAGGGAAATGGG \\
\hline$>\mathrm{M} 4$ ON2'H8 & CACAGGAGTGCATGTGCGCTTAGGAGGGACGTGTGGGTGA \\
\hline$>$ M4 ON2'H9 & AAAGGCGGATAAGAGTGGAATGGTCACCCAGGGTATGGGT \\
\hline$>\mathrm{M} 40 \mathrm{~N} 22^{\prime} \mathrm{H} 10$ & AGGGAATGGAGACAGACCTGTACACACATGGGTATACGTA \\
\hline$>\mathrm{M} 40 \mathrm{~N} 22^{\prime} \mathrm{H} 11$ & AAAGTAGGAAAAAGGCATGGAAGAGCCACAAGGCACTCAC \\
\hline$>\mathrm{M} 40 \mathrm{~N} 22^{\prime} \mathrm{H} 12$ & TACCAAAAGATGCAGGAGCTAGGATGGGCGCAAAGCGGTA \\
\hline$>\mathrm{M} 40 \mathrm{~N} 22^{\prime} \mathrm{H} 13$ & CCAAATGGGCGACGGGCATGGCTCCAAGATCGTCTGCGAC \\
\hline$>\mathrm{M} 40 \mathrm{~N} 22^{\prime} \mathrm{H} 14$ & TAGCCCAACTTGAGCAAGAGGTAAAGCGGAGTTTTGTATG \\
\hline$>\mathrm{M} 40 \mathrm{~N} 22^{\prime} \mathrm{H} 15$ & CCGAAGAGAGGACAGCAGATACACTTGGTTGAATAGCAAA \\
\hline$>\mathrm{M} 40 \mathrm{~N} 22^{\prime} \mathrm{H} 16$ & GGGTAGAGAGACGTGGGGCGTAGGATAAGCCGAGCGTCAT \\
\hline$>\mathrm{M} 40 \mathrm{~N} 2{ }^{\prime} \mathrm{H} 17$ & GTTGGGGAGCAAGTAAGCAGGACGTTCCGGCGACAAGGAA \\
\hline$>\mathrm{M} 40 \mathrm{~N} 22^{\prime} \mathrm{H} 18$ & GGCTGAGACAAAACCGGTGAGACGCGGTAACTCTGCTGTG \\
\hline$>\mathrm{M} 40 \mathrm{~N} 2{ }^{\prime} \mathrm{H} 20$ & TCACATGGAGTTGAGTCGGACTAGACAAGGATATGGCGCG \\
\hline$>\mathrm{M} 40 \mathrm{~N} 22^{\prime} \mathrm{H} 21$ & AGAATAGGGATGAGACAACACAACAGCTTACACTGGGGTA \\
\hline$>\mathrm{M} 40 \mathrm{~N} 2{ }^{\prime} \mathrm{H} 22$ & TATACGTGCTACCGTAAGGGACTGAGACCATACACGGACC \\
\hline$>\mathrm{M} 40 \mathrm{~N} 22^{\prime} \mathrm{H} 24$ & AAGGAAACCACCGGAATGTATGTGGCTGTGCGGCACTAAA \\
\hline$>\mathrm{M} 40 \mathrm{~N} 2{ }^{\prime} \mathrm{H} 26$ & ACGCATAACGATGGAGGAAGGCACGCAGGTGTATGGATGA \\
\hline$>\mathrm{M} 40 \mathrm{~N} 2{ }^{\prime} \mathrm{H} 27$ & TTAGGGGTCAGACGAGAATGTATGAGAAGCACGTAGCCAC \\
\hline$>\mathrm{M} 40 \mathrm{~N} 2{ }^{\prime} \mathrm{H} 28$ & CACGTGCGCAAAGCTATTCGAGTCTCTTATGGGGTGCTAG \\
\hline$>\mathrm{M} 40 \mathrm{~N} 22^{\prime} \mathrm{H} 30$ & AACCAACAAGACCAGCGGGTGCCACTCCAGTCCTACAGCA \\
\hline$>\mathrm{M} 40 \mathrm{~N} 22^{\prime} \mathrm{H} 31$ & GATCAACAGTAGAAAATCGGCAGGTAGGGACACAAACGAA \\
\hline$>\mathrm{M} 40 \mathrm{~N} 22^{\prime} \mathrm{H} 32$ & AATATGACGGCGGCAGCATCGCTCGCCGTCCTCAGTATAC \\
\hline$>\mathrm{M} 40 \mathrm{~N} 2{ }^{\prime} \mathrm{H} 34$ & CCCGGGTATGCGACAGAACTTATGGGGGTTAAGGACGACA \\
\hline$>\mathrm{M} 40 \mathrm{~N} 22^{\prime} \mathrm{H} 35$ & CCCGGGTATGCGACAGAACTTATGGGGGTTAAGGACGACA \\
\hline$>\mathrm{M} 40 \mathrm{~N} 2{ }^{\prime} \mathrm{H} 36$ & ATGCTTTGTTTGAGGGACTTAGGGGTACGTGAGACCGTCT \\
\hline$>\mathrm{M} 40 \mathrm{~N} 22^{\prime} \mathrm{H} 37$ & GGATGTTCCAAGCGGAGGAAGGGCTGTCTCGATCCATCGA \\
\hline$>\mathrm{M} 40 \mathrm{~N} 2{ }^{\prime} \mathrm{H} 38$ & AGTCAAGGAAGAGGGAGCGCGAGCGCGGTGGGGAGTAGCC \\
\hline$>\mathrm{M} 40 \mathrm{~N} 2{ }^{\prime} \mathrm{H} 39$ & TGGCGAAGTACCACATTCGTAGAAAGCACGTAGAGGATGC \\
\hline$>\mathrm{M} 40 \mathrm{~N} 2{ }^{\prime} \mathrm{H} 40$ & GACTGGATACCAGGACAGAGTGTAAGACGGGTAGGAAGCA \\
\hline$>\mathrm{M} 40 \mathrm{~N} 22^{\prime} \mathrm{H} 41$ & AAGGAAGGCCATGGGTTGAAAAGTACCCAATTGTTCGATT \\
\hline$>\mathrm{M} 40 \mathrm{~N} 22^{\prime} \mathrm{H} 42$ & TGCAGCAATAGCGTGTGGGATGAGAGCAAATGCTACGACA \\
\hline$>\mathrm{M} 40 \mathrm{~N} 22^{\prime} \mathrm{H} 43$ & СTGGCGTGGGGGGGGCGGGACTCTACAGACCCAGAGGAAT \\
\hline$>\mathrm{M} 40 \mathrm{~N} 2{ }^{\prime} \mathrm{H} 44$ & ATCAGGAAGGCACACTGAGACCGGGTTGGACTTGCGTGTG \\
\hline$>\mathrm{M} 40 \mathrm{~N} 2{ }^{\prime} \mathrm{H} 48$ & CAGTAGAGGAACAAGCGGCGCAGAGGATCTTTAAGAACTG \\
\hline$>\mathrm{M} 40 \mathrm{~N} 2{ }^{\prime} \mathrm{H} 50$ & TACCGGCCAGCATCTAAAAGTCATGTCGGAGGCTCGTTGG \\
\hline$>\mathrm{M} 4$ ON2 ${ }^{\prime} \mathrm{H} 51$ & ACACGGCAACAGAGTCGGGAAAGGTGCAGCGGCATACAGG \\
\hline$>\mathrm{M} 4$ ON2 ${ }^{\prime} \mathrm{H} 52$ & CGATACAGCTAGCGTGGAAGATGTGGGTATGAAAGCAGAT \\
\hline$>\mathrm{M} 40 \mathrm{~N} 22^{\prime} \mathrm{H} 53$ & GCAGGCCGGGAAAGGACCTTGGGGGATTAGCTGTGCGGAA \\
\hline$>\mathrm{M} 40 \mathrm{~N} 2$ ' H55 & GCCCAAAACACCGCGAAGATTTGCGAAAGGGGGAATGGGT \\
\hline$>\mathrm{M} 40 \mathrm{~N} 2{ }^{\prime} \mathrm{H} 58$ & ATACGGAAGGTAGGGATCCGTCACATGTTAGGGCCTACTT \\
\hline$>\mathrm{M} 40 \mathrm{~N} 2{ }^{\prime} \mathrm{H} 60$ & CTGGTGCAGGTCAGGACAACATTGGGGCAGGTCACGCATA \\
\hline
\end{tabular}




\section{Materials and methods}

Reagent: Unless noted otherwise, all DNA sequences were purchased from Integrated DNA Technologies (IDT) and all other chemical reagent were purchased from Millipore Sigma. DNA concentration were measured through $260 \mathrm{~nm}$ absorbance on a nanodrop (Thermo fisher NanoDrop One/One $e^{c}$ )

\section{Virus propagation/purification}

Methods used for the propagation and purification of the virus particles are detailed in chapter 3.

\section{Selection against MARV GP}

The selection was performed with an ssDNA library with 40 nucleotides random region flanked by two fixed primer binding sites (5'-TAGGGAAGAGAAGGACATATGAT40N- TTGACTAGTACATGACCACTTGA-3') which was synthesized by Integrated DNA Technology (IDT). The 3' primer was annealed to the libraries before every round during the thermal renaturing step in every round. In round one, 1 nmole of ssDNA was annealed to the 3 'primer by heating the sequences at $90^{\circ} \mathrm{C}$ for $3 \mathrm{~min}$ and cooling gradually to room temperature (1.3-fold excess primer was used). The complex was incubated with $6 \times 10^{6} \mathrm{pfu}$ of purified rVSV/MARV_GP for $30 \mathrm{~min}$ at $37^{\circ} \mathrm{C}$ in a SELEX buffer (50 mM Tris $\mathrm{HCl} \mathrm{pH} \mathrm{7.5,} 140 \mathrm{mM} \mathrm{NaCl}, 5 \mathrm{mM} \mathrm{KCl}, 5 \mathrm{mM} \mathrm{CaCl} 2,2 \mathrm{mM} \mathrm{MgCl}_{2}$ ) at a final volume of $100 \mu \mathrm{L}$. The unbound sequences were removed by passing the mixture through $\mathrm{KOH}$ treated nitrocellulose filter (HAWPO2500; Millipore Sigma, St Louis) under vacuum (XX2702550) followed by 3-1mL washes. Bound sequences on 
the nitrocellulose filter were eluted with elution buffer $(8 \mathrm{M}$ urea, $50 \mathrm{mM} \mathrm{NaCl}$, and 10 mM EDTA). Next, ssDNA was ethanol precipitated out and amplified through 15 rounds of PCR. A fraction of the pre-amplified sequences was further amplified through 15 cycles of PCR. ssDNA was generated through a strand separation/purification step. Nitrocellulose and non-target subtractive steps were included in the subsequent rounds until the end of the selection (nitrocellulose filter binders round 2-3 \& rVSV rounds 411). Virus concentration was gradually decreased from round 2 onwards to increase stringency. Also, a final concentration of $1 \mathrm{mg} / \mathrm{ml}$ of salmon sperm DNA was coincubated with the library to increase stringency as well. The parameters used for the selection are summarized in Table S1.

\section{PCR conditions and strand separation.}

List of primers used

Forward Primer: -5' TAGGGAAGAGAAGGACATATGAT 3'

A18-HEG-modified reverse primer:

5' AAAAAAAAAAAAAAAAAAAA-HEG-5' TCAAGTGGTCATGTACTAGTCAA 3' 3' primer: -5' TCAAGTGGTCATGTACTAGTCAA 3'

PCR amplifications were performed with the forward and A18-HEG-modified reverse primer $(0.5 \mu \mathrm{M}$ each final concentration). 15 cycles of thermal recycling were used for the amplification steps using the following thermal cycling conditions: initial denaturing 5 min, cycling (denaturing $95^{\circ} \mathrm{C}, 30 \mathrm{~s}$, annealing $55^{\circ} \mathrm{C}, 30 \mathrm{~s}$, elongation $72^{\circ} \mathrm{C}, 30 \mathrm{~s}$ ), final elongation $72^{\circ} \mathrm{C} 10 \mathrm{~min}$. Single-stranded DNA was generated through a hexaethylene glycol-poly adenine (20 nts) modified reverse primer. A PCR with the A18-HEG- 
modified primer enables us to separate the sense and anti-sense strands through $6 \%$ denaturing Urea Page gel.

\section{Cloning and low throughput sequencing.}

The enriched population was cloned into a linearized pUC19 plasmid vector using Infusion ${ }^{\circledR}$. (plasmid linearize at the $\mathrm{XbaI}$ restriction site). The infusion reaction was transformed into Stellar ${ }^{\mathrm{TM}}$ Competent Cells according to the manufacturer's instructions (Takara, cat\# 636766) and incubated overnight at $37^{\circ} \mathrm{C}$. Individual colonies were propagated in a $5 \mathrm{~mL}$ bacteria growth media with ampicillin. Plasmid DNAs were isolated from the cultures using GenJet Plasmid Miniprep Kit (Thermo Fisher, cat\#K0503). Sanger sequencing was performed utilizing a 3730xl 96-capillary DNA Analyzer with Applied Biosystems Big Dye Terminator cycle sequencing chemistry. (DNA Core facility, LSC University of Missouri).

\section{Filter retention assay.}

To generate radiolabeled sequences, forward primer was labeled at the 5' end and used for the PCR amplification step. The primer was labeled with [ $\gamma$-32P]-ATP (PerkinElmer) by T4-polynucleotide kinase (NEB), according to manufacturer's instruction. Following PCR, we performed strand separation as described above. For the binding assays, $10 \mathrm{nM}$ (1 pmole) radiolabeled ssDNA was annealed to $13 \mathrm{nM}$ of 3'primer and incubated with viral particles in SELEX buffer and allowed to equilibrate for $30 \mathrm{~min}$ at $37^{\circ} \mathrm{C}$. The samples were passed through $\mathrm{KOH}$ treated nitrocellulose filters and immediately washed 3 times with $1 \mathrm{~mL}$ Selex buffer. The filters were air-dried, transferred into a scintillation tube with $3 \mathrm{ml}$ scintillation fluid. Radioactivity retained on the filters was quantified 
using a scintillation counter. Percent ssDNA bound was calculated by determining the fraction of radioactivity on the filter with background filter binding subtracted. For dosedependent filter binding assays, the data were fit to a nonlinear regression curve using Prism 8.

\section{Plasmid and transfections.}

Plasmids.

pCMV3-GP (Lake Victoria Marburgvirus Lake Victoria marburgvirus (MARV) (strainMusoke) glycoprotein / GP (Codon Optimized) natural ORF mammalian expression plasmid (Sino Biological Inc.)

phCMV-GP (Mayinga strain-76 ebolavirus) (Sapphire Lab, Scripps research institute)

For HeLa cell transfection experiments, $5 \times 10^{5}$ cells were plated in 6 well plate the day before transfection. $24 \mathrm{hr}$ after seeding, the cells were transfected with the appropriate plasmid using jetPRIME® transfection reagent. (Polyplus transfection). Transfection was done following manufacture's protocol. Cells are assessed for GP expression, $24 \mathrm{hr}$ post transfection.

\section{Cell binding assay/Flow cytometry.}

For the flow cytometry analysis, sequences were annealed to 5'Cy5-labeled 3' primer. The 3'primer was labeled with cy5 NHS ester (Lumiphore, Hunt Valley, MD, USA) in house. The labeling reaction and analytical evaluation were performed as described 
elsewhere. (cite nature com). We used $0.5 \mu \mathrm{M}$ of sequence and $0.16 \mu \mathrm{M}$ of labeled primer to minimize the background signal associated with free primer. Salmon sperm DNA $(1 \mathrm{mg} / \mathrm{ml})$ was added to the samples before incubation with cells to reduce nonspecific binding. HeLa cells were detached from plate using TrypLE dissociation reagent (Thermo fisher, Waltham, MA).

The samples were incubated with virus GP-expressing HeLa cells for 30 min at room temperature in $100 \mu \mathrm{L}$ of SELEX buffer. Short incubation times and lower temperatures were used to reduce non-specific uptake. Cells were spun down at $450 \mathrm{rcf}$ to removed unbound sequences. Cells were then washed with $500 \mu \mathrm{L}$ of SELEX buffer and fixed in $0.5 \%$ paraformaldehyde at $4{ }^{\circ} \mathrm{C}$ for $5 \mathrm{~min}$. Geometric mean fluorescence values of $\sim 3 \times 10^{4}$ events per sample were determined via flow cytometry (Accuri C6 flow cytometer) and analyzed with FlowJo software (Treestar, Ashland, OR, USA). We gated and analyzed viable cells while excluding dead cells through forward and side scatter plots.

\section{MST}

The MST data was obtained using the Monolith NT.115 Pico device with nano-blue and pico-red detection channels (Nano Temper Technologies, Munich, Germany). Cy5-labeled aptamers were thermally renatured in SELEX buffer. Viral particles were incubated at room temperature in $40 \mu \mathrm{L}$ of $50 \mathrm{nM}$ aptamer samples in a SELEX buffer. We added $0.05 \%$ tween 20 to minimize virus aggregation. After $1 \mathrm{hr}$ incubation, MST-grade standard capillaries were filled with the samples (about $10 \mu \mathrm{L}$ of samples). The samples were 
analyzed using red-LED MST power at 20\% and the time window set to $5 \mathrm{~s}$ before IR laser, 30 s during IR laser, and $5 \mathrm{~s}$ after IR laser. The temperature was set too $25^{\circ} \mathrm{C}$ for all measurements. Fluorescent intensity changes $(\mathrm{ex}=620 \mathrm{~nm} \pm 25 \mathrm{~nm}$ and fluorescence emission were detected at em $=690 \mathrm{~nm} \pm 30 \mathrm{~nm}$ ), were calculated between IR start time (Fcold) and $15 \mathrm{~s}$ into IR end time (Fhot). We calculated the normalized fluorescence $\Delta \mathrm{F}_{\text {norm }}$ $\left.\left(\Delta \mathrm{F}_{\text {norm }}=\mathrm{F}_{\text {hot }} \mathrm{F}_{\text {cold }}\right) \times 10^{3}\right)$ and plotted it against virus particles. To calculate fraction bound, all $\Delta \mathrm{F}_{\text {norm }}$ values were divided by the curve amplitude. The data were fitted to a curve using Prism software. (the equation of the curve, $Y=$ Bottom $+($ Top-Bottom $) /\left(1+10^{\wedge}((\operatorname{LogIC50}-\right.$ X) ${ }^{*}$ HillSlope), where Span $=$ Top-Bottom $)$.

\section{Gel shift assays.}

One picomole of cy5-labeled aptamer or control sequence were incubated with viral particles in $20 \mu \mathrm{L}$ final volume for $1 \mathrm{hr}$ at room temperature. The samples were loaded onto $0.2 \%$ TAE agarose gel. Gel electrophoresis was done at $50 \mathrm{~W}$ in $4^{\circ} \mathrm{C}$ for $1.5 \mathrm{hr}$. The gel was then imaged using a Typhoon FLA9000 (GE Healthcare) with Cy5 settings (emission $665 \mathrm{~nm}$, excitation $635 \mathrm{~nm}$ ).

\section{ELONA}

We performed ELONA using a protocol described elsewhere with slight modifications. Briefly, ELISA plates were coated mouse anti-MARV GP mAb (5C1) (Catalog \#: 0203023, IBT Bioservices) by incubating $100 \mu \mathrm{L} 4 \mu \mathrm{g} / \mathrm{ml}$ antibody in the wells overnight at $4^{\circ} \mathrm{C}$ in a coating buffer $\left(0.03 \mathrm{M} \mathrm{NaCO}_{3}, 0.068 \mathrm{NaHCO}_{3} \mathrm{pH} 9.4\right)$. The next day, the wells were washed three times with wash buffer (PBS, $2 \mathrm{mM} \mathrm{MgCl}, 0.05 \%$ Tween20 $\mathrm{pH}$ 7.5) and blocked by adding $400 \mu \mathrm{L}$ blocking buffer (PBS, 1\% BSA, 1\% dextran sulfate, 6.5 - 
$10 \mathrm{kDa}$ ) and incubating at room temperature for $2 \mathrm{hr}$. The virus particles were captured by the immobilized antibodies by incubating the particles $(100 \mu \mathrm{L})$ total volume in the wells at $37^{\circ} \mathrm{C}$ for $2 \mathrm{hr}$. The wells were washed times with wash buffer and incubated with biotinylated aptamers. The aptamers were biotinylated by annealing a 5'-biotinylated 3' primer to the pbs as described above. (using $1 \mu \mathrm{M}$ of aptamer and $0.5 \mu \mathrm{M}$ of biotinylated primer, the final volume is $100 \mu \mathrm{L}$ ). Next unbound aptamer was removed by washing the wells 3 times with selection buffer. Streptavidin-tagged Horseradish peroxidase (R\&D Systems Catalog\#: DY998) was added to each well and incubated at $37^{\circ} \mathrm{C}$ for $30 \mathrm{~min}$. The plates were washed five times and developed using substrate tetramethylbenzidine (TMB) (BD Biosciences, catalog\#: 555214) at room temperature for 15-20 min. The reaction was stopped by adding $2 \mathrm{~N}$ hydro-sulfuric acid $\left(2 \mathrm{~N} \mathrm{H}_{2} \mathrm{SO}_{4}\right)$. We measured the optical densities at $450 \mathrm{~nm}$ using an EnSpire Multimode plate reader (PerkinElmer). The optical density values at $450 \mathrm{~nm}$ were plotted against the number of virus particles. 


\section{Reference}

(1) Geisbert, T. W.; Daddario-DiCaprio, K. M.; Geisbert, J. B.; Young, H. A.; Formenty, P.; Fritz, E. A.; Larsen, T.; Hensley, L. E. Marburg Virus Angola Infection of Rhesus Macaques: Pathogenesis and Treatment with Recombinant Nematode Anticoagulant Protein C2. J. Infect. Dis. 2007, 196 (s2), S372-S381. https://doi.org/10.1086/520608.

(2) Towner, J. S.; Khristova, M. L.; Sealy, T. K.; Vincent, M. J.; Erickson, B. R.; Bawiec, D. A.; Hartman, A. L.; Comer, J. A.; Zaki, S. R.; Ströher, U.; Gomes da Silva, F.; del Castillo, F.; Rollin, P. E.; Ksiazek, T. G.; Nichol, S. T. Marburgvirus Genomics and Association with a Large Hemorrhagic Fever Outbreak in Angola. J. Virol. 2006, 80 (13), 6497-6516. https://doi.org/10.1128/jvi.00069-06.

(3) Jacob, S. T.; Crozier, I.; Fischer, W. A.; Hewlett, A.; Kraft, C. S.; Vega, M. A. de La; Soka, M. J.; Wahl, V.; Griffiths, A.; Bollinger, L.; Kuhn, J. H. Ebola Virus Disease. Nature Reviews Disease Primers. Nature Research December 1, 2020. https://doi.org/10.1038/s41572-020-0147-3.

(4) Szeitner, Z.; András, J.; Gyurcsányi, R. E.; Mészáros, T. Is Less More? Lessons from Aptamer Selection Strategies. J. Pharm. Biomed. Anal. 2014, 101, 58-65. https://doi.org/10.1016/J.JPBA.2014.04.018.

(5) Malherbe, H.; Strickland-Cholmley, M. Human Disease from Monkeys (Marburg Virus). Lancet 1968, 1 (7557), 1434. https://doi.org/10.1016/S01406736(68)92023-0.

(6) Siegert, R.; Shu, H. L.; Slenczka, W. Isolierung Und Identifizierung Des „Marburg-Virus”. Dtsch. Medizinische Wochenschrift 1968, 93 (12), 604-612. 
https://doi.org/10.1055/s-0028-1105103.

(7) Martini, G. A.; Knauff, H. G.; Schmidt, H. A.; Mayer, G.; Baltzer, G. A Hitherto Unknown Infectious Disease Contracted from Monkeys. "Marburg-Virus"

Disease. Ger. Med. Mon. 1968, 13 (10), 457-470.

(8) Andrew Flyak, A. I.; Ilinykh, P. A.; Bukreyev, A.; Crowe, J. E.; Correspondence, J. Mechanism of Human Antibody-Mediated Neutralization of Marburg Virus.

2015. https://doi.org/10.1016/j.cell.2015.01.031.

(9) Hashiguchi, T.; Fusco, M. L.; Bornholdt, Z. A.; Lee, J. E.; Flyak, A. I.; Matsuoka, R.; Kohda, D.; Yanagi, Y.; Hammel, M.; Crowe, J. E.; Saphire, E. O. Structural Basis for Marburg Virus Neutralization by a Cross-Reactive Human Antibody. Cell 2015, 160 (5), 904-912. https://doi.org/10.1016/j.cell.2015.01.041.

(10) Schnell, M. J.; Buonocore, L.; Kretzschmar, E.; Johnson, E.; Rose, J. K. Foreign Glycoproteins Expressed from Recombinant Vesicular Stomatitis Viruses Are Incorporated Efficiently into Virus Particles. Proc. Natl. Acad. Sci. U. S. A. 1996, 93 (21), 11359-11365. https://doi.org/10.1073/pnas.93.21.11359.

(11) Anantpadma, M.; Kouznetsova, J.; Wang, H.; Huang, R.; Kolokoltsov, A.; Guha, R.; Lindstrom, A. R.; Shtanko, O.; Simeonov, A.; Maloney, D. J.; Maury, W.; LaCount, D. J.; Jadhav, A.; Davey, R. A. Large-Scale Screening and Identification of Novel Ebola Virus and Marburg Virus Entry Inhibitors. Antimicrob. Agents Chemother. 2016, 60 (8), 4471-4481. https://doi.org/10.1128/AAC.00543-16.

(12) Mulangu, S.; Dodd, L. E.; Davey, R. T.; Tshiani Mbaya, O.; Proschan, M.; Mukadi, D.; Lusakibanza Manzo, M.; Nzolo, D.; Tshomba Oloma, A.; Ibanda, A.; Ali, R.; Coulibaly, S.; Levine, A. C.; Grais, R.; Diaz, J.; Lane, H. C.; Muyembe- 
Tamfum, J.-J.; the PALM Writing Group. A Randomized, Controlled Trial of Ebola Virus Disease Therapeutics. N. Engl. J. Med. 2019, 381 (24), 2293-2303. https://doi.org/10.1056/NEJMoa1910993.

(13) Lucey, D. R. New Treatments for Ebola Virus Disease. The BMJ. BMJ Publishing Group September 6, 2019. https://doi.org/10.1136/bmj.15371.

(14) Misasi, J.; Gilman, M. S. A.; Kanekiyo, M.; Gui, M.; Cagigi, A.; Mulangu, S.; Corti, D.; Ledgerwood, J. E.; Lanzavecchia, A.; Cunningham, J.; MuyembeTamfun, J. J.; Baxa, U.; Graham, B. S.; Xiang, Y.; Sullivan, N. J.; McLellan, J. S. Structural and Molecular Basis for Ebola Virus Neutralization by Protective Human Antibodies. Science 2016, 6117 (February), 1343-1346. https://doi.org/10.1126/science.aad6117.

(15) Geisbert, T.; Daddario-Dicaprio, K.; Lewis, M.; al., et. Vesicular Stomatitis VirusBased Ebola Vaccine Is Well-Tolerated and Protects Immunocompromised NonHuman Primates. PLoS Pathog 2008, 4, e1000225.

(16) Geisbert, T. W. First Ebola Virus Vaccine to Protect Human Beings? The Lancet. Lancet Publishing Group February 4, 2017, pp 479-480. https://doi.org/10.1016/S0140-6736(16)32618-6.

(17) First Ebola Vaccine Approved. Nature biotechnology. NLM (Medline) January 1, 2020, p 6. https://doi.org/10.1038/s41587-019-0385-7.

(18) Smith, K. F.; Goldberg, M.; Rosenthal, S.; Carlson, L.; Chen, J.; Chen, C.; Ramachandran, S. Global Rise in Human Infectious Disease Outbreaks. J. R. Soc. Interface 2014, 11 (101), 20140950. https://doi.org/10.1098/rsif.2014.0950.

(19) Martines, R. B.; Ng, D. L.; Greer, P. W.; Rollin, P. E.; Zaki, S. R. Tissue and 
Cellular Tropism, Pathology and Pathogenesis of Ebola and Marburg Viruses. $J$. Pathol. 2015, 235 (2), 153-174. https://doi.org/10.1002/path.4456.

(20) Leroy, E. M.; Baize, S.; Lu, C. Y.; McCormick, J. B.; Georges, A. J.; GeorgesCourbot, M. C.; Lansoud-Soukate, J.; Fisher-Hoch, S. P. Diagnosis of Ebola Haemorrhagic Fever by RT-PCR in an Epidemic Setting. J. Med. Virol. 2000, 60 (4), 463-467. https://doi.org/10.1002/(SICI)1096-9071(200004)60:4<463::AIDJMV15>3.0.CO;2-M.

(21) Murray, M. J. Ebola Virus Disease: A Review of Its Past and Present. Anesthesia and Analgesia. Lippincott Williams and Wilkins September 26, 2015, pp 798-809. https://doi.org/10.1213/ANE.0000000000000866.

(22) Zhou, J.; Rossi, J. Aptamers as Targeted Therapeutics: Current Potential and Challenges. Nat. Rev. Drug Discov. 2016, 16 (3), 181-202. https://doi.org/10.1038/nrd.2016.199.

(23) Baker, M. Blame It on the Antibodies. Nature 2015, 521 (7552), 274-276. https://doi.org/10.1038/521274a.

(24) Feldmann, H.; Volchkov, V. E.; Volchkova, V. A.; Ströher, U.; Klenk, H. D. Biosynthesis and Role of Filoviral Glycoproteins. Journal of General Virology. Society for General Microbiology December 1, 2001, pp 2839-2848. https://doi.org/10.1099/0022-1317-82-12-2839.

(25) Deng, B.; Lin, Y.; Wang, C.; Li, F.; Wang, Z.; Zhang, H.; Li, X. F.; Le, X. C. Aptamer Binding Assays for Proteins: The Thrombin Example-A Review. Analytica Chimica Acta. Elsevier July 21, 2014, pp 1-15. https://doi.org/10.1016/j.aca.2014.04.055. 
(26) van den Kieboom, C. H.; van der Beek, S. L.; Mészáros, T.; Gyurcsányi, R. E.; Ferwerda, G.; de Jonge, M. I. Aptasensors for Viral Diagnostics. TrAC - Trends in Analytical Chemistry. Elsevier B.V. December 1, 2015, pp 58-67. https://doi.org/10.1016/j.trac.2015.05.012.

(27) Tuerk, C.; Gold, L. Systematic Evolution of Ligands by Exponential Enrichment: RNA Ligands to Bacteriophage T4 DNA Polymerase. Science 1990, 249 (4968), $505-510$.

(28) Ellington, A. D.; Szostak, J. W. In Vitro Selection of RNA Molecules That Bind Specific Ligands. Nature 1990, 346 (6287).

(29) Burke, D. H.; Gold, L. RNA Aptamers to the Adenosine Moiety of S-Adenosyl Methionine: Structural Inferences from Variations on a Theme and the Reproducibility of SELEX; Oxford University Press, 1997; Vol. 25.

(30) Zhou, J.; Rossi, J. Aptamers as Targeted Therapeutics: Current Potential and Challenges. Nat. Rev. Drug Discov. 2016. https://doi.org/10.1038/nrd.2016.199.

(31) Hicke, B. J.; Marion, C.; Chang, Y. F.; Gould, T.; Lynott, C. K.; Parma, D.; Schmidt, P. G.; Warren, S. Tenascin-C Aptamers Are Generated Using Tumor Cells and Purified Protein. J. Biol. Chem. 2001, 276 (52), 48644-48654. https://doi.org/10.1074/jbc.M104651200.

(32) Sefah, K.; Shangguan, D.; Xiong, X.; O’Donoghue, M. B.; Tan, W. Development of DNA Aptamers Using Cell-Selex. Nat. Protoc. 2010, 5 (6), 1169-1185. https://doi.org/10.1038/nprot.2010.66.

(33) Renders, M.; Miller, E.; Lam, C. H.; Perrin, D. M. Whole Cell-SELEX of Aptamers with a Tyrosine-like Side Chain against Live Bacteria. Org. Biomol. 
Chem. 2017, 15 (9), 1980-1989. https://doi.org/10.1039/C6OB02451C.

(34) Pan, W.; Craven, R. C.; Qiu, Q.; Wilson, C. B.; Wills, J. W.; Golovine, S.; Wang, J. F. Isolation of Virus-Neutralizing RNAs from a Large Pool of Random Sequences. Proc. Natl. Acad. Sci. U. S. A. 1995, 92 (25), 11509-11513. https://doi.org/10.1073/pnas.92.25.11509.

(35) Narayan, C.; Kwon, J.; Kim, C.; Kim, S. J.; Jang, S. K. Virus-Based SELEX (Viro-SELEX) Allows Development of Aptamers Targeting Knotty Proteins. Analyst 2020, 145 (4), 1473-1482. https://doi.org/10.1039/c9an01943j.

(36) Shubham, S.; Hoinka, J.; Banerjee, S.; Swanson, E.; Dillard, J. A.; Lennemann, N. J.; Przytycka, T. M.; Maury, W.; Nilsen-Hamilton, M. A 2'FY-RNA Motif Defines an Aptamer for Ebolavirus Secreted Protein. Sci. Rep. 2018, 8 (1). https://doi.org/10.1038/s41598-018-30590-8.

(37) Liu, Y.; Kuan, C. T.; Mi, J.; Zhang, X.; Clary, B. M.; Bigner, D. D.; Sullenger, B. A. Aptamers Selected against the Unglycosylated EGFRvIII Ectodomain and Delivered Intracellularly Reduce Membrane-Bound EGFRvIII and Induce Apoptosis. Biol. Chem. 2009, 390 (2), 137-144.

https://doi.org/10.1515/BC.2009.022.

(38) Ilinykh, P. A.; Shen, X.; Flyak, A. I.; Kuzmina, N.; Ksiazek, T. G.; Crowe Jr., J. E.; Bukreyev, A. Chimeric Filoviruses for Identification and Characterization of Monoclonal Antibodies. J Virol 2016. https://doi.org/10.1128/JVI.00101-16.

(39) Drolet, D. W.; Moon-McDermott, L.; Romig, T. S. An Enzyme-Linked Oligonucleotide Assay. Nat. Biotechnol. 1996, 14 (8), 1021-1025. https://doi.org/10.1038/nbt0896-1021. 
(40) Mallikaratchy, P. Evolution of Complex Target SELEX to Identify Aptamers against Mammalian Cell-Surface Antigens. Molecules. MDPI AG February 1, 2017, p 215. https://doi.org/10.3390/molecules22020215.

(41) Wong, I.; Lohman, T. M. A Double-Filter Method for Nitrocellulose-Filter Binding: Application to Protein-Nucleic Acid Interactions (DNA-Protein Interactions/Equilibrium Binding); 1993; Vol. 90.

(42) Bar, S.; Takada, A.; Kawaoka, Y.; Alizon, M. Detection of Cell-Cell Fusion Mediated by Ebola Virus Glycoproteins. J. Virol. 2006, 80 (6), 2815-2822. https://doi.org/10.1128/jvi.80.6.2815-2822.2006. 


\title{
Chapter 5: Hybrid SELEX Generates Ebola Virus
}

\section{Glycoprotein targeting aptamers}

This work will be part of the material that will be submitted for publication after further developments outlined at the end of the chapter. Anticipated co-authors include Kwaku D. Tawiah, Amanda Paz, and Donald H. Burke

\begin{abstract}
Ebola virus is a highly virulent pathogen. Promising therapeutic interventions include a recently approved vaccine and antibody-based drugs; however, there is a need for additional therapeutic approaches to avoid the potential emergence of treatment resistant strains. EBOV displays a glycoprotein (GP) on its surface that has proven to be an effective target for therapeutic and diagnostic development. However, antibodies (Abs) are unable to access crucial epitopes required for virus entry due to GP architecture and the virus entry mechanism. Aptamers are small, structured nucleic acid ligands generated through an in vitro combinatorial selection process. Aptamers that bind to EBOV surfaces may block EBOV infection or serve as affinity probes for the development of EBOV biosensors. When generated to have affinity for purified recombinant virus glycoproteins, aptamers may not interact with the glycoprotein in its native context due to conformational and structural differences that may exist between native proteins and the purified variants. Here, we exploited a hybrid SELEX approach that combines cellSELEX for aptamer probes that bind to EBOV GP displayed on HeLa cells, and bead-
\end{abstract}


based SELEX on EBOV GP displayed on virus particles. This approach identified an enriched population that binds to EBOV GP displayed on cells and on viruses.

\section{Introduction}

Ebola virus (EBOV) is a species of filovirus known to infect humans, non-human primates, bats, and other mammals. EBOV and other filoviruses cause severe hemorrhagic fever diseases in humans. Outbreaks of Ebola virus disease (EVD), including recent ones in West Africa and the Democratic Republic of Congo, are characterized by high case mortality rates $(\sim 40-90 \%)^{1}$. Several therapeutic interventions are in the pipeline, including a recently approved vaccine and two antibody cocktail drugs that target the glycoprotein (GP) of EBOV (REGN-EB3 and mAb114) ${ }^{2-6}$. The vaccine is a replication-competent vesicular stomatitis virus engineered to display EBOV GP (rVSV-ZEBOV), thus eliciting antibodies that bind to EBOV GP. Despite these advancements in treatment modalities, there is a need for more therapeutic approaches to support the current ones and to slow down the possible emergence of drug resistance strains due to over-reliance on the limited number of treatment options.

EBOV displays a virally encoded trimeric glycoprotein on its surface, which has proven to be a major target for therapeutics, vaccines, and diagnostics development ${ }^{7}$. EBOV GP is the only virus-encoded protein on the virus surface and is responsible for cellular tropism and virus entry by binding to host cell attachment factors ${ }^{8-11}$, as described in chapter 1. Following attachment, the virus is endocytosed through micropinocytosis, where endosomal maturation triggers GP cleavage by host cathepsins to expose the receptor binding domain $(\mathrm{RBD})^{12}$. The cleaved GP binds to its endosomal receptor, 
Niemann-Pick $\mathrm{C} 1$ protein (NPC1), to trigger membrane fusion and cytosolic delivery ${ }^{13}$. Although GP targeting monoclonal antibodies show great promise as therapeutic options $^{6,14}$, the pre-cleaved GP has a heavily glycosylated mucin-like domain that blocks access of larger molecules such as antibodies (Abs) $(50-150 \mathrm{kDa})$ to crucial neutralizing GP surface, including the NPC1 binding site ${ }^{12,15,16}$. Smaller molecules such as aptamers $(10-30 \mathrm{kDa})$ could potentially bind to neutralizing surfaces that are otherwise inaccessible to Abs.

Aptamers are small structured nucleic acid ligands generated through an in vitro selection process called SELEX ( Systematic Evolution of Ligands by Exponential enrichment) ${ }^{17,18}$. They bind to their molecular targets (such as small molecule, protein, viruses, etc.) with strong affinity and specificity, comparable to or better than those of antibodies and antibody derivatives ${ }^{19,20}$. Aptamers have been developed to bind and in some cases neutralize enveloped viruses such as such as human cytomegalovirus (hCMV) ${ }^{21}$, influenza virus ${ }^{22,23}$, rabiesvirus ${ }^{24}, \mathrm{HIV}-\mathrm{1}^{25-27}$, and Herpes simplex virus ${ }^{28}$. Previous work has generated aptamers that bind to GP from MARV displayed on chimeric particles (rVSV/MARV_GP) and on HeLa cells (Chapter 4). Recently, 2' F- pyrimidine RNA aptamers selected to bind to the secreted glycoprotein (sGP) from EBOLA was reported. Aptamers selected to bind EBOV GP may block the virus entry or be used as tools for epitope discovery. Also, aptamers that bind to EBOV GP could be used to develop simple, low-cost, point-of-care diagnostic probes. 
Off-target binding that occur during aptamer selections can decrease the efficiency to generate aptamers that bind to complex target such as virus particles or whole cells. Different SELEX platforms have distinct sets of non-specific background binding and present different sets off-target binding. Alternating between SELEX platforms can prevent the enrichment of off-target binding sequences and facilitate the enrichment target-specific aptamer. We sought to identify EBOV GP targeting aptamers by utilizing a hybrid SELEX platform, which combines cell SELEX and bead-capture SELEX. The hybrid SELEX platform switches the target environment during the selection. Notably, both platforms utilize natively displayed GP from EBOV as the selection targets. After six rounds of cell SELEX with EBOV GP displayed on HeLa cells and three rounds on bead-immobilized rVSV/EBOV_GP, the evolved population was evaluated for target binding following and was shown to bind to EBOV GP displayed on HeLa cells and on viruses. Also, the evolved population bind to MARV GP when displayed on HeLa cells. Despite three selection rounds with GP displayed chimeric virus particles, the population was shown to have an affinity for HeLa surfaces as well.

\section{Results and discussion}

Cell SELEX was performed to generate aptamers that bind to EBOV GP displayed on HeLa cells. To generate EBOV GP-expressing cells, HeLa cells were transiently transfected with EBOV GP plasmid. The expression level of EBOV GP was evaluated through immunofluorescence flow cytometry $24 \mathrm{hr}$ post transfection. The cells were incubated with EBOV GP- specific monoclonal antibody (KZ52), followed by incubation with a fluorescently tagged secondary antibody. HeLa cells that were transfected with 
EBOV GP plasmid showed higher fluorescent signal ( $\sim$-fold), compared to cells that were transfected with pcDNA3.1 plasmid. When KZ52 is absent, the EBOV GPtransfected cells showed the same low fluorescence as cells transfected with pUC19 (Chapter 4, Fig S4.2). Together, these results provided evidence of EBOV GP expression on the surface of HeLa cells transfected with EBOV plasmid.

The selection utilized an ssDNA library with a $40 \mathrm{nt}$ random region flanked by fixed sequences for primer binding and amplification (5' pbs \& $\left.3^{\prime} \mathrm{pbs}\right)$. As described in chapter 4, the 3'pbs of the library were annealed to a complementary oligodeoxynucleotide $(\mathrm{ODN})$ to minimize the role of the 3'pbs on formation of functional structures. Cell SELEX was performed as previously described ${ }^{29}$. Briefly, the thermal-renatured ssDNA library was incubated with EBOV GP expressing-HeLa cells at $4^{\circ} \mathrm{C}($ Fig 5.1). The ssDNA extraction approach used to isolate target-specific binding sequences during cell SELEX is not capable of separating internalized sequences from cell surface-bound ones. Therefore, performing the selection at lower temperatures prevents sequence internalization by blocking cellular internalization, which is an ATP-dependent processes. Subtractive steps were performed on HeLa cells transfected with pcDNA3.1 (non-GP expressing plasmid) in each round. 


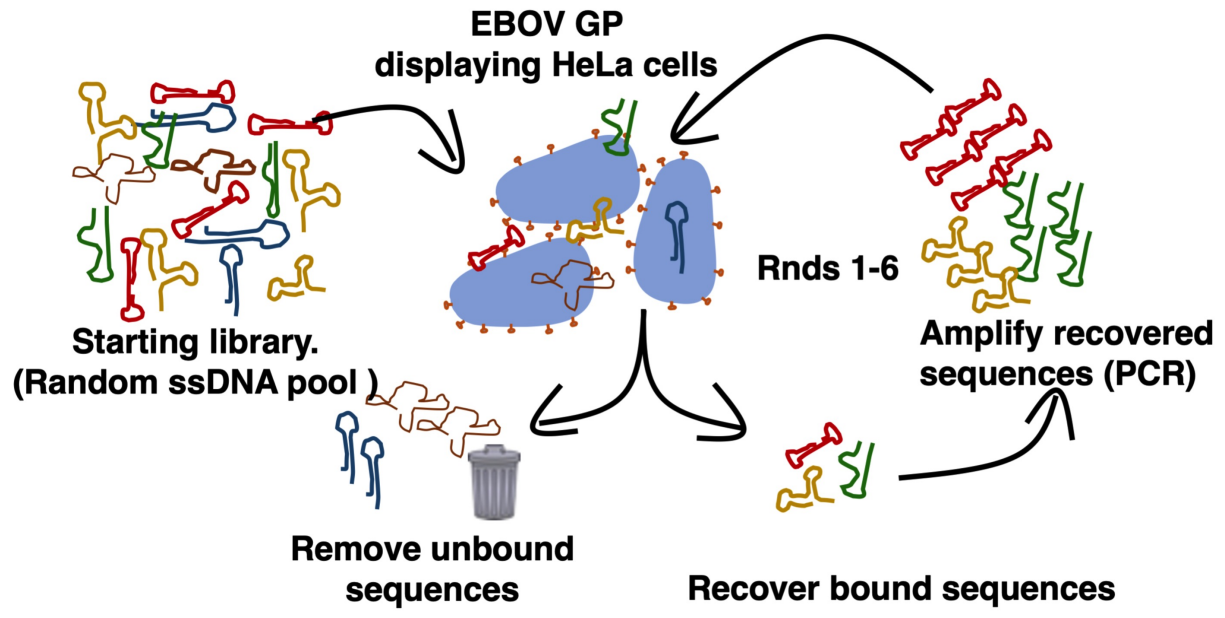

Figure 5.1. Overview of the cell-SELEX phase of the Hybrid SELEX process. In each selection cycle, the ssDNA was incubated with EBOV GP-expressing HeLa cells; unbound ssDNA sequences were washed away, and bound sequences were extracted and amplified. 
Following the sixth round of cell SELEX, flow cytometry was used to evaluate the binding of the round 5 and 6 populations (R5 \& R6) to EBOV GP-expressing HeLa cells relative to the starting library. The libraries were annealed to a fluorescently tagged ODN, then incubated with HeLa cells transfected with EBOV GP. Both R5 and R6 populations showed a slightly higher fluorescent signals on HeLa cells transfected with EBOV GP, compared to their signal on cells that were transfected with pcDNA3.1 plasmid (R5 = 1.3-fold, R6 =1.5-fold) (Fig 5.2). The starting library showed lower fluorescent signal on both cells. Binding specificities of the libraries were further evaluated on MARV GP-expressing HeLa cells. The R5 population showed a higher ( 2fold) fluorescent signal on MARV GP expressing HeLa cells compared to EBOV GPexpressing cells, while the R6 population showed essentially the same signal on both EBOV and MARV GP-expressing HeLa cells (Fig 5.2). These results suggest that the selection enriched sequences that bind to GPs of both EBOV and MARV displayed on HeLa cells. Alternatively, the evolved population could recognize other HeLa surface displayed molecules. The evolved libraries (R 5 \& R6) showed higher fluorescent signal compared to the starting library in all the cells that were evaluated, which suggest the enriched sequence bind to filovirus glycoproteins however, there might be sequences present in the population that have affinity for HeLa surfaces (off-target binders) as well (Fig 5.2). 

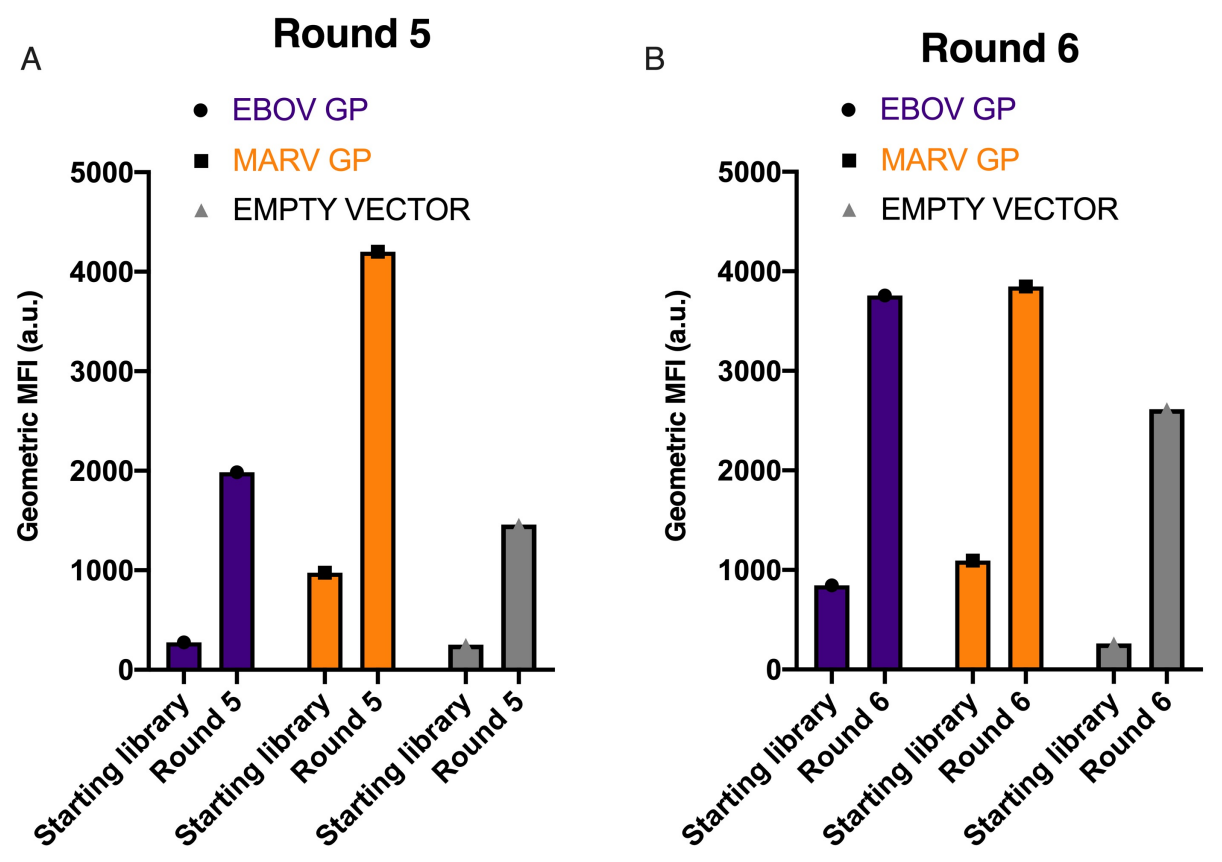

Figure 5.2. Flow cytometry was used to assess binding of the rounds 5 and 6 populations (selected to bind HeLa cells transfected with EBOV GP. A. The round 5 and $\mathbf{B}$. round 6 populations were annealed to fluorescently tagged ODN, then incubated with HeLa cells transfected with plasmids that expressed EBOV GP (purple), MARV GP (orange), or with pcDNA3.1 (gray). Both flow experiments were performed once with $3 \times 10^{4}$ cells count $(\mathrm{n}=1)$. 
Surfaces of virus particles represent a less complex environment compared to HeLa cells. EBOV GP-expressing HeLa cells also display many other proteins on their surfaces. Virus particles such as rVSV/EBOV_GP display mainly the virus encoded GP. Therefore, the sets of off-target binding present on virus surfaces are different from these present on the surfaces of HeLa cells. We reasoned that performing additional rounds of selection on EBOV GP displayed on virus particles would further enrich for sequences that have affinity for EBOV GP and deplete off-target binding sequences. Also, the use of virus displayed GP maintains the target in a native form.

Using ssDNA from the round 6 population, three additional rounds of selection were performed using bead-immobilized rVSV/EBOV_GP particles as selection targets. First, an EBOV GP-specific monoclonal antibody (KZ52) was captured on paramagnetic, Protein-G-coated Dyna-beads. Then to immobilize the virus particles, these beads were incubated with rVSV/EBOV_GP virus particles (Fig 5.3A). In each round of selection, thermally renatured ssDNA was incubated with bead-immobilized virus particles. The beads were collected with a magnet to separate the target-bound sequences from the nonbinding sequences (Fig 5.3B). To deplete sequences that bind to protein $\mathrm{G}$ or KZ52, subtractive steps were performed against the KZ52 coated Protein-G-Dyna-beads (no immobilized virus) before every positive round. Also, the stringency was increased over time by decreasing the amount of immobilized virus particle in the second and third cycles. 
A

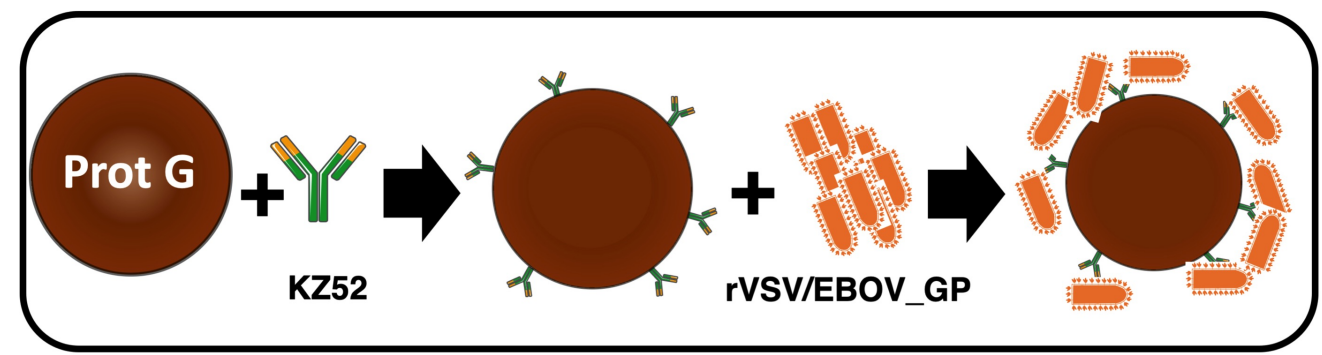

B

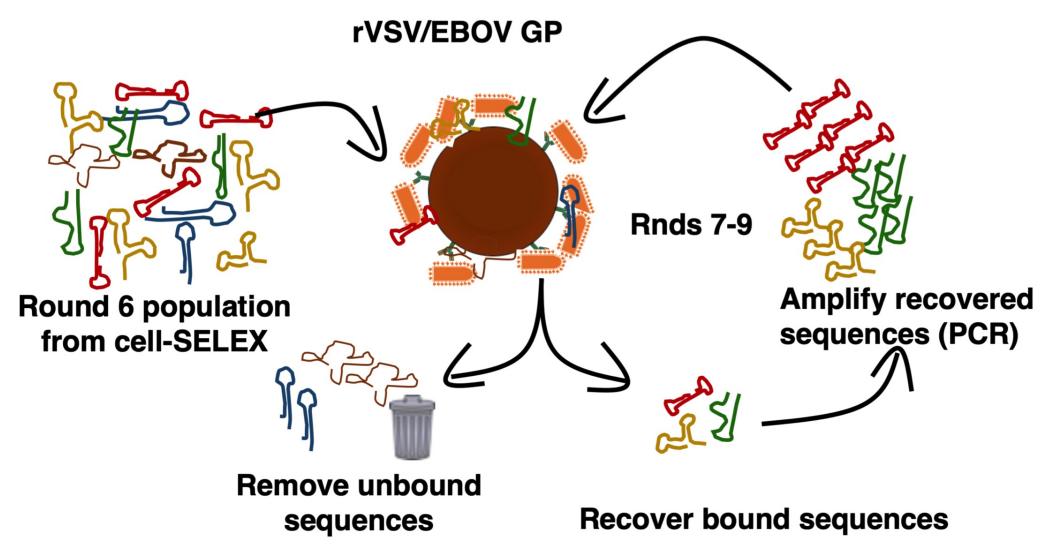

Figure 5.3. Overview of the bead-based virus capture and SELEX process. A. The surfaces of protein G Dyna beads were coated with KZ52 (EBOV GP-specific monoclonal antibody) and used to capture rVSV/EBOV_GP particles. B. The captured virus particles were used as selection targets. The evolved library from R6 (cell-SELEX) were incubated with the virus particles captured on the Dynabeads; bound sequences were captured using a magnet. 
The R7 and R9 evolved libraries were evaluated for binding to EBOV GP displayed HeLa cells. First, ssDNA from the R7 population (6 rounds on cells \& 1 round on immobilized virus particles) and from the starting library were annealed to fluorescently tagged ODN and their binding to the target (EBOV GP displayed on HeLa cells) and non-target targets (MARV GP displayed on HeLa cell and HeLa cells transfected with pcDNA3.1) were assessed through flow cytometry. The round 7 population shows $\sim 2$ to 3-fold fluorescent signal over the starting library when assessed on HeLa cells transfected with EBOV GP, MARV GP, or pcDNA3.1. However, the fluorescent signal from the round 7 population is $\sim 2$-fold higher in cells that display EBOV GP and MARV GP, compared to cells transfected with pcDNA3.1 (Fig 5.4). Similar to the round 6 population, the evolved library differentially binds to HeLa cells transfected either EBOV or MARV GP and HeLa cells transfected with pcDNA3.1. 


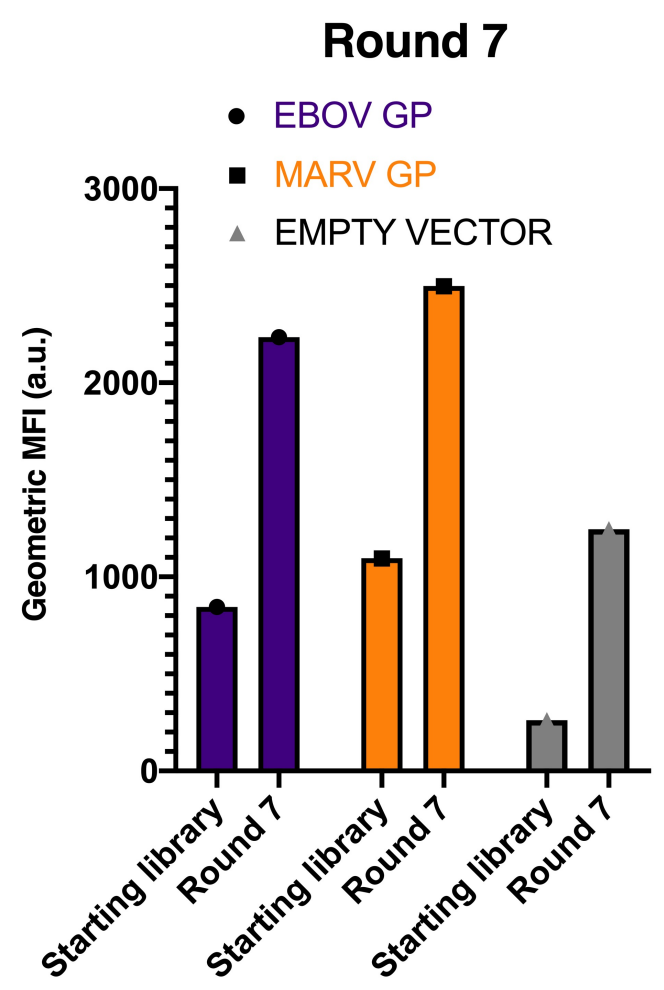

Figure 5.4. Flow cytometry was used to assess binding of the rounds (6 rounds on EBOV GP-expressing HeLa cells, 1 round on bead-captured rVSV/EBOV_GP). The evolved population was annealed to fluorescently tagged ODN, then incubated with HeLa cells transfected with plasmids that expressed EBOV GP (purple), MARV GP (orange), or with pcDNA3.1 (gray). (n 1) 
A more comprehensive analysis of target binding was performed on the R9 population. First, radiolabeled R9 population and starting library were evaluated for dose-dependent binding to purified rVSV/EBOV_GP particles in a filter retention assay. The round 9 population shows a strong dose-dependent signal relative to the starting library for binding to rVSV/EBOV_GP (Fig 5.5A). Second, the radiolabeled libraries were evaluated for binding to rVSV/EBOV_GP particles immobilized on beads surfaces. Again, the round 9 population showed higher radioactivity signal when assessed on immobilized virus, relative to the starting library (3-fold binding over starting library) (Fig 5.5B). Finally, flow cytometry was used to assess the binding of the round 9 population to EBOV GP displayed on HeLa cells. Both the starting library and the round 9 population were annealed to fluorescently tagged ODN and evaluated for binding to HeLa cells that were transfected with EBOV GP, MARV GP or pcDNA3.1. The round 9 population (selected to bind to EBOV GP) shows a slightly higher (1.5-fold) fluorescent signal on cells transfected with either EBOV GP or MARV GP compared to the starting library. Binding assessment on cells transfected with pcDNA3.1 show that the evolved population has a slightly higher, but perhaps insignificant (1.2-fold) fluorescent signal, compared to the starting library (Fig 5.5C). Both the radiolabeled filter retention assay and bead-capture binding assays show that the round 9 population binds to rVSV/EBOV_GP. While the flow cytometry assay confirms the binding of the round 9 population to the EBOV GP, it also shows that the round 9 population also has affinity for non-EBOV GP expressing HeLa surfaces. 


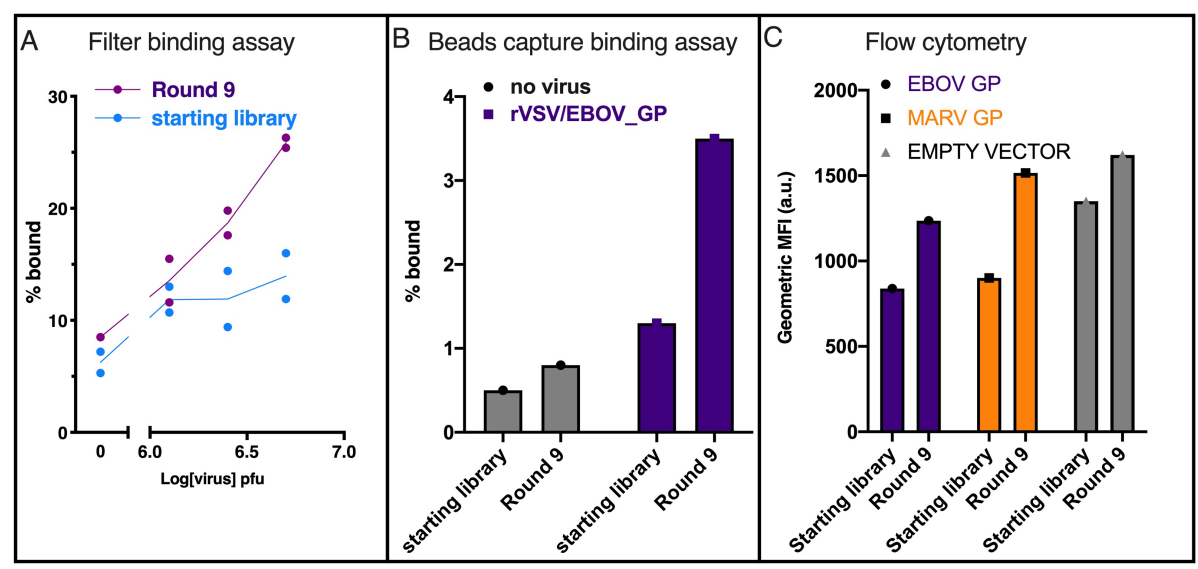

Figure 5.5. Comprehensive enrichment analysis of the evolved library selected for binding to EBOV GP. Two radiolabeled ssDNA input libraries (round 9 and the starting population) were evaluated for binding to purified target (rVSV/EBOV_GP), using different binding assay approaches A. In filter binding assay, round 9 population shows strong dose-dependent signal relative to the starting library $(n=2)$. B. Antibody mediated protein $\mathrm{G}$ capture-based binding assay also show strong binding signal from round 9 relative to the starting population $(n=1)$. C. Flow cytometry evaluation binding to HeLa cells transfected with both EBOV GP plasmid (purple), MARV GP plasmid and pcDNA3.1 plasmid (gray) $(n=1)$. 


\section{Conclusion.}

Despite the advantages of generating aptamers through a cell SELEX approach, there is an inherent risk of slow target-specific enrichment due to the high number of off-target molecules that are also present on the surfaces of the target cells. Many groups have used variations on the hybrid SELEX approach to improve target specify and facilitate the enrichment of target specific sequences. ${ }^{30,31}$. For example, Hicke et al. generated aptamers that bind to Tenascin C protein by performing the initial SELEX with cells as target and the final rounds with purified proteins as targets ${ }^{32}$.Hela cell surfaces display a complex and higher number of off-target molecules, compared to the surfaces of VSV virus that display a single virally encoded surface protein. Switching from Hela surfaces to virus surfaces was intended to enrich for sequences that have affinity for EBOV GP and deplete the ones that bind to off-target molecules

In this work, we applied a hybrid SELEX approach to generate ssDNA aptamers that bind to EBOV GP displayed in a native context. The first six rounds of selection utilized EBOV GP-expressing HeLa cells as targets. Subtractive steps were performed on HeLa cells that were transfected with pcDNA3.1, a non-GP expressing plasmid. Flow cytometry was used to assess the binding of the rounds 5 and 6 populations (selected to bind EBOV GP displayed on HeLa cells), relative to the starting populations. The evolved populations bind to the EBOV GP (target) and a related GP from MARV. Also, both populations bind to HeLa cells that were transfected with the empty vector. We then performed three additional rounds with beads-immobilized rVSV/EBOV_GP particles as the selection target. The evolved population shows a stronger dose-dependent 
radioactive signal in filter retention assay compared to the starting library. Similarly, the evolved population shows a higher signal on immobilized rVSV/EBOV_GP in the bead retention assay used to evaluate target binding. Flow cytometry assessment suggests that the evolved population binds to HeLa cells transfected with EBOV GP (target), MARV GP (non-target GP), or pcDNA3.1 plasmid (non-target). Future works will evaluate the binding specificity of the evolved population by assessing binding to non-target virus particles and other cells.

\section{Addendum}

The next steps for this work include the identification and characterization of the individual sequences from the evolved population. Identifying individual sequences would require a low-throughput screening approach, where the library would be cloned into plasmids and the plasmids sequenced through sanger sequences. Alternatively, the final population and other intermediate populations could be subjected to deep sequencing. In this approach, the enrichment trajectories of individual sequences or clusters of sequences could be analyzed in a high -throughput manner. The sequences obtained would be evaluated for their ability to bind to GP from EBOV. Data from this chapter showed pan-filovirus GP recognition; therefore, the sequences would be evaluated for their ability to bind to related GP such as GP from MARV and other EBOV species. Finally, the sequences that bind to filovirus GP may block virus infection. Our collaborators would perform a filovirus neutralization screen on the filovirus GP binding sequences to identify the ones that neutralize MARV, EBOV, or both. 


\section{Material and methods}

\section{Cell SELEX}

The selection utilized an ssDNA library with 40 nucleotides random region flanked by two fixed primer binding sites (5'-TAGGGAAGAGAAGGACATATGAT-40NTTGACTAGTACATGACCACTTGA-3') which was synthesized by Integrated DNA Technology (IDT, Coralville, IA). The 3' primer was annealed to the libraries before every round during the thermal renaturing step in every round. In round one, 1 nmole of ssDNA was annealed to the 3 'primer by heating the sequences at $90^{\circ} \mathrm{C}$ for 3 min and cooling gradually to room temperature (1.3-fold excess primer was used). The thermally renatured library is cooled on ice for $10 \mathrm{~min}$ before it is incubated with cells. For cell SELEX, we followed procedures as described by Sefah et al. $(2010)^{29}$. Briefly, HeLa cells were lifted off the $100 \mathrm{~mm}$ cell culture plates washed with $10 \mathrm{ml}$ cold PBS by spinning the cells at $450 \mathrm{rcf}$ for $5 \mathrm{~min}$. The supernatant was discarded, and the cell resuspended in cold PBS. For the first round, 10 nmoles of thermally renatured ssDNA were incubated with the cells in a selection buffer $(50 \mathrm{mM}$ Tris $\mathrm{HCl} \mathrm{pH}$ 7.5, $140 \mathrm{mM}$ $\mathrm{NaCl}, 5 \mathrm{mM} \mathrm{KCl}, 5 \mathrm{mM} \mathrm{CaCl}_{2}, 2 \mathrm{mM} \mathrm{MgCl} 2$ ) for 1 hour at $4^{\circ} \mathrm{C}$ in. Following the incubation, the cells were washed three times with $1 \mathrm{~mL}$ selection buffer. The cell pellet following the final wash was resuspended in water and heated at $90^{\circ} \mathrm{C}$ for $10 \mathrm{~min}$ and spun for 16, $000 \mathrm{rcf}$ for $5 \mathrm{~min}$. The supernatant was collected, and the recovered sequence used as a template for PCR amplification. PCR was performed using conditions described in chapter 4 . The subsequent rounds included $1 \mathrm{mg} / \mathrm{ml}$ of salmon sperm DNA during the incubation step to reduce non-specific binding of the library. Also, subtractive steps were performed against HeLa cells that were transfected with pcDNA3.1 plasmid. 


\section{Immobilizing rVSV/EBOV_GP on the surface of beads}

The rVSV/EBOV_GP particles were immobilized on beads surfaces as follows. $100 \mu \mathrm{L}$ (1.5 mg) of Dyna beads ${ }^{\text {TM }}$ Protein G (Thermo fisher Scientific, Waltham, MA) were transferred into a tube and washed with PBS. The washed beads were collected by placing the tube on a magnet. $60 \mu \mathrm{g}$ of monoclonal antibody KZ52 in PBS (pH 7.4) with $0.01 \%$ Tween was added to the beads and incubated at room temperature with rotation for $20 \mathrm{~min}$. The KZ52 capture beads were collected and washed twice with PBS. The capture efficiency was assessed by measuring the absorbance at $280 \mathrm{~nm}$ of the KZ52 solution before and after incubation with beads. Concentration of KZ52 sample before capture was $0.06 \mathrm{mg} / \mathrm{ml}$. There was about $90 \%$ drop in the concentration of the KZ52 samples $(0.015 \mathrm{mg} / \mathrm{ml})$ following incubation with the beads, suggesting capture of antibodies on the beads surface $10^{7} \mathrm{pfu}$ of purified rVSV/EBOV_GP particles were added to $50 \mu \mathrm{L}$ of KZ52 coated Dyna beads ${ }^{\mathrm{TM}}$ Protein G in PBS with $0.01 \%$ Tween and incubated at $37^{\circ} \mathrm{C}$ for 1 hour. The beads were collected and washed twice with selection buffer.

\section{Bead-based Selection on immobilized rVSV/EBOV_GP}

For the bead-based selection rounds, 100 pmoles thermally renatured ssDNA annealed to ODN was incubated with $50 \mu \mathrm{L}$ of immobilized rVSV/EBOV_GP particles in selection buffer at $25^{\circ} \mathrm{C}$ for $30 \mathrm{~min}$. The virus immobilized beads were washed 3 times with 500 $\mu \mathrm{L}$ of selection buffer and then heated at $95^{\circ} \mathrm{C}$ for $10 \mathrm{~min}$ in water to release any bound ssDNA. The eluted ssDNA was PCR amplified using condition described in chapter 4. 


\section{Beads retention binding assay.}

To generate radiolabeled sequences, forward primer was labeled at the $5^{\prime}$ end and used for the PCR amplification step. The primer was labeled with $\left[\gamma^{3}{ }^{32} \mathrm{P}\right]$-ATP (PerkinElmer, Waltham, MA ) by T4-polynucleotide kinase (NEB, Ipswich, MA), according to manufacturer's instruction. Following PCR, we performed strand separation. For the beads retention assays, $10 \mathrm{nM}$ (1pmole) radiolabeled ssDNA was annealed to $13 \mathrm{nM}$ of 3 ' primer and incubated with $25 \mu \mathrm{L}$ of immobilized rVSV/EBOV_GP particles in SELEX buffer (50 mM Tris $\mathrm{HCl} \mathrm{pH}$ 7.5, $140 \mathrm{mM} \mathrm{NaCl}, 5 \mathrm{mM} \mathrm{KCl}, 5 \mathrm{mM} \mathrm{CaCl} 2,2 \mathrm{mM} \mathrm{MgCl}_{2}$ ) and allowed to equilibrate for $30 \mathrm{~min}$ at $37^{\circ} \mathrm{C}$. The samples were washed 3 times with $200 \mu \mathrm{L}$ of selection buffer. The beads transferred into liquid scintillation vials with $3 \mathrm{ml}$ scintillation fluid. Radioactivity retained on the beads was quantified using a scintillation counter. Percent ssDNA bound was calculated by determining the fraction of radioactivity on the beads relative to the input radioactivity. 


\section{Reference}

(1) Lee, J. E.; Saphire, E. O. Ebolavirus Glycoprotein Structure and Mechanism of Entry. Future Virology. NIH Public Access 2009, pp 621-635. https://doi.org/10.2217/fvl.09.56.

(2) Shurtleff, A. C.; Whitehouse, C. A.; Ward, M. D.; Cazares, L. H.; Bavari, S. PreSymptomatic Diagnosis and Treatment of Filovirus Diseases. Frontiers in Microbiology. Frontiers Media S.A. 2015. https://doi.org/10.3389/fmicb.2015.00108.

(3) Sheridan, C. Merck Vaccine Heads Ebola Countermeasures. Nat. Biotechnol. 2018, 36 (7), 563-565. https://doi.org/10.1038/nbt0718-563a.

(4) Geisbert, T. W. First Ebola Virus Vaccine to Protect Human Beings? The Lancet. Lancet Publishing Group February 4, 2017, pp 479-480. https://doi.org/10.1016/S0140-6736(16)32618-6.

(5) Lucey, D. R. New Treatments for Ebola Virus Disease. The BMJ. BMJ Publishing Group September 6, 2019. https://doi.org/10.1136/bmj.15371.

(6) Mulangu, S.; Dodd, L. E.; Davey, R. T.; Tshiani Mbaya, O.; Proschan, M.; Mukadi, D.; Lusakibanza Manzo, M.; Nzolo, D.; Tshomba Oloma, A.; Ibanda, A.; Ali, R.; Coulibaly, S.; Levine, A. C.; Grais, R.; Diaz, J.; Lane, H. C.; MuyembeTamfum, J.-J.; the PALM Writing Group. A Randomized, Controlled Trial of Ebola Virus Disease Therapeutics. N. Engl. J. Med. 2019, 381 (24), 2293-2303. https://doi.org/10.1056/NEJMoa1910993.

(7) Flyak, A. I.; Ilinykh, P. A.; Murin, C. D.; Garron, T.; Shen, X.; Fusco, M. L.; Hashiguchi, T.; Bornholdt, Z. A.; Slaughter, J. C.; Sapparapu, G.; Klages, C.; 
Ksiazek, T. G.; Ward, A. B.; Saphire, E. O.; Bukreyev, A.; Crowe, J. E.

Mechanism of Human Antibody-Mediated Neutralization of Marburg Virus. Cell

2015, 160 (5), 893-903. https://doi.org/10.1016/j.cell.2015.01.031.

(8) Shimojima, M.; Takada, A.; Ebihara, H.; Neumann, G.; Fujioka, K.; Irimura, T.;

Jones, S.; Feldmann, H.; Kawaoka, Y. Tyro3 Family-Mediated Cell Entry of Ebola and Marburg Viruses. J. Virol. 2006, 80 (20), 10109-10116.

https://doi.org/10.1128/JVI.01157-06.

(9) Simmons, G.; Reeves, J. D.; Grogan, C. C.; Vandenberghe, L. H.; Baribaud, F.; Whitbeck, J. C.; Burke, E.; Buchmeier, M. J.; Soilleux, E. J.; Riley, J. L.; Doms, R. W.; Bates, P.; Pöhlmann, S. DC-SIGN and DC-SIGNR Bind Ebola Glycoproteins and Enhance Infection of Macrophages and Endothelial Cells. Virology 2003, 305 (1), 115-123. https://doi.org/10.1006/viro.2002.1730.

(10) Alvarez, C. P.; Lasala, F.; Carrillo, J.; Muñiz, O.; Corbí, A. L.; Delgado, R. CType Lectins DC-SIGN and L-SIGN Mediate Cellular Entry by Ebola Virus in Cis and in Trans . J. Virol. 2002, $76(13), 6841-6844$.

https://doi.org/10.1128/jvi.76.13.6841-6844.2002.

(11) Gramberg, T.; Soilleux, E.; Fisch, T.; Lalor, P. F.; Hofmann, H.; Wheeldon, S.; Cotterill, A.; Wegele, A.; Winkler, T.; Adams, D. H.; Pöhlmann, S. Interactions of LSECtin and DC-SIGN/DC-SIGNR with Viral Ligands: Differential PH Dependence, Internalization and Virion Binding. Virology 2008, 373 (1), 189-201. https://doi.org/10.1016/j.virol.2007.11.001.

(12) Nanbo, A.; Imai, M.; Watanabe, S.; Noda, T.; Takahashi, K.; Neumann, G.; Halfmann, P.; Kawaoka, Y. Ebolavirus Is Internalized into Host Cells via 
Macropinocytosis in a Viral Glycoprotein-Dependent Manner. PLoS Pathog. 2010, 6 (9). https://doi.org/10.1371/journal.ppat.1001121.

(13) Chandran, K. Endosomal Proteolysis of the Ebola Virus Glycoprotein Is Necessary for Infection. Science (80-. ). 2005, 308 (5728), 1643-1645. https://doi.org/10.1126/science.1110656.

(14) Qiu, X.; Wong, G.; Audet, J.; Bello, A.; Fernando, L.; Alimonti, J. B.; FaustherBovendo, H.; Wei, H.; Aviles, J.; Hiatt, E.; Johnson, A.; Morton, J.; Swope, K.; Bohorov, O.; Bohorova, N.; Goodman, C.; Kim, D.; Pauly, M. H.; Velasco, J.; Pettitt, J.; Olinger, G. G.; Whaley, K.; Xu, B.; Strong, J. E.; Zeitlin, L.; Kobinger, G. P. Reversion of Advanced Ebola Virus Disease in Nonhuman Primates with ZMapp. Nature 2014, 514 (7520), 47-53. https://doi.org/10.1038/nature13777.

(15) Mulherkar, N.; Raaben, M.; de la Torre, J. C.; Whelan, S. P.; Chandran, K. The Ebola Virus Glycoprotein Mediates Entry via a Non-Classical DynaminDependent Macropinocytic Pathway. Virology 2011, 419 (2), 72-83. https://doi.org/10.1016/j.virol.2011.08.009.

(16) Aleksandrowicz, P.; Marzi, A.; Biedenkopf, N.; Beimforde, N.; Becker, S.; Hoenen, T.; Feldmann, H.; Schnittler, H.-J. Ebola Virus Enters Host Cells by Macropinocytosis and Clathrin-Mediated Endocytosis. https://doi.org/10.1093/infdis/jir326.

(17) Tuerk, C.; Gold, L. Systematic Evolution of Ligands by Exponential Enrichment: RNA Ligands to Bacteriophage T4 DNA Polymerase. Science (80-. ). 1990, 249 (4968), 505-510. https://doi.org/10.1126/science.2200121.

(18) Ellington, A. D.; Szostak, J. W. In Vitro Selection of RNA Molecules That Bind 
Specific Ligands. Nature 1990, 346 (6287).

(19) Tawiah, K. D.; Porciani, D.; Burke, D. H. Toward the Selection of Cell Targeting Aptamers with Extended Biological Functionalities to Facilitate Endosomal Escape of Cargoes. Biomedicines 2017, 5 (3). https://doi.org/10.3390/biomedicines5030051.

(20) Zhou, J.; Rossi, J. Aptamers as Targeted Therapeutics: Current Potential and Challenges. Nature Reviews Drug Discovery. Nature Publishing Group March 1, 2017, pp 181-202. https://doi.org/10.1038/nrd.2016.199.

(21) Wang, J.; Jiang, H.; Liu, F. In Vitro Selection of Novel RNA Ligands That Bind Human Cytomegalovirus and Block Viral Infection; 2000.

(22) Kwon, H.-M.; Lee, K. H.; Han, B. W.; Han, M. R.; Kim, D. H.; Kim, D.-E. An RNA Aptamer That Specifically Binds to the Glycosylated Hemagglutinin of Avian Influenza Virus and Suppresses Viral Infection in Cells. PLoS One 2014, 9 (5), e97574. https://doi.org/10.1371/journal.pone.0097574.

(23) B Gopinath, S. C.; Misono, T. S.; Kawasaki, K.; Mizuno, T.; Imai, M.; Odagiri, T.; R Kumar, P. K.; Penmetcha R Kumar, C. K. An RNA Aptamer That Distinguishes between Closely Related Human Influenza Viruses and Inhibits HaemagglutininMediated Membrane Fusion. https://doi.org/10.1099/vir.0.81508-0.

(24) Liang, H. R.; Hu, G. Q.; Li, L.; Gao, Y. W.; Yang, S. T.; Xia, X. Z. Aptamers Targeting Rabies Virus-Infected Cells Inhibit Street Rabies Virus in Vivo. Int. Immunopharmacol. 2014, 21 (2), 432-438.

https://doi.org/10.1016/j.intimp.2014.03.020.

(25) Cohen, C.; Forzan, M.; Sproat, B.; Pantophlet, R.; McGowan, I.; Burton, D.; 
James, W. An Aptamer That Neutralizes R5 Strains of HIV-1 Binds to Core Residues of Gp120 in the CCR5 Binding Site. Virology 2008, 381 (1), 46-54. https://doi.org/10.1016/j.virol.2008.08.025.

(26) Sayer, N.; Ibrahim, J.; Turner, K.; Tahiri-Alaoui, A.; James, W. Structural Characterization of a 2'F-RNA Aptamer That Binds a HIV-1 SU Glycoprotein, Gp120. Biochem. Biophys. Res. Commun. 2002, 293 (3), 924-931. https://doi.org/10.1016/S0006-291X(02)00308-X.

(27) Mufhandu, H. T.; Gray, E. S.; Madiga, M. C.; Tumba, N.; Alexandre, K. B.; Khoza, T.; Wibmer, C. K.; Moore, P. L.; Morris, L.; Khati, M. UCLA1, a Synthetic Derivative of a Gp120 RNA Aptamer, Inhibits Entry of Human Immunodeficiency Virus Type 1 Subtype C. J. Virol. 2012, 86 (9), 4989-4999. https://doi.org/10.1128/jvi.06893-11.

(28) Gopinath, S. C. B.; Hayashi, K.; Kumar, P. K. R. Aptamer That Binds to the GD Protein of Herpes Simplex Virus 1 and Efficiently Inhibits Viral Entry. J. Virol. 2012, 86 (12), 6732-6744. https://doi.org/10.1128/jvi.00377-12.

(29) Sefah, K.; Shangguan, D.; Xiong, X.; O’Donoghue, M. B.; Tan, W. Development of DNA Aptamers Using Cell-Selex. Nat. Protoc. 2010, 5 (6), 1169-1185. https://doi.org/10.1038/nprot.2010.66.

(30) Mallikaratchy, P. Evolution of Complex Target SELEX to Identify Aptamers against Mammalian Cell-Surface Antigens. Molecules. MDPI AG February 1, 2017, p 215. https://doi.org/10.3390/molecules22020215.

(31) Burke, D. H.; Scates, L.; Andrews, K.; Gold, L. Bent Pseudoknots and Novel RNA Inhibitors of Type 1 Human Immunodeficiency Virus (HIV-1) Reverse 
Transcriptase. J. Mol. Biol. 1996, 264 (4), 650-666.

https://doi.org/10.1006/jmbi.1996.0667.

(32) Hicke, B. J.; Marion, C.; Chang, Y. F.; Gould, T.; Lynott, C. K.; Parma, D.;

Schmidt, P. G.; Warren, S. Tenascin-C Aptamers Are Generated Using Tumor Cells and Purified Protein. J. Biol. Chem. 2001, 276 (52), 48644-48654.

https://doi.org/10.1074/jbc.M104651200. 


\title{
Chapter 6: Perspective on Aptamers targeting virus
}

\section{surfaces: rapid development of diagnostic probes.}

This chapter will be modified for submission as review manuscript, Anticipated Authors Kwaku D. Tawiah, David Porciani, and Donald H. Burke."

\begin{abstract}
Rapid, accurate, and simple diagnostics and subtyping of viral diseases are crucial for the overall disease management and control of outbreaks and pandemics. The lack of pointof-care diagnostics assays can exacerbate the health and economic impacts caused by poor control management of virus outbreaks. The rise in the frequency of viral disease outbreaks from emerging and novel viruses highlights the need for alternative diagnostic probes. The development of such probes should be able to be done quickly to support the management of novel and emerging viruses when outbreaks occur. Here, we highlight nucleic acid aptamers as diagnostic probes that could be developed rapidly. Aptamers can be developed to have affinity for virus surfaces through a process called SELEX.

Advances in the SELEX process, such as the use of modified libraries, automation and adaptation of microfluidics, and bioinformatics-guided screening, make aptamers an option for the rapid development of affinity probes for virus surfaces.
\end{abstract}

\section{Introduction.}

Viruses can cause life-threatening diseases in humans. Viruses that annually circulate among humans pose a significant challenge to healthcare systems globally and impacts the global economy ${ }^{1}$. There have been a number of virus disease outbreaks in recent years, including Ebola virus disease (EVD), Zika fever, severe acute respiratory 
syndrome (SARS), and the ongoing coronavirus 2019 disease (COVID-19) pandemic. Diagnostics impact more than half of clinical decision making for virus outbreaks, including treatment approaches, public health surveillance and control ${ }^{2-5}$. Early and reliable detection of infectious pathogens is vital for successful management and treatment. Moreover, unnecessary diagnostic tests and therapies, including hospitalizations, can be avoided or reduced. Finally, effective diagnostic tests significantly help with the allocation of limited resources, especially during pandemics ${ }^{6-8}$. For example, essential health care stockpiles, such as antivirals and protective equipment can be properly distributed.

Over the years, traditional diagnostic methods based on viral cultures, plaque assays, and electron microscopy have been replaced by innovative and convenient molecular biology techniques, such as PCR-based assays, immunoassays (ELISA), and molecular probes $(\text { FISH })^{9,10}$. These molecular biology assays are usually faster, sensitive, and robust. For example, the two most common variants of PCR based assays, real-time quantitative PCR (qPCR), and reverse transcription PCR (RT-PCR) detect viral genomes. qPCR quantifies virus DNA in real-time, while RT-PCR has an additional RT step that converts RNA to cDNA (complementary DNA) before the amplification step. Both assays have become benchmarks for testing respiratory and other viral infections because they combine both qualitative detection with quantitative analysis of pathogens ${ }^{11}$. Immunoassays have also been used extensively in clinical and research laboratories as diagnostic probes for viral infections. Antibodies are good reagents for virus detection and hence, suitable for qualitative screening for infection as opposed to virus quantification. 
Both PCR-based assays and immunoassays have proven effective through outbreaks and pandemics, especially when used in combination ${ }^{12}$ to provide more comprehensive and impartial diagnostic results. Unfortunately, both approaches display some limitations. Oligonucleotide primers used in PCR-based assays are designed based on the viral genome sequences. However, some viral genomes are subjected to high mutation rates, thus requiring constant updates and quality control of primer design. Also, PCR-based assays require sophisticated thermocyclers that present logistic challenges in resource limiting settings, and they can be expensive to operate. Although immunoassays have proven to be fast useful, and they display a low complexity, a major problem is the batchto-batch variation in the antibody production that can affect the reliability of diagnostic results. In fact, the integrity of immunoassays, such as ELISA, depends on the choice of monoclonal antibodies. However, it is very challenging to maintain antibody producing cell lines over an extended period. Similar to some reagents (such as enzymes) used in PCR based assays, antibodies need a cold chain to maintain their shelf life (12-24 months at $2-8^{\circ} \mathrm{C}$ ). Unfortunately, this might limit the use of antibodies and serological assays during viral disease outbreaks in rural areas. Finally, the development of immunoassays could take some time (i.e., 3-6 months for mAb), especially during the outbreak of novel viruses such as the current COVID-19 pandemic $^{13}$. In consideration of these pitfalls, a great amount of research and resources are focused on developing complementary approaches that could address some of the aforementioned limitations associated with the current viral diagnostic assays. Several reviews have suggested potential solutions to be taken into account for developing the next generation of diagnostic probes. However, 
many of the suggested novel-approaches still rely either on existing probes, such as antibodies, or modifications of PCR based assays to make them faster.

Among the emerging affinity probes that could be used in the development of new biosensors, aptamers display several advantages and properties that could address some of the existing pitfalls associated with antibodies. Aptamers are single-chain oligonucleotides that fold into unique 3D structures that bind their molecular targets with high affinity and specificity. They can fold into a variety of 3D structures, such as stemloops, bulges, pseudoknots, kinks, and G-quadruplexes, providing a diverse range of recognition surfaces. Aptamers are developed through a combinatorial chemistry process called SELEX (Systematic Evolution of Ligands by EXponential enrichment) ${ }^{14-16}$. The SELEX process identifies ligands from a highly diverse library of nucleic acids (typically $10^{11}-10^{16}$ ) through affinity capture and amplification. Over the years, high-affinity aptamers have been developed to bind to a variety of targets including small molecules ${ }^{17}$, $\operatorname{proteins}^{18,19}$, viruses ${ }^{20,21}$, bacteria ${ }^{22}$, and other nucleic acids (such as pri-miRNAs) ${ }^{23}$. The wide range of molecules that can be targeted by aptamers offers a huge advantage compared to other diagnostic probes. Additionally, compared to other affinity probes such as antibodies (Abs) and antibody fragments (Fabs), aptamers offer high batch-tobatch integrity, ease of chemical conjugation to functional groups of other probes (such as biomolecules, small organic dyes, etc.), long term stability, and long shelf life due to their chemical nature and synthesis process. Also, aptamers are smaller in size $(\sim 3 \mathrm{~nm}, 6-$ $30 \mathrm{kDa}$ ) allowing them to access surfaces on their target molecules that can be inaccessible to larger ligands, such as Abs $(10-15 \mathrm{~nm}, \sim 150 \mathrm{kDa})^{24}$. Overall, aptamers are 
very versatile and can be easily integrated into existing viral diagnostic platforms. Thus, they can fill some of the current gaps and push the frontiers for the rapid development of improved viral diagnostics.

Aptamers and the SELEX technology emerged as promising tools in the biotechnology industry in the early 1990s. Despite their immense potential as versatile affinity probes, aptamers are yet to see commercial success. Many reviews have addressed some of the reasons for this slow progress ${ }^{25,26}$. When they emerged, aptamers were perceived as direct competition/replacement for Abs, which are well-established with huge amounts of resource investments. Thus, it did not make sense economically to sidestep such investments and invest in the development of novel technologies that offer promise. Moreover, Abs have provided some satisfactory results both in virus disease therapy (e.g., Palivizumab for respiratory syncytial virus RSV \& REGNEB3 for Ebola) and diagnostics ${ }^{27,28}$. In addition, patent protections by the initial inventors contributed to slowing down the commercial launch of aptamers ${ }^{19}$. These, among other reasons have resulted in the majority of aptamer research and development being done at low-scale academic research labs. Despite these limitations, a handful of companies are now developing aptamer diagnostics and therapeutics for the commercial market. Companies like SomaLogic, Antigen LLC, Vivonics, and Aptamer Group are at the forefront of these advancements. Focusing on niche sectors such as the rapid development of viral diagnostics might present a better approach to ramping up commercial aptamer development in a way that complements well-established diagnostics approaches. Viral surfaces present a target-rich interface for aptamers. Aptamers targeting viruses provide 
unique reagents for probing virus surfaces and dissecting viral-host interactions. This review examines virus-targeting aptamers as an option for developing diagnostic probes that can complement resources. We highlight some of limitations in developing aptamers that have affinity for virus surfaces. Finally, we outline recent advancements that have been shown to address some of the challenges highlighted. The advances discussed in this review focuses on three areas: i) nucleic acid chemistry and diversity, ii) selection efficiency, and iii) low and high-throughput sequencing and bioinformatics guided post selection screening.

\section{In vitro development of virus-targeting aptamers.}

SELEX is an in vitro evolution process that identifies aptamers from a library of

oligonucleotide sequences. To develop virus-targeting aptamers, nucleic acid pools with a high sequence variability (e.g., $10^{11}-10^{16}$ different molecules) are incubated with the virus target. After binding the target virus protein, the bound aptamers are separated from the unbound sequences in a partitioning step. In these selections, the molecular target is represented by a virus surface protein, which is displayed in different forms: as a recombinant virus protein, on the surface of viruses or virus surrogates (e.g., chimeric and pseudotyped viruses). Research work done on highly virulent viruses such as filoviruses, require specialized containment laboratories (Biosafety level 4, BSL4). BSL4 laboratories have the highest level of biocontainment precautions. There are currently only 5 operational BSL4 facilities within the United states. BSL1 and BSL2 surrogates of BSL4 provide excellent alternatives to studying these viruses without BSL4 facilities. 
The partition step is the key for any selection, as it is the step-in which aptamers with affinity for the target are separated from non-binding or weakly-binding species. The aptamer field has developed several partition platforms, including the use of functionalized magnetic beads, affinity resins, and nitrocellulose membranes. Differential electrophoretic properties of bound and unbound species in gels has also been exploited as a means of separation. The choice of partitioning approach in a selection contributes to the level of off-target, which can either facilitate the enrichment of target specific sequences or off-target. To ensure a successful and rapid selection of aptamers, an ideal partition platform should maintain a low, non-specific background binding $(<1 \%$ of the fraction bound) to exponentially enrich the candidate aptamer sequences ${ }^{29,30}$.

Aptamer specificity can also be improved by performing subtractive steps to deplete sequences that have affinity for non-target proteins. For example, influenza A hemagglutinin can be used as a non-target for a selection to generate aptamers that bind to influenza B hemagglutinin. In the subtractive step, the library is incubated with nontarget and the unbound sequences are recovered and subsequently incubated with the target. Aptamer selections are often designed to achieve highly specific recognition, but SELEX can also generate aptamers with broad-spectrum recognition of a distinct virus species. For instance, our lab generated aptamers that recognize multiple strains of the Reverse Transcriptase of HIV-1 ${ }^{31}$. Broad-spectrum aptamers would likely bind to conserved regions on the target viruses and could potentially be utilized as off the-shelf diagnostics for a strains or species of viruses. 
At the end of a selection, specific sequences are identified through sequencing. Aptamers that possess desired binding properties (e.g., high specificity and affinity) and/or distinct functional activity (e.g., virus neutralization) are chemically synthesized and characterized. Functional structures of an aptamers do not necessarily utilize the full sequence that come out of the selection. For example, some functional structures of aptamers have been shown to be independent of the primer binding sites. In addition, not all the nucleotides in the random region are engaged in the formation of the functional structure. The minimal sequence required for binding is determined through structural predictions and series sequence truncations. Shorter sequences are preferred over shorter ones because they are cheaper and easier to chemically synthesize. Moreover, aptamer manipulations such as the generation of multimers, chemical conjugation to other molecules are more efficient with shorter sequences.

Developing virus-targeting aptamers using recombinant purified proteins as targets (protein-SELEX) is a common approach in the field ${ }^{32}$. Purified proteins tend to be free from non-target protein contaminants, thus limiting off-target binding and increasing the efficiency of SELEX. Recombinant protein samples can display homogenous epitopes and structures. In contrast, when the same target virus proteins are expressed and displayed on the surfaces of virus surrogates or mammalian cells. Those membranes may contain many additional non-target receptors that can be targeted by aptamers. As a consequence, increased levels of off-target binding hamper the effective enrichment of aptamer sequences that bind the target of interest $\mathrm{t}^{30,33}$. Nevertheless, there are several 
disadvantages associated with the use of purified recombinant proteins as SELEX targets. First, it can be challenging to purify certain surface virus proteins, such as glycoproteins. To facilitate the protein purification process, several modifications can be introduced, e.g., amino acid mutations, truncations of domains, and addition of foreign domains as tags. In addition, proteins expressed and purified from bacteria lack the post-translation modification such as glycosylation, which is present in eukaryotic systems. Some of these modifications could impact the structure, thus causing structural differences between native form of the protein and the purified variant. Aptamers with affinity for purified proteins may not bind to the native displayed form. For example, aptamers selected to bind to recombinant human epidermal growth factor receptor (hEGFRvIII) produced in E-coli, failed to bind to the same protein expressed from eukaryotic cells due to the presence of glycans on the surface of the eukaryotic cell displayed hEGFRvIII ${ }^{34}$. These pitfalls hamper the effectiveness of a platform designed for the rapid development of viral diagnostic probes. Targets and conditions used in the development of diagnostic probes including aptamer-based probes should be as close to the natural target as possible if not the same.

The use native displayed proteins as selection target can address some of the pitfalls associated with the use purified recombinant forms of the protein target. "Virus SELEX", consist of a collection of SELEX strategies that utilize intact virus particles as selection targets. To select for aptamers that have affinity for native virus proteins, three main types of Virus SELEX can be designed: i) SELEX with whole viruses as targets, ii) SELEX with virus surrogates as targets, and iii) SELEX with eukaryotic cell-displayed virus proteins as targets. Targeting virus proteins in their native environment can yield 
reliable aptamers with enhanced potential to be used as diagnostics (and/or potent neutralizing activity).

There are a number of reports that have utilized the virus SELEX approach to develop aptamer that have affinity for virus surfaces. Percze et al. performed a virus SELEX that utilized a chemically inactivated respiratory syncytial virus (RSV) particles as targets. They identified a set of ssDNA aptamers that have affinity for RSV surfaces. Filter retention assays show that the aptamers are capable of detecting clinically relevant concentrations of RSV $\left(10^{5} \mathrm{pfu}\right)$ in throat swabs. RSV causes respiratory infections in humans and is the most common cause of lower respiratory tract infections in children. RSV infection can cause severe complications such as Pneumonia and bronchiolitis in children and immunocompromised patients when they are not detected early. Therefore, aptamers that can detect RSV can serve as excellent tools for developing RSV biosensors.

Similar approach was used to generate RNA aptamers that differentially binds to the surface of closely related influenza A strains. Influenza virus displays two glycoproteins, hemagglutinin (HA) and neuraminidase (NA) on their surface. The similarities between subunits of influenza virus displayed HA ranges between 80 and $90 \%$ at the amino acid level. Most monoclonal antibodies that have affinity for HA are unable to distinguish between influenza subtypes. An RNA aptamer (P30-10-16) selected to have affinity for strain A/Panama/2007/1999(H3N2) binds and blocks infection of that $\operatorname{strain}^{35}$. The same aptamer fails to bind to HA displayed on other human influenza viruses including closely related HA from stains within the same subtype H3N2. 
Diagnostic assays that rely on sandwich strategies benefit from the ability of virus SELEX to generate aptamer pairs that target different epitopes on virus surfaces. Monoclonal antibodies are typically generated towards the most immunogenic epitopes. Therefore, it can be challenging to identify antibody pairs that can be utilized reliably in a sandwich assay. Many antibody based sandwich assays utilize polyclonal antibodies, which can result in false positives ${ }^{36,37}$. The use polyclonal antibodies could result in offtarget binding to contaminants in specimens. Park J et al. reported a virus SELEX that generated a set of ssDNA aptamers that bind to the surface of bovine viral diarrhea virus (BVDV). Assays performed to evaluate binding competition between the aptamers identified aptamer pairs that bind to different epitopes on the virus surfaces. When utilized in sandwich assays, these aptamer pairs captured and detected BVDV with clinically relevant limit of detection $\left(500 \mathrm{TCID}_{50} / \mathrm{ml}\right)$.

\section{Strategies to improve the SELEX efficiency and affinity of virus-targeting aptamers}

To use SELEX as an efficient and rapid reagent platform, both its success rate and speed of aptamer production need to improve significantly. Conventional SELEX has a low success rate $(\sim 30 \%)$. SELEX failure can be exacerbated when a protein target has low isoelectric point values ( $\mathrm{pI}<7$ at physiological condition) possibly due to a net negative charge on the target. Nucleic acid aptamers are negatively charged due to their phosphate backbone, thus high electrostatic repulsion between nucleic acids and targets could prevent binding ${ }^{38,39}$. For aptamers to better options for developing affinity reagents for virus detection, the success rate and development time for in vitro selections would have to improve (success rate $>90 \%$ ). However, many factors such as library design and 
make, SELEX platform used, and the chemical properties of the target can influence the success of a virus SELEX. Naturally occurring nucleic acids lack a high chemical diversity because sequences are limited to the four natural nucleotides. They are also susceptible to degradation by serum nucleases, which can limit their utility in assays involving bodily fluids. Also, traditional selections are limited by low efficiency partitioning systems that result in long and cumbersome rounds of selection. Low efficiency partitioning system such as nitrocellulose filters tend to have high non-specific binding or unable to eliminate sequences that bind to non-targets. This results in a laborintensive process of screening and characterization of the candidate aptamer sequences that is performed to ultimately identify winning aptamers. A summary of the steps and the average time used to successfully generate a small set (2-5) of fully-characterized aptamers is summarized in Fig 6.1. Over the years, there have been several approaches to improve nucleic acid chemical diversity and utility. Also, the adoption of microfluidics and high-throughput sequencing in aptamer development has improved selection effeciency. 


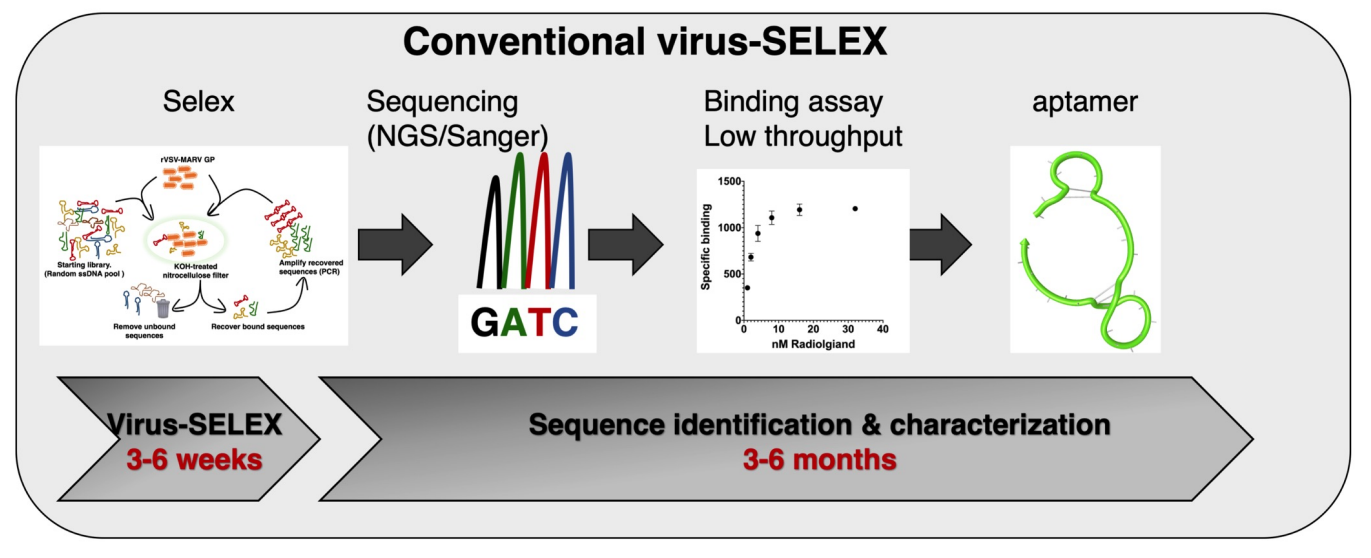

Figure 6.1. Overview of the conventional aptamer selection process. Conventional SELEX utilize inefficient partitioning platforms. It takes about 10-15 rounds to generate enriched sequences to the target. Sequencing, screening and aptamer are all done in a low-throughput manner and can be time consuming. 


\section{Aptamers with enhanced chemical diversity improve SELEX success rate}

Many modifications have been employed to improve the function and versatility of aptamers as mainstream affinity probes. These modifications are often classified based on their outcome on aptamer function and utility: 1) modifications that enhance the half-life of aptamer in biological fluids against enzymatic degradation and 2) modifications that expand the set of possible chemical interactions between aptamers and their target proteins. Modification done on either the phosphate backbone or sugar moiety improves the resistance of nucleic acids against nucleases in biological fluids. Biological fluids such as blood, urine and sputum are full of nucleases. Therefore, it is important that aptamers display a certain degree of resistance to the nuclease-mediated enzymatic degradation that can occur in biological fluids. Naturally occurring nucleic acids have 2'deoxy (DNA) and 2'hydroxyl (RNA) on the sugar ring which makes them susceptible to nucleases. Particularly, the 2'hydroxyl group in RNA can easily deprotonate and facilitate a nucleophilic attack on the neighboring phosphorus in the phosphodiester bond to create a cyclic phosphodiester which gets hydrolyzed. Due to this, natural nucleic acids have a short half-life in serum $(<1$ minute for RNA, $\sim 1-2$ hours for DNA). One of the most common and utilized modifications is the substitution of the 2' hydroxyl with other chemical groups such as 2'-fluoro, 2'amino, and 2'O-methyl. Several reports have shown that modifications at the 2' position of the ribose can extend the serum half-life of RNA oligonucleotides (e.g., aptamers and siRNAs) up to a few hours (1-5 hours). Modifications on the phosphodiester linkage and phosphate backbone have also been reported. Examples of such modifications include phosphorothioate (PT) linkage and triazole-mediated click linkages. PT linkages are created when the non-bridging oxygen 
in a phosphodiester bond is replaced with a sulfur. PT linkages share similar physical and chemical properties with phosphodiester linkages, but they show an enhanced serum stability. For instance, virus-inhibition screening identified a PT linkage-modified Gquadruplex oligonucleotide that binds to HIV gp120 with strong affinity and prevents cell entry of a pseudotyped $\mathrm{HIV}^{40}$. The PT linkage-modified oligo displayed serum stability and structural probing showed that the modification was responsible for the binding properties $^{41}$. There are several comprehensive reviews on these and other modifications ${ }^{42-44}$. Several reports have demonstrated that the success rate of aptamer selections can be improved by increasing the chemical diversity of the starting libraries, by using for instance nucleobase-modified aptamers ${ }^{39,45-47}$. Traditional aptamers are limited to a small set of physicochemical interactions with their molecular targets, such as hydrogen bonding, electrostatic interactions, and pi-pi stacking. Incorporating hydrophobic and aromatic structures (amino acid-like side chains) into the nucleic acid expands the available interaction repertoires and improves aptamer accessibility to epitopes that are not normally targeted by traditional aptamers. Hydrophobic and aromatic side chains are often directly involved in protein-protein interactions, including antibody-antigen recognition. However, generating the modified nucleotide monomers can be expensive, and some of the modified bases are not compatible with existing polymerases during the enzyme-mediated amplification steps in SELEX. Currently, the protein engineering and synthetic biology fields are utilizing directed evolution and rational design to generate polymerases that can incorporate non-canonical nucleotides ${ }^{48}$. Alternative approaches have been introduced that allow the use of more standard polymerases in combination 
with chemical workup of the synthesized polymer, such as the click-SELEX developed by the Mayer group ${ }^{47}$. Click SELEX incorporates modifications in each round of SELEX after the amplification step by using an alkyne modified library that substitutes regular thymidine with C5-ethynyl-2'-deoxyuridine (EdU).

Several nucleobase modifications have been recently developed. For example, Hirao's group developed an unnatural hydrophobic base pair between 7-(2-thienyl)-imidazo[4,5b] pyridine (Ds) and pyrrole-2- carbaldehyde $(\mathrm{Pa})^{49}$. The Benner group also developed 8 novel bases termed Artificially Expanded Genetic Information System (AEGIS) ${ }^{48}$. AEGIS exploits new hydrogen bonds by rearranging donor and acceptor atoms on natural bases. Sefah et al. successfully generated a high-affinity aptamer that targets a breast cancer cell line (MDA-MB-231) using the AEGIS library ${ }^{50}$. Two previous selection attempts on the MDA-MB-231 cell line using natural sequences had failed, thus suggesting chemical modification may contribute to binding. However, factors other than the chemical nature of the library may have contributed to the failed selections as well. Arguably, the most successful use of aptamers as diagnostic probes is the SomaScan ${ }^{\circledR}$ proteomic platform from SomaLogic. This platform uses a unique class of aptamers, the Slow Off-rate Modified Aptamers (SOMAmers) to bind and quantify specific proteins present in plasma in a multiplexed fashion. SOMAmers have amino-acid-like side chains at the 5-position of pyrimidine rings and/or the 7 and 8-positions on purines. In addition, SOMAmers are selected using a kinetic trap that favors aptamers with a slow dissociation rate from their targets $\left(\mathrm{t}_{1 / 2}>30 \text { mins- } 20 \mathrm{hr}\right)^{45,51-54}$. 


\section{Improving selection efficiency through microfluidic-based SELEX}

Microfluidic-based SELEX and capillary electrophoresis-based SELEX offers an attractive and approach to improving the efficiency, multiplexing, and speed of in vitro aptamer selections. Low efficiency partitioning methods tend to have higher background binding and are unable to adequately isolate target binders from non-target ones. Replacing low-efficiency partition methods (e.g., nitrocellulose filter-based selection) with more robust microfluidic-based methods can drastically improve the efficiency and throughput of aptamer discovery. A fully integrated microfluidic-based SELEX allows for automation of most steps in the aptamer selection process ${ }^{55}$ Microfluidics, sometimes referred to as lab-on-chip, can be considered a miniaturized version of a conventional laboratory. Microfluidic technology manipulates fluids (mix, separate, etc.) either passively through capillary action or actively using a combination of pumps and capillary action. This technology provides precise control over low amounts of protein targets in a reproducible fashion. A combination of this precision and a highly controlled microfluidics environment results in high stringency conditions applied during the selection rounds ${ }^{56,57}$. Although microfluidics technology has proven powerful, unfortunately, it is not accessible to many research labs because it requires specific expertise and can be expensive to operate. However, commercial diagnostic probe development platforms are better suited to benefit from this technology. Reported approaches of microfluidic-based and other technology based-SELEX fall under three main categories: (1) beads-based target immobilization ${ }^{57,58},(2)$ sol-gelbased $^{59,60}$, and (3) target immobilization-free (capillary electrophoresis) ${ }^{61-63}$. However, capillary electrophoresis SELEX (CE-SELEX) and magnetic bead-based SELEX 
associated with microfluidic technology (often called M-SELEX) are two of the most feasible platforms to select aptamers that target viruses. CE-SELEX was one of the first high-technologies based-SELEX techniques demonstrated. The approach separates bound sequences from unbound based on electrophoretic mobility change of target bound sequences. The efficacy of CE-SELEX results from a very efficient separation that is done completely in a solution without target immobilization. Using this technology, the Bowser group developed aptamers that have affinity for several proteins, such as human immunoglobulin E (IgE $)^{64}$, HIV reverse transcriptase ${ }^{63}$,Alpha-fetoprotein ${ }^{65}$, and human vascular endothelial growth factor $165(\text { hVEGF165 })^{66}$. Virus particles that are generated in the form of whole viruses or viral surrogates possess electrophoretic properties in solution, therefore their migration in solution can be manipulated with electric charge. ${ }^{67}$. Thus, CE-SELEX could represent an ideal approach to effectively isolate aptamers that bind virus particles, though, to the best our knowledge, it has not been demonstrated yet. CE-SELEX could be ideal for the rapid development of diagnostic probes because it does not require virus immobilization and would require little to no virus manipulation. While the small sample volume size $(10-20 \mathrm{~nL})$ of capillary electrophoresis is ideal for precise manipulations, CE-SELEX reduces the diversity of the library used in a selection by about ( $10^{5}$-fold reduction) even when very high library concentrations are used. An improved version of CE-SELEX termed Micro-Free Flow electrophoresis $(\mu \mathrm{FFE})$ compensates for a fraction of this reduction by increasing the total reaction volume ${ }^{68}$. $\mu \mathrm{FFE}$ adopts a continuous separation and collection of targets by streaming samples to a separation chamber where analytes are deflected laterally according to their electrophoretic mobility. 
M-SELEX was first established by the Soh group ${ }^{58}$ and utilizes paramagnetic beads, functionalized with the target protein and integrated into a microfluidic system. Several labs have used this approach after its first demonstration to generate aptamers with low nanomolar binding dissociation constants (in the range $10-50 \mathrm{nM}$ ) to different targets using fewer rounds (2-3 rounds) compared to traditional SELEX ( $\sim 10-20$ rounds). On the initial versions of this M-SELEX, several steps such as DNA amplification and strand separation, or in vitro RNA transcription were performed separately, thus extending the overall selection time. Recently, Olsen et al. reported a fully integrated version of this MSELEX that performed all steps of a SELEX round using the same device ${ }^{69}$. Using this innovative beads-based microfluidic technology, an IgE binding ssDNA aptamer (Kd, 12 $\mathrm{nM}$ ) was generated in 4 rounds in approximately 10 working hours. Another group reported complete automation of this platform to generate an enriched population of aptamers that target with low affinity (Kd 15nM) a lung cancer cell line (A549 cells) ${ }^{55}$. The entire process took about 3 days and included both negative and positive rounds of A549 targeting sequence enrichment. To put this in context, an average cell-SELEX takes about 3 weeks to obtain target enriched populations.

In the context of Virus-SELEX, a magnetic bead-based platform, such as M-SELEX, would require slight modification of the target virus particles to ensure their capture on magnetic beads. For example, our lab has developed a method to chemically biotinylate glycoproteins displayed on the surfaces of virus particles without disrupting virus infectivity (Chapters 4 and 5). However, conjugation of small chemical moieties such as biotin to virus glycoproteins can alter or reduce the availability of certain target epitopes during the aptamer selection. Thus, aptamers that bind these chemically modified virus 
proteins expressed on the surfaces of virus particles might not recognize or have the same affinity for the native, unmodified target glycoproteins.

\section{Bioinformatics guided aptamer screening.}

In recent years, aptamer selections have benefitted from the immense advancements in next-generation sequencing (NGS). This represents a significant upgrade from conventional shotgun cloning performed in early SELEX protocols on the final aptamer population, which was followed by their sequence identification through Sanger sequencing. Traditional screening methods rely on convergence and sequence frequency. Typically, sequences with the highest representation in the sequencing data are prioritized in the post-SELEX screening and characterization. However, work from our lab showed that underrepresented sequences could have the best properties. In that study, NGS analysis of an aptamer population with affinity to HIV-1 RT revealed clusters of sequences based on structural motifs. Further probing and comparisons within and among clusters identified a highly potent but underrepresented aptamer. The rare sequence $(0.4 \%$ of the enriched population) inhibited HIV-1 RT more potently than previously identified highly represented sequences ${ }^{70}$. NGS produces large data sets of candidate aptamer sequences that offers high-resolution coverage of the sequence representation in the final population. Within these data sets, some sequences will have a lower affinity for the target or display off-target binding. Such probes have to be excluded because they are not ideal for developing biosensors. Desired binding properties are defined by the final application of the aptamer. For example, virus-targeting aptamers should demonstrate high binding affinity to enable low limit of detection, and high specificity towards the 
target virus surface to reduce the number of false positives. Our lab and several others have developed bioinformatic tool kits to allow researchers to analyze these massive data sets. When combined with NGS, these tool kits can accelerate identification, characterization, and prioritization of aptamers that are suitable diagnostic probes. For example, FASTAptamer, an open-source bioinformatics toolkit developed in our lab can rank sequences according to their abundance in the analyzed pools, identify sequence clusters (conserved stretch motifs), and track enrichment of trajectories ${ }^{71}$. A recent report has introduced 'AptaSUITE' a novel toolkit that offers a more comprehensive data analysis, thus continuing to demonstrate the great number of innovations in bioinformatics guided aptamer development field ${ }^{72}$. Both toolkits allow researchers to analyze individual sequence evolution lineages for data-driven aptamer discovery. Post-SELEX screening and characterization of aptamer binding properties are timeconsuming and remain a bottleneck in the field. However, Soh's group developed a high throughput screening platform termed "Quantitative Parallel Aptamer Selection System" (QPASS $)^{73,74}$. Their platform can assess the affinity and specificity of about 104 aptamers in parallel. The QPASS platform hybridizes individual aptamer sequences onto a DNA microarray and incubates the samples with different concentrations of fluorescently labeled target molecules. The sequence affinities are deduced from the concentrationdependent signal output. Novel approaches that exploit a technology similar to the one used by QPASS could be easily integrated into a Virus-SELEX approach using either fluorescent protein-encoded virus surrogates (pseudotyped-viruses) or fluorescently labeled viral particles. 


\section{Aptamer-based diagnostic devices}

Aptamer-binding probes can be adapted to perform a number of functions, including as molecular recognition elements for the existing point-of-care (POC) device infrastructure. A variety of aptamer based POC devices have been reported, including devices or assays that combine aptamers and antibodies. A comprehensive review was published by Dhiman et al (citation). In this section, we highlight aptamer based POC assays such as sandwich detection methods and lateral flow tests, both of which are relatively low cost and simple to run.

Enzyme-linked oligonucleotide assays (ELONA) substitute antibodies with aptamers as probes in the classic ELISA. ELONA has been utilized to detect and quantify various analytes and pathogens including viruses. A comparison between ELISA and ELONA in detecting the bacterial pathogen Francisella tularensis showed ELONA to be slightly more sensitive than ELISA (LOD: ELONA= 1.7 x103 bacteria/ml, ELISA= 6.9 x103 bacteria/ml). ELONA can be developed with aptamer pairs or aptamers combined with antibodies. Different versions of ELONA have been demonstrated including the use of aptamers directly labeled with fluorescence probes or horseradish peroxidase (HRP) for signal amplification. Tseng et al. reported an integrated microfluidic sandwich assay that detects the H1N1 virus ${ }^{75}$. The system utilizes two aptamers; aptamer 1 mediates capture of virus and aptamer 2 is directly labeled with a fluorophore to provide a signal. In our lab, we have developed an antibody-aptamer sandwich assay that can detect MARV Glycoprotein (GP) displayed on a virus surrogate (rVSV/MARV GP). The platform utilizes anti-MARV sera as a capturing probe and a biotinylated MARV GP-specific aptamer as a molecular recognition/signal probe (chapter 4). 
Lateral flow assays (LFA) are well-established POC devices (pregnancy tests, glucose meters, insulin monitoring devices, flu tests) in the health care industry. Lateral flow devices (LFD) have a quick turnaround time (5-30 minutes), low cost, and are very easy to operate. They are excellent devices for diagnostics in resource-limited settings where POC tests are desperately needed for overall disease management. Some LFAs use nitrocellulose membranes (platform), gold nanoparticles (signal), and an affinity probe (antibodies or aptamers as alternatives) to produce qualitative and quantitative results. LFAs also require a pair of probes for capture and detection purposes. Currently, the majority of LFD utilize antibodies as probes. LFDs can be sandwich assay based or competitive assay based. In a sandwich format, the signal emanates from the targetmediated accumulation of probe-conjugated gold nanoparticles at a test line. Recently, Le et al. developed an H1N1 influenza-sensing LFD that exploit aptamers as affinity probes. The device utilizes a 5'-biotinylated RNA-aptamer (P30-10-16) as the affinity probe and an H1N1-antibody coated gold nanoparticle as the signal component. A similar device could be developed with two non-competing aptamers. However, the non-specific interaction between nucleic acids (especially DNAs) and nitrocellulose filters can negatively impact the signal-to-noise ratio. In addition, the affinity of aptamers to their targets might decrease upon conjugation to signal elements such as gold nanoparticles. Thus, during the aptamer screening, several tests need to be performed (e.g., binding assays before and after conjugation to gold NPs, assessment of background binding to nitrocellulose filters, etc.) to ultimately identify candidate aptamer sequences that are compatible with lateral flow devices. 


\section{Conclusion.}

Aptamers are excellent affinity probes for a wide range of targets including viruses. Over the past years, several technological advancements have improved the traditional SELEX protocol, making this approach more scalable and efficient in developing quality aptamers in record times. A fully integrated SELEX protocol that combines nucleobasemodified aptamers with enhanced chemical diversity, microfluidics, bioinformatics, and automation is needed to improve the aptamer discovery process (Fig 6.2). This improved selection platform might represent the natural evolution of the traditional SELEX process. The concrete possibility to develop virus-targeting aptamer in record times and use them as diagnostic probes might be an invaluable asset to use during novel virus disease outbreaks. In addition, as molecular recognition elements, virus-targeting aptamers can be implemented in the existing POC infrastructure with minimal changes, further accelerating the production of reliable and effective diagnostic devices. 


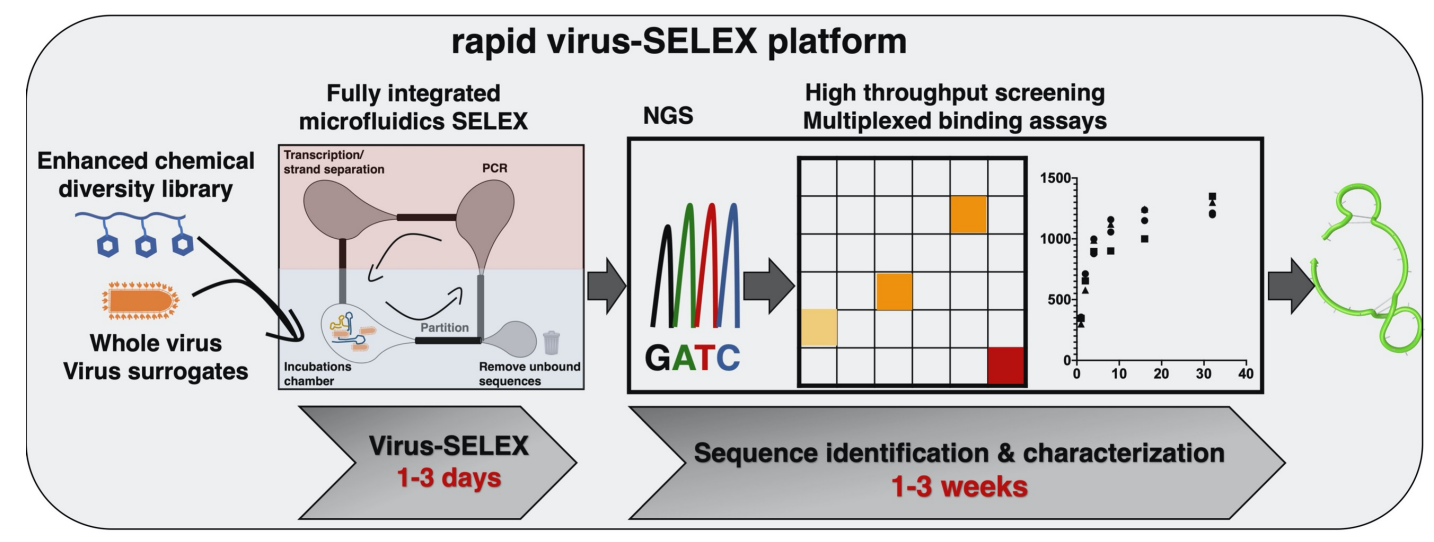

Figure 6.2. Overview of a rapid virus-SELEX platform. Such a platform would adopt a fully automated and integrated microfluidics SELEX platform that utilize enhanced chemical diversity nucleic acid library. A high throughput sequencing and screening process would help identify aptamer in short amount of time 


\section{Reference.}

1. Charlton, C. L. et al. Practical guidance for clinical microbiology laboratories: Viruses causing acute respiratory tract infections. Clinical Microbiology Reviews 32, (2019).

2. Green, S. M. et al. Clinical uncertainty, diagnostic accuracy, and outcomes in emergency department patients presenting with dyspnea. Arch. Intern. Med. 168, 741-748 (2008).

3. Hawthorn, A. et al. Peripheral Intravenous Catheter Protection : Br. J. Nurs. 30, 28 (2018).

4. Caliendo, A. M. et al. 28 Tobi Karchmer, 29 Ann T. MacIntyre, 30,31 L. Barth Reller, 32,33 and Audrey F. Jackson; 34; 25 Siemens Clinical Laboratory. Priv. Pract. Miami, Clin. Infect. Dis. 15, 139-70 (2013).

5. Jordan, B., Mitchell, C., Anderson, A., Farkas, N. \& Batrla, R. The Clinical and Health Economic Value of Clinical Laboratory Diagnostics. EJIFCC 26, 47-62 (2015).

6. Infectious Disease Emergence: Past, Present, and Future - Microbial Evolution and Co-Adaptation - NCBI Bookshelf. Available at:

https://www.ncbi.nlm.nih.gov/books/NBK45714/. (Accessed: 2nd April 2020)

7. Madhav, N. et al. Disease Control Priorities, Third Edition (Volume 9): Improving Health and Reducing Poverty. Disease Control Priorities, Third Edition (Volume 9): Improving Health and Reducing Poverty (The World Bank, 2017). doi:10.1596/978-1-4648-0527-1

8. Smith, K. F. et al. Global rise in human infectious disease outbreaks. J. R. Soc. 
Interface 11, 20140950 (2014).

9. Laboratory Diagnosis of Viral Infections. in Fenner's Veterinary Virology 105129 (Elsevier, 2017). doi:10.1016/B978-0-12-800946-8.00005-2

10. Storch, G. A. Diagnostic Virology. Clin. Infect. Dis. 31, 739-751 (2000).

11. Souf, S. Recent advances in diagnostic testing for viral infections. Biosci. Horizons Int. J. Student Res. 9, (2016).

12. Mehta, P. K., Raj, A., Pal Singh, N., Khuller, G. K. \& Promod Mehta, C. K. Detection of potential microbial antigens by immuno-PCR (PCR-amplified immunoassay). doi:10.1099/jmm.0.070318-0

13. Farid, S. S. Established bioprocesses for producing antibodies as a basis for future planning. Advances in Biochemical Engineering/Biotechnology 101, 1-42 (2006).

14. Morris, K. N., Jensen, K. B., Julin, C. M., Weil, M. \& Gold, L. High affinity ligands from in vitro selection: Complex targets. Proc. Natl. Acad. Sci. U. S. A. 95, 2902-2907 (1998).

15. Tuerk, C. \& Gold, L. Systematic evolution of ligands by exponential enrichment: RNA ligands to bacteriophage T4 DNA polymerase. Science (80-. ). 249, 505-510 (1990).

16. Ellington, A. D. \& Szostak, J. W. In Vitro Selection of RNA Molecules that Bind Specific Ligands. Nature 346, (1990).

17. Burke, D. H. \& Gold, L. RNA aptamers to the adenosine moiety of S-adenosyl methionine: structural inferences from variations on a theme and the reproducibility of SELEX. Nucleic Acids Research 25, (Oxford University Press, 1997). 
18. Burke, D. H., Scates, L., Andrews, K. \& Gold, L. Bent pseudoknots and novel RNA inhibitors of type 1 human immunodeficiency virus (HIV-1) reverse transcriptase. J. Mol. Biol. 264, 650-666 (1996).

19. Zhou, J. \& Rossi, J. Aptamers as targeted therapeutics: current potential and challenges. Nat Rev Drug Discov advance on, (2016).

20. Pan, W. et al. Isolation of virus-neutralizing RNAs from a large pool of random sequences. Proc. Natl. Acad. Sci. U. S. A. 92, 11509-11513 (1995).

21. Percze, K. et al. Aptamers for respiratory syncytial virus detection. Sci. Rep. 7, (2017).

22. Renders, M., Miller, E., Lam, C. H. \& Perrin, D. M. Whole cell-SELEX of aptamers with a tyrosine-like side chain against live bacteria. Org. Biomol. Chem. 15, 1980-1989 (2017).

23. Lünse, C. E. et al. An Aptamer Targeting the Apical-Loop Domain Modulates primiRNA Processing. Angew. Chemie Int. Ed. 49, 4674-4677 (2010).

24. Lee, J. F., Stovall, G. M. \& Ellington, A. D. Aptamer therapeutics advance. Current Opinion in Chemical Biology 10, 282-289 (2006).

25. Bruno, J. G. Predicting the uncertain future of aptamer-based diagnostics and therapeutics. Molecules 20, 6866-6887 (2015).

26. Zhou, J. \& Rossi, J. Aptamers as targeted therapeutics: Current potential and challenges. Nature Reviews Drug Discovery 16, 181-202 (2017).

27. Rogovik, A. L., Carleton, B., Solimano, A. \& Goldman, R. Palivizumab for the prevention of respiratory syncytial virus infection. Canadian Family Physician 56, 769-772 (2010). 
28. Mulangu, S. et al. A Randomized, Controlled Trial of Ebola Virus Disease Therapeutics. N. Engl. J. Med. 381, 2293-2303 (2019).

29. Vant-Hull, B., Payano-Baez, A., Davis, R. H. \& Gold, L. The mathematics of SELEX against complex targets. J. Mol. Biol. 278, 579-597 (1998).

30. Wang, J., Rudzinski, J. F., Gong, Q., Soh, H. T. \& Atzberger, P. J. Influence of Target Concentration and Background Binding on In Vitro Selection of Affinity Reagents. PLoS One 7, (2012).

31. Alam, K. K. et al. Poly-Target Selection Identifies Broad-Spectrum RNA Aptamers. Mol. Ther. - Nucleic Acids 13, 605-619 (2018).

32. Lai, H. C., Wang, C. H., Liou, T. M. \& Lee, G. Bin. Influenza A virus-specific aptamers screened by using an integrated microfluidic system. Lab Chip 14, 20022013 (2014).

33. Hicke, B. J. et al. Tenascin-C Aptamers Are Generated Using Tumor Cells and Purified Protein. J. Biol. Chem. 276, 48644-48654 (2001).

34. Liu, Y. et al. Aptamers selected against the unglycosylated EGFRvIII ectodomain and delivered intracellularly reduce membrane-bound EGFRvIII and induce apoptosis. Biol. Chem. 390, 137-144 (2009).

35. B Gopinath, S. C. et al. An RNA aptamer that distinguishes between closely related human influenza viruses and inhibits haemagglutinin-mediated membrane fusion. doi:10.1099/vir.0.81508-0

36. Klarkowski, D., O’Brien, D. P., Shanks, L. \& Singh, K. P. Causes of false-positive HIV rapid diagnostic test results. Expert Review of Anti-Infective Therapy 12, 4962 (2014). 
37. Lipman, N. S., Jackson, L. R., Trudel, L. J. \& Weis-Garcia, F. Monoclonal Versus Polyclonal Antibodies: Distinguishing Characteristics, Applications, and Information Resources. ILAR J. 46, 258-268 (2005).

38. Gold, L. et al. Aptamer-based multiplexed proteomic technology for biomarker discovery. PLoS One 5, e15004 (2010).

39. Renders, M., Miller, E., Lam, C. H. \& Perrin, D. M. Whole cell-SELEX of aptamers with a tyrosine-like side chain against live bacteria. Org. Biomol. Chem. 15, 1980-1989 (2017).

40. Wyatt, J. R. et al. Combinatorially selected guanosine-quartet structure is a potent inhibitor of human immunodeficiency virus envelope-mediated cell fusion. Proc. Natl. Acad. Sci. U. S. A. 91, 1356-1360 (1994).

41. Saccà, B., Lacroix, L. \& Mergny, J.-L. The effect of chemical modifications on the thermal stability of different G-quadruplex-forming oligonucleotides. Nucleic Acids Res. 33, 1182-92 (2005).

42. Odeh, F. et al. Aptamers chemistry: Chemical modifications and conjugation strategies. Molecules 25, (2020).

43. Pfeiffer, F., Rosenthal, M., Siegl, J., Ewers, J. \& Mayer, G. Customised nucleic acid libraries for enhanced aptamer selection and performance. Current Opinion in Biotechnology 48, 111-118 (2017).

44. Lapa, S. A., Chudinov, A. V. \& Timofeev, E. N. The Toolbox for Modified Aptamers. Molecular Biotechnology 58, 79-92 (2016).

45. Gawande, B. N. et al. Selection of DNA aptamers with two modified bases. Proc. Natl. Acad. Sci. U. S. A. 114, 2898-2903 (2017). 
46. Gold, L. et al. Aptamer-based multiplexed proteomic technology for biomarker discovery. PLoS One 5, (2010).

47. Tolle, F., Brändle, G. M., Matzner, D. \& Mayer, G. A Versatile Approach Towards Nucleobase-Modified Aptamers. Angew. Chemie Int. Ed. 54, 1097110974 (2015).

48. Laos, R., Thomson, J. M. \& Benner, S. A. DNA polymerases engineered by directed evolution to incorporate nonstandard nucleotides. Frontiers in Microbiology 5, 565 (2014).

49. Kimoto, M. \& Hirao, I. Site-specific incorporation of extra components into rna by transcription using unnatural base pair systems. Methods Mol. Biol. 634, 355-369 (2010).

50. Sefah, K. et al. In vitro selection with artificial expanded genetic information systems. Proc. Natl. Acad. Sci. U. S. A. 111, 1449-1454 (2014).

51. X-Ray crystal structure of SOMAmer reagent binding to its cognate protein. (2015).

52. Gawande, B. N. et al. Selection of DNA aptamers with two modified bases. doi:10.1073/pnas.1615475114

53. Kraemer, S. et al. From SOMAmer-based biomarker discovery to diagnostic and clinical applications: A SOMAmer-based, streamlined multiplex proteomic assay. PLoS One 6, (2011).

54. Rohloff, J. C. et al. Nucleic acid ligands with protein-like side chains: Modified aptamers and their use as diagnostic and therapeutic agents. Molecular Therapy Nucleic Acids 3, e201 (2014). 
55. Weng, C. H. et al. An automatic microfluidic system for rapid screening of cancer stem-like cell-specific aptamers. Microfluid. Nanofluidics 14, 753-765 (2013).

56. Millet, L. J. et al. Increasing access to microfluidics for studying fungi and other branched biological structures. Fungal Biol. Biotechnol. 6, 8 (2019).

57. Cho, M. et al. Quantitative selection of DNA aptamers through microfluidic selection and high-throughput sequencing. Proc. Natl. Acad. Sci. U. S. A. 107, 15373-15378 (2010).

58. Qian, J., Lou, X., Zhang, Y., Xiao, Y. \& Tom Soh, H. Generation of highly specific aptamers via micromagnetic selection. Anal. Chem. 81, 5490-5495 (2009).

59. Park, S. M. et al. Selection and elution of aptamers using nanoporous sol-gel arrays with integrated microheaters. Lab Chip 9, 1206-1212 (2009).

60. Ahn, J. Y., Jo, M., Dua, P., Lee, D. K. \& Kim, S. A sol-gel-based microfluidics system enhances the efficiency of RNA aptamer selection. Oligonucleotides 21, 93-100 (2011).

61. Dembowski, S. K. \& Bowser, M. T. Microfluidic methods for aptamer selection and characterization. Analyst 143, 21-32 (2018).

62. Eaton, R. M. et al. Selection of DNA aptamers for ovarian cancer biomarker HE4 using CE-SELEX and high-throughput sequencing. Anal. Bioanal. Chem. 407, 6965-73 (2015).

63. Mosing, R. K., Mendonsa, S. D. \& Bowser, M. T. Capillary electrophoresisSELEX selection of aptamers with affinity for HIV-1 reverse transcriptase. Anal. Chem. 77, 6107-6112(2005). 
64. Mendonsa, S. D. \& Bowser, M. T. In Vitro Evolution of Functional DNA Using Capillary Electrophoresis. J. Am. Chem. Soc. 126, 20-21 (2004).

65. Dong, L. et al. Screening and Identifying a Novel ssDNA Aptamer against Alphafetoprotein Using CE-SELEX. Sci. Rep. 5, 15552 (2015).

66. Jing, M. \& Bowser, M. T. Tracking the emergence of high affinity aptamers for rhVEGF165 during capillary electrophoresis-systematic evolution of ligands by exponential enrichment using high throughput sequencing. Anal. Chem. 85, 10761-10770 (2013).

67. Azizi, A. et al. Viral quantitative capillary electrophoresis for counting and quality control of RNA viruses. Anal. Chem. 84, 9585-9591 (2012).

68. Jing, M. \& Bowser, M. T. Isolation of DNA aptamers using micro free flow electrophoresis. Lab Chip 11, 3703-3709 (2011).

69. Olsen, T. et al. An integrated microfluidic SELEX approach using combined electrokinetic and hydrodynamic manipulation. SLAS Technol. 22, 63-72 (2017).

70. Ditzler, M. A. et al. High-throughput sequence analysis reveals structural diversity and improved potency among RNA inhibitors of HIV reverse transcriptase. Nucleic Acids Res. 41, 1873-1884 (2013).

71. Alam, K. K., Chang, J. L. \& Burke, D. H. FASTAptamer: A bioinformatic toolkit for high-throughput sequence analysis of combinatorial selections. Mol. Ther. Nucleic Acids 4, e230 (2015).

72. Hoinka, J., Backofen, R. \& Przytycka, T. M. AptaSUITE: A Full-Featured Bioinformatics Framework for the Comprehensive Analysis of Aptamers from HT-SELEX Experiments. Molecular Therapy - Nucleic Acids 11, 515-517 (2018). 
73. Cho, M. et al. Erratum: Quantitative selection of DNA aptamers through microfluidic selection and high-throughput sequencing (Proceedings of the National Academy of Sciences of the United States of America (2010) 107:35 (15373-15378) DOI: 10.1073/pnas.1009331107). Proceedings of the National Academy of Sciences of the United States of America 108, 5472 (2011).

74. Cho, M. et al. Quantitative selection and parallel characterization of aptamers. Proc. Natl. Acad. Sci. U. S. A. 110, 18460-18465 (2013).

75. Tseng, Y. T., Wang, C. H., Chang, C. P. \& Lee, G. Bin. Integrated microfluidic system for rapid detection of influenza H1N1 virus using a sandwich-based aptamer assay. Biosens. Bioelectron. 82, 105-111 (2016). 


\title{
Appendix 1: The effects of non-specific nucleic acid
}

\section{binding on the efficiency of complex target SELEX.}

\author{
(aka: Why "target purity" matters in a complex SELEX)
}

\section{Introduction}

SELEX has proven to be a robust and versatile tool for generating affinity probes (aptamers) since being described decades ago ${ }^{1,2}$. Aptamers can be developed for an

extensive spectrum of targets ranging from small molecules ${ }^{3}$, purified proteins ${ }^{4,5}$, and even viruses $^{6,7}$ bacteria $^{8}$, or mammalian cells ${ }^{9-12}$. SELEX performed to generate aptamers that have affinity for targets in complex contexts such as a cell surface can be challenging due to high levels of off-target binding ${ }^{13}$. Off target binding reduces the efficiency of the enrichment of target specific sequences in the library. Also, off-target binding could stem from the non-specific interaction between nucleic acids and the target protein or the partition platform used to isolate bound sequences. Complex targets such as viruses and whole cells tend to have high levels of off-target binding with nucleic acids as well if the target samples are not adequately purified ${ }^{14}$. This appendix describes a series of SELEX attempts that failed to generate filovirus surface targeting aptamers. I evaluate the impact of high background binding and high levels of off-target binding on the SELEX process. Finally, I highlight the experimental approaches that can be adopted to minimize background binding and off-target binding associated with partition platforms and complex targets respectively. 
The primary goal of the project was to develop nucleic acid aptamers that bind to filovirus surfaces. We utilized an ssDNA library with 56 nucleotides random region flanked by 20 nucleotide 5' and 3' primer binding sites. A short primer (anti-56N leader) that is complementary to the 5' primer binding site was annealed to the sequences in the library as described in chapter 4 . In all the selections, we used semi-purified virus particles (spun through 12\% iodixanol cushion) as targets. Retrospective EM analysis of the viral preparations (preps) purified in this manner showed protein and cellular debris contaminants in the samples suggesting the pelleting step alone was not enough for eliminating debris and protein contaminants (refer to chapter 3).

\section{Results and Discussion}

The first attempt to generate filovirus GP targeting aptamers involved two parallel selections against rVSV/MARV GP and rVSV/EBOV GP. A total of 8 rounds of positive selections were performed in each trajectory. We included two subtractive steps to deplete sequences that have nitrocellulose filters in rnds 4 and rnds 8 . The experimental details of the selection conditions are summarized in Table A1.1. After the 8th round, radiolabeled filter binding assay was used to evaluate the binding of the rnd 8 populations against their respective targets. The round 8 population from the MARV GP trajectory showed no improved binding over the starting library (Fig A1.1A). Both the starting library and the rnd 8 populations showed a weak dose-dependent signal when evaluated for binding to rVSV/MARV GP. The rnd 8 population from the EBOV trajectory showed a slightly stronger, but not significant dose-dependent signal compared to the starting library (Fig A1.1B). The rVSV/MARV GP trajectory was discontinued because 
the population showed no signs of target-specific binding. For the EBOV GP trajectory, we performed four additional rounds with increasing stringency (reduced target concentration) in efforts to improve the binding signal. Binding assessment of the rnd 12 population on rVSV/MARV_GP virus showed weak dose-dependent binding signal, compared to the starting library (Fig A1.1C). The results suggested that both selections were not able to generate a target-enriched population that is deserving of further enrichment or characterizing. 

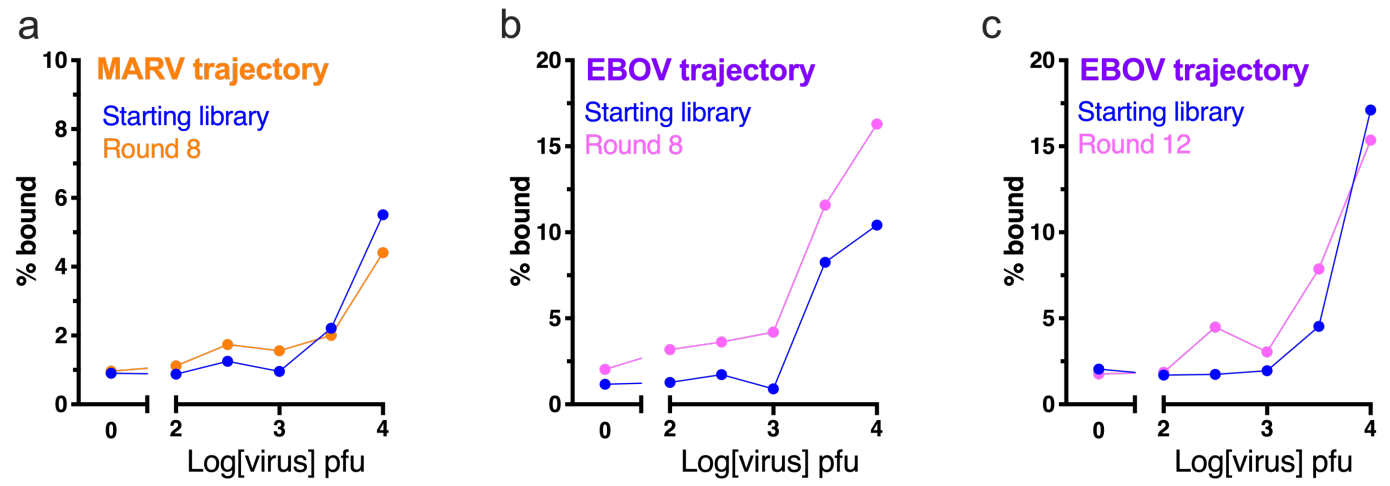

Figure A7.1. Biding specificity of ssDNA libraries selected for binding to MARV GP and EBOV GP. For the MARV trajectory, three radiolabeled ssDNA inputs (starting library blue and MARV-round 8 orange, and EBOV-round 8 purple) were evaluated for dose-dependent binding to their respective targets. A. Both the starting library and the round 8 population from the MARV trajectory show low level, weak dosedependent signal against rVSV/MARV GP. B. Rnd 8 population from the EBOV trajectory showed a slightly better dose-dependent signal over the starting library when assessed against rVSV/EBOV GP. C. The rnd 12 population from the MARV trajectory shows a similar, weak dose-dependent signal as the starting library when assessed against the rVSV/MARV GP.

3. 
Table A7.1. Summary of selection conditions for filovirus SELEX attempt 1

\begin{tabular}{c|ccccc}
$\begin{array}{c}\text { Selection } \\
\text { round }\end{array}$ & [ssDNA] & $\begin{array}{c}\text { Volume } \\
(\boldsymbol{\mu} \mathbf{L})\end{array}$ & $\begin{array}{c}\text { Amount of virus } \\
(\mathbf{p f u})\end{array}$ & $\begin{array}{c}\text { Subtraction } \\
\text { step }\end{array}$ & $\begin{array}{c}\text { Washing step. } \\
(\mathbf{0 . 5 m L} \text { binding buffer) }\end{array}$ \\
\hline Round 1 & 1 & 500 & $10^{4}$ & & $2 \mathrm{X}$ \\
Round 2 & 1 & 500 & $10^{4}$ & & $2 \mathrm{X}$ \\
Round 3 & 1 & 500 & $10^{4}$ & nitrocellulose & $2 \mathrm{X}$ \\
Round 4 & 1 & 500 & $10^{4}$ & & $2 \mathrm{X}$ \\
Round 5 & 1 & 300 & $10^{4}$ & & $2 \mathrm{X}$ \\
Round 6 & 1 & 300 & $10^{4}$ & $2 \mathrm{X}$ \\
Round 7 & 1 & 300 & $10^{4}$ & & $2 \mathrm{X}$ \\
Round 8 & 1 & 300 & $10^{4}$ & nitrocellulose & $2 \mathrm{X}$ \\
Round 9 & 1 & 300 & $10^{3}$ & & $2 \mathrm{X}$ \\
Round 10 & 1 & 300 & $10^{3}$ & & $2 \mathrm{X}$ \\
Round 11 & 1 & 300 & $10^{3}$ & & $2 \mathrm{X}$ \\
Round 12 & 1 & 300 & $10^{3}$ & nitrocellulose &
\end{tabular}

Background binding and off-target binding have significant impacts on the outcome of a selection. Reported experimental works and mathematical models on SELEX show that an increase in background binding decreases the maximum attainable target specific. enrichment exponentially. Besides, large amounts of the target are required for targetspecific enrichment when background and off-target binding are high ${ }^{15,16}$. In every round in the previous selection attempt, nitrocellulose filters used for partitioning were soaked in potassium hydroxide $(\mathrm{KOH})$ to reduce non-specific interaction with the ssDNA library. We identified two potential causes for the failed SELEX attempt. First, crude virus preps presented high levels of off-target binding. Second, the nitrocellulose filters still had high background binding, despite the $\mathrm{KOH}$ treatment. 
To investigate the second speculation, we assessed the binding of the round 12 populations in the presence of salmon sperm DNA as non-specific binding competitors of the ssDNA library. The filter retention assay showed a about $50 \%$ decrease in the binding signal from both the starting library and the round 12 population (Fig A1.2). This suggested that a significant portion of the binding events in every round resulted from non-specific interactions. We reasoned that the addition of non-specific binding competitors could lower background binding. A more stringent washing step could also help deplete non-specific binders. Both of these potential solutions were implemented in subsequent selection attempts, including the successful attempt described in chapters 4 and 5 .

Finally, the high non-specific background also suggested that high target concentrations of virus might be needed to promote target-specific enrichment. We deduced that possibly, an inadequate number of targets present in the opening rounds of selections resulted in a higher stringency. A high stringency at such an early stage could bottleneck the selection by eliminating promising sequences. Moving forward, we elected to increase the number of virus particles to be used for the early rounds of selection. 


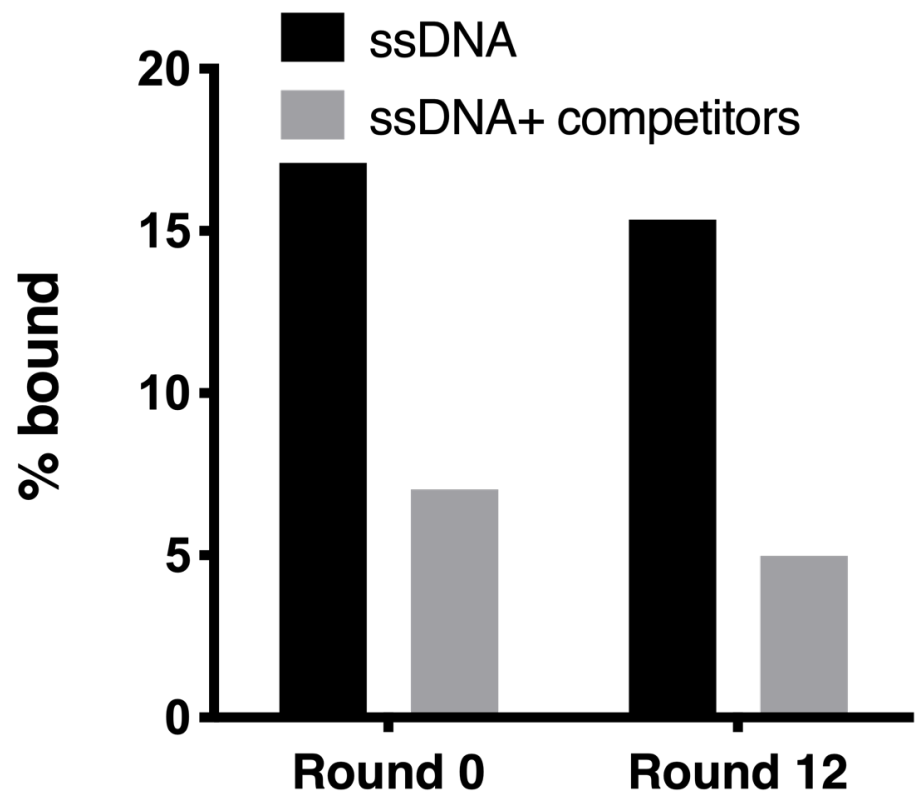

Figure A 7.2. Filter binding assay assessment shows salmon sperm DNA reduces the background binding of nucleic acids. Radioactive filter binding assessment of round 12 population was performed in the presence (gray) and absence (black) of competitors. 
Second selection attempt identified unknown target enriched sequences.

The second attempt focused on single trajectory selection using the same library (5' pbs sequestered) against rVSV/MARV GP. However, some conditions were modified to minimize background binding, which reflecting the lessons learned from the previous selection attempt. First, the concentration of the rVSV/MARV GP was increased from $10^{4}$ to $10^{5} \mathrm{pfu}$ for the opening rounds. Salmon sperm DNA $(0.2 \mu \mathrm{g} / \mathrm{ml})$ was included in the incubation as non-specific competitors in the fourth round onwards. Finally, we increased the number of washing steps performed at every round from a single $500 \mu \mathrm{L}$ wash to $1-2 \mathrm{ml}$ washes. Overall, 12 rounds of positive selection were performed, including 3 subtractive steps performed to deplete sequences that have affinity for VSV core. The subtractive steps were done at rnds 8.1, 10.1, and 12.1. Table A1.2 shows a summary of the selection conditions applied in each round.

Following the 12th round, we performed filter retention assays to evaluate rnds 4.1, 8.1, and 12.1 populations. The rnd 4.1 population showed insignificantly better binding to the rVSV/MARV_GP compared to the starting library (Fig A1.3A). Both rounds-8.1 and 12.1 populations showed a strong dose-dependent signal compared to the starting library (Fig A1.3B and 3C). Rnd 12.1 population showed a steeper curve compared to that of round 8 (Fig A1.3C). Together these results suggested an enrichment of rVSV/MARV_GP binding sequences after several rounds of selection. Next, we evaluated the binding specificity of the enriched populations by assessing the binding of both populations on rVSV/EBOV and VSV. We observed a strong dose-dependent signal to VSV-EBOV, suggesting pan-filovirus glycoprotein recognition (Fig A1.3B and A1.3C). In contrast, the 
populations from both rounds showed a weaker viral dose-dependent signal towards VSV, suggesting some form of specificity toward filoviruses (Fig A1.3C). 
4.

Table A1 7.2. Summary of selection conditions f filovirus SELEX attempt 1

\begin{tabular}{|c|c|c|c|c|c|c|}
\hline & $\begin{array}{c}\text { [ssDNA] } \\
(\mu \mathrm{M})\end{array}$ & $\begin{array}{c}\text { Competitors } \\
(\mathrm{mg} / \mathrm{ml})\end{array}$ & Volume & $\begin{array}{c}\text { Amount } \\
\text { of virus } \\
\text { (pfu) }\end{array}$ & $\begin{array}{l}\text { Subtraction } \\
\text { step }\end{array}$ & $\begin{array}{c}\text { Washing } \\
\text { step. } \\
\text { (1mL } \\
\text { binding } \\
\text { buffer) }\end{array}$ \\
\hline Round 1.1 & 1 & - & 500 & $10^{5}$ & & $1 X$ \\
\hline Round 2.1 & 1 & - & 250 & $10^{5}$ & & $1 X$ \\
\hline Round 3.1 & 1 & - & 250 & $10^{5}$ & & $1 X$ \\
\hline Round 4.1 & 1 & 0.2 & 250 & $10^{5}$ & nitrocellulose & $2 X$ \\
\hline Round 5.1 & 1 & 0.2 & 250 & $10^{5}$ & & $2 X$ \\
\hline Round 6.1 & 1 & 0.2 & 250 & $10^{5}$ & & $2 X$ \\
\hline Round 7.1 & 1 & 0.2 & 250 & $10^{5}$ & & $2 X$ \\
\hline Round 8.1 & 1 & 0.2 & 250 & $10^{5}$ & VSV & $2 \mathrm{X}$ \\
\hline Round 9.1 & 1 & 0.2 & 250 & $10^{5}$ & & $2 X$ \\
\hline Round 10.1 & 1 & 0.2 & 250 & $10^{5}$ & VSV & $2 X$ \\
\hline Round 11.1 & 1 & 0.2 & 250 & $10^{5}$ & & $2 X$ \\
\hline Round 12.1 & 1 & 0.2 & 250 & $10^{5}$ & VSV & $2 X$ \\
\hline
\end{tabular}



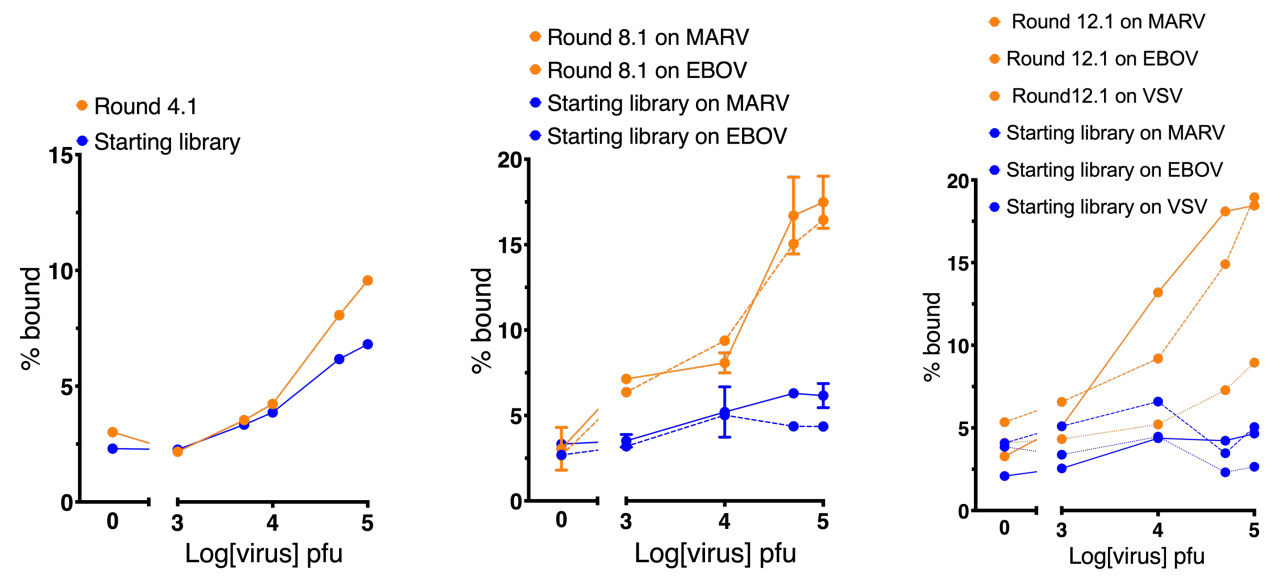

Figure A 7.3. Biding and binding specificity of ssDNA libraries selected for binding to MARV GP. Four radiolabeled ssDNA inputs groups (starting library blue and rounds $(4.1,8.1$, and 12.1$)$ orange) were evaluated for dosedependent binding to their respective targets and non-targets. A. Round 4.1 population shows a slightly stronger dose-dependent signal over the startinglibrary when assessed against rVSV/MARV GP. B. Round 8.1 population shows a strong dose-dependent binding signal over the starting library when assessed against rVSV/MARV GP (target) and rVSV/EBOV GP (non-target filovirus). C. Rnd12.1 population shows a strong dose-dependent signal over the starting library when assessed against rVSV/MARV GP and rVSV/EBOV GP. Both library inputs show a low level, weak dose-dependent binding signal when assessed against VSV (non-target and non-filovirus). 
The results were encouraging but concerning due to the lack of specificity for the enriched population. Although MARV GP and EBOV GP share common structural features, the primary amino acid sequence of the two GPs share only $\sim 30 \%$ identity $^{17}$. Moreover, pan-filovirus GP antibodies are rare ${ }^{18}$. Despite these concerns, we proceeded to the next steps with the enriched library for several reasons. (1). Aptamers are relatively small compared to antibodies; therefore, they can access surfaces and epitopes that are otherwise inaccessible to larger molecules. (2). Filovirus glycoproteins are heavily glycosylated, and the sequences could be targeting common filovirus-specific glycosylation patterns. (3). Some of the individual sequences from the enriched population could be strain specific.

We subjected the enriched populations to low throughput sequencing and analysis to get the initial sense of sequence representation in both populations. Both rnds 8.1 and 12.1 were cloned into plasmid vectors, transformed into bacteria, and isolate plasmids from individual colonies. We obtained 18 sequences from rnds 8.1 and 15 sequences from rnds 12.1. Sequence analysis identified four clusters of sequences for the round-8.1 population (Table A1.3). Cluster-1 represented a sequence that was sampled four times out of 18 total sequences. The remaining two clusters each had a sequence that was sampled twice out of the 18 identified. Cluster-4 had 10 sequences that were all unique (Table A1. 4).

Initial filter binding assay-based screen for sequences that bind to rVSV/MARV GP identified some good binders (>3-fold binding over background) (Fig A1.4A). However, all the sequences that showed strong binding signal for rVSV/EBOV_GP, showed same 
signal when assessed on rVSV/MARV_GP. This suggested a potential pan-filovirus GP binding property. Alternatively, the sequences could be targeting a common contaminant in the virus samples. We increasingly favored the latter interpretation after EM analysis of the virus preps showed significant levels of protein contaminants and cellular debris. We generated relatively pure viral particles using a three-step density gradient purification process (refer to chapter 3 ). We then evaluated binding of the enriched populations (rnd 8.1 and $\mathrm{rnd} 12.1$ ) to the new virus preps. Both populations failed to bind to the new virus preps. The result suggest that the enriched sequences may be binding to an unknown molecule in the old virus prep. 


\section{Table A 7.3. Sanger sequencing data from rnd 8.1 population.}

Clone

Cluster

\begin{tabular}{|c|c|}
\hline & Cluster 1 \\
\hline$M A R V \_8.1 \_C 5$ & ACCAACATGTTGTCCGGTAGGTCGTCACCCCATACCGATGCGTCTAAGGGGAGCAA \\
\hline$M A R V_{-} 8.1-C 6$ & ACCAACATGTTGTCCGGTAGGTCGTCACCCCATACCGATGCGTCTAAGGGGAGCAA \\
\hline$M A R V_{-} 8.1 \_C 10$ & ACCAACATGTTGTCCGGTAGGTCGTCACCCCATACCGATGCGTCTAAGGGGAGCAA \\
\hline$M A R V_{-} 8.1 \_C 19$ & ACCAACATGTTGTCCGGTAGGTCGTCACCCCATACCGATGCGTCTAAGGGGAGCAA \\
\hline & Cluster 2 \\
\hline $\begin{array}{l}\text { MARV_8.1 }-\mathrm{C} 2 \\
\text { MARV_8.1 _C13 }\end{array}$ & $\begin{array}{l}\text { CTGAATGGTCCCGGGTCTCGCAGTCATCCGGTGTTTACTTAGAAATCAACCCAGTA } \\
\text { CTGAATGGTCCCGGGTCTCGCAGTCATCCGGTGTTTACTTAGAAATCAACCCAGTA }\end{array}$ \\
\hline & Cluster 3 \\
\hline $\begin{array}{l}\text { MARV_8.1 }-C 9 \\
\text { MARV_8.1 _C16 }\end{array}$ & $\begin{array}{l}\text { ССTATGAGATCCCGAACGGTGCATAAGAGGCATGGTGTGAGGTTTACTTTCCCGAT } \\
\text { ССТATGAGATCCCGAACGGTGCATAAGAGGCATGGTGTGAGGTTTACTTTCCCGAT }\end{array}$ \\
\hline & Unique sequences \\
\hline MARV_8.1_C20 & САTCCCCCTGGTTCGCTGGGAGCATATGCTCACATTGCCTTGCCTTATTTGGGGGA \\
\hline MARV_8.1_C7 & TGCAATATGTTGTC GGCAATAGTCATCATTATCCATACAAATCGCCGATAGGCCGA \\
\hline MARV_8.1_C12 & CСАATATGCTGTCACGGAGGTCATCAATGCCCTAAAAAAGGATCAAACTTTCСTTT \\
\hline MARV_8.1_C1 & ATGACAAAATCGGGGTAGTCAACACACCTTAAAATCGCAGAACAGTTTCAACATAA \\
\hline MARV_8.1_C3 & TTAAGCAAAAGATGTTGAGGGTGGGTCAGATGTCGCTCATTAGTTTCAAAAGAATA \\
\hline MARV_8.1_C4 & TATGCCACTAATGTGTAATGGACACATGTTGTTCACGGACCCGCTTGGTAGCATTT \\
\hline MARV_8.1_C8 & TACCAAACAGTATGTGCAAACTACCCGAGTTTACGTTTTCAGCTTCGCCGTTCCTC \\
\hline MARV_8.1_C11 & ССТАTTCTACGCAATAGTCAGGAACCCGTCTCTCGGTCAACAGCAGATTCAAАAAT \\
\hline MARV_8.1_C17 & САCTGCTGCCTAGCCCCATAGTATCCGCACCACGCCAGACTCATATGTTAAACAGT \\
\hline MARV_8.1_C15 & CATAATAGCCCCAGCGGCGTCACATATGTGTCATGAGCAGTCGTAATTCCCGTAGA \\
\hline
\end{tabular}


5.

Table A 7.4. Sanger sequencing data from rnd 12.1 population.

\begin{tabular}{|c|c|}
\hline Clone & Cluster \\
\hline & Cluster 1 \\
\hline MARV12.1C9 & CAGGTCTCGAGAGTCTGAACGCACTTCTCACACССТCCTACAAAGGCTACTCCAAА \\
\hline MARV12.1C17 & CAGGTCTCGAGAGTCTGAACGCACTTCTCACACCСTCCTACAAAGGCTACTCCAАA \\
\hline & Unique sequences \\
\hline MARV12.1C1 & GCCCAAACGTCTGGCTAAGTCGCAGGATTCATCCTGATCCTTCACATCTCCGCACA \\
\hline MARV12.1C3 & ATGTGTCCACAGATAGGCCCTCGCCGAGCATGATACAAAAGTGCGTGAGAGTTAAT \\
\hline MARV12.1C4 & GCACTAGATGACACTCGGGGGAGATGAATTAATATGTGATAAAGATTAATGAATTC \\
\hline MARV12.1C5 & GAGAGTTAGGGCTGGAATTGTAATGTGAACACTGCAGATGATTTCAAACTATGTGC \\
\hline MARV12.1C6 & TCGACCCCCGTCTAGGTACCGACCAACACATGACCAGCATGGGATAACCATGCAAA \\
\hline MARV12.1C7 & TACCAAACAGTATGTGCAAACTACCCGAGTTTACGTTTTCAGCTTCGCCGTTCCTC \\
\hline MARV12.1C8 & ATGGCATCGCAAGGTAACTGAAATGGAATCGGCAAAGATTAGGAATCGAGTAGTGG \\
\hline MARV12.1C10 & ATGGTAACCTACCCATGCTCACGTAGCTACAATGGTAGAGCAGCTTGACTTGATAT \\
\hline MARV12.1C13 & GTGAATGATGCGATCTCTGCCAАCCACСАCTCACAGAAGACTCCACTGTTACCAАT \\
\hline MARV12.1C14 & TTTGTCCCCACATAGGTCTCGCGAAGCATTTACCACATGAGTGCATATCATGACCT \\
\hline MARV12.1C15 & GTAACGTCCAACCTGATTGATCATGTTACTCCTAATCGTAACACTTGACTTTATAT \\
\hline MARV12.1C16 & TGCAATATGTTGTCGGCAATAGTCATCATTATCCATACAAATCGCCGATAGGCCGA \\
\hline MARV12.1C19 & GGAACACTGGAATAACACTAGAGCGAGATGTACAGATATTCAACATGCATAGGTTG \\
\hline
\end{tabular}




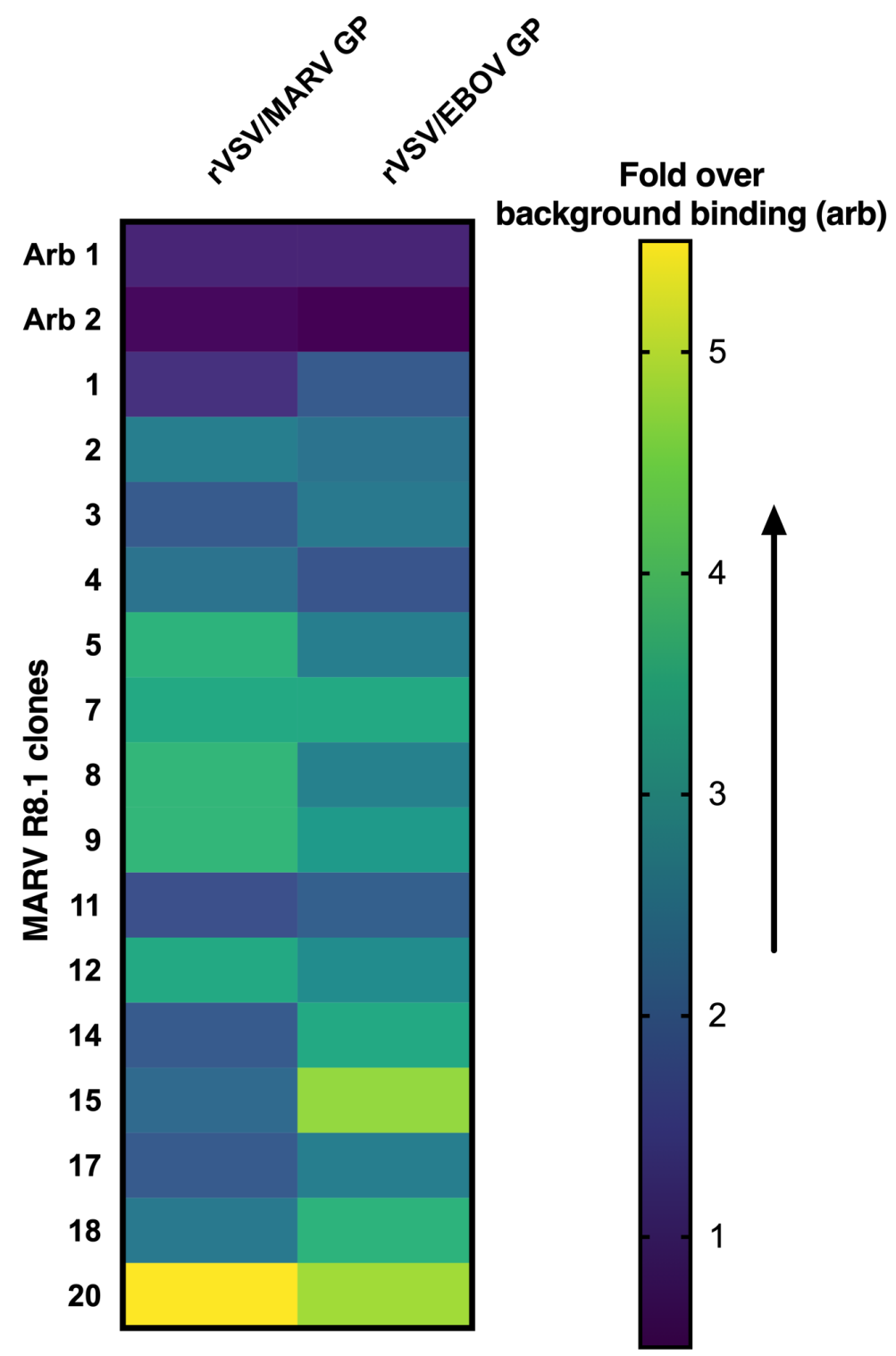

Figure A7.4. A heat map representation for binding assess of individual sequences against rVSV/MARV GP (target) and rVSV/EBOV GP (non-target filovirus GP). Two arbitrary ssDNA sequences (arb $1 \&$ arb) were used as non-binding controls (background binding). 


\section{Conclusion.}

High background and off-target binding can impair target-specific enrichment during aptamer selections. SELEX against complex targets such as virus particles can be challenging due to the high level of off-target binding. Also, low-efficiency partitioning platforms such as nitrocellulose filters are associated with high levels of background binding. Here, we described strategies that promote target-specific enrichment when performing selections against complex targets such as virus particles. We showed that for low efficiency partition platforms such a nitrocellulose filters, relatively pure virus particle preps are required to facilitate target-specific enrichment. Also, the inclusion of non-specific binging competitors can mitigate the background binding of the library to the target and partition platform. We observed that subtractive steps alone are not sufficient to eliminate weaker and non-binding sequences. While the target concentration can be reduced to facilitate competition, it is crucial to have an optimal number of virus particles during the initial rounds of selection to reduce the risk of losing promising sequences. All these strategies were applied in the virus selections described in chapters 4 and 5. 


\section{References}

1. Tuerk, C. \& Gold, L. Systematic evolution of ligands by exponential enrichment: RNA ligands to bacteriophage T4 DNA polymerase. Science (80-. ). 249, 505-510 (1990).

2. Ellington, A. D. \& Szostak, J. W. In vitro selection of RNA molecules that bind specific ligands. Nature 346, 818-822 (1990).

3. Burke, D. H. \& Gold, L. RNA aptamers to the adenosine moiety of S-adenosyl methionine: structural inferences from variations on a theme and the reproducibility of SELEX. Nucleic Acids Research 25, (Oxford University Press, 1997).

4. Burke, D. H., Scates, L., Andrews, K. \& Gold, L. Bent pseudoknots and novel RNA inhibitors of type 1 human immunodeficiency virus (HIV-1) reverse transcriptase. J. Mol. Biol. 264, 650-666 (1996).

5. Dausse, E., Da Rocha Gomes, S. \& Toulmé, J. J. Aptamers: a new class of oligonucleotides in the drug discovery pipeline? Current Opinion in Pharmacology 9, 602-607 (2009).

6. Pan, W. et al. Isolation of virus-neutralizing RNAs from a large pool of random sequences. Proc. Natl. Acad. Sci. U. S. A. 92, 11509-11513 (1995).

7. Percze, K. et al. Aptamers for respiratory syncytial virus detection. Sci. Rep. 7, (2017).

8. Chen, F., Zhou, J., Luo, F., Mohammed, A. B. \& Zhang, X. L. Aptamer from 
whole-bacterium SELEX as new therapeutic reagent against virulent Mycobacterium tuberculosis. Biochem. Biophys. Res. Commun. 357, 743-748 (2007).

9. $\quad$ Morris, K. N., Jensen, K. B., Julin, C. M., Weil, M. \& Gold, L. High affinity ligands from in vitro selection: Complex targets. Proc. Natl. Acad. Sci. U. S. A. 95, 2902-2907 (1998).

10. Cerchia, L., Esposito, C. L., Jacobs, A. H., Tavitian, B. \& de Franciscis, V. Differential SELEX in Human Glioma Cell Lines. PLoS One 4, e7971 (2009).

11. Hicke, B. J. et al. Tenascin-C Aptamers Are Generated Using Tumor Cells and Purified Protein. J. Biol. Chem. 276, 48644-48654 (2001).

12. Zhou, J. \& Rossi, J. Aptamers as targeted therapeutics: current potential and challenges. Nat Rev Drug Discov advance on, (2016).

13. Raddatz, M. L. et al. Enrichment of Cell-Targeting and Population-Specific Aptamers by Fluorescence-Activated Cell Sorting. Angew. Chemie Int. Ed. 47, $5190-5193(2008)$.

14. Shamah, S. M., Healy, J. M. \& Cload, S. T. Complex Target SELEX. (2007). doi:10.1021/ar700142z

15. Wang, J., Rudzinski, J. F., Gong, Q., Soh, H. T. \& Atzberger, P. J. Influence of Target Concentration and Background Binding on In Vitro Selection of Affinity Reagents. PLoS One 7, (2012).

16. Ozer, A., White, B. S., Lis, J. T. \& Shalloway, D. Density-dependent cooperative non-specific binding in solid-phase SELEX affinity selection. doi:10.1093/nar/gkt477 
17. Brindley, M. A. et al. Ebola Virus Glycoprotein 1: Identification of Residues Important for Binding and Postbinding Events. J. Virol. 81, 7702-7709 (2007).

18. Holtsberg, F. W. et al. Pan-ebolavirus and Pan-filovirus Mouse Monoclonal Antibodies: Protection against Ebola and Sudan Viruses. J. Virol. 90, 266-278 (2016). 


\section{Vita}

Kwaku Dwumah Tawiah was born in Seikwa, Ghana, on November 15, 1989. He attended Mim Model Basic School and continued to high school at St Augustine's College in Cape Coast, Ghana. Kwaku relocated to the United States in 2009 to attend college and in 2012, received the degree of Bachelor of Science in Chemistry with an emphasis in Biochemistry from Lindenwood University. He moved on to work at Monsanto, American Bottoms Water Treatment Facility and BioMèrieux Inc as a lab technician. In 2014, Kwaku matriculated in the doctoral program of the University of Missouri's Department of Biochemistry 